Historic, archived document

Do not assume content reflects current scientific knowledge, policies, or practices. 

Forest Service

Northeastern Forest

Experiment Station

Resource Bulletin NE-110



Robert T. Brooks

Dawn M. DiGiovanni

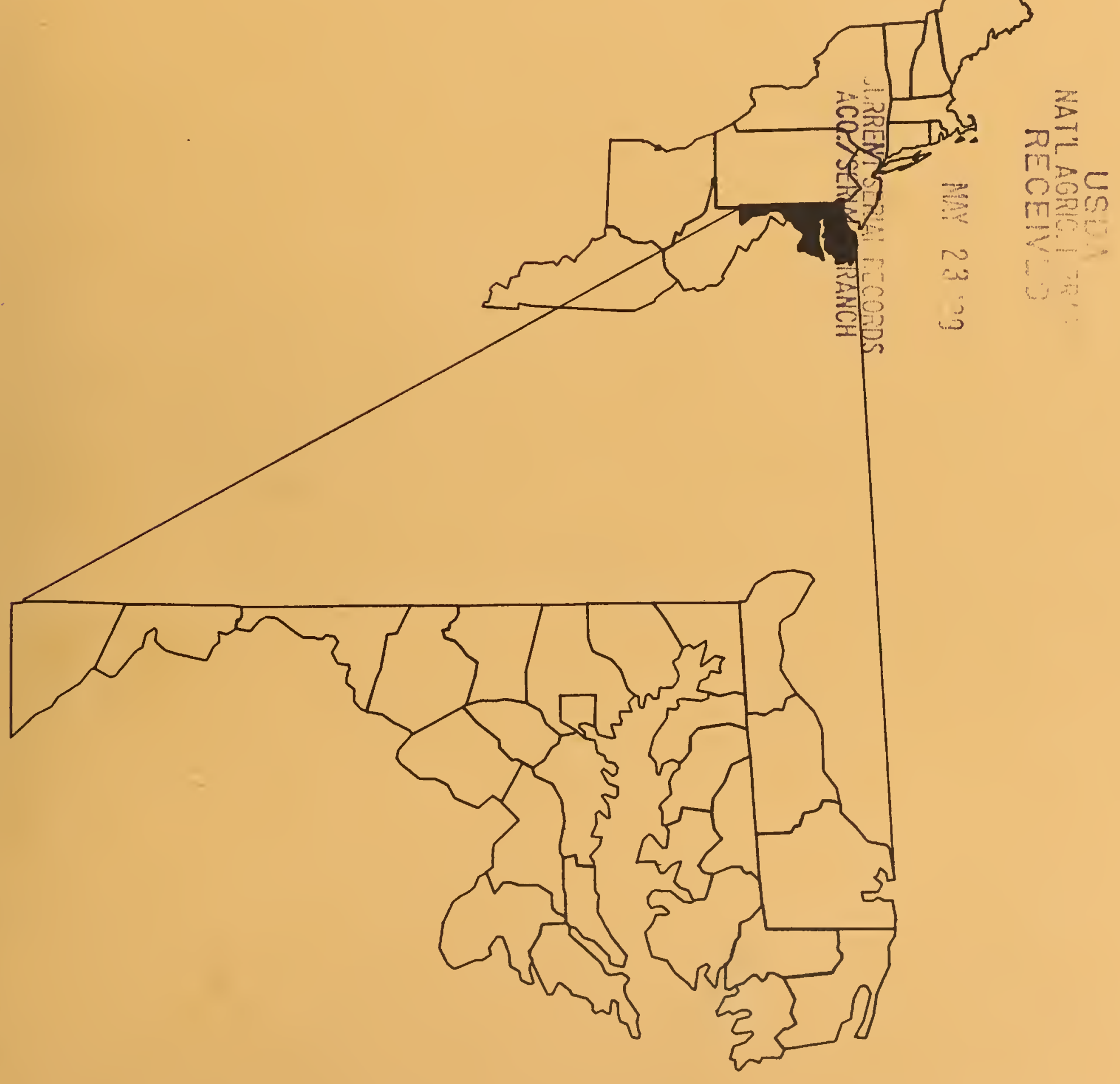




\section{Abstract}

This is a statistical report on the first forest wildlife habitat survey of Maryland and Delaware conducted in 1985-86 by the Forest Inventory and Analysis Unit, Northeastern Forest Experiment Station, U.S. Department of Agriculture, Broomall, Pennsylvania. Results are displayed in 118 tables covering forest area, ownership, land pattern, mast potential, standing dead and cavity trees, and understory woody-stemmed vegetation. Data are presented at county and/or unit and state levels of resolution.

\section{Foreword}

The reinventories of Maryland and Delaware are under the overall direction of John R. Peters, Project Leader of the Forest Inventory and Analysis Unit. Thomas W. Birch assisted in the development and administration of the operating plan. Charles T. Scott was responsible for the design of the inventory and sample selection. David J. Alerich supervised the interpretation of aerial photos and collection of data. He was assisted by Joseph $G$. Reddan. Members of the field staff were:

Lisa M. Arbucci
Theresa Arre
Vanessa Artman-Bailey
Michael J. Barton, Jr.
Barron E. Burch, III
Stephen M. Clark
Dawn M. DiGiovanni
Thomas V. Gabriel
David W. Guest
Roger L. Huber
Robert E. Ilgenfritz
Lawrence A. Ingerson
Judith T. Keyes
Ann K. Kessler
Wayne R. Kettlewood
Melanie Leavitt

Michael L.Longo
Frank Lopez
Anna M. Massey
Richard A. McCullough
Worthen D. Muzzey, Jr.
Wayne Noll
Glen A. Onusseit
Stephen C. Parrett
Jennifer L. Quinn
Joyce P. Sawicki
James E. Semp
Peter Smith
Jonathan W. Spencer
Tina J. Terrell
Robert Wells
Teresa L. Witzel

Thomas S. Frieswyk and Dawn M. DiGiovanni applied FINSYS (Forest INventory SYStem), a generalized data processing system, to the specific needs of the Maryland inventory and produced summary tables for the state and counties. Thomas W. Birch and Dawn M. DiGiovanni were instrumental in assuring that the area estimates were consistent with the two previous inventories. Rosemary K. Venit produced the graphics and was involved in rewriting parts of the FINSYS table generating routine.

Marie Pennestri was responsible for administrative and secretarial services. Dorelle Smith typed the text for this report.

The Forest Inventory and Analysis Unit would like to thank the landowners of Maryland and Delaware for their cooperation and assistance during this inventory. 


\title{
Forest Wildlife Habitat Statistics for Maryland and Delaware--1986
}

\author{
The Authors \\ Robert T. Brooks, Research Wildlife Biologist, \\ Forest Inventory and Analysis Unit, \\ Northeastern Forest Experiment Station, \\ USDA Forest Service, Broomall, PA. \\ Dawn M. DiGiovanni, Forester, Forest Inventory \\ and Analysis Unit, Northeastern Forest \\ Experiment Station, USDA Forest Service, \\ Broomall, PA.
}




\section{Contents}

$\begin{array}{lr}\text { Highlights } & 1 \\ \text { Introduction } & 2 \\ \text { Reliability of the Estimates } & 2 \\ \text { Definition of Terms } & 3 \\ \text { References } & 8\end{array}$

Trees, Shrubs, and Vines of the
Northeast

Ecological Importance and Relative

Distribution of Lesser Woody-

Stemmed Species, Maryland and

Delaware, 1986

Ecological Importance and Relative

Distribution of Trees $5.0+$ d.b.h.

by Species, Maryland and

Delaware, 1986

Metric Equivalents

Index to Tables

Maryland

State

27

Central Unit

Lower Eastern Shore Unit

Southern Unit

Western Unit

28

County

Delaware

State

County 


\section{Highlights}

* Maryland, with 2.7 million acres of forest land, is 43 percent forested. Delaware, with nearly 400,000 acres of forest land, is 32 percent forested.

* Ninety percent or more of each state's forest land is classified as timberland, formerly termed commercial forest land.

* Each state has experienced a slight decrease in timberland area since its previous forest survey.

* In Maryland, the Central Unit is the least heavily forested, when timberland is expressed as a percentage of total land area.

* The timberland area of Kent and New Castle Counties, Delaware, when expressed as a percentage of total land area, is comparable to that of Maryland's Central Unit, and Sussex County, Delaware, is comparable to Maryland's Lower Eastern Shore Unit.

* Landscape patterns are least diverse in Maryland's Lower Eastern Shore and most diverse in Central Maryland's Baltimore-Washington corridor.

* Timberland is predominately a privately owned resource.

* The area of sawtimber stands has increased since the surveys of the 1970's while the area of seedling and sapling stands has declined to only 10 percent (Maryland) to 18 percent (Delaware) of timberland area.

* Oak/hickory is the dominant forest type group, occupying 42 percent (Delaware) to 60 percent (Maryland) of timberland area.

* In Maryland, a majority of forest stands are fewer than 20 acres in size, and in Delaware, fewer than 50 acres.

* White oak is the most common mast-producing tree species, followed by chestnut oak and northern red oak in Maryland and blackgum and southern red oak in Delaware.

* Loblolly pine is the most common standing dead tree species.

* Red maple is the most common tree species, including both live and dead stems, with an observed cavity.

* Red maple is the most common understory woody-stemmed species, and blueberry is the most common shrub species. 


\section{Introduction}

Under the authority of the McSweeny-McNary Forest Research Act of 1928 and subsequent acts, including the Renewable Resources Planning Act of 1974 and the Renewable Resources Research Act of 1978, the USDA Forest Service conducts periodic forest inventories of all states to provide up-to-date information on the forest resource of the Nation. The initial inventory of Maryland's resources was conducted in 1950. The second was completed in 1964, and the third inventory, which began in 1975, was completed in 1976. This report presents forest wildlife-habitat data from the fourth Maryland inventory completed in 1986, the first inventory for which such data were collected. The initial inventory of Delaware's resources was conducted in 1956. The second inventory was completed in 1971. This report presents forest resource data from the third inventory completed in 1986. These inventories were a cooperative effort of the Maryland Forest, Parks and Wildlife Service, Delaware Department of Agriculture, Forestry Section, the USDA Soil Conservation Service, and the Northeastern Forest Experiment Station.

The Forest Inventory and Analysis Unit of the Northeastern Forest Experiment Station conducted the inventory on all forest land, developed the resource tables, and prepared this report.

The sampling procedure used during the current resurvey included the use of aerial photography, the remeasurement of a sample of the ground plots established in earlier inventories, and the establishment of new ground plots. The data collected were summarized using the FINSYS computer system developed at the Northeastern Forest Experiment Station.

In Maryland, this procedure required the classification of 18,355 new points and the reclassification of 550 points on aerial photographs from the third inventory into land-use and cubic-foot volume classes: the establishment of 602 (342 forest and 260 nonforest) new ground plot locations as a subsample of the new photo points; and the remeasurement of 550 (296 forest and 254 nonforest) plots from earlier inventories. In all, 1,152 plots were measured for an average of 1 plot for every 5,465 acres.
In Delaware, 122 (80 forest and 42 nonforest) plots from the earlier inventories were remeasured, 3,670 new photo points were classified and 122 photo points from the second inventory were reclassified into land-use and cubic-foot volume classes, and 118 (66 forest and 52 nonforest) new ground plot locations were established as a subsample of the new photo points.

The resurvey of Maryland and Delaware's forest resources involved several associated studies and considerable analysis. Forest area, numbers of trees, timber volume, biomass, and growth statistics have been published (Frieswyk and DiGiovanni 1989a,b). Reports discussing the states' private forest-land owners and its primary forest products industry are being prepared. Additional reports will contain detailed 1986 biomass statistics. Other information or additional summaries may be developed. For information about these, contact the Forest Inventory and Analysis Unit, USDA Forest Service, 370 Reed Road, Broomall, PA 19008 (phone 215-690-3029).

\section{Reliability of the Estimates}

The data in this report were based on a carefully designed sample of forest conditions throughout Maryland and Delaware. However, because the field crews did not measure every tree or every acre in these states, the data are estimates. The reliability of the estimating procedure can be judged by two important statistical measures: accuracy and precision. Among statisticians, accuracy refers to the success of estimating the true value; precision refers to the clustering of sample values about their own averages or to the variation among repeated samples. We are mainly interested in the accuracy of the inventory, but in most cases we can only measure its precision.

Although accuracy cannot be measured exactly, it can be checked. Preliminary tables are sent to other agencies and to outside experts familiar with the forest resources of Maryland and Delaware. If questions arise, the data are reviewed and reanalyzed to resolve the differences. Also, great care is taken to keep all sources of procedural error to a minimum by careful training of both field and office personnel, frequent in- 
spection of field and office work, and application of the most reliable inventory methods.

Because of the care exercised in the inventory process estimates of precision afford a reasonable measure of the inventory's adequacy. The precision of each estimate is described by its sampling error. Sampling errors are given with several tables in this report. The others are available upon request.

Briefly, here is an example of how the sampling error is used to indicate reliability: The estimate of timberland for Maryland is 2,424,000 acres. Its sampling error is 1.1 percent, or 26,664 acres. This means that if there are no errors in the procedure and we repeated the inventory in the same way, the odds are 2 to 1 (66 percent probability) that the estimate would be between $2,397,336$ and $2,450,664$ acres $(2,424,000+$ 26,664). Similarly, the odds are 19 to 1 (95 percent probability) that the estimate would be within 53,328 acres. It is worth noting that the state estimates have the smallest sampling errors and therefore are the most precise or reliable. County estimates are less reliable. In Maryland for example, the sampling error for timberland area at the state level is 1.1 percent; while the sampling error for Frederick County is 5.2 percent. In general, as the size of the estimate decreases in relation to the total, the sampling error, expressed as a percentage of the estimate, increases.

\section{Definition of Terms}

Agriculture/herbaceous land. Land with herbaceous plant cover, both grasses and/or forbs, including cropland, pasture land, and natural grass lands.

Aquatic Edge. An edge condition created when a terrestrial land use abuts a lake, pond, river, stream, or major wetland.

Bog/Marsh/Swamp. Land that has less than 10.0 percent stocking with live trees, and which characteristically supports low, generally herbaceous or shrubby vegetation, and which is intermittently covered with water during all seasons; includes tidal areas that are covered with salty or brackish water during high tides.
Browse. Forage resource; defined here as current twig growth of woody-stemmed plants occurring between 1 and 8 feet in height.

Browse-utilization class. Four levels of browse use; none, light (1-10 percent available), moderate (11-40 percent), and heavy (greater than 40 percent).

Cavity. A hollowed out space in a tree, either natural or faunal caused; frequently used as a nesting site or temporary refuge by many species of wildlife.

Commercial species. Tree species presently or prospectively suitable for industrial wood products. Excludes species of typically small size, poor form, or inferior quality, such as hawthorn and sumac.

Condition class. Classification of trees based on live or dead and condition of top of the tree (i.e., intact, broken, dead).

County and municipal lands. Lands owned by counties and local public agencies or municipalities or leased to them for 50 years or more.

Cropland. Land that currently supports agricultural crops including silage and feed grains, bare farm fields resulting from cultivation of harvest, and maintained orchards.

Cull tree. A live tree predominantly rotten or of rough form (see growing-stock trees).

Cultural land. Land with human development as the major land cover; includes industrial, commercial, and residential land uses.

Diameter at breast height (d.b.h.). The diameter outside bark of a standing tree measured at 4-1/2 feet above the ground.

Distribution. Percentage of plots where a species occurs.

Farmer-owned lands. Lands owned by farm operators, whether part of the farmstead or not. Excludes land leased by farm operators from nonfarm owners. 
Federal lands. Lands (other than National Forests) administered by Federal agencies.

Forest industry lands. Lands owned by companies or individuals operating primary wood-using plants.

Forest land. Land at least 10 percent stocked with trees of any size or that formerly had such tree cover and is not currently developed for nonforest use. The minimum area for classification of forest land is 1 acre.

Forest type. A classification of forest land by species that form a plurality of live tree basal-area stocking.

Forest-type group. A combination of forest types that share closely associated species or site requirements. The many forest types in Maryland and Delaware were combined into the following major forest-type groups (the descriptions apply to forests in Maryland and Delaware).

a. White/red pine--forests in which white pine, hemlock, or red pine make up the plurality of the stocking, singly or in combination; common associates include maple, oak, and yellow-poplar.

b. Spruce/fir--forests in which red spruce, northern white-cedar, balsam fir, white spruce, black spruce, or tamarack, singly or in combination, make up a plurality of the stocking; common associates include paper birch, red maple, aspen, white pine, hemlock, and sugar maple.

c. Loblolly!shortleaf pine group--forests in which loblolly, shortleaf, or other southern yellow pines (except longleaf or slash pine), singly or in combination, make up a plurality of the stocking; common associates include oaks, red maple, and blackgum.

d. Oak/pine--forests in which northern red oak or white ash, singly or in combination, make up a plurality of the stocking but where pines or eastern redcedar contribute 25 to 50 percent of the stocking; Virginia and loblolly pine, southern red oak, hickory, and blackgum are associates.

e. Oak/hickory--forests in which upland oaks, red maple (when associated with central hardwoods), or hawthorn, singly or in combination, make up a plurality of the stocking and in which white pine makes up less than 25 percent of the stocking; common associates include hard pine, ash, yellow-poplar, beech, blackgum, sugar maple, and red maple.

f. Oak/gum/cypress--bottomland forests in which wet-site oaks, sweetgum, or baldcypress, singly or in combination, make up a plurality of the stocking and in which pines make up less than 25 percent of the stocking; common associates include American elm, red maple, blackgum, and green ash.

g. Elm/ash/red maple--forests in which black ash, elm, red maple (when growing on wet sites), willow, or green ash, singly or in combination, make up a plurality of the stocking; common associates include bottomland oaks, blackgum, river birch, and silver maple.

h. Northern hardwoods--forests in which sugar maple, beech, yellow birch, red maple (when associated with northern hardwoods), pin cherry, or black cherry, singly or in combination, make up a plurality of the stocking; common associates include red maple, northern red oak, hemlock, white ash, and basswood.

Growing-stock trees. Live trees of commercial species classified as sawtimber, poletimber, saplings, or seedlings; that is, all live trees of commercial species except rough and rotten trees.

Hardwoods. Dicoîledonous trees, usually broad-leaved and deciduous.

Harvested cropland. All land from which crops were harvested or hay was cut, and all land in orchards, citrus groves, vineyards, and nursery and greenhouse products. 
Idle farmland. Former cropland or pasture that has not been tended within the last 2 years and that has less than 10.0 percent stocking with live trees (established seedlings or large trees), regardless of species.

Improved/maintained pasture. Land that is currently used and maintained for grazing (not including grazed cropland).

Importance value. Average of relative density and relative frequency of a species

Industrial and commercial land. Supply yards, parking lots, factories, etc.

Land area. (a) Bureau of Census: The area of dry land and land temporarily or partly covered by water, such as marshes, swamps, and river flood plains; streams, sloughs, estuaries, and canals less than $1 / 8$ statute mile wide; and lakes, reservoirs, and ponds fewer than 40 acres in area. (b) Forest Inventory and Analysis: same as (a) except that the minimum width of streams, etc. is 120 feet, and the minimum size of lakes, etc. is 1 acre.

Land use edge. A condition created by the juxtaposition of two differing land uses.

Mast. Seed produced by woody-stemmed, perennial plants, generally refers to soft (fruit) and hard (nuts) mast.

Mining and waste land. Surface mining, gravel pits, dumps.

Miscellaneous private lands. Privately owned lands other than forest-industry and farmerowned lands.

National Forest lands. Federal lands legally designated as National Forests or purchase units and other lands administered as part of the $\mathrm{Na}$ tional Forest System by the USDA Forest Service.

Noncommercial forest land. Productive-reserved, urban, and unproductive forest land.

Noncensus water. Streams/rivers between 120 feet and $1 / 8$ mile in width, and bodies of water between 1 and 40 acres in size. The Bureau of the Census classifies such water as land.
Noncommercial species. Tree species of typically small size, poor form, or inferior quality that normally do not develop into trees suitable for industrial wood products.

Nonforest land. Land that has never supported forests, or land formerly forested but not in nonforest use such as cropland, pasture, residential areas, and highways.

Nonstocked area. A stand-size class of forest land that is stocked with less than 10 percent of minimum full stocking with all live trees.

Other cropland. Includes cropland used for cover crops; legumes, soil-improvement grasses, but not harvested and not pastured; cropland on which all crops failed; cropland in summer fallow and idle cropland.

Other farmland. All nonforest land on a farm excluding cropland, pasture, and idle farmland; includes farm lanes, stock pens, and farmsteads.

Pasture land. Includes any pasture land other than cropland and woodland pasture. Can include lands which had applied lime fertilizer, seed, improved by irrigation, drainage, or control of weeds and brush.

Pastured cropland. Includes rotation pasture and grazing land that would have been used for crops without additional improvement.

Poletimber stands. A stand-size class of forest land that is stocked with at least 10 percent of minimum full stocking with all live trees with half or more of such stocking in poletimber or sawtimber trees or both, and in which the stocking of poletimber exceeds that of sawtimber.

Poletimber trees. Live trees of commercial species meeting regional specifications of soundness and form and at least 5.0 inches in d.b.h., but smaller than sawtimber trees.

Productive-reserved forest land. Forest land sufficiently productive to qualify as timberland, but withdrawn from timber utilization through statute, administrative designation, or exclusive use for Christmas tree production. 
Recreation site. Parks, campgrounds, playing fields, tracks, etc.

Relative density. Percentage of stems for a species.

Relative frequency. Percentage of plots where a species occurs. (Frequency of a species/total of frequencies of all species $\times 100$ )

Rights-of-way. Highways, pipelines, powerlines, canals.

Rotten trees. Live trees of commercial species that do not contain at least one 12-foot sawlog or two noncontiguous sawlogs, each 8 feet or longer, now or prospectively, and do not meet regional specifications for freedom from defect primarily because of rot; that is, when more than 50 percent of the cull volume in a tree is rotten.

Rough trees. (a) The same as rotten trees except that rough trees do not meet regional specifications for freedom from defect primarily because of roughness or poor form, and (b) all live trees of noncommercial species.

Saplings. Live trees 1.0 through 4.9 inches d.b.h.

Sapling-seedling stand's. A stand-size class of forest land that is stocked with at least 10 percent of minimum full stocking with all live trees with half or more of such stocking in saplings or seedlings or both.

Sawtimber stands. A stand-size class of forest land that is stocked with at least 10 percent of minimum full stocking with all live trees with half or more of such stocking in poletimber or sawtimber trees or both, and in which the stocking of sawtimber is at least equal to that of poletimber.

Sawtimber trees. Live trees of commercial species at least 9.0 inches d.b.h. for softwoods or 11.0 inches for hardwoods containing at least one 12-foot sawlog or two noncontiguous 8-foot sawlogs, and meeting regional specifications for freedom from defect.

Seedlings. Live trees less than 1.0 inch d.b.h. that are expected to survive.
Shrub. Woody-stemmed perennial plant, generally with no well-defined main stem and less than 12 feet in height at maturity; defined by species.

Shrub land. Land with shrub and/or tree cover and an obvious herbaceous understory; average canopy height of less than 25 feet and crown closure of less than 70 percent.

Single-family/custom house. House sheltering one family and immediately adjacent managed land.

Softwoods. Coniferous trees, usually evergreen and having needles or scalelike leaves.

Stand. A group of forest trees growing on forest land.

Stand area class. The area, contiguous to the plot, that is of the same overall stand size and major type group (hardwood, softwood, or uniform mixture of both).

Standing dead tree (snag). Woody stem more than 5.0 inches in diameter and 10 feet in height.

Stand-size class. A classification of forest land based on the size class (that is, seedlings, saplings, poletimber, or sawtimber) of all live trees in the area.

State land's. Lands owned by the State or leased to the State for 50 years or more.

Stocking. The degree of occupancy of land by trees, measured by basal area and/or number of trees in a stand compared to the basal area and/or number of trees required to fully use the growth potential of the land (or the stocking standard). In the Eastern United States this standard is 75 square feet of basal area per acre for trees 5.0 inches d.b.h. and larger, or its equivalent in numbers of trees per acre for seedlings and saplings.

Two categories of stocking are used:

All live trees--these are used to classify forest land, forest types, and stand size classes. 
Growing-stock trees--these are used to classify stand-size classes.

Stripmine. Area devoid of vegetation due to current or recent general excavation.

Timberland. Forest land producing or capable of producing crops of industrial wood (more than 20 cubic feet per acre per year) and not withdrawn from timber utilization (previously termed commercial forest land).

Tract/multiple family. Multiple individual residential units or attached units (e.g., apartment buildings, condominiums) and immediately adjacent managed land.

Transportation right-of-way. Land associated with highways and railroads.
Trees. Woody plants that have well-developed stems and are usually more than 12 feet in height at maturity; defined by species.

Unproductive forest land. Forest land that is incapable of producing 20 cubic feet per acre per year of industrial wood under natural conditions, because of adverse site conditions.

Urban forest land. Noncommercial forest land within urban areas that is completely surrounded by urban development (not parks), whether commercial, industrial, or residential.

Utility right-of-way. Land associated with pipeline and electric transmission lines, identified only if vegetative cover differs from adjacent land use.

Windbreak/hedgerow. Linear areas, fewer than 120 feet in width, with predominantly tree and/or shrub vegetation. 


\section{References}

Brooks, Robert T.; Sykes, Karen J. 1984. Sampling land use edge from aerial photographs-line transects vs. circular pattern. Res. Note NE-321. Broomall, PA: U.S. Department of Agriculture, Forest Service, Northeastern Forest Experiment Station. 6 p.

Ferguson, Roland H. 1959. The timber resources of Delaware. Upper Darby, PA: U.S. Department of Agriculture, Forest Service, Northeastern Forest Experiment Station; 1959. 30 p.

Ferguson, Roland H. 1967. The timber resources of Maryland. Res. Bull. NE-7. Upper Darby, PA: U.S. Department of Agriculture, Forest Service, Northeastern Forest Experiment Station. $93 \mathrm{p}$.

Ferguson, Roland H.; Mayer, Carl E. 1974. The timber resources of Delaware. Res. Bull. NE-32. Upper Darby, PA: U.S. Department of Agriculture, Forest Service, Northeastern Forest Experiment Station. 42 p.

Frieswyk, Thomas W.; DiGiovanni, Dawn M. 1989a. Forest statistics for Maryland--1976 and 1986. Res. Bull. NE-107. Broomall, PA: U.S. Department of Agriculture, Forest Service, Northeastern Forest Experiment Station. 157 p.

Frieswyk, Thomas W.; DiGiovanni, Dawn M. 1989b. Forest statistics for Delaware--1972 and 1986. Res. Bull. NE-109. Broomall, PA: U.S. Department of Agriculture, Forest Service, Northeastern Forest Experiment Station. 86 p.

Northeastern Forest Experiment Station. 1955. The timber resource in Maryland. Upper Darby, PA: U. S. Department of Agriculture, Forest Service, Northeastern Forest Experiment Station. $41 \mathrm{p}$.

Powell, Douglas S., Kingsley, Neal P. 1980. The Forest resources of Maryland. Res. Bull. NE-61. Upper Darby, PA: U.S. Department of Agriculture, Forest Service, Northeastern Forest Experiment Station. 103 p. 
Trees, shrubs, and vines of the Northeast

TREES

\begin{tabular}{|c|c|c|}
\hline Common Name & Genus & Species \\
\hline fir $s p$ & Abies & sp. \\
\hline balsam fir & Abies & balsamea \\
\hline Atlantic white-cedar & Chamaecyparis & thyoides \\
\hline eastein redcedar & Juniperus & virginiana \\
\hline larch (introduced) & Larix & sp. \\
\hline tamarack (native) & L. & laricina \\
\hline Norway spruce & Picea & abie \\
\hline white spruce & P. & glauca \\
\hline black spruce & P. & mariana \\
\hline blue spruce & P. & pungen \\
\hline red spruce & P. & rubens \\
\hline jack pine & Pinus & banksiana \\
\hline shortleaf pine & P. & echinata \\
\hline Table Mountain pine & P. & pungens \\
\hline red pine & P. & resinosa \\
\hline pitch pine & $P$. & rigida \\
\hline pond pine & P. & serotina \\
\hline eastern white pine & $P$. & strobus \\
\hline Scotch pine & P. & sylvestris \\
\hline loblolly pine & P. & taeda \\
\hline Virginia pine & P. & virginiana \\
\hline Austrian pine & P. & nigra \\
\hline Douglas fir & Pseudotsuga & menziesii \\
\hline baldcypress & Taxodium & distichum \\
\hline northern white-cedar & Thuja & occidentalis \\
\hline eastern hemlock & Tsuga & canadensis \\
\hline maple species & Acer & sp. \\
\hline boxelder & A. & negundo \\
\hline black maple & A. & nigrum \\
\hline striped maple & A. & pensylvanicum \\
\hline red maple & A. & rubrum \\
\hline silver maple & A. & saccharinum \\
\hline sugar maple & A. & saccharum \\
\hline mountain maple & A. & spicatum \\
\hline buckeye, horsechestnut & Aesculus & $\mathrm{sp}$. \\
\hline Ohio buckeye & A. & glabra \\
\hline yellow buckeye & A. & octandra \\
\hline ailanthus & Ailanthus & altissima \\
\hline serviceberry & Amelanchier & sp. \\
\hline pawpaw & Asimina & triloba \\
\hline yellow birch & Betula & alleghaniensis \\
\hline sweet birch & B. & lenta \\
\hline
\end{tabular}


Trees, shrubs, and vines of the Northeast (continued)

TREES (continued)

Common Name

Genus

Species

river birch

paper birch

B.

B.

gray birch

B.

American hornbeam, musclewood

Carpinus

hickory

water hickory

Carya

C.

bitternut hickory

C.

pignut hickory

C.

pecan

C.

shellbark hickory

C.

shagbark hickory

mockernut hickory

American chestnut

catalpa

sugarberry

hackberry

eastern redbud

yellowwood

flowering dogwood

hawthorn

common persimmon

American beech

white ash

black ash

green ash

pumpkin ash

blue ash

honeylocust

Kentucky coffeetree

American holly

butternut

black walnut

sweetgum

yellow-poplar

Osage-orange

magnolia

cucumbertree

sweetbay

apple sp.

mulberry

white mulberry

red mulberry

C.

C.

Castanea

Catalpa

Celtis

C.

Cercis

Cladrastis

Cornus

Crataegus

Diospyros

Fagus

Fraxinus

F.

F.

F.

F.

Gleditsia

Gymnocladus

Ilex

Juglans

J.

Liquidambar

Liriodendron

Maclura

Magnolia

$M$.

M.

Malus

Morus

$M$.

M. nigra

papyrifera

populifolia

caroliniana

sp.

aquatica

cordiformis

glabra

illinoensis

laciniosa

ovata

tomentosa

dentata

$\mathrm{sp}$.

laevigata

occidentalis

canadensis

lutea

florida

sp.

virginiana

grandifolia

americana

nigra

pennsylvanica

profunda

quadrangulata

triacanthos

dioicus

opaca

cinerea

nigra

styraciflua

tulipifera

pomifera

sp.

acuminata

virginiana

$\mathrm{sp}$.

$\mathrm{sp}$.

alba

rubra 
Trees, shrubs, and vines of the Northeast (continued)

TREES (continued)

Common Name

water tupelo

blackgum

swamp tupelo

eastern hophornbeam, ironwood

sourwood

Paulownia, Empress tree

sycamore

balsam poplar

eastern cottonwood

bigtooth aspen

swamp cottonwood

quaking aspen

cherry, plum

pin cherry

black cherry

chokecherry

oak

white oak

swamp white oak

scarlet oak

northern pin oak

southern red oak

cherrybark oak, swamp red oak

bear oak, scrub oak

shingle oak

laurel oak

overcup oak

bur oak

blackjack oak

swamp chestnut oak

chinkapin oak

water oak

pin oak

willow oak

chestnut oak

northern red oak

Shumard oak

post oak

black oak

black locust

willow
Genus

Species

Nyssa

N.

N.

Ostrya

Oxydendrum

Paulownia

Platanus

Populus

P.

$P$.

P.

P.

Prunus

P.

P.

$P$.

Q.

Quercus

Q.

Q.

Q.

Q.

Q.

Q.

Q.

Q.

Q.

Q.

Q.

Q.

Q.

Q.

$Q$.

Q.

Q.

Q.

Q.

Q.

Q.

Robinia

Salix aquatica

sylvatica

biflora

virginiana

arboreum

tomentosa

occidentalis

balsamifera

deltoides

grandidentata

heterophylla

tremuloides

sp.

pensylvanica

serotina

virginiana

sp.

alba

bicolor

coccinea

ellipsoidalis

falcata var.

falcata

falcata var.

pagodaefolia

ilicifolia

imbricaria

laurifolia

lyrata

macrocarpa

marilandica

michauxii

muehlenbergii

nigra

palustris

phellos

prinus

rubra

shumardii

stellata

velutina

psuedoacacia

sp. 
Trees, shrubs, and vines of the Northeast (continued)

TREES

Common ivame
black willow
sassafras
American mountain-ash
European mountain-ash
American basswood
white basswood
elm
winged elm
American elm
slippery elm
rock elm
unknown or not listed

Genus

Species

$S$.

Sassafras albidum

Sorbus americana

S.

Tilia

$T$.

Ulmus

$U$.

U.

U.

$U$.

aucuparia

americana

heterophylla

sp.

lata

americana

rubra

thomasii

\section{DECIDUOUS SHRUBS}

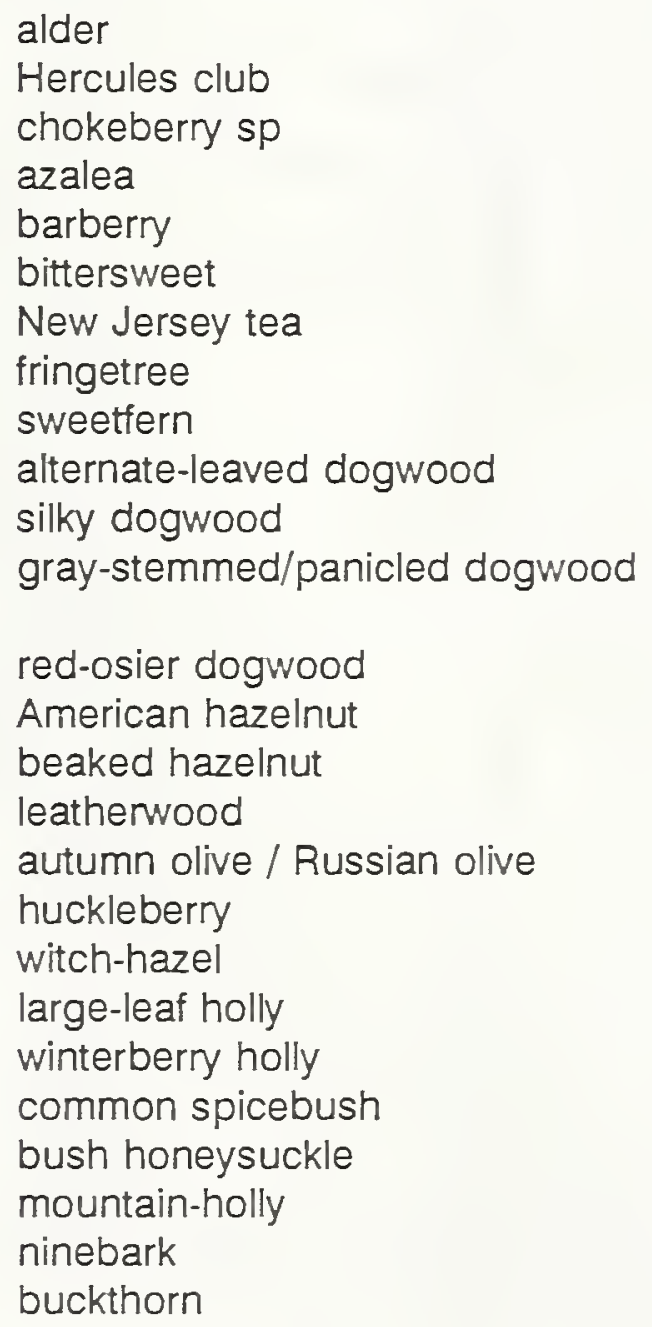

$\begin{array}{ll}\text { Alnus } & \text { sp. } \\ \text { Aralia } & \text { spinosa } \\ \text { Aronia } & \text { sp. } \\ \text { Azalea } & \text { sp. } \\ \text { Berberis } & \text { sp. } \\ \text { Celastrus } & \text { scandens } \\ \text { Cleanothus } & \text { americanus } \\ \text { Chionanthus } & \text { virginicus } \\ \text { Comptonia } & \text { peregrina } \\ \text { Cornus } & \text { alternifolia } \\ \text { C. } & \text { amomum (obliqua) } \\ \text { C. } & \text { racemosa } \\ & \text { (paniculata) } \\ \text { C. } & \text { stolonifera } \\ \text { Corylus } & \text { americana } \\ \text { C. } & \text { cornuta (rostrata) } \\ \text { Dirca } & \text { palustris } \\ \text { Elaeagnus } & \text { angustifolia } \\ \text { Gaylussacia } & \text { sp. } \\ \text { Hamamelis } & \text { virginiana } \\ \text { Ilex } & \text { montana (monticola) } \\ \text { I. } & \text { verticillata } \\ \text { Lindera } & \text { benzoin } \\ \text { Lonicera } & \text { sp. } \\ \text { Nemopanthus } & \text { mucronatus } \\ \text { Physocarpus } & \text { opulifolius } \\ \text { Rhamnus } & \text { sp. } \\ \end{array}$


Trees, shrubs, and vines of the Northeast (continued)

DECIDUOUS SHRUBS (continued)

Common Name

winged sumac

smooth sumac

staghorn sumac

poison sumac

currant, gooseberry

rose

brier, bramble, dewberry

American elderberry

red-berried elderberry

spirea

American bladdernut

blueberry

viburnum

maple-leaved viburnum

hobblebush viburnum

wild raisin, withe-rod

arrowwood

nannyberry

blackhaw

highbush cranberry

common prickly-ash

unknown or not listed
Genus

Rhus

R.

R.

R.

Ribes

Rosa

Rubus

Sambucus

S.

Spirea

Staphylea

Vaccinium

Viburnum

v.

V.

V.

V.

V.

V.

V.

Zanthoxylum
Species

copallina

glabra

typhina

vernix

sp.

sp.

sp.

canadensis

racemosa

sp.

trifolia

sp.

sp.

acerifolium

alnifolium

cassinoides

dentatum

lentago

prunifolium

trilobum

americanum

\section{EVERGREEN SHRUBS}

common juniper

Canada yew

bog rosemary

sheep laurel

mountain laurel

Labrador tea

rhododendron

sweetleaf

unknown or not listed
Juniperus

Taxus

Andromeda

Kalmia

$\mathrm{K}$.

Ledum

Rhododendron

Symplocos communis canadensis glaucophylla angustifolia latifolia groenlandicum sp. tinctoria

\section{DWARF SHRUBS}

evergreen bearberry striped pipsissewa

pipsissewa

bunchberry

creeping snowberry

$\begin{array}{ll}\text { Arctostaphylos } & \text { uva-ursi } \\ \text { Chimaphila } & \text { maculata } \\ \text { C. } & \text { cisatlantica } \\ \text { Cornus } & \text { canadensis } \\ \text { Gaultheria } & \text { hispidula }\end{array}$


Trees, shrubs, and vines of the Northeast (continued)

DWARF SHRUBS (continued)

Common Name

Genus

Species

teaberry

partridgeberry

American cranberry

unknown or not listed

G.

Mitchella

Vaccinium

procumbens

repens

sp.

VINES

hog peanut

American bittersweet

clematis $\mathrm{sp}$.

vine honeysuckle

Virginia creeper

poison ivy

greenbrier

grape

unknown or not listed
Amphicarpa

Celastrus

Clematis

Lonicera

Parthenocissus

Rhus

Smilax

Vitis bracteata

scandens

sp.

sp.

quinquefolia radicans

$\mathrm{sp}$.

sp. 
Ecological Importance and Relative Distribution of Lesser Woody-Stemmed Species ${ }^{1}$, Maryland

\begin{tabular}{|c|c|c|c|c|}
\hline Species & $\begin{array}{l}\text { Relative } \\
\text { Density }\end{array}$ & $\begin{array}{r}\text { Relative } \\
\text { Frequency }\end{array}$ & $\begin{array}{r}\text { Importance } \\
\text { Value }\end{array}$ & Distribution \\
\hline Atlantic white-cedar & .02 & .06 & .04 & .62 \\
\hline Common juniper & .04 & .13 & .08 & 1.38 \\
\hline Eastern redcedar & .04 & .29 & .16 & 3.06 \\
\hline Tamarack & .01 & .02 & .01 & .16 \\
\hline Norway spruce & .03 & .05 & .04 & .46 \\
\hline White spruce & .01 & .02 & .01 & .16 \\
\hline Red spruce & .01 & .08 & .04 & .77 \\
\hline Shortleaf pine & .01 & .03 & .02 & .31 \\
\hline Table mountain pine & .01 & .03 & .02 & .31 \\
\hline Red pine & .05 & .10 & .08 & 1.08 \\
\hline Pitch pine & .05 & .25 & .15 & 2.60 \\
\hline Pond pine & .07 & .12 & .09 & 1.23 \\
\hline Eastern white pine & .06 & .32 & .19 & 3.37 \\
\hline Scotch pine & .07 & .17 & .12 & 1.84 \\
\hline Loblolly pine & 1.39 & 2.74 & 2.06 & 29.52 \\
\hline Virginia pine & .83 & 2.17 & 1.50 & 23.40 \\
\hline Austrian pine & .01 & .02 & .01 & .16 \\
\hline Baldcypress & .01 & .03 & .02 & .31 \\
\hline Eastern hemlock & .08 & .29 & .18 & 3.06 \\
\hline Boxelder & .11 & .13 & .12 & 1.38 \\
\hline Striped maple & .34 & .30 & .32 & 3.22 \\
\hline Red maple & 6.38 & 7.24 & 6.81 & 78.14 \\
\hline Silver maple & .05 & .09 & .07 & .92 \\
\hline Sugar maple & .67 & .85 & .76 & 9.18 \\
\hline Mountain maple & .08 & .06 & .07 & .62 \\
\hline Ailanthus & .02 & .09 & .06 & .92 \\
\hline Alder species & .82 & .19 & .50 & 1.99 \\
\hline Hercules club & .38 & .56 & .47 & 5.97 \\
\hline Serviceberry & .88 & .88 & .88 & 9.49 \\
\hline Evergreen bearberry² & -- & -- & -. & .31 \\
\hline Chokeberry species & .01 & .02 & .01 & .16 \\
\hline Azalea species & 1.96 & .78 & 1.37 & 8.41 \\
\hline Common pawpaw & .98 & .50 & .74 & 5.36 \\
\hline Barberry & .02 & .02 & .02 & .16 \\
\hline Birch species & .07 & .02 & .01 & .16 \\
\hline Yellow birch & .07 & .17 & .12 & 1.84 \\
\hline Sweet birch & .48 & .80 & .64 & 8.57 \\
\hline River birch & .05 & .19 & .12 & 1.99 \\
\hline Gray birch & .01 & .02 & .01 & .16 \\
\hline American hornbeam & .24 & .70 & .47 & 7.50 \\
\hline Hickory species & .72 & 2.95 & 1.84 & 31.81 \\
\hline Bitternut hickory & .03 & .13 & .08 & 1.38 \\
\hline Pignut hickory & .04 & .22 & .13 & 2.30 \\
\hline
\end{tabular}


Ecological Importance and Relative Distribution of Lesser Woody-Stemmed Species ${ }^{1}$, Maryland (continued)

\begin{tabular}{|c|c|c|c|c|}
\hline Species & $\begin{array}{l}\text { Relative } \\
\text { Density }\end{array}$ & $\begin{array}{r}\text { Relative } \\
\text { Frequency }\end{array}$ & $\begin{array}{r}\text { Importance } \\
\text { Value }\end{array}$ & Distribution \\
\hline Shagbark hickory & .02 & .39 & .20 & 4.13 \\
\hline Mockernut hickory & .03 & .26 & .15 & 2.76 \\
\hline American chestnut & .15 & .27 & .21 & 2.91 \\
\hline Catalpa & .01 & .03 & .02 & .31 \\
\hline American bittersweet ${ }^{2}$ & -- & -- & -- & .16 \\
\hline Hackberry & .10 & .19 & .15 & 1.99 \\
\hline Eastern redbud & .23 & .22 & .22 & 2.30 \\
\hline Fringetree & .02 & .06 & .04 & .62 \\
\hline Clematis species ${ }^{2}$ & -- & -- & -- & .92 \\
\hline Yellowwood & .01 & .02 & .01 & .16 \\
\hline Flowering dogwood & 1.67 & 2.21 & 1.94 & 23.86 \\
\hline Alternate-leaved dogwood & .02 & .06 & .04 & .62 \\
\hline Silky dogwood & .05 & .06 & .05 & .62 \\
\hline Panicled dogwood & .02 & .02 & .02 & .16 \\
\hline Canadian bunchberry ${ }^{2}$ & -- & -- & -- & .46 \\
\hline Hawthorn species & .34 & .57 & .45 & 6.12 \\
\hline American hazelnut & .04 & .02 & .03 & .16 \\
\hline Common persimmon & .06 & .43 & .25 & 4.59 \\
\hline American beech & .76 & 2.24 & 1.50 & 24.16 \\
\hline White ash & .94 & 1.60 & 1.27 & 17.28 \\
\hline Black ash & .01 & .02 & .01 & .16 \\
\hline Green ash & .04 & .25 & .14 & 2.60 \\
\hline Teaberry² $^{2}$ & -- & -- & -- & 6.43 \\
\hline Huckleberry & 2.29 & .53 & 1.41 & 5.66 \\
\hline Honeylocust & .01 & .02 & .01 & .16 \\
\hline Witch-hazel & .72 & .02 & .69 & 7.04 \\
\hline American holly & 1.39 & 2.54 & 1.96 & 27.38 \\
\hline Winterberry holly & .03 & .03 & .03 & .31 \\
\hline Butternut & .02 & .16 & .09 & 1.69 \\
\hline Black walnut & .04 & .47 & .25 & 5.05 \\
\hline Sheep laurel & .01 & .03 & .02 & .31 \\
\hline Mountain laurel & 1.18 & .78 & .98 & 8.41 \\
\hline Common spicebush & 1.83 & 1.12 & 1.48 & 12.08 \\
\hline Sweetgum & 2.60 & 4.42 & 3.51 & 47.71 \\
\hline Yellow-poplar & .95 & 3.08 & 2.02 & 33.19 \\
\hline Bush honeysuckle & .10 & .12 & .11 & 1.23 \\
\hline Vine honeysuckle 2 & - & - & - & 37.16 \\
\hline Magnolia & .01 & .03 & .02 & .31 \\
\hline Cumcumbertree & .12 & .22 & .17 & 2.30 \\
\hline Sweetbay & .32 & .56 & .44 & 5.97 \\
\hline Apple species & .02 & .08 & .05 & .77 \\
\hline Partridgeberry ${ }^{2}$ & - & -- & .00 & 14.38 \\
\hline Water tupelo & .14 & .30 & .22 & 3.22 \\
\hline
\end{tabular}


Ecological Importance and Relative Distribution of Lesser Woody-Stemmed Species ${ }^{1}$,Maryland (continued)

\begin{tabular}{|c|c|c|c|c|}
\hline Species & $\begin{array}{l}\text { Relative } \\
\text { Density }\end{array}$ & $\begin{array}{r}\text { Relative } \\
\text { Frequency }\end{array}$ & $\begin{array}{r}\text { Importance } \\
\text { Value }\end{array}$ & Distribution \\
\hline Black tupelo & 1.93 & 4.39 & 3.16 & 47.41 \\
\hline Eastern hophornbeam & .32 & .63 & .47 & 6.73 \\
\hline Paulownia & .01 & .10 & .06 & 1.08 \\
\hline Virginia creeper² & -- & -- & -- & 30.89 \\
\hline Ninebark & .01 & .02 & .02 & .16 \\
\hline American sycamore & .03 & .47 & .25 & 5.05 \\
\hline Eastern cottonwood & .01 & .02 & .01 & .16 \\
\hline Bigtooth aspen & .08 & .34 & .21 & 3.67 \\
\hline Quaking aspen & .01 & .02 & .02 & .16 \\
\hline Cherry species & .03 & .15 & .09 & 1.53 \\
\hline Pin cherry & .12 & .30 & .21 & 3.22 \\
\hline Black cherry & 2.72 & 3.37 & 3.05 & 36.40 \\
\hline Chokecherry & .06 & .16 & .11 & 1.69 \\
\hline White oak & 1.38 & 4.66 & 3.02 & 50.31 \\
\hline Swamp white oak & .06 & .43 & .25 & 4.59 \\
\hline Scalet oak & .15 & 1.53 & .84 & 16.52 \\
\hline Southern red oak & .62 & 2.84 & 1.73 & 30.59 \\
\hline Swamp red oak & .01 & .10 & .06 & 1.08 \\
\hline Scrub, bear oak & .15 & .06 & .10 & .62 \\
\hline Shingle oak & .01 & .02 & .01 & .16 \\
\hline Blackjack oak & .01 & .02 & .01 & .16 \\
\hline Swamp chestnut oak & .10 & .43 & .26 & 4.59 \\
\hline Chinkapin oak & .01 & .03 & .02 & .31 \\
\hline Water oak & .34 & .74 & .54 & 7.96 \\
\hline Pin oak & .05 & .46 & .25 & 4.90 \\
\hline Willow oak & .21 & 1.32 & .77 & 14.23 \\
\hline Chestnut oak & .96 & 1.99 & 1.47 & 21.41 \\
\hline Northern red oak & .92 & 3.52 & 2.22 & 37.93 \\
\hline Post oak & .02 & .16 & .09 & 1.69 \\
\hline Black oak & .46 & 2.53 & 1.49 & 27.22 \\
\hline Buckthorn species & .03 & .03 & .03 & .31 \\
\hline Rhododendron species & .38 & .12 & .25 & 1.23 \\
\hline Smooth sumac & .13 & .17 & .15 & 1.84 \\
\hline Staghorn sumac & .07 & .15 & .11 & 1.53 \\
\hline Poison Ivy² & -- & -- & -- & 31.81 \\
\hline Currant species & .11 & .12 & .12 & 1.23 \\
\hline Black locust & .66 & 1.69 & 1.17 & 18.20 \\
\hline Rose species & 3.77 & .77 & 2.27 & 8.26 \\
\hline Rubus species & 9.54 & 3.45 & 6.49 & 37.16 \\
\hline Willow species & .02 & .06 & .04 & .62 \\
\hline Black willow & .02 & .05 & .04 & .46 \\
\hline American elderberry & .12 & .16 & .14 & 1.69 \\
\hline
\end{tabular}




\section{Ecological Importance and Relative Distribution of Lesser Woody-Stemmed Species', Maryland (continued)}

\begin{tabular}{|c|c|c|c|c|}
\hline Species & $\begin{array}{l}\text { Relative } \\
\text { Density }\end{array}$ & $\begin{array}{r}\text { Relative } \\
\text { Frequency }\end{array}$ & $\begin{array}{r}\text { Importance } \\
\text { Value }\end{array}$ & Distribution \\
\hline Red-berried alder & .01 & .02 & .02 & .16 \\
\hline Sassafras & 1.82 & 2.47 & 2.15 & 26.61 \\
\hline Greenbrier2 & -- & -. & -- & 53.22 \\
\hline American mountain ash & .01 & .02 & .01 & .16 \\
\hline European mountain ash & .01 & .02 & .01 & .16 \\
\hline Spirea species & .06 & .03 & .05 & .31 \\
\hline American basswood & .03 & .27 & .15 & 2.91 \\
\hline Elm species & .18 & .47 & .33 & 5.05 \\
\hline American elm & .13 & .39 & .26 & 4.13 \\
\hline Slippery elm & .13 & .30 & .22 & 3.22 \\
\hline American bladdernut & .01 & .02 & .02 & .16 \\
\hline Blueberry & 16.14 & 4.11 & 10.12 & 44.35 \\
\hline Viburnum species & .55 & .37 & .46 & 3.98 \\
\hline Maple-leaved viburnum & 1.24 & .81 & 1.03 & 8.72 \\
\hline Hobblebush viburnun & .02 & .06 & .04 & .62 \\
\hline Wild raisin & .01 & .02 & .01 & .16 \\
\hline Arrowwood & 1.45 & 1.07 & 1.26 & 11.47 \\
\hline Nannyberry & .00 & .02 & .01 & .16 \\
\hline Blackhaw & .09 & .10 & .10 & 1.08 \\
\hline Highbush cranberry & .02 & .02 & .02 & .16 \\
\hline Grape² & -- & -- & -- & 14.99 \\
\hline Common prickly ash & .01 & .02 & .01 & .16 \\
\hline Unknown vine ${ }^{2}$ & -- & -- & -- & 9.03 \\
\hline Unknown dwarf shrub² & -- & -- & -- & 9.03 \\
\hline Unknown deciduous shrub & 19.23 & 3.22 & 11.23 & 34.71 \\
\hline Unknown evergreen shrub & .01 & .02 & .01 & .16 \\
\hline Unknown tree & .51 & 1.34 & .92 & 14.38 \\
\hline
\end{tabular}

${ }^{1}$ Includes shrub and vine species and tree stems less than 5.0 inches d.b.h. 2Not included in calculations of importance value. 


\section{Ecological Importance and Relative Distribution of Lesser Woody-Stemmed}

Species $^{1}$, Delaware

\begin{tabular}{|c|c|c|c|c|}
\hline Species & $\begin{array}{l}\text { Relative } \\
\text { Density }\end{array}$ & $\begin{array}{r}\text { Relative } \\
\text { Frequency }\end{array}$ & $\begin{array}{r}\text { Importance } \\
\text { Value }\end{array}$ & Distribution \\
\hline Atlantic white-cedar & .03 & .10 & .06 & 1.00 \\
\hline Eastern redcedar & .04 & .28 & .16 & 2.98 \\
\hline Pond pine & .02 & .47 & .25 & 4.96 \\
\hline Eastern white pine & .01 & .10 & .05 & 1.00 \\
\hline Loblolly pine & 2.82 & 4.73 & 3.77 & 50.50 \\
\hline Virginia pine & .92 & 2.69 & 1.80 & 28.72 \\
\hline Eastern hemlock & .02 & .10 & .06 & 1.00 \\
\hline Boxelder & .20 & .38 & .29 & 3.97 \\
\hline Red maple & 6.50 & 7.97 & 7.23 & 85.15 \\
\hline Silver maple & .04 & .10 & .07 & 1.00 \\
\hline Sugar maple & .02 & .10 & .06 & 1.00 \\
\hline Ailanthus & .01 & .10 & .05 & 1.00 \\
\hline Alder species & .05 & .10 & .07 & 1.00 \\
\hline Hercules club & .78 & 1.49 & 1.14 & 15.85 \\
\hline Serviceberry & .23 & .47 & .35 & 4.96 \\
\hline Chokeberry species & .04 & .10 & .07 & 1.00 \\
\hline Azalea species & 1.49 & 1.67 & 1.58 & 17.53 \\
\hline Common pawpaw & .52 & .10 & .31 & 1.00 \\
\hline Barberry & .05 & .10 & .08 & 1.00 \\
\hline Sweet birch & .03 & .10 & .06 & 1.00 \\
\hline River birch & .01 & .10 & .05 & 1.00 \\
\hline American hornbeam & .04 & .28 & .16 & 2.98 \\
\hline Hickory species & .19 & 1.67 & .93 & 17.83 \\
\hline Bitternut hickory & .01 & .19 & .10 & 1.99 \\
\hline Pignut hickory & .03 & .19 & .11 & 1.99 \\
\hline Clematis species ${ }^{2}$ & -- & -- & -- & 2.98 \\
\hline Sweetfern & .25 & .10 & .17 & 1.00 \\
\hline Flowering dogwood & .68 & 1.49 & 1.08 & 15.85 \\
\hline Silky dogwood & .37 & .10 & .24 & 1.00 \\
\hline Round-leaved dogwood & .12 & .10 & .11 & 1.00 \\
\hline Panicled dogwood & .07 & .10 & .08 & 1.00 \\
\hline American hazelnut & .02 & .10 & .06 & 1.00 \\
\hline Common persimmon & .19 & .56 & .38 & 5.95 \\
\hline American beech & .25 & 1.39 & .82 & 14.86 \\
\hline White ash & .05 & .38 & .21 & 3.97 \\
\hline Green ash & .01 & .28 & .15 & 2.98 \\
\hline Teaberry $^{2}$ & -. & -- & -- & 1.00 \\
\hline Witch-hazel & .05 & .19 & .12 & 1.99 \\
\hline American holly & 2.51 & 4.08 & 3.30 & 43.57 \\
\hline Black walnut & .02 & .38 & .20 & 3.97 \\
\hline Sheep laurel & .20 & .10 & .15 & 1.00 \\
\hline Mountain laurel & .02 & .10 & .06 & 1.00 \\
\hline Common spicebush & 1.06 & .65 & .86 & 6.94 \\
\hline
\end{tabular}


Ecological Importance and Relative Distribution of Lesser Woody-Stemmed Species ${ }^{1}$, Delaware (continued)

\begin{tabular}{|c|c|c|c|c|}
\hline Species & $\begin{array}{l}\text { Relative } \\
\text { Density }\end{array}$ & $\begin{array}{r}\text { Relative } \\
\text { Frequency }\end{array}$ & $\begin{array}{r}\text { Importance } \\
\text { Value }\end{array}$ & Distribution \\
\hline Sweetgum & 4.06 & 6.95 & 5.50 & 74.26 \\
\hline Yellow-poplar & .76 & 2.78 & 1.77 & 29.71 \\
\hline Vine honeysuckle 2 & -- & -- & -- & 33.67 \\
\hline Magnolia & .02 & .10 & .06 & 1.00 \\
\hline Sweetbay & .83 & .92 & .88 & 9.91 \\
\hline Partridgeberry² & - & - & -- & 23.77 \\
\hline Mulberry species & .01 & .19 & .10 & 1.99 \\
\hline Water tupelo & .17 & .28 & .23 & 2.98 \\
\hline Black tupelo & 1.84 & 5.56 & 3.70 & 59.41 \\
\hline Virginia creeper ${ }^{2}$ & -- & -- & -- & 39.61 \\
\hline American sycamore & .01 & .10 & .05 & 1.00 \\
\hline Swamp cottonwood & .07 & .10 & .09 & 1.00 \\
\hline Pin cherry & .02 & .10 & .06 & 1.00 \\
\hline Black cherry & 2.23 & 3.25 & 2.74 & 34.66 \\
\hline White oak & .82 & 5.38 & 3.10 & 57.43 \\
\hline Swamp white oak & .02 & .28 & .15 & 2.98 \\
\hline Scarlet oak & .22 & 1.58 & .90 & 16.84 \\
\hline Southern red oak & .62 & 3.15 & 1.89 & 33.67 \\
\hline Blackjack oak & .03 & .10 & .06 & 1.00 \\
\hline Swamp chestnut oak & .06 & .56 & .31 & 5.95 \\
\hline Water oak & .82 & 2.41 & 1.62 & 25.75 \\
\hline Pin oak & .10 & .75 & .43 & 7.93 \\
\hline Willow oak & .20 & 2.97 & 1.58 & 31.69 \\
\hline Chestnut oak & .07 & .47 & .27 & 4.96 \\
\hline Northern red oak & .34 & 1.86 & 1.10 & 19.81 \\
\hline Post oak & .02 & .28 & .15 & 2.98 \\
\hline Black oak & .20 & 1.30 & .75 & 13.87 \\
\hline Buckthorn species & .08 & .19 & .13 & 1.99 \\
\hline Smooth sumac & .38 & .28 & .33 & 2.98 \\
\hline Poison ivy? & -- & -- & -- & 40.60 \\
\hline Currant species & .29 & .19 & .24 & 1.99 \\
\hline Black locust & .01 & .19 & .10 & 1.99 \\
\hline Rose species & .45 & .38 & .41 & 3.97 \\
\hline Rubus species & 3.76 & 2.23 & 2.99 & 23.77 \\
\hline Willow species & .02 & .10 & .06 & 1.00 \\
\hline Black willow & .01 & .10 & .05 & 1.00 \\
\hline Sassafras & 1.80 & 3.89 & 2.85 & 41.59 \\
\hline Greenbrier & -- & -- & - & 82.18 \\
\hline American elm & .07 & .38 & .22 & 3.97 \\
\hline Slippery elm & .04 & .19 & .12 & 1.99 \\
\hline Blueberry & 17.92 & 4.63 & 11.28 & 49.51 \\
\hline Viburnum species & .26 & .38 & .32 & 3.97 \\
\hline Maple-leaved virburnum & .80 & .47 & .63 & 4.96 \\
\hline
\end{tabular}


Ecological Importance and Relative Distribution of Lesser Woody-Stemmed Species', Delaware (continued)

\begin{tabular}{lrrrr}
\hline \multicolumn{1}{c}{ Species } & $\begin{array}{c}\text { Relative } \\
\text { Density }\end{array}$ & $\begin{array}{r}\text { Relative } \\
\text { Frequency }\end{array}$ & $\begin{array}{r}\text { Importance } \\
\text { Value }\end{array}$ & Distribution \\
\hline Arrowwood & 2.36 & 2.60 & 2.48 & 27.73 \\
Blackhaw & .05 & .10 & .07 & 1.00 \\
Grape 2 & -- & -- & -- & 17.83 \\
Unknown vine 2 & -- & -- & -- & 9.91 \\
Unknown dwarf shrub ${ }^{2}$ & -- & 6.67 & 22.26 & 21.79 \\
Unknown deciduous shrub & 37.85 & .10 & .06 & 71.29 \\
Unknown evergreen shrub & .02 & 2.23 & 1.50 & 23.77 \\
Unknown tree & .77 & & & \\
\hline
\end{tabular}

IIncludes shrub and vine species and tree stems less than 5.0 inches d.b.h.

${ }^{2}$ Not included in calculations of importance value. 
Ecological Importance and Relative Distribution of Trees $5.0+$ d.b.h. by Species, Maryland

Species

Relative
Density

Relative

Relative

Importance

Dominance

Frequency

Value

Distribution

Atlantic white-cedar

Eastern redcedar

Tamarack

Norway spruce

Red spruce

Shortleaf pine

Table mountain pine

Red pine

Pitch pine

Pond pine

Eastern white pine

Scotch pine

Loblolly pine

Virginia pine

Austrian pine

Baldcypress

Eastern hemlock

Boxelder

Striped maple

Red maple

Silver maple

Sugar maple

Ailanthus

Serviceberry

Yellow birch

Sweet birch

River birch

Gray birch

American hornbeam

Hickory species

Bitternut hickory

Pignut hickory

Shagbark hickory

Mockernut hickory

American chestnut

Catalpa

Hackberry

Eastern redbud

Flowering dogwood

Hawthorn species

Common persimmon

American beech
.02

.18

.04

.11

.11

.01

.03

1.66

.27

.09

.60

.12

11.54

7.05

.01

.04

.49

.16

.03

13.18

.25

1.53

.04

.14

.14

.81

.14

.01

.12

2.46

.02

.22

.36

.14

.03

.00

.19

.07

.25

.04

.21

2.56
.03

.13

.05

.07

.07

.01

.02

.78

.21

.08

.50

.07

10.26

5.57

.01

.09

.62

.11

.02

11.51

.42

1.44

.03

.07

.12

.71

.18

.01

.53

2.28

.05

.23

.31

.14

.02

.00

.16

.04

.08

.02

.12

3.06
.08

.31

.03

.08

.08

.06

.06

.16

.41

.18

.43

.18

4.34

3.49

.03

.06

.46

.16

.06

9.87

.13

1.26

.11

.23

.21

1.06

.23

.03

.30

3.81

.16

.33

.66

.36

.03

.03

.26

.08

.53

.03

.53

3.18
.46

1.84

.16

.46

.46

.31

.31

.92

2.45

1.08

2.60

$+.08$

26.46

21.26

.16

.31

2.76

.92

.31

60.25

.77

7.65

.62

1.38

1.23

5.43

1.38

.16

3.22

23.25

.92

1.99

3.98

2.15

.16

.16

1.53

.45

3.22

.16

3.22

19.42 
Ecological Importance and Relative Distribution of Trees 5.0+ d.b.h. by Species, Maryland (continued)

\begin{tabular}{|c|c|c|c|c|c|}
\hline Species & $\begin{array}{l}\text { Relative } \\
\text { Density }\end{array}$ & $\begin{array}{r}\text { Relative } \\
\text { Dominance }\end{array}$ & $\begin{array}{r}\text { Relative } \\
\text { Frequency }\end{array}$ & $\begin{array}{r}\text { Importance } \\
\text { Value }\end{array}$ & Distribution \\
\hline White ash & 1.51 & 1.38 & 2.08 & 1.66 & 12.70 \\
\hline Black ash & .01 & .01 & .03 & .02 & .16 \\
\hline Green ash & .36 & .31 & .31 & .32 & 1.84 \\
\hline American holly & 1.49 & .73 & 1.81 & 1.34 & 11.01 \\
\hline Butternut & .07 & .07 & .23 & .12 & 1.38 \\
\hline Black walnut & .38 & .44 & .81 & .54 & 4.90 \\
\hline Sweetgum & 7.91 & 6.89 & 6.84 & 7.21 & 41.75 \\
\hline Yellow-poplar & 4.90 & 8.46 & 5.09 & 6.15 & 31.04 \\
\hline Magnolia & .04 & .02 & .03 & .03 & .16 \\
\hline Cumcumbertree & .05 & .05 & .21 & .10 & 1.23 \\
\hline Sweetbay & .21 & .11 & .31 & .21 & 1.84 \\
\hline Rpple species & .04 & .02 & .08 & .05 & .46 \\
\hline Water tupelo & .28 & .28 & .41 & .32 & 2.45 \\
\hline Black tupelo & 3.79 & 3.20 & 5.54 & 4.17 & 33.80 \\
\hline Eastern hophornbeam & .19 & .09 & .33 & .20 & 1.99 \\
\hline Paulownia & .11 & .08 & .18 & .12 & 1.08 \\
\hline American sycamore & .33 & .75 & .83 & .64 & 5.05 \\
\hline Eastern cottonwood & .02 & .02 & .03 & .02 & .16 \\
\hline Bigtooth aspen & .52 & .35 & .51 & .46 & 3.06 \\
\hline Quaking aspen & .02 & .02 & .03 & .02 & .16 \\
\hline Cherry species & .10 & .08 & .18 & .12 & 1.08 \\
\hline Pin cherry & .18 & .08 & .18 & .15 & 1.08 \\
\hline Black cherry & 2.46 & 2.00 & 3.03 & 2.50 & 18.51 \\
\hline Chokecherry & .03 & .02 & .08 & .04 & .46 \\
\hline White oak & 8.23 & 8.75 & 7.44 & 8.14 & 45.42 \\
\hline Swamp white oak & .28 & .35 & .66 & .43 & 3.98 \\
\hline Scarlet oak & 1.50 & 1.73 & 2.53 & 1.92 & 15.45 \\
\hline Southern red oak & 2.17 & 2.88 & 3.96 & 3.00 & 24.16 \\
\hline Swamp red oak & .13 & .10 & .16 & .13 & .92 \\
\hline Shingle oak & .02 & .01 & .03 & .02 & .16 \\
\hline Swamp chestnut oak & .25 & .43 & .61 & .43 & 3.67 \\
\hline Chinkapin oak & .03 & .05 & .06 & .05 & .31 \\
\hline Water oak & .74 & .51 & .93 & .73 & 5.66 \\
\hline Pin oak & .21 & .35 & .73 & .43 & 4.44 \\
\hline Willow oak & .72 & .89 & 1.68 & 1.10 & 10.25 \\
\hline Chestnut oak & 5.27 & 6.07 & 3.03 & 4.88 & 20.19 \\
\hline Northern red oak & 3.81 & 6.00 & 5.34 & 5.05 & 32.57 \\
\hline Post oak & .11 & .09 & .26 & .15 & 1.53 \\
\hline Black oak & 2.31 & 3.75 & 3.76 & 3.27 & 22.94 \\
\hline Black locust & 1.88 & 1.57 & 2.08 & 1.84 & 12.70 \\
\hline Willow species & .08 & .05 & .08 & .07 & .46 \\
\hline Black willow & .03 & .03 & .06 & .04 & .31 \\
\hline Sassafras & .73 & .41 & 1.21 & .78 & 7.34 \\
\hline
\end{tabular}


Ecological Importance and Relative Distribution of Trees $5.0+$ d.b.h. by Species, Maryland (continued)

\begin{tabular}{lrrrrr}
\hline \multicolumn{1}{c}{ Species } & $\begin{array}{c}\text { Relative } \\
\text { Density }\end{array}$ & $\begin{array}{c}\text { Relative } \\
\text { Dominance }\end{array}$ & $\begin{array}{r}\text { Relative } \\
\text { Frequency }\end{array}$ & $\begin{array}{r}\text { Importance } \\
\text { Value }\end{array}$ & Distribution \\
\hline American mtn. ash & .01 & .01 & .03 & .02 & .16 \\
American basswood & .32 & .35 & .46 & .38 & .76 \\
Elm species & .51 & .33 & .58 & .47 & 3.52 \\
American elm & .21 & .20 & .46 & .24 & 2.76 \\
Slippery elm & .17 & .19 & .36 & .19 & 2.15 \\
Unknown tree & .13 & .12 & .33 & & 1.99 \\
\hline
\end{tabular}


Ecological Importance and Relative Distribution of Trees 5.0+ d.b.h. by Species, Delaware

\begin{tabular}{|c|c|c|c|c|}
\hline Species & $\begin{array}{l}\text { Relative } \\
\text { Density }\end{array}$ & $\begin{array}{r}\text { Relative } \\
\text { Dominance }\end{array}$ & $\begin{array}{r}\text { Relative } \\
\text { Frequency }\end{array}$ & $\begin{array}{r}\text { Importance } \\
\text { Value }\end{array}$ \\
\hline
\end{tabular}

Pond pine

Eastern white pine

Loblolly pine

Virginia pine

Eastern hemlock

Boxelder

Red maple

Ailanthus

Sweet birch

River birch

American hornbeam

Hickory species

Bitternut hickory

Pignut hickory

Flowering dogwood

Common persimmon

American beech

White ash

Green ash

American holly

Black walnut

Sweetgum

Yellow-poplar

Mulberry species

Water tupelo

Black tupelo

American sycamore

Swamp cottonwood

Black cherry

White oak

Swamp white oak

Scarlet oak

Willow oak

Chestnut oak

Northern red oak

Post oak

Black oak

Black locust

Black willow

Sassafras

American elm

Slippery elm

Unknown tree

\section{.37}

.02

19.40

5.48

.03

.23

21.72

.17

.06

.03

.33

1.06

.26

.06

.65

.20

1.23

.53

.65

2.57

.15

12.50

1.35

.05

.19

5.89

.04

.56

2.94

8.24

.07

1.42

2.21

.23

.90

.21

.66

.17

.31

.71

.08

.03

.02
.38

.05

19.55

4.97

.03

.22

18.37

.15

.11

.03

.12

.77

.24

.06

.28

.13

1.43

.60

.65

1.19

.34

12.07

3.39

.08

.20

4.77

.15

.32

3.06

9.97

.22

1.45

3.40

.55

1.78

.30

.77

.17

.25

.48

.07

.01

.02
.72

.18

7.38

4.32

.18

.36

11.34

.18

.18

.18

.54

2.70

.36

.36

1.44

.54

1.80

.72

.54

3.96

.54

11.16

4.14

.36

.36

7.74

.18

.18

2.52

8.64

.36

1.74

4.86

.54

3.06

.54

1.98

.36

.18

1.80

.36

.18

.18
3.97

1.00

40.60

23.77

1.00

1.99

62.38

1.00

1.00

1.00

2.98

14.86

1.99

1.99

7.93

2.98

9.91

3.97

2.98

21.79

2.98

61.39

22.78

1.99

1.99

42.58

1.00

1.00

13.87

7.53

1.99

12.88

26.74

2.98

16.84

2.98

10.90

1.99

1.00

9.91

1.99

1.00

1.00 


\section{Metric Equivalents}

1 acre $=4,046.86$ meters $^{2}$ or 0.404686 hectares

1,000 acres $=404.686$ hectares

$1,000,000$ acres $=404,686$ hectares

1 inch $=2.54$ centimeters or 0.0254 meters

1 foot $=30.48$ centimeters or 0.3048 meters

Breast height $=1.4$ meters above ground level

1 mile $=1.609$ kilometers

1 square foot $=929.03$ centimeters $^{2}$ or 0.929

meters $^{2}$

1 square foot per acre basal area $=$

0.229568 meters $^{2} /$ hectares 
12. Number of seedlings, saplings, and shrubs on timberland by species and

Maryland tables are divided into six major sections: (1) State, (2) Central Unit, (3) Lower Eastern Shore Unit, (4) Southern Unit, (5) Western Unit, and (6) County. Delaware tables are divided into two major sections: (1) State and (2) County.

\section{MARYLAND}

\section{State Tables}

1. Land area by county and land class, Maryland, 1986.

2. Index to land use edge by type of land use and county, Maryland, 1986.

3. Area of timberland by county and ownership class, Maryland, 1986.

4. Area of timberland by forest type, forest-type group, and stand-size class, Maryland, 1986.

5. Area of timberland by county and stand-area class, Maryland, 1986.

6. Number of live nut-and fruit- producing trees on timberland by species and diameter class, Maryland, 1986.

7. Number of shrubs and saplings on timberland by stand-size class, type of stem, and mast type, Maryland, 1986.

8. Number of standing dead trees on timberland by species, condition class, and diameter class, Maryland, 1986.

9. Number of trees with observed cavities on timberland by species and condition class, Maryland, 1986.

10. Number of trees with observed cavities on timberland by species and presence of cavities, Maryland, 1986.

11. Number of seedlings, saplings, and shrubs on timberland by species and stand-size class, Maryland, 1986. forest-type group, Maryland, 1986.

13. Number of seedlings, saplings, and shrubs on timberland by species and browse-utilization class, Maryland, 1986.

\section{Central Unit}

14. Number of live nut- and fruit-producing trees on timberland by species and diameter class, Central Unit, Maryland, 1986.

15. Number of shrubs and saplings on timberland by stand-size class, type of stem, and mast type, Central Unit, Maryland, 1986.

16. Number of standing dead trees on timberland by species, condition, and diameter class, Central Unit, Maryland, 1986.

17. Number of trees with observed cavities on timberland by species and condition class, Central Unit, Maryland, 1986.

18. Number of trees with observed cavities on timberland by species and presence of cavities, Central Unit, Maryland, 1986.

19. Number of seedlings, saplings, and shrubs on timberland by species and stand-size class, Central Unit, Maryland, 1986.

20. Number of seedlings, saplings, and shrubs on timberland by species and forest-type group, Central Unit, Maryland, 1986.

21. Number of seedlings, saplings, and shrubs on timberland by species and browse-utilization class, Central Unit, Maryland, 1986. 
Lower Eastern Shore Unit

22. Number of live nut- and fruit- producing trees on timberland by species and diameter class, Lower Eastern Shore Unit, Maryland, 1986.

23. Number of shrubs and saplings on timberland by stand-size class, type of stem, and mast type, Lower Eastern Shore Unit, Maryland, 1986.

24. Number of standing dead trees on timberland by species, condition, and diameter class, Lower Eastern Shore Unit, Maryland, 1986.

25. Number of trees with observed cavities on timberland by species and condition class, Lower Eastern Shore Unit, Maryland, 1986.

26. Number of trees with observed cavities on timberland by species and presence of cavities, Lower Eastern Shore Unit, Maryland, 1986.

27. Number of seedlings, saplings, and shrubs on timberland by species and stand-size class, Lower Eastern Shore Unit, Maryland, 1986.

28. Number of seedlings, saplings, and shrubs on timberland by species and forest-type group, Lower Eastern Shore Unit, Maryland, 1986.

29. Number of seedlings, saplings, and shrubs on timberland by species and browse-utilization class, Lower Eastern Shore Unit, Maryland, 1986.

\section{Southern Unit}

30. Number of live nut- and fruit- producing trees on timberland by species and diameter class, Southern Unit, Maryland, 1986.

31. Number of shrubs and saplings on timberland by stand-size class, type of stem, and mast type, Southern Unit, Maryland, 1986.
32. Number of standing dead trees on timberland by species, condition class and diameter class, Southern Unit, Maryland, 1986.

33. Number of trees with observed cavities on timberland by species and condition class, Southern Unit, Maryland, 1986.

34. Number of trees with observed cavities on timberland by species and presence of cavities, Southern Unit, Maryland, 1986.

35. Number of seedlings, saplings, and shrubs on timberland by species and stand-size class, Southern Unit, Maryland, 1986.

36. Number of seedlings, saplings, and shrubs on timberland by species and forest-type group, Southern Unit, Maryland, 1986.

37. Number of seedlings, saplings, and shrubs on timberland by species and browse-utilization class, Southern Unit, Maryland, 1986.

\section{Western Unit}

38. Number of live nut- and fruit- producing trees on timberland by species and diameter class, Western Unit, Maryland, 1986.

39. Number of shrubs and saplings on timberland by stand-size class, type of stem, and mast type, Western Unit, Maryland, 1986.

40. Number of standing dead trees on timberland by species, condition class, and diameter class, Western Unit, Maryland, 1986.

41. Number of trees with observed cavities on timberland by species and condition class, Western Unit, Maryland, 1986.

42. Number of trees with observed cavities on timberland by species and presence 
of cavities, Western Unit, Maryland, 1986.

43. Number of seedlings, saplings, and shrubs on timberland by species and stand-size class, Western Unit, Maryland, 1986.

44. Number of seedlings, saplings, and shrubs on timberland by species and forest-type group, Western Unit, Maryland, 1986.

45. Number of seedlings, saplings, and shrubs on timberland by species and browse-utilization class, Western Unit, Maryland, 1986.

\section{County Tables}

46. Number of live nut- and fruit- producing tree on timberland by species and diameter class, Allegany County, Maryland, 1986.

47. Number of standing dead trees on timberland by species, condition class, and diameter class, Allegany County, Maryland, 1986.

48. Number of shrubs, seedlings, and saplings on timberland by species and browse-utilization class, Allegany County, Maryland, 1986.

49. Number of live nut- and fruit- producing trees on timberland by species and diameter class, Anne Arundel/Howard Counties, Maryland, 1986.

50. Number of standing dead trees on timberland by species, condition, and diameter class, Anne Arundel/Howard Counties, Maryland, 1986.

51. Number of shrubs, seedlings, and saplings on timberland by species and browse-utilization class, Anne Arundel/ Howard Counties, Maryland, 1986.

52. Number of live nut- and fruit- producing trees on timberland by species and di- ameter class, Baltimore County, Maryland, 1986.

53. Number of standing dead trees on timberland by species, condition class, and diameter class, Baltimore County, Maryland, 1986.

54. Number of shrubs, seedlings, and saplings on timberland by species and browse-utilization class, Baltimore County, Maryland, 1986.

55. Number of live nut- and fruit- producing trees on timberland by species and diameter class, Calvert County, Maryland, 1986.

56. Number of standing dead trees on timberland by species, condition class, and diameter class, Calvert County, Maryland, 1986.

57. Number of shrubs, seedlings, and saplings on timberland by species and browse-utilization class, Calvert County, Maryland, 1986.

58. Number of live nut- and fruit- producing trees on timberland by species and diameter class, Caroline/Talbot Counties, Maryland, 1986.

59. Number of standing dead trees on timberland by species, condition class, and diameter class, Caroline/Talbot Counties, Mayland, 1986.

60. Number of shrubs, seedlings, and saplings on timberland by species and browse-utilization class, Caroline/ Talbot Counties, Maryland, 1986.

61. Number of live nut- and fruit-producing trees on timberland by species and diameter class, Carroll County, Maryland, 1986.

62. Number of standing dead trees on timberland by species, condition class, and diameter class, Carroll County, Maryland, 1986. 
63. Number of shrubs, seedlings, and saplings on timberland by species and browse-utilization class, Carroll County, Maryland, 1986.

64. Number of live nut- and fruit- producing trees on timberland by species and diameter class, Cecil/Harford Counties, Maryland, 1986.

65. Number of standing dead trees on timberland by species, condition class, and diameter class, Cecil/Harford Counties, Maryland, 1986.

66. Number of shrubs, seedlings, and saplings on timberland by species and browse-utilization class, Cecil/Harford Counties, Maryland, 1986.

67. Number of live nut- and fruit- producing trees on timberland by species and diameter class, Charles County, Maryland, 1986.

68. Number of standing dead trees on timberland by species, condition class, and diameter class, Charles County, Maryland, 1986.

69. Number of shrubs, seedlings, and saplings on timberland by species and browse-utilization class, Charles County, Maryland, 1986.

70. Number of live nut- and fruit- producing trees on timberland by species and diameter class, Dorchester County, Maryland, 1986.

71. Number of standing dead trees on timberland by species, condition, and diameter class, Dorchester County, Maryland, 1986.

72. Number of shrubs, seedlings, and saplings on timberland by species and browse-utilization class, Dorchester County, Maryland, 1986.

73. Number of live nut- and fruit- producing trees on timberland by species and di- ameter class, Frederick County, Maryland, 1986.

74. Number of standing dead trees on timberland by species, condition class, and diameter class, Frederick County, Maryland, 1986.

75. Number of shrubs, seedlings, and saplings on timberland by species and browse-utilization class, Frederick County, Maryland, 1986.

76. Number of live nut- and fruit- producing trees on timberland by species and diameter class, Garrett County, Maryland, 1986.

77. Number of standing dead trees on timberland by species, condition class, and diameter class, Garrett County, Maryland, 1986.

78. Number of shrubs, seedlings, and saplings on timberland by species and browse-utilization class, Garrett County, Maryland, 1986.

79. Number of live nut- and fruit- producing trees on timberland by species and diameter class, Kent/Queen Annes Counties, Maryland, 1986.

80. Number of standing dead trees on timberland by species, condition class, and diameter class, Kent/Queen Annes Counties, Maryland, 1986.

81. Number of shrubs, seedlings, and saplings on timberland by species and browse-utilization class, Kent/Queen Annes Counties, Maryland, 1986.

82. Number of live nut- and fruit- producing trees on timberland by species and diameter class, Montgomery/Prince Georges Counties, Maryland, 1986.

83. Number of standing dead trees on timberland by species, condition class, and diameter class, Montgomery/ Prince Georges Counties, Maryland, 1986. 
84. Number of shrubs, seedlings, and saplings on timberland by species and browse-utilization class, Montgomery/ Prince Georges Counties, Maryland, 1986.

85. Number of live nut- and fruit- producing trees on timberland by species and diameter class, St. Marys County, Maryland, 1986.

86. Number of standing dead trees on timberland by species, condition class, and diameter class, St. Marys County, Maryland, 1986.

87. Number of shrubs, seedlings, and saplings on timberland by species and browse-utilization class, St. Marys County, Maryland, 1986.

88. Number of live nut- and fruit- producing trees on timberland by species and diameter class, Somerset County, Maryland, 1986.

89. Number of standing dead trees on timberland by species, condition class, and diameter class, Somerset County, Maryland, 1986.

90. Number of shrubs, seedlings, and saplings on timberland by species and browse-utilization class, Somerset County, Maryland, 1986.

91. Number of live nut- and fruit-producing trees on timberland by species and diameter class, Washington County, Maryland, 1986.

92. Number of standing dead trees on timberland by species, condition class, and diameter class, Washington County, Maryland, 1986.

93. Number of shrubs, seedlings, and saplings on timberland by species and browse-utilization class, Washington County, Maryland, 1986.

94. Number of live nut- and fruit- producing trees on timberland by species and di- ameter class, Wicomico County, Maryland, 1986.

95. Number of standing dead trees on timberland by species, condition class, and diameter class, Wicomico County, Maryland, 1986.

96. Number of shrubs, seedlings, and saplings on timberland by species and browse-utilization class, Wicomico County, Maryland, 1986.

97. Number of live nut- and fruit producing trees on timberland by species and diameter class, Worcester County, Maryland, 1986.

98. Number of standing dead trees on timberland by species, condition class, and diameter class, Worcester County, Maryland, 1986.

99. Number of shrubs, seedlings, and saplings on timberland by species and browse-utilization class, Worcester County, Maryland, 1986.

\section{Delaware Tables}

\section{State Tables}

100. Land area by county and land class, Delaware, 1986.

101. Index to land use edge by type of land use and county, Delaware, 1986.

102. Area of timberland by county and ownership class, Delaware, 1986.

103. Area of timberland by forest type, forest-type group, and stand-size class, Delaware, 1986.

104. Area of timberland by county and stand-area class, Delaware, 1986.

105. Number of live nut- and fruit- producing trees on timberland by species and diameter class, Delaware, 1986. 
106. Number of shrubs and saplings on timberland by stand-size class, type of stem, and mast type, Delaware, 1986.

107. Number of standing dead trees on timberland by species, condition class, and diameter class, Delaware, 1986.

108. Number of trees with observed cavities on timberland by species and condition class, Delaware, 1986.

109. Number of trees with observed cavities on timberland by species and presence of cavities, Delaware, 1986.

110. Number of seedlings, saplings, and shrubs on timberland by species and stand-size class, Delaware, 1986.

111. Number of seedlings, saplings, and shrubs on timberland by species and forest-type group, Delaware, 1986.

112. Number of seedlings, saplings, and shrubs on timberland by species and browse-utilization class, Delaware, 1986.

\section{County Tables}

113. Number of live nut- and fruit- producing trees on timberland by species and diameter class, Kent/New Castle Counties, Delaware, 1986.

114. Number of standing dead trees on timberland by species, condition class, and diameter class, Kent/New Castle Counties, Delaware, 1986.

115. Number of shrubs, seedlings, and saplings on timberland by species and browse-utilization class, Kent/New Castle Counties, Delaware, 1986.

116. Number of live nut- and fruit- producing trees on timberland by species and diameter class, Sussex County, Delaware, 1986.

117. Number of standing dead trees on timberland by species, condition class, and diameter class, Sussex County, Delaware, 1986.

118. Number of shrubs, seedlings, and saplings on timberland by species, browse-preference class, and browseutilization class, Sussex County, Delaware, 1986. 


\section{MARYLAND \\ STATE TABLES}

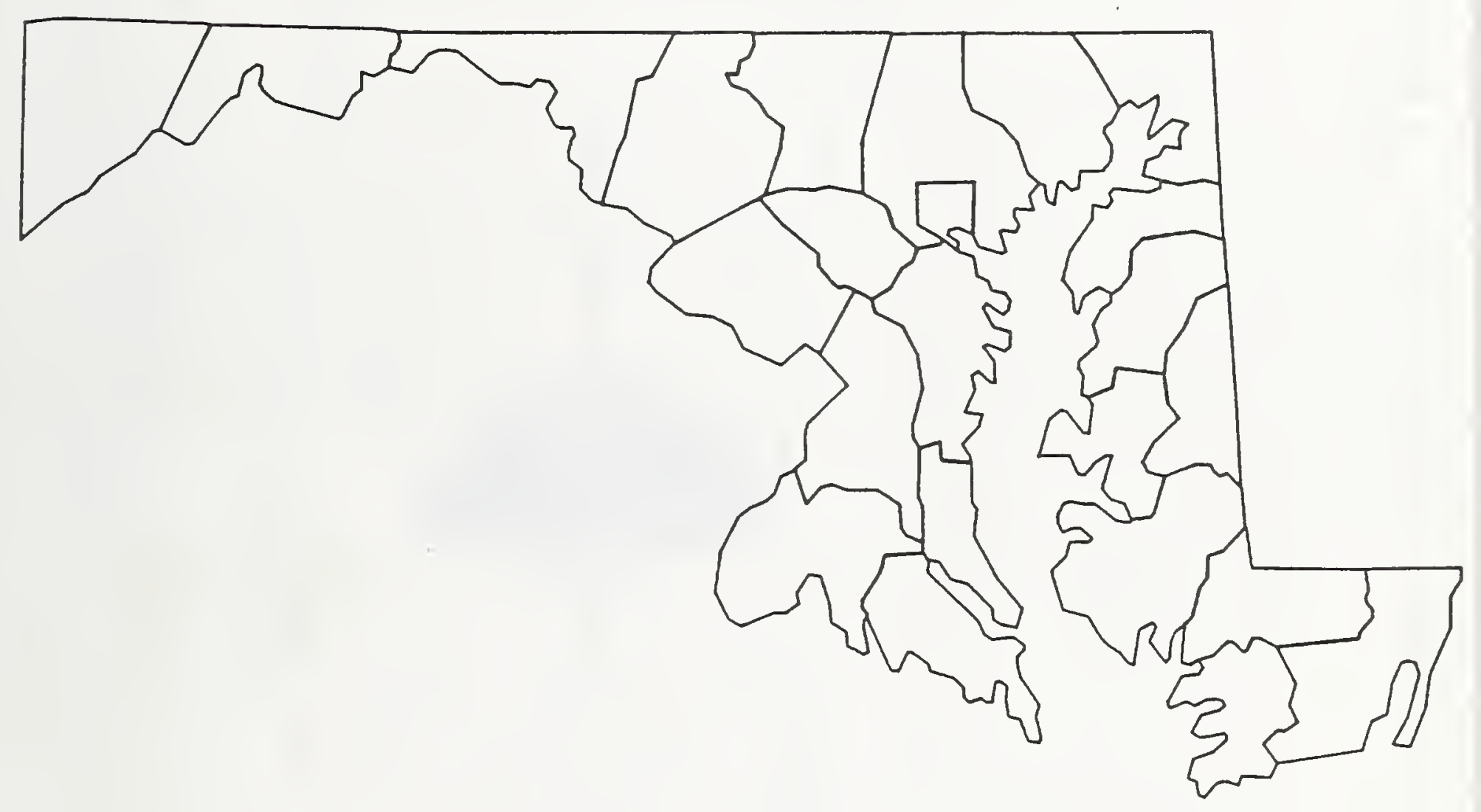



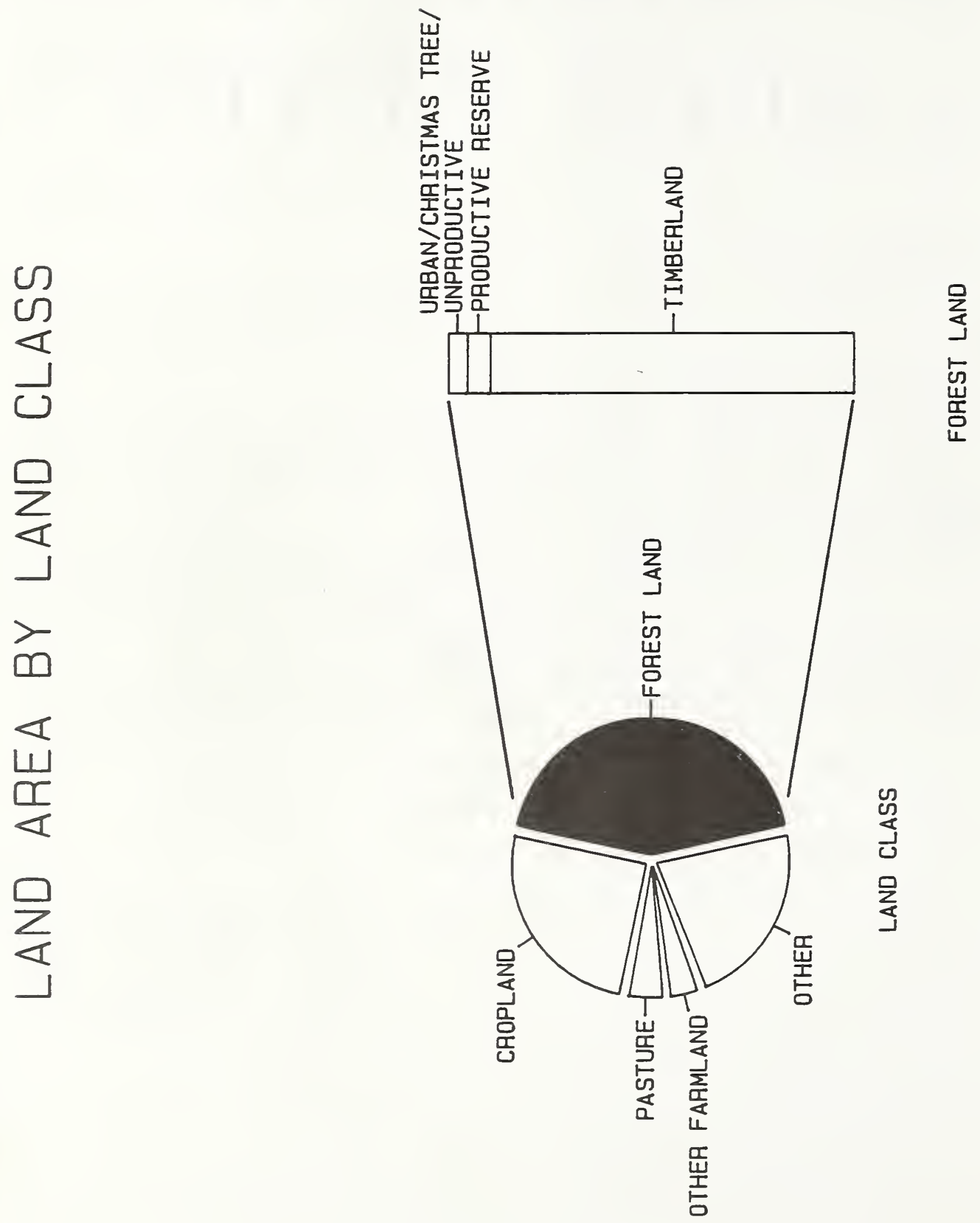


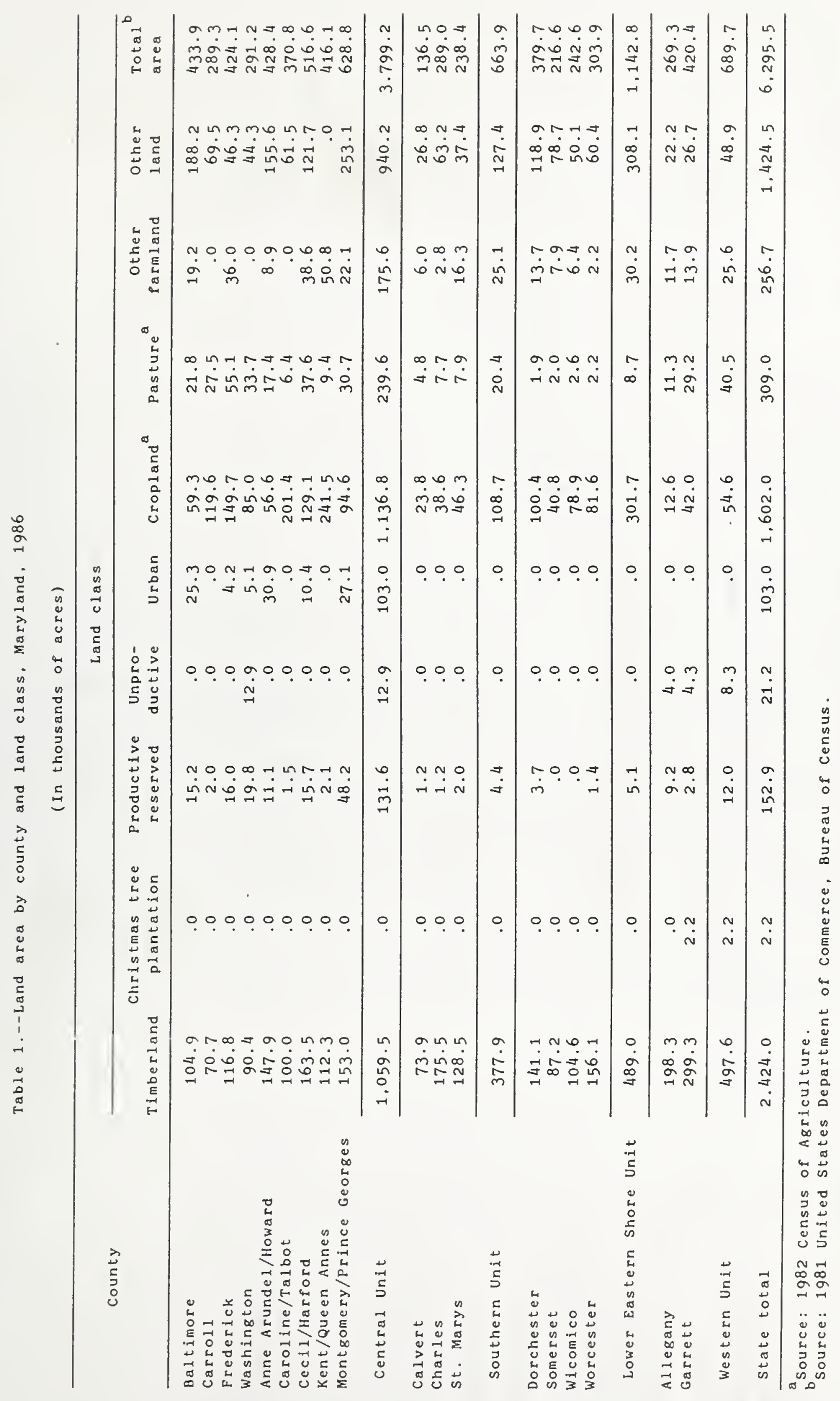




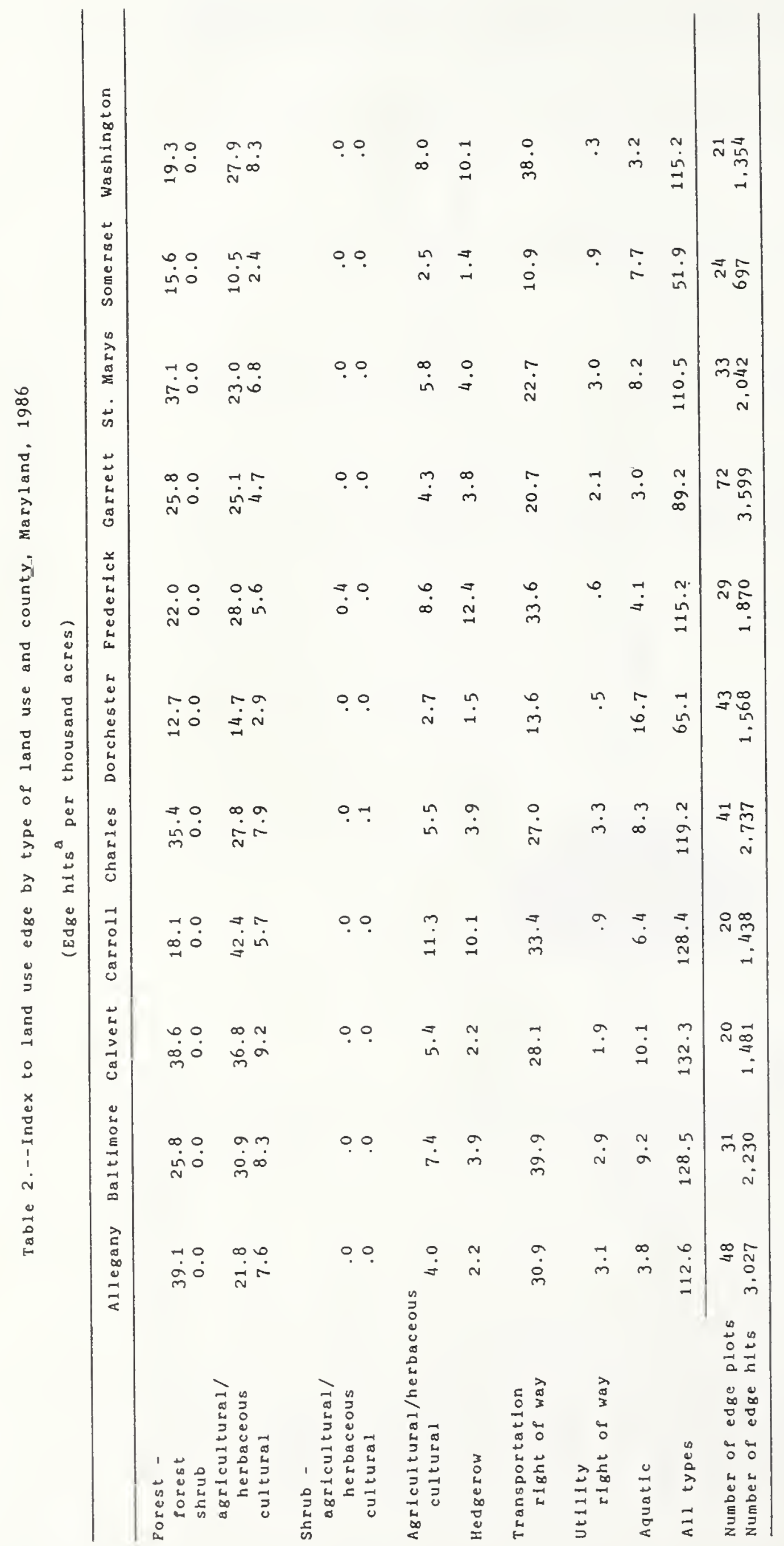




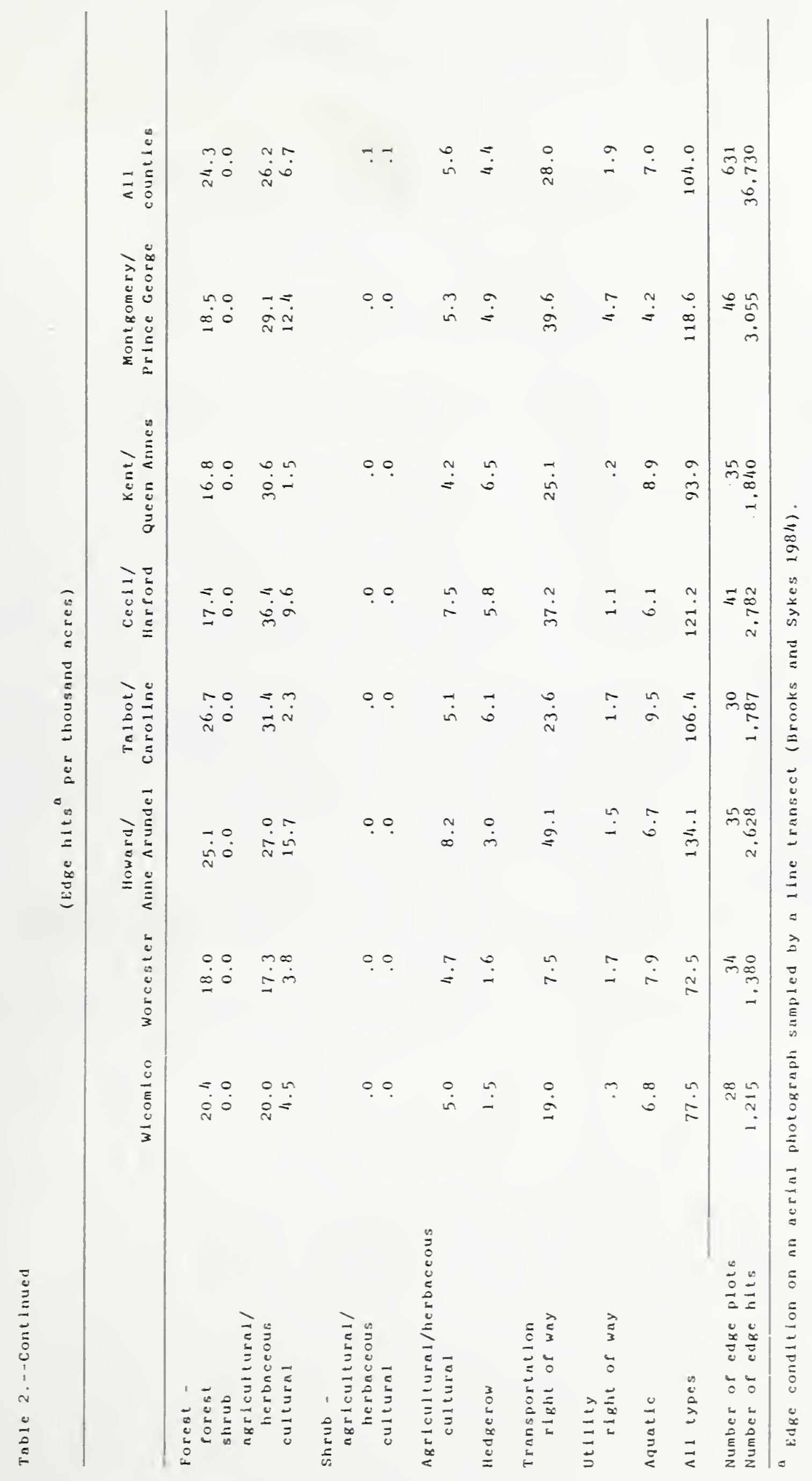




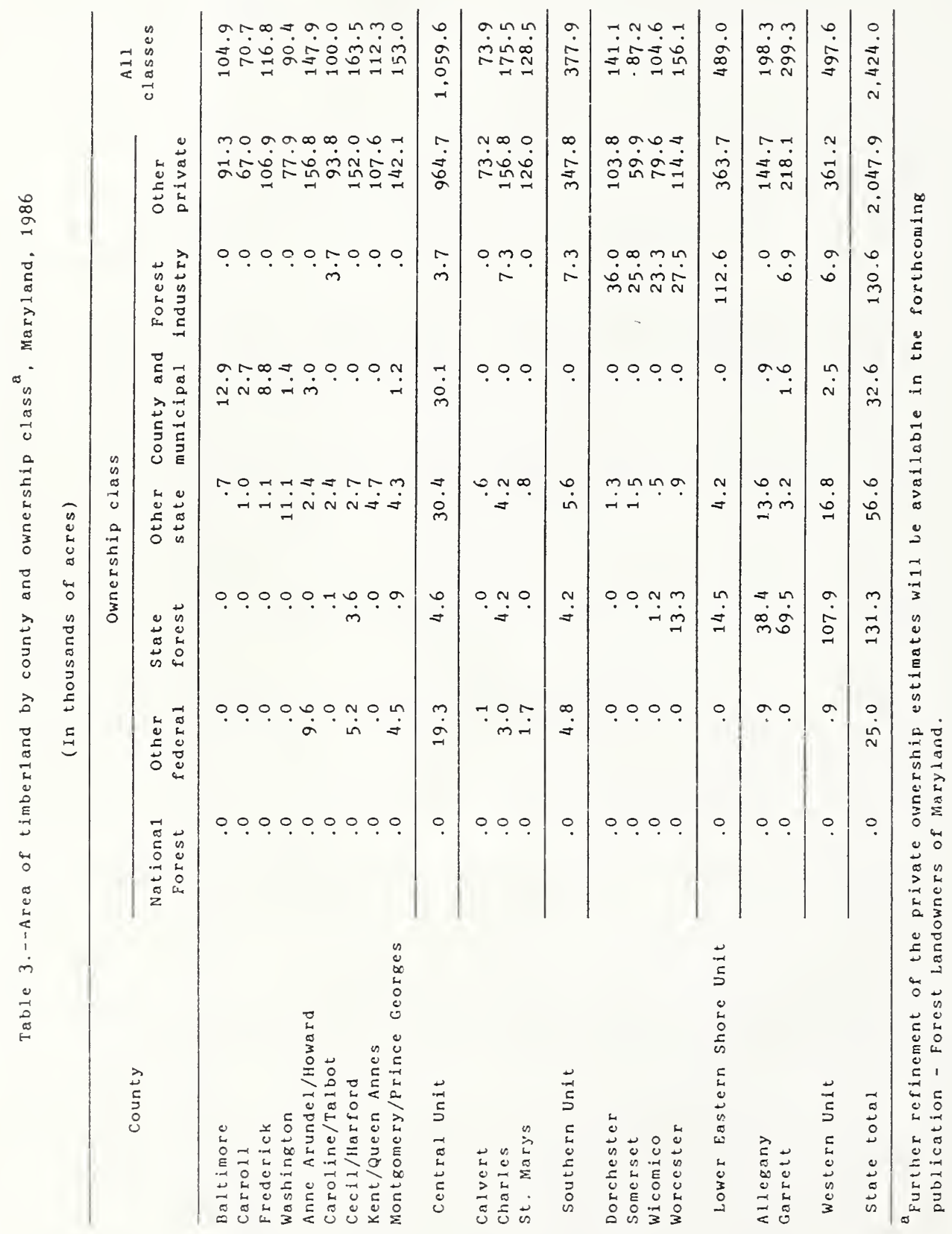




\section{PERCENT TIMBERLAND BY FOREST TYPE GROUP}

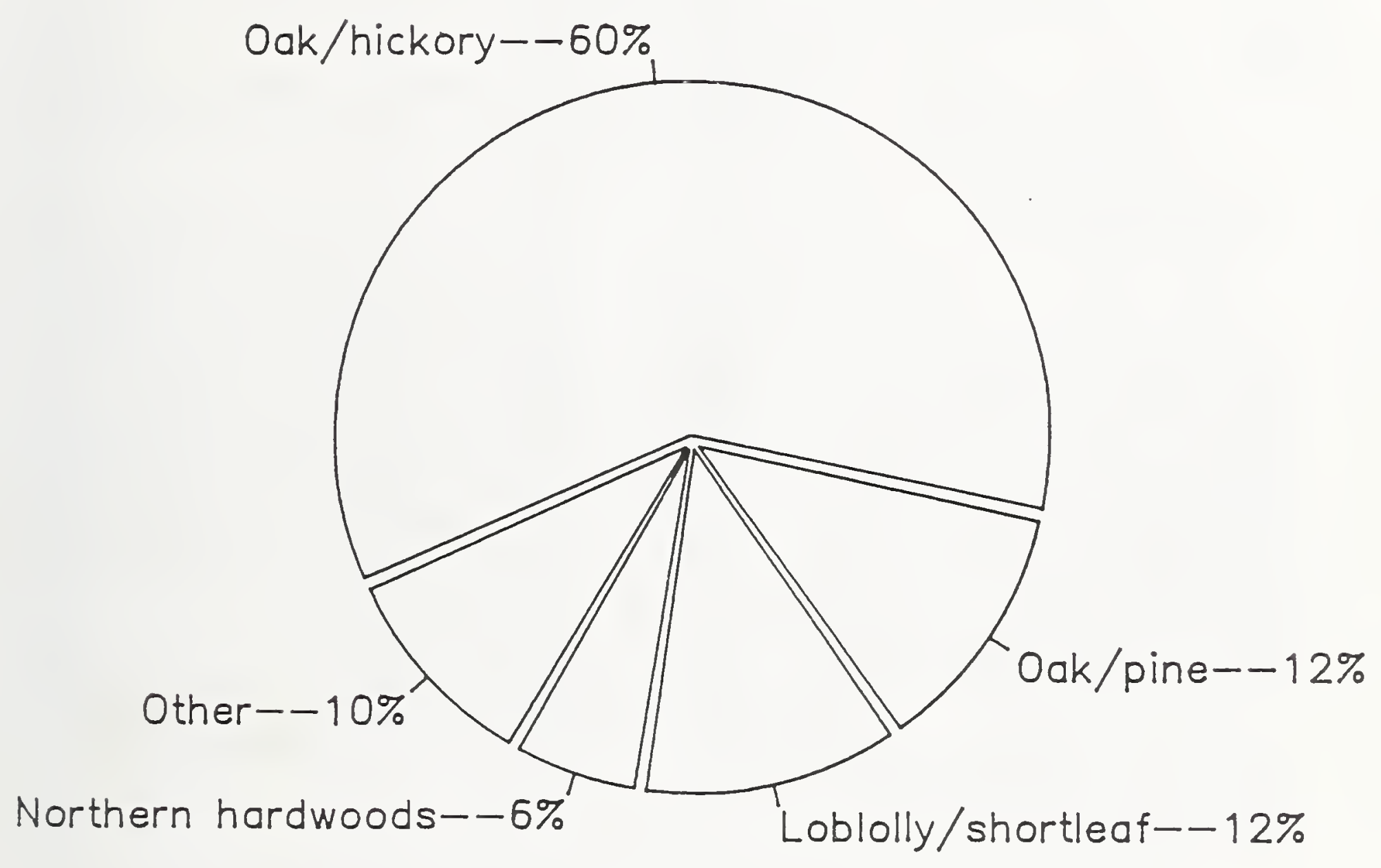


Table 4.--Area op timberland by forest type, forest-type group, and stand-size class. Maryland, 1986

(In thousands of acres)

\begin{tabular}{|c|c|c|c|c|c|}
\hline \multirow{2}{*}{ Forest type } & \multicolumn{4}{|c|}{ Stand-slze class } & \multirow{2}{*}{$\begin{array}{c}\text { A } 11 \\
\text { classes }\end{array}$} \\
\hline & Sawt $1 \mathrm{mber}$ & Poletimber & $\begin{array}{l}\text { Sapling and } \\
\text { seedling }\end{array}$ & Nonstocked & \\
\hline Red pine & $3 \cdot 1$ & 14.6 & .0 & .0 & 17.8 \\
\hline White plne & 6.4 & $4 \cdot 5$ & .0 & .0 & 10.9 \\
\hline Hemlock & 6.9 & .0 & $3 \cdot 9$ & .0 & 10.8 \\
\hline scotch pline & .0 & $3 \cdot 1$ & $10 \cdot 5$ & .0 & 13.6 \\
\hline White/red pine group & 16.4 & $22 \cdot 3$ & 14.4 & .0 & 53.1 \\
\hline Lob1ol1y pine & 128.1 & $41 \cdot 3$ & $17 \cdot 5$ & .0 & 186.8 \\
\hline virginia pine & $65 \cdot 5$ & 22.6 & $22.0=$ & .0 & 110.1 \\
\hline Loblolly/shortleaf group & 193.6 & 63.9 & 39.4 & .0 & 296.9 \\
\hline Wh. pine/no. red oak/wh. ash & $9 \cdot 5$ & 2.4 & .0 & .0 & 11.9 \\
\hline VIrginla pine/oak & 78.6 & 5.2 & 11.7 & .0 & $95 \cdot 5$ \\
\hline Loblo1ly pine/hardwood & 122.1 & 17.6 & 24.7 & .0 & 164.4 \\
\hline other oak/pine & 5.4 & 4.7 & .0 & .0 & 10.2 \\
\hline Oak/pIne group & 215.6 & 29.9 & 36.4 & .0 & 281.9 \\
\hline Post, black, or bear oak & $17 \cdot 0$ & 6.0 & $4 \cdot 5$ & .0 & 27.6 \\
\hline Chestnut oak & $59 \cdot 9$ & 3.4 & .0 & .0 & $63 \cdot 3$ \\
\hline White oak/red oak/hlckory & 83.2 & 24.8 & 9.5 & .0 & $117 \cdot 6$ \\
\hline White oak & 31.8 & $34 \cdot 5$ & 6.8 & .0 & 73.1 \\
\hline Northern red oak & 31.0 & .0 & .0 & .0 & 31.0 \\
\hline Y. poplar/wh. oak/no. red oak & 66.4 & 12.4 & .0 & .0 & 78.8 \\
\hline Black locust & .0 & .0 & 9.8 & .0 & 9.8 \\
\hline Sweetgum/ye110w-pop1ar & 66.6 & 16.8 & $15 \cdot 1$ & .0 & 98.5 \\
\hline Ye11 ow-pop1ar & 116.4 & .0 & $7 \cdot 7$ & .0 & 124.1 \\
\hline Hawthorn reverting fleld & $3 \cdot 2$ & $4 \cdot 5$ & .0 & .0 & $7 \cdot 7$ \\
\hline Scarlet oak & 8.2 & .0 & 4.1 & .0 & 12.2 \\
\hline Sassafras/persimmon & .0 & .0 & 2.8 & .0 & 2.8 \\
\hline Red maple/central hardwoods & 32.6 & 19.7 & 4.2 & .0 & 56.5 \\
\hline Mixed central hardwoods & 570.9 & 160.6 & 19.8 & .0 & $751 \cdot 3$ \\
\hline Oak/hickory group & $1,087 \cdot 3$ & 282.8 & 84.4 & .0 & $\therefore .454 .4$ \\
\hline Swamp chstnt oak/cherrybark oak & 30.1 & $10 \cdot 5$ & .0 & .0 & 40.6 \\
\hline Sweetgum/nuttall oak/w11low oak & 40.1 & 14.0 & 10.0 & .0 & 64.1 \\
\hline Ba1d cypress/white tupelo & 3.0 & .0 & .0 & .0 & 3.0 \\
\hline Sweetbay/swamp cupelo/red maple & $7 \cdot 1$ & $2 \cdot 9$ & $2 \cdot 5$ & .0 & $12 \cdot 5$ \\
\hline Oak/gum/cypress group & $80 \cdot 3$ & $27 \cdot 4$ & $12 \cdot 5$ & .0 & 120.2 \\
\hline Black ash/Amer. elm/red maple & $29 \cdot 5$ & .0 & 1.9 & .0 & 31.4 \\
\hline Red maple(lowland) & 8.8 & .0 & 2.2 & .0 & 10.9 \\
\hline Red maple (upland) & .0 & .0 & 3.8 & .0 & 3.8 \\
\hline River birch/sycamore & 19.4 & 4.1 & .0 & .0 & $23 \cdot 5$ \\
\hline Willow & .0 & 2.1 & .0 & .0 & 2.1 \\
\hline Sycamore/pecan/American elm & 4.6 & .0 & .0 & .0 & 4.6 \\
\hline
\end{tabular}


Table 4.--Continued

(In thousands of acres)

\begin{tabular}{|c|c|c|c|c|c|}
\hline \multirow{2}{*}{ Porest type } & \multicolumn{4}{|c|}{ stand-size class } & \multirow{2}{*}{$\begin{array}{c}\text { All } \\
\text { classes }\end{array}$} \\
\hline & Sawt $1 \mathrm{mber}$ & Poletimber & $\begin{array}{l}\text { Sapling and } \\
\text { seedling }\end{array}$ & Nonstocked & \\
\hline American e $1 \mathrm{~m} / \mathrm{green}$ ash & $7 \cdot 4$ & .0 & .0 & .0 & $7 \cdot 4$ \\
\hline Elm/ash/red maple group & 69.6 & 6.2 & $7 \cdot 9$ & .0 & $83 \cdot 7$ \\
\hline sugar maple/beech/yellow blrch & 30.5 & .0 & $7 \cdot 3$ & .0 & 37.8 \\
\hline Black cherry & 6.7 & 14.2 & 4.6 & .0 & $25 \cdot 6$ \\
\hline Red maple/northern hardwoods & 3.1 & 6.8 & 4.2 & .0 & 14.1 \\
\hline Pin cherry/reverting pleld & .0 & $5 \cdot 1$ & $17 \cdot 5$ & .0 & 22.6 \\
\hline Mixed northern hardwoods & 20.7 & $10 \cdot 3$ & 2.6 & .0 & 33.6 \\
\hline Northern hardwoods group & 61.0 & $36 \cdot 5$ & $36 \cdot 3$ & .0 & 133.8 \\
\hline All forest types & 1.723 .8 & 468.9 & $231 \cdot 3$ & .0 & 2.424 .0 \\
\hline
\end{tabular}


Table 5.--Area of timberland by county and stand-area class. Maryland. 1986

(In thousands of acres)

\begin{tabular}{|c|c|c|c|c|c|c|c|}
\hline \multirow{2}{*}{ County } & \multicolumn{6}{|c|}{ Stand-area class } & \multirow{2}{*}{$\begin{array}{c}\text { All } \\
\text { classes }\end{array}$} \\
\hline & $1-9$ & $10-19$ & $20-49$ & $50-99$ & $100-499$ & $500+$ & \\
\hline Baltimore & 12.0 & 49.4 & $33 \cdot 3$ & $10 \cdot 3$ & .0 & .0 & 104.9 \\
\hline Carroll & $21 \cdot 7$ & 36.1 & 12.8 & .0 & .0 & .0 & 70.7 \\
\hline Frederick & $27 \cdot 7$ & $32 \cdot 7$ & 28.7 & 14.2 & $13 \cdot 5$ & .0 & 116.8 \\
\hline Washington & 12.6 & 18.6 & 40.8 & $11 \cdot 3$ & $7 \cdot 2$ & .0 & 90.4 \\
\hline Anne Arundel/Howard & 87.4 & 37.8 & 22.7 & .0 & .0 & .0 & 147.9 \\
\hline Caroline/Talbot & 31.2 & 24.2 & 30.9 & 13.8 & .0 & .0 & 100.0 \\
\hline Cecil/Harford & $52 \cdot 7$ & 40.2 & $58 \cdot 3$ & 12.4 & .0 & .0 & 163.5 \\
\hline Kent/Queen Annes & 36.4 & 36.8 & 24.9 & 5.9 & $7 \cdot 2$ & .0 & $112 \cdot 3$ \\
\hline Montgomery/Prince Georges & 82.6 & 38.8 & 19.4 & 8.4 & 3.8 & .0 & 153.0 \\
\hline Central Unit & $364 \cdot 3$ & 314.5 & 271.7 & $77 \cdot 4$ & 31.7 & .0 & 1.059 .6 \\
\hline Calvert & 40.5 & $27 \cdot 5$ & 5.8 & .0 & .0 & .0 & $73 \cdot 9$ \\
\hline Charles & 64.5 & 48.7 & 50.6 & 10.0 & .0 & 1.6 & $175 \cdot 5$ \\
\hline St. Marys & 41.7 & $37 \cdot 2$ & 46.7 & 2.9 & .0 & .0 & 128.5 \\
\hline Southern Unit & 146.7 & $113 \cdot 5$ & 103.2 & 12.9 & .0 & 1.6 & 377.9 \\
\hline Dorchester & 10.5 & 31.8 & $52 \cdot 5$ & 30.5 & $15 \cdot 9$ & .0 & 141.1 \\
\hline Somerset & 15.4 & 11.4 & 40.4 & $15 \cdot 3$ & 4.6 & .0 & 87.2 \\
\hline Wicomico & 9.7 & 27.8 & 36.0 & 28.5 & $2 \cdot 5$ & .0 & 104.6 \\
\hline Worcester & $35 \cdot 1$ & 44.3 & 26.0 & $18 \cdot 7$ & $27 \cdot 9$ & 4.2 & 156.1 \\
\hline Lower Eastern Shore Unit & 70.8 & 115.2 & 154.9 & 93.0 & 50.9 & 4.2 & 489.0 \\
\hline Allegany & 72.4 & $59 \cdot 7$ & 40.5 & 18.9 & 6.9 & .0 & 198.3 \\
\hline Garrett & 87.8 & 82.8 & 62.6 & 47.6 & $18 \cdot 5$ & .0 & 299.3 \\
\hline Western Unit & 160.2 & 142.5 & 103.0 & 66.5 & $25 \cdot 3$ & .0 & 497.6 \\
\hline State total & 741.9 & 685.7 & 632.8 & 249.8 & 108.0 & 5.8 & 2.424 .0 \\
\hline
\end{tabular}




\section{TOP TEN NUT- AND FRUIT-PRODUCING TREES Millions of trees}






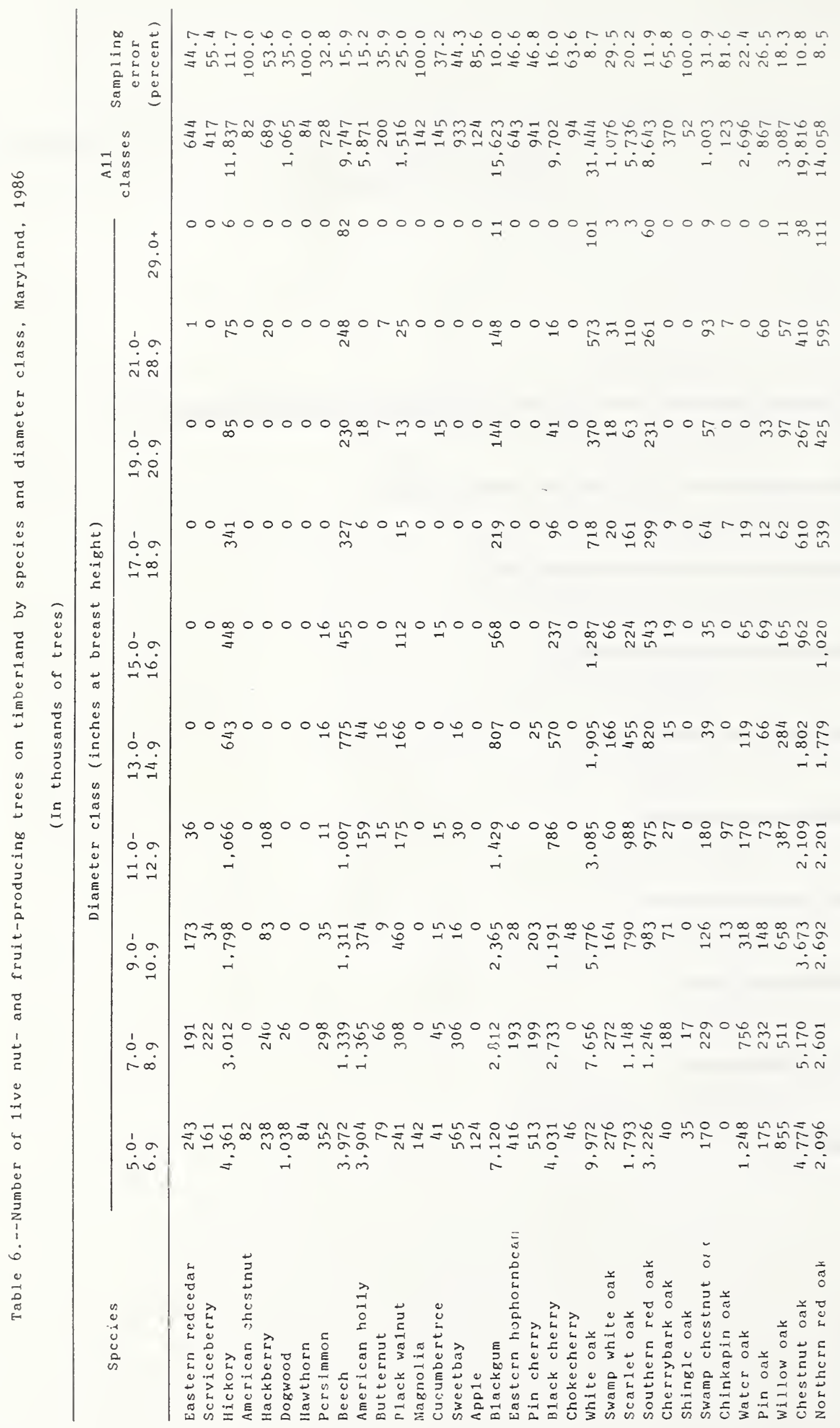




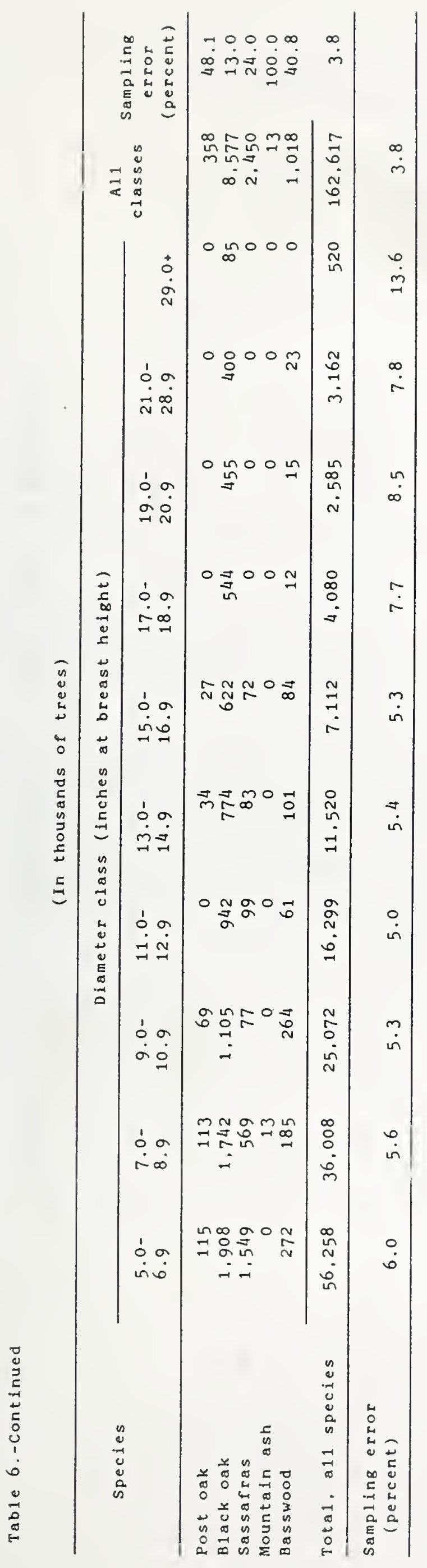


Table 7.--Number of shrubs and saplings on timberland by stand-size class. type of stem, and mast type. Mary1and, 1986

(In thousands of stems)

\begin{tabular}{|c|c|c|c|c|c|}
\hline \multirow{2}{*}{$\begin{array}{l}\text { Stand-size class } \\
\text { and type of stem }\end{array}$} & \multicolumn{4}{|c|}{ Mast type } & \multirow{2}{*}{$\begin{array}{l}\text { Total } \\
\text { stens }\end{array}$} \\
\hline & Nuts & seeds & Berries & species & \\
\hline \multicolumn{6}{|l|}{ Sawtimber: } \\
\hline Shrubs & 0 & 198.776 & $1,001.688$ & 387.188 & 1.587 .652 \\
\hline Saplings & 85.461 & 260.943 & 216.938 & 1.990 & 565.332 \\
\hline Total sawtimber & 85.461 & 459.719 & $1,218,626$ & 389.178 & 2.152 .984 \\
\hline \multicolumn{6}{|l|}{ Poletimber: } \\
\hline Shrubs & 2.198 & 50.765 & 294.510 & 97.949 & 445,423 \\
\hline Saplings & 37.919 & 136,778 & 52,968 & 0 & 227.665 \\
\hline Tota1 poletimber & 40.117 & 187.543 & 347.478 & 97.949 & 673.089 \\
\hline \multicolumn{6}{|l|}{ Sapling/seed 1 ing: } \\
\hline Shrubs & 0 & 13.933 & 172.838 & 56.880 & 243.651 \\
\hline Saplings & 12.759 & 93.041 & 23.372 & 1.604 & 130.775 \\
\hline Total sap1ing/seed1ing & 12,759 & 106.975 & 196.209 & 58.484 & 374.427 \\
\hline Tota1, a11 classes & 138.337 & 754.237 & 1.762 .314 & 545,611 & 3.200 .499 \\
\hline
\end{tabular}




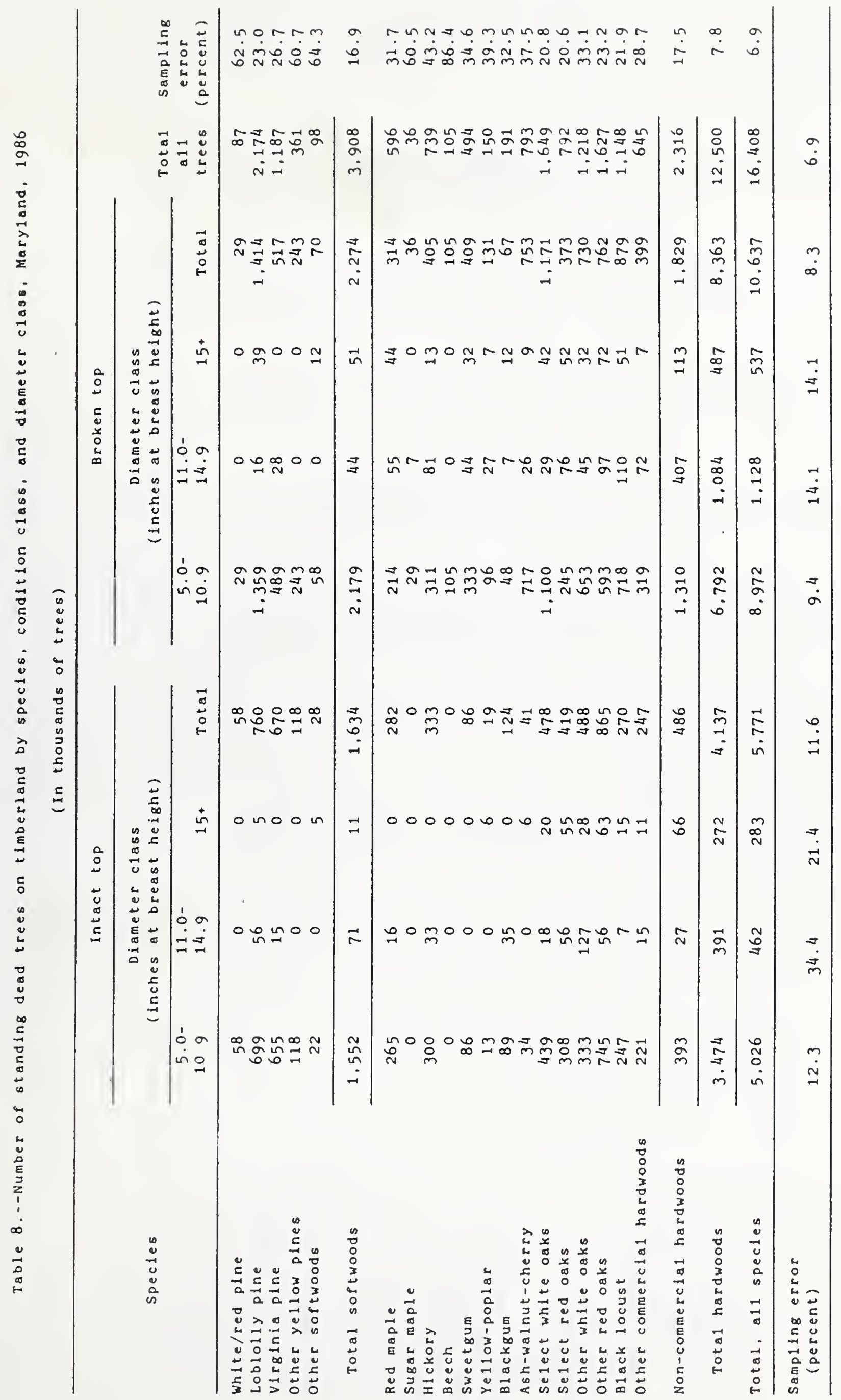




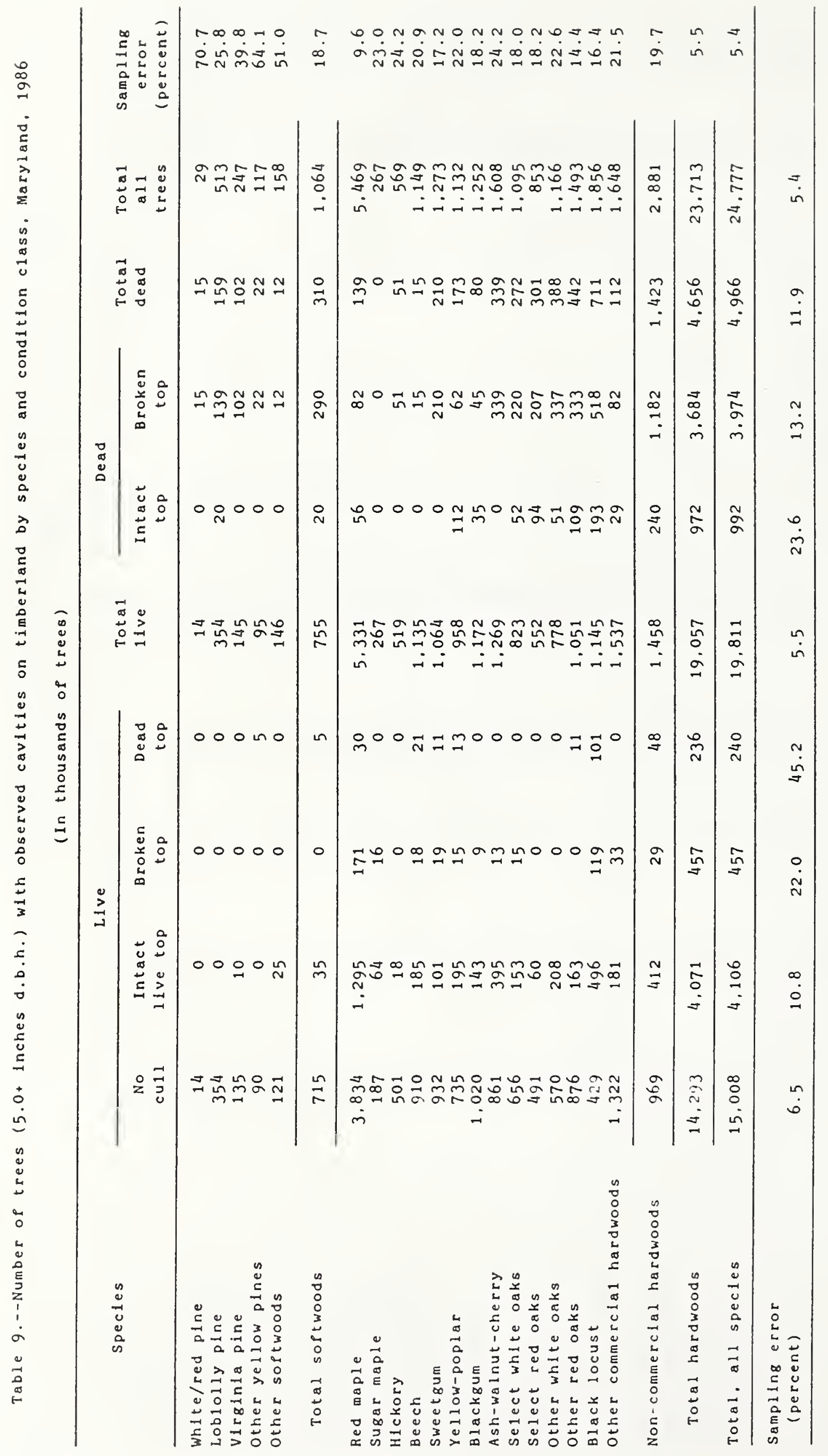




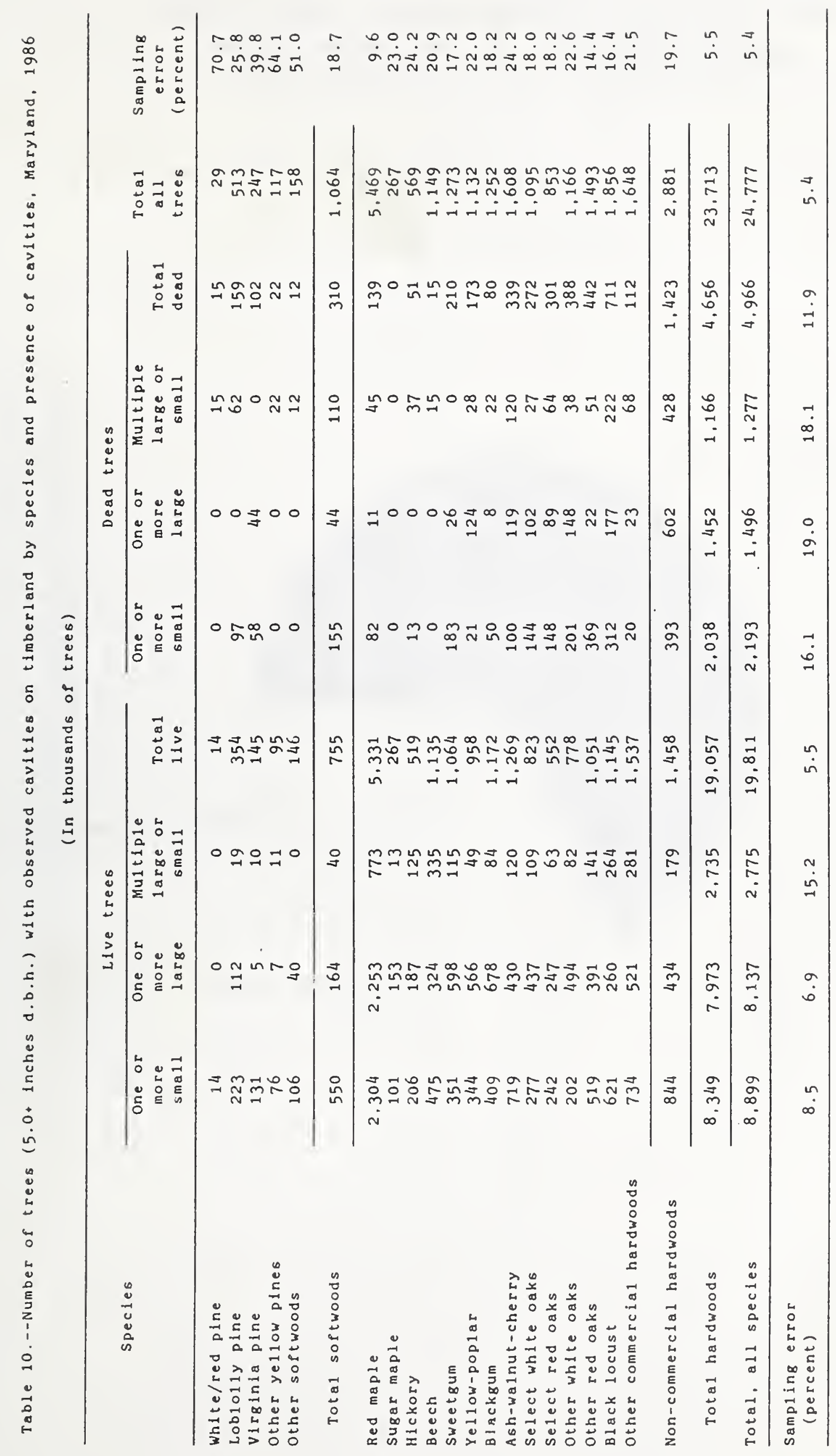




\section{PERCENT OF SEEDLINGS, SAPLINGS, AND SHRUBS BY STAND-SIZE}

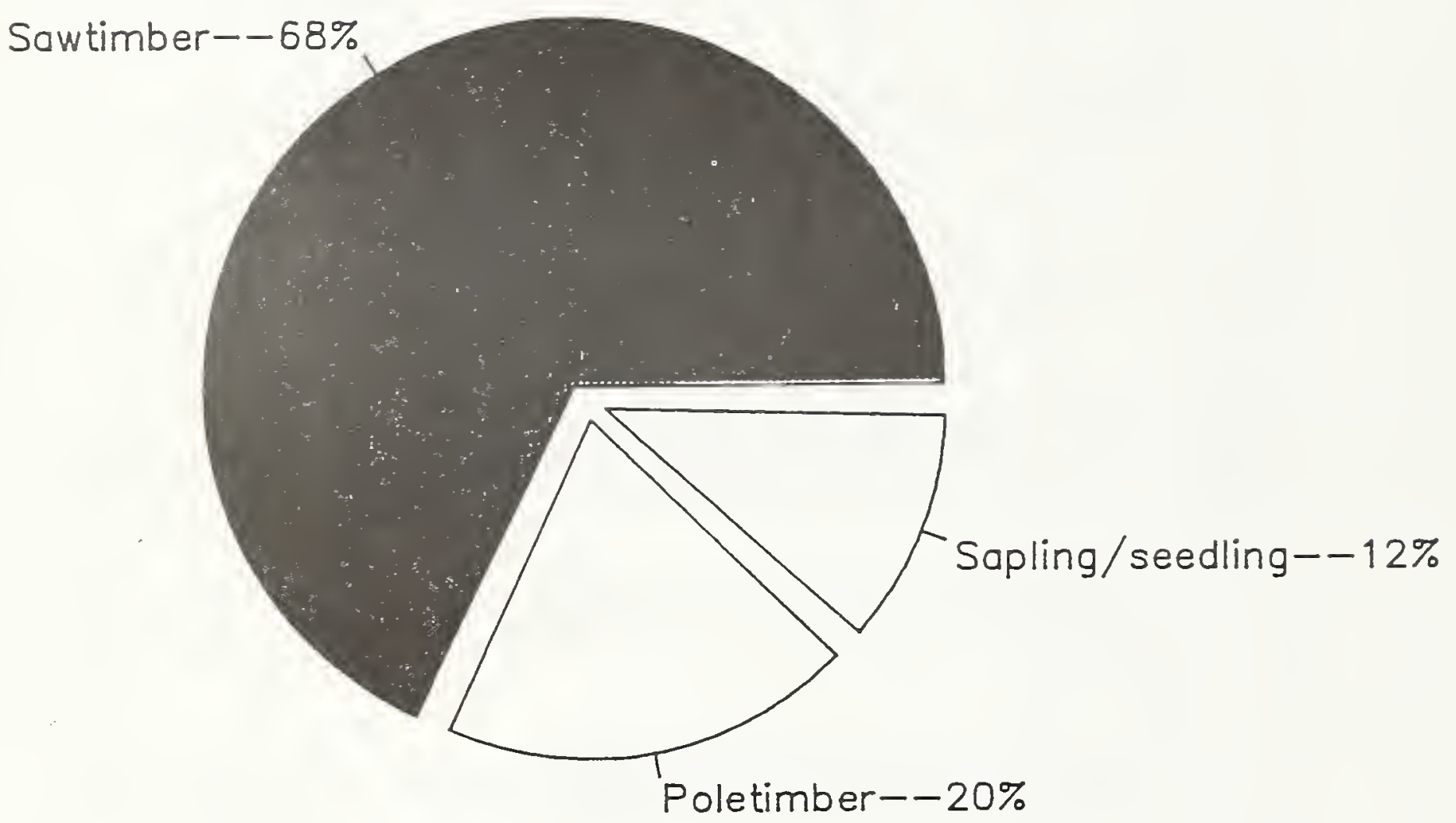


Table 11,--Number of seedlings, saplings, and shrubs on timberland by species and stand-size class, Maryland, 1986

\begin{tabular}{|c|c|c|c|c|c|c|}
\hline \multirow{2}{*}{ Species } & \multicolumn{4}{|c|}{ Stand-size class } & \multirow{2}{*}{$\begin{array}{c}\text { All } \\
\text { classes }\end{array}$} & \multirow{2}{*}{$\begin{array}{c}\text { Sampling } \\
\text { error } \\
\text { (percent) }\end{array}$} \\
\hline & \multicolumn{4}{|c|}{$\begin{array}{l}\text { Sapling and } \\
\text { Sanstocked }\end{array}$} & & \\
\hline Loblolly pine & 74 & 43 & 67 & 0 & 183 & 20.4 \\
\hline Other pine species & 32 & 11 & 30 & 0 & 73 & $17 \cdot 9$ \\
\hline Other softwoods & 20 & 7 & 6 & 0 & 33 & $21 \cdot 1$ \\
\hline Total coniferous species & 127 & 61 & 102 & 0 & 290 & $13 \cdot 9$ \\
\hline Réd maple & 921 & 329 & 336 & 0 & 1.586 & 8.0 \\
\hline Sugar maple & 99 & 33 & 14 & 0 & 146 & 22.4 \\
\hline Other maple species & 112 & 20 & 12 & 0 & 144 & $23 \cdot 7$ \\
\hline Alder spectes & 17 & 4 & 0 & 0 & 22 & 31.9 \\
\hline Serviceberry & 132 & 73 & 14 & 0 & 219 & 17.4 \\
\hline Azalea species & 53 & 13 & 3 & 0 & 70 & 14.7 \\
\hline Common pawpaw & 245 & 0 & 0 & 0 & 245 & 24.5 \\
\hline Blrch spectes & 111 & 15 & 17 & 0 & 143 & $27 \cdot 1$ \\
\hline Hickory species & 113 & 40 & 8 & 0 & 161 & 12.4 \\
\hline Dogwood species & 355 & 82 & 24 & 0 & 461 & 12.8 \\
\hline American beech & 167 & 25 & 1 & 0 & 193 & $15 \cdot 3$ \\
\hline Ash species & 159 & 46 & 43 & 0 & 248 & $17 \cdot 9$ \\
\hline Huckleberry & 10 & 15 & 4 & 0 & 59 & 18.8 \\
\hline Witch-hazel & 40 & 12 & 3 & 0 & 55 & $14 \cdot 5$ \\
\hline American holly & 242 & 82 & 34 & 0 & 357 & 10.1 \\
\hline Laurel species & 67 & 20 & 6 & 0 & 93 & $15 \cdot 1$ \\
\hline Common spicebush & 121 & 11 & 2 & 0 & 135 & $13 \cdot 5$ \\
\hline Sweetgum & 412 & 117 & 111 & 0 & 641 & 9.2 \\
\hline Yellow-poplar & 178 & 26 & 41 & 0 & 245 & 24.4 \\
\hline Magnolia specles & 99 & 11 & 3 & 0 & 113 & 26.4 \\
\hline Tupelo species & 365 & 123 & 50 & 0 & 538 & 11.8 \\
\hline Black cherry & 482 & 172 & 46 & 0 & 701 & $13 \cdot 5$ \\
\hline Other cherry species & 24 & 10 & 17 & 0 & 51 & $25 \cdot 6$ \\
\hline White oak & 166 & 55 & 37 & 0 & 259 & 16.1 \\
\hline Chestnut oak & 139 & 14 & 10 & 0 & 163 & $21 \cdot 7$ \\
\hline Other white oaks & 30 & 5 & 2 & 0 & 37 & 39.0 \\
\hline Northern red oak & 139 & 29 & 11 & 0 & 179 & $15 \cdot 3$ \\
\hline Other black oaks & 252 & 87 & 44 & 0 & 383 & 12.4 \\
\hline Rhododendron species & 18 & 1 & 0 & 0 & 19 & 39.4 \\
\hline Rose species & 66 & 17 & 19 & 0 & 102 & $21 \cdot 5$ \\
\hline Rubus species & 219 & 73 & 89 & 0 & 382 & $7 \cdot 2$ \\
\hline Sassafras & 291 & 98 & 57 & 0 & 446 & $13 \cdot 3$ \\
\hline Blueberry & 340 & 114 & 36 & 0 & 489 & $5 \cdot 7$ \\
\hline Elm species & 68 & 12 & 2 & 0 & 82 & 24.9 \\
\hline Maple-leaved viburnum & 61 & 7 & 3 & 0 & 72 & 15.2 \\
\hline Arrowwood & 72 & 20 & 4 & 0 & 95 & 1.3 .1 \\
\hline Other viburnum species & 45 & 13 & 1 & 0 & 59 & 16.2 \\
\hline Other deciduous species & 343 & 129 & 110 & 0 & 583 & $9 \cdot 7$ \\
\hline Total deciduous species & 6.809 & 1.955 & 1.216 & 0 & 9.980 & 3.6 \\
\hline Unknown species & 413 & 97 & 48 & 0 & 558 & $6 \cdot 3$ \\
\hline Total, all species & 7.349 & 2.113 & 1,366 & 0 & 10.828 & $3 \cdot 5$ \\
\hline Sampling error & & & & & & \\
\hline$(p e r c e n t)$ & 4.7 & 11.2 & 14.7 & .0 & $3 \cdot 5$ & \\
\hline
\end{tabular}




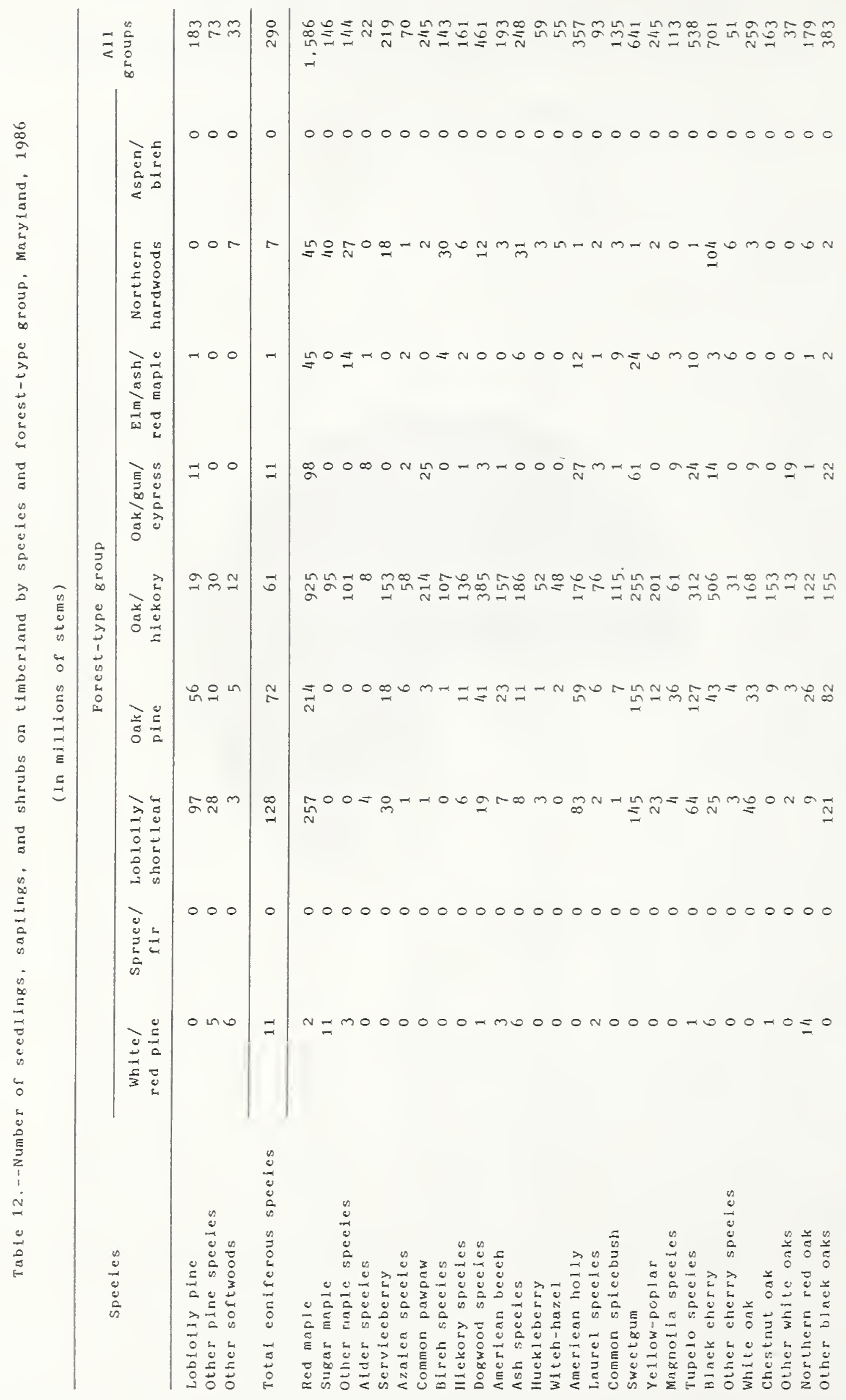







Table 13.--Number of seedlings, saplings, and shrubs on timberland by species and browse-utilization class, Maryland, 1986

\begin{tabular}{|c|c|c|c|c|c|c|}
\hline \multirow{2}{*}{ Species } & \multicolumn{4}{|c|}{ Browse-ut111zation class } & \multirow{2}{*}{$\begin{array}{c}\text { All } \\
\text { classes }\end{array}$} & \multirow{2}{*}{$\begin{array}{c}\text { Sampling } \\
\text { error } \\
\text { (percent) }\end{array}$} \\
\hline & None & Light & Moderate & Heavy & & \\
\hline Loblol1y pine & 183 & 0 & 0 & 1 & 183 & 20.4 \\
\hline Other pine species & 73 & 0 & 0 & 0 & 73 & $17 \cdot 9$ \\
\hline Other softwoods & 33 & 0 & 0 & 0 & 33 & 21.1 \\
\hline Total conlferous species & 289 & 0 & 0 & 1 & 290 & 13.9 \\
\hline Red maple & 1,379 & 190 & 16 & 1 & 1.586 & 8.0 \\
\hline Sugar maple & 105 & 35 & 6 & 0 & 146 & 22.4 \\
\hline Other maple species & 132 & 10 & 0 & 2 & 144 & $23 \cdot 7$ \\
\hline Alder species & 19 & 2 & 0 & 0 & 22 & 31.9 \\
\hline Serviceberry & 195 & 21 & 2 & 1 & 219 & 17.4 \\
\hline Azalea species & 63 & 5 & 2 & 0 & 70 & 14.7 \\
\hline Common pawpaw & 221 & 24 & 0 & 0 & 245 & 24.5 \\
\hline Birch species & 131 & 5 & 7 & 0 & 143 & $27 \cdot 1$ \\
\hline Hickory species & 148 & 10 & 3 & 0 & 161 & 12.4 \\
\hline Dogwood species & 429 & 17 & 15 & 0 & 461 & 12.8 \\
\hline American beech & 176 & 15 & 2 & 1 & 193 & $15 \cdot 3$ \\
\hline Ash species & 212 & 29 & 7 & 0 & 248 & $17 \cdot 9$ \\
\hline Huckleberry & 52 & 7 & 0 & 0 & 59 & 18.8 \\
\hline Witch-hazel & 46 & 6 & 2 & 1 & 55 & 14.5 \\
\hline Amerlcan holly & 343 & 4 & 10 & 1 & 357 & 10.1 \\
\hline Laurel species & 91 & 1 & 1 & 0 & 93 & 15.1 \\
\hline Common spicebush & 122 & 8 & 3 & 2 & 135 & $13 \cdot 5$ \\
\hline Sweet Bum & 601 & 36 & 0 & 4 & 641 & 9.2 \\
\hline Yellow-poplar & 234 & 9 & 2 & 0 & 245 & 24.4 \\
\hline Magnolia species & 102 & 10 & 0 & 0 & 113 & 26.4 \\
\hline Tupelo spectes & 468 & 50 & 14 & 6 & 538 & 11.8 \\
\hline Black cherry & 538 & 151 & 12 & 0 & 701 & 13.5 \\
\hline Other cherry species & 48 & 1 & 0 & 2 & 51 & 25.6 \\
\hline White oak & 226 & 13 & 4 & 16 & 259 & 16.1 \\
\hline Chestnut oak & 143 & 12 & 7 & 1 & 163 & 21.7 \\
\hline Other white oaks & 37 & 0 & 0 & 0 & 37 & 39.0 \\
\hline Northern red oak & 151 & 22 & 6 & 0 & 179 & $15 \cdot 3$ \\
\hline Other black oaks & 355 & 23 & 5 & 0 & 383 & 12.4 \\
\hline Rhododendron species & 19 & 0 & 0 & 0 & 19 & 39.4 \\
\hline Rose species & 97 & 5 & 1 & 0 & 102 & $21 \cdot 5$ \\
\hline Rubus species & 359 & 20 & 2 & 0 & 382 & $7 \cdot 2$ \\
\hline Sassafras & 359 & 71 & 16 & 0 & 446 & $13 \cdot 3$ \\
\hline Blueberry & 383 & 88 & 14 & 5 & 489 & 5.7 \\
\hline E1m species & 66 & 13 & 3 & 0 & 82 & 24.9 \\
\hline Maple-1caved viburnum & 55 & 14 & 3 & 1 & 72 & 15.2 \\
\hline Arrowwood & 85 & 7 & 3 & 0 & 95 & 13.1 \\
\hline Other viburnum species & 48 & 8 & 3 & 0 & 59 & 16.2 \\
\hline Other deciduous species & 476 & 99 & 4 & 4 & 583 & 9.7 \\
\hline Total deciduous species & 8.719 & 1,040 & 174 & 47 & 9.980 & 3.6 \\
\hline Unknown species & 442 & 91 & 20 & 6 & 558 & 6.3 \\
\hline Total, all species & $9.1: 50$ & 1.131 & 194 & 54 & 10.828 & $3 \cdot 5$ \\
\hline $\begin{array}{l}\text { Sampling error } \\
\text { (percent) }\end{array}$ & 3.6 & 9.4 & 12.7 & 36.0 & $3 \cdot 5$ & \\
\hline
\end{tabular}




$$
\begin{gathered}
\text { MARYLAND } \\
\text { CENTRAL UNIT } \\
\text { TABLES }
\end{gathered}
$$

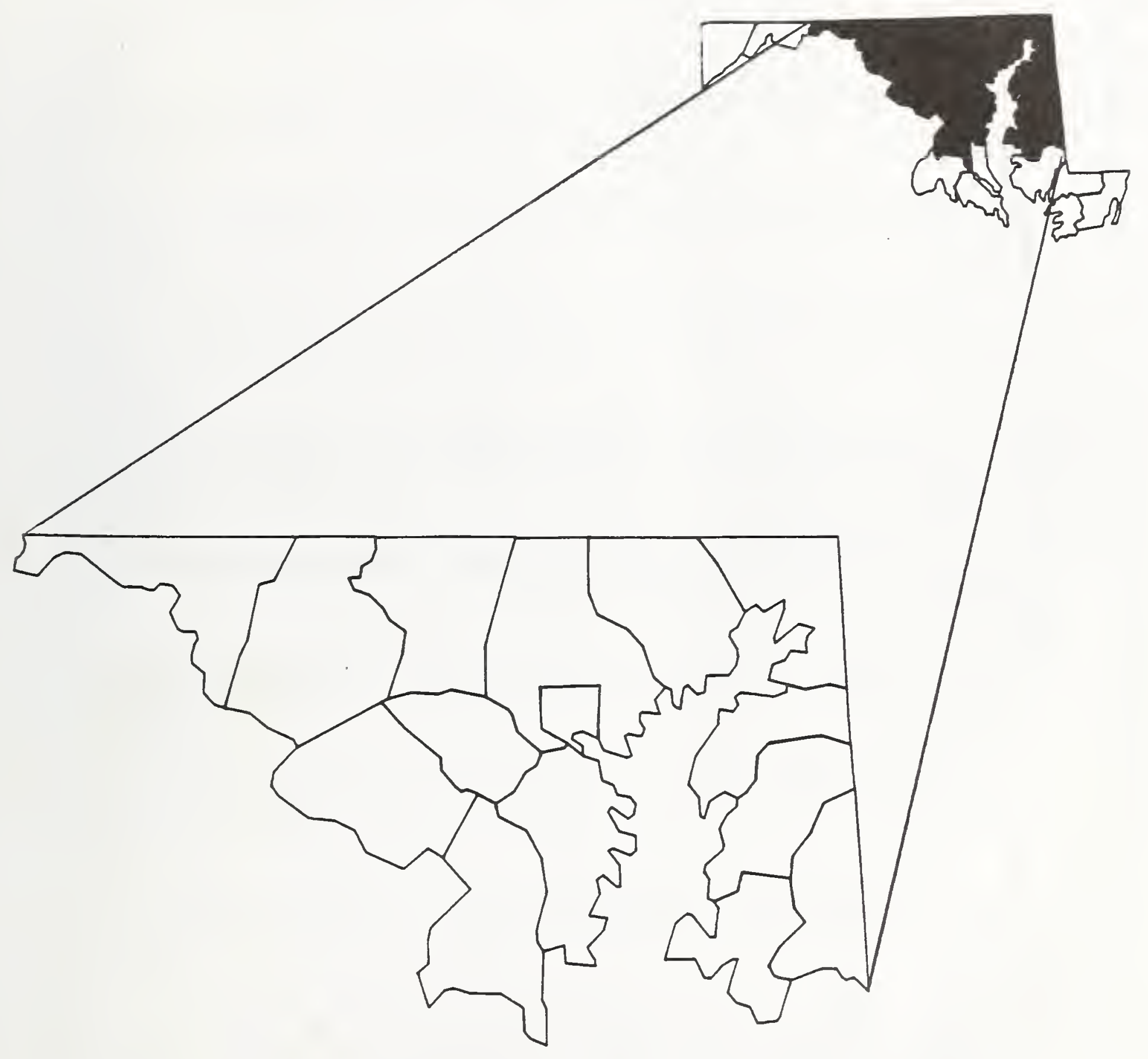





ம்

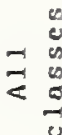



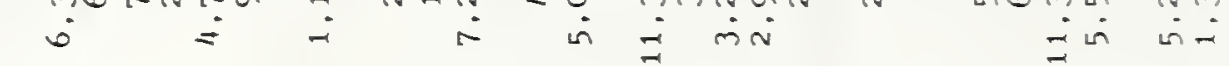

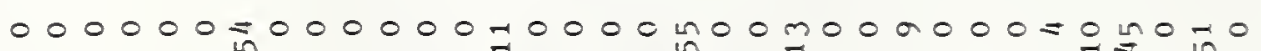
0
$\overline{2}$
$m$
in

10

-ON

$\vec{\sim} \stackrel{\infty}{\sim}$

ba



ㅇำ

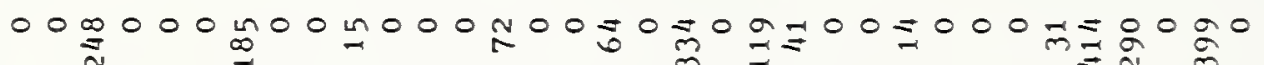

$\begin{array}{lll}1 & 0 \\ 0 & 0 & 0\end{array}$

$\operatorname{tin}_{-\infty}^{\infty}$



i.

N

.

$-$

○ ${\underset{N}{N}}_{\text {N }}$ o

$0_{0}^{1} a$

$\sim \equiv$

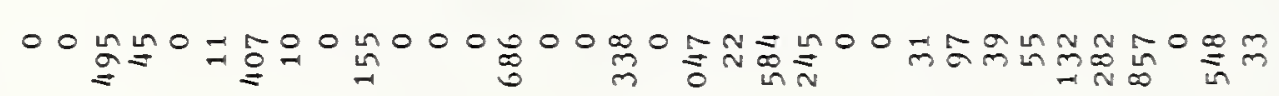

$\stackrel{i}{\because} \underset{\sim}{\sim}$

(n)

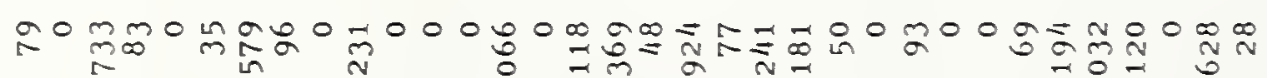

$\begin{array}{ll}1 & 0 \\ 0 & 0\end{array}$

(1)

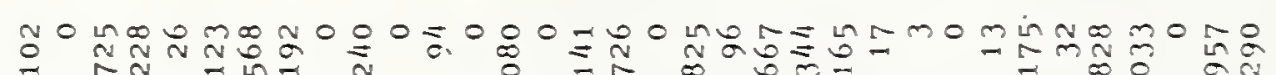

la

$\therefore \infty$

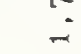

(19)

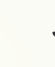

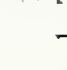

i

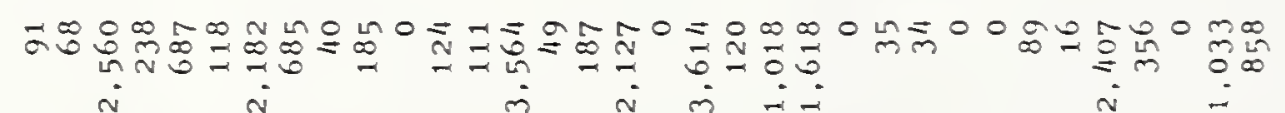

1
0
nin

$$
\text { N }
$$

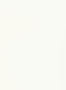


Tab1e 15.--Number of shrubs and sapjings on timberland by stand-size class, type of stem, and mast type, Central unit, Marylard, 1986

(In thousands of stems)

\begin{tabular}{|c|c|c|c|c|c|}
\hline \multirow{2}{*}{$\begin{array}{l}\text { Stand-size class } \\
\text { and type of stem }\end{array}$} & \multicolumn{4}{|c|}{ Mast type } & \multirow{2}{*}{$\begin{array}{l}\text { Total } \\
\text { stems }\end{array}$} \\
\hline & Nuts & seeds & Berries & species & \\
\hline \multicolumn{6}{|l|}{ Sawt Imber: } \\
\hline Shrúbs & 0 & 83.100 & 563.493 & 134,139 & 780.732 \\
\hline Saplings & 34.361 & 98.574 & 108,301 & 1.245 & 242,480 \\
\hline Total sawtimber & 34.361 & 181.674 & 671.794 & 135.383 & 1.023 .212 \\
\hline \multicolumn{6}{|l|}{ Poletimber: } \\
\hline Shrubs & 0 & 16.247 & 132.965 & 28,586 & 177.799 \\
\hline Saplings & 15.043 & 40.018 & 14.829 & 0 & 69.890 \\
\hline Total poletimber & 15.043 & 56.265 & 147.794 & 28,586 & 247.689 \\
\hline \multicolumn{6}{|l|}{ Sapling/seedling: } \\
\hline Shrubs & 0 & 3,811 & 76,400 & 5.590 & 85,801 \\
\hline Saplings & 865 & 31.718 & 6.562 & 0 & 39.145 \\
\hline Total sapling/seedling & 865 & 35.529 & 82,962 & 5.590 & 124.945 \\
\hline Total, all classes & 50,268 & 273.468 & 902.550 & 169.500 & 1.395 .845 \\
\hline
\end{tabular}




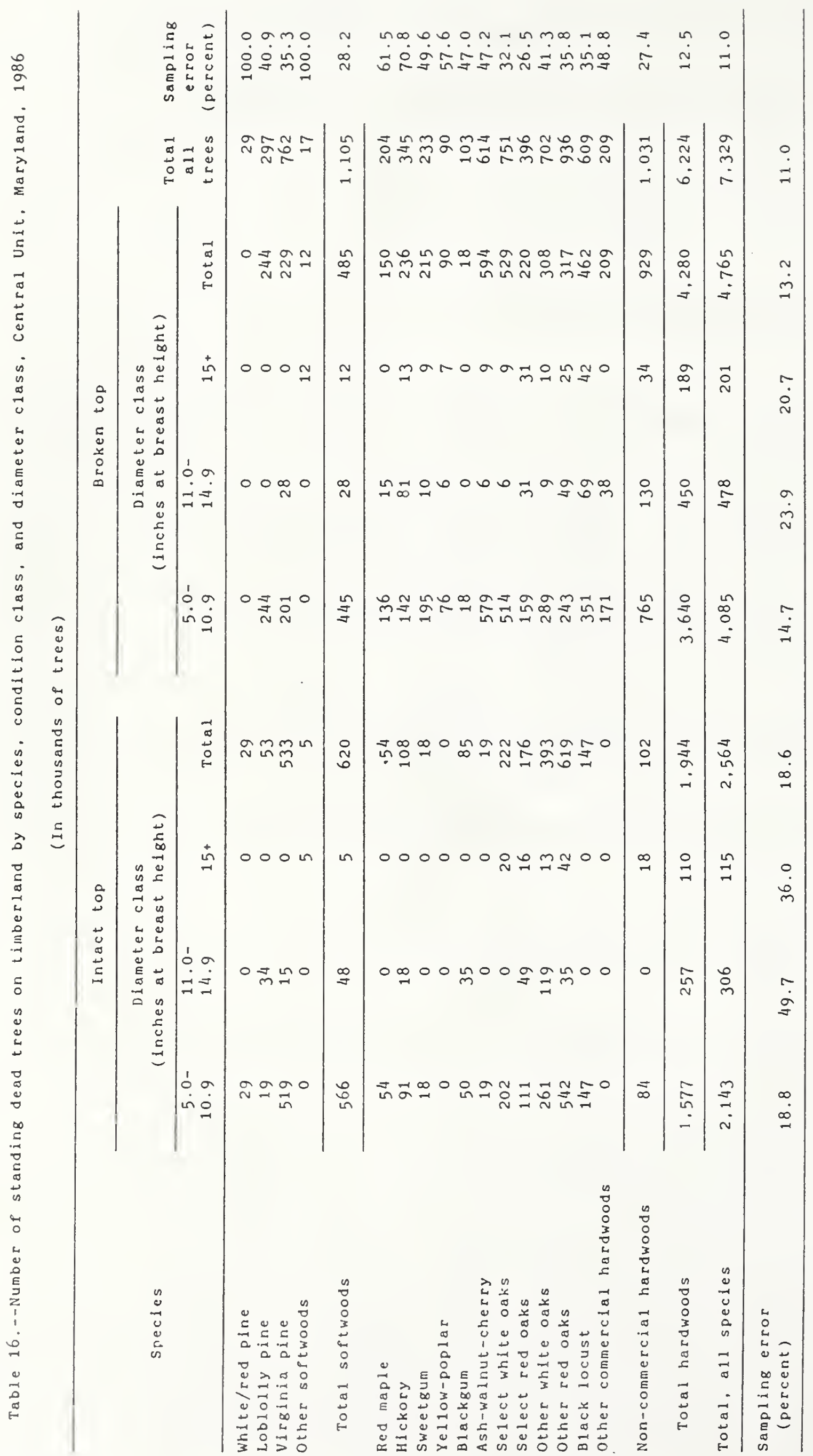




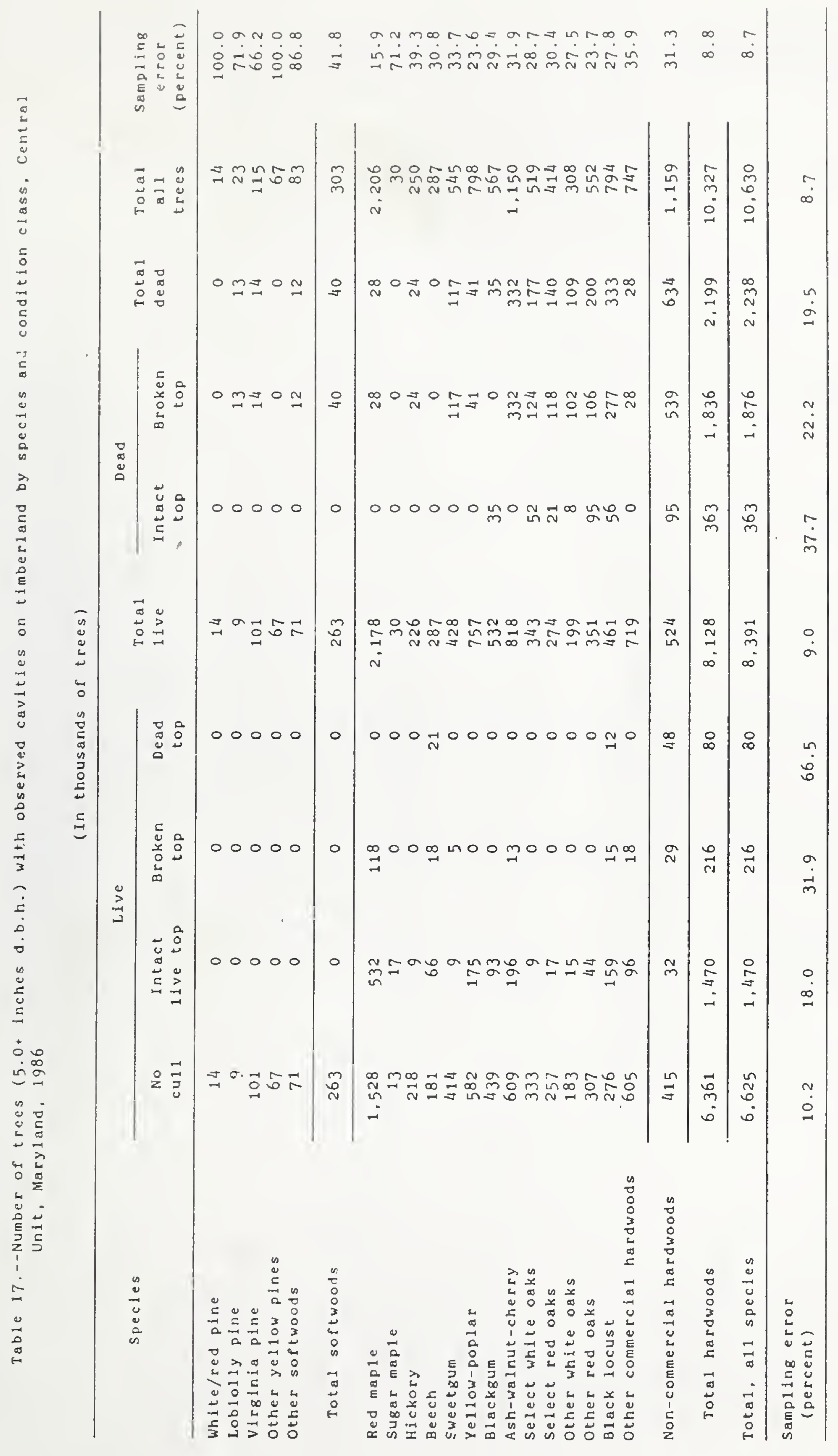




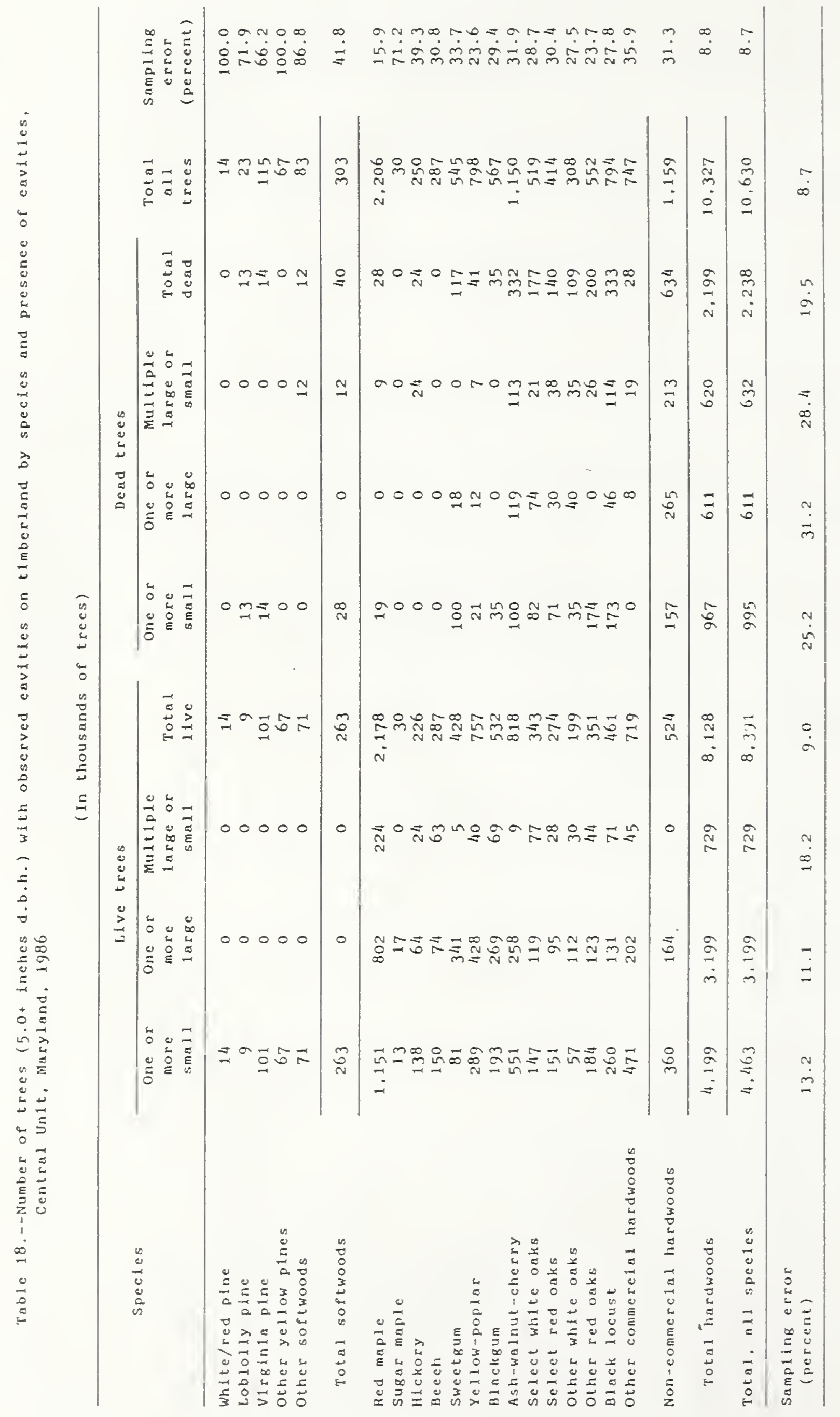


Table 19.--Number of seedlings, saplings, and shrubs on timberland by species and stand-size class, Central Unit, Maryland, 1986

\begin{tabular}{|c|c|c|c|c|c|c|}
\hline \multirow{2}{*}{ species } & \multicolumn{4}{|c|}{ Stand-size class } & \multirow{2}{*}{$\begin{array}{c}\text { AIl } \\
\text { classes }\end{array}$} & \multirow{2}{*}{$\begin{array}{l}\text { Sampling } \\
\text { error } \\
\text { (percent) }\end{array}$} \\
\hline & Sawtimber & Poletimber & seedling & Nonstocked & & \\
\hline Loblolly pine & 1 & 2 & 0 & 0 & 3 & 66.2 \\
\hline Other pine species & 6 & 3 & 15 & 0 & 24 & 31.7 \\
\hline other softwoods & 3 & 4 & 1 & 0 & 8 & 38.8 \\
\hline Total conlferous species & 11 & 9 & 16 & 0 & 35 & 24.4 \\
\hline Red maple & 343 & 72 & 61 & 0 & 477 & 14.1 \\
\hline Sugar maple & 0 & 3 & 0 & 0 & 3 & 73.1 \\
\hline Other maple species & 23 & 1 & 11 & 0 & 36 & 45.7 \\
\hline A 1 der species & 10 & 1 & 0 & 0 & 11 & 44.5 \\
\hline Serviceberry & 24 & 15 & 0 & 0 & 40 & $33 \cdot 7$ \\
\hline Azalea species & 30 & 4 & 0 & 0 & 34 & 20.7 \\
\hline Common pawpaw & 81 & 0 & 0 & 0 & 81 & 49.4 \\
\hline Birch species & 60 & 2 & 1 & 0 & 62 & 54.3 \\
\hline Hickory species & 74 & 23 & 1 & 0 & 98 & 18.1 \\
\hline Dogwood species & 222 & 22 & 2 & 0 & 246 & 15.2 \\
\hline American beech & 87 & 13 & 0 & 0 & 101 & 25.4 \\
\hline Ash species & 69 & 9 & 21 & 0 & 100 & 22.8 \\
\hline Huckleberry & 11 & 0 & 0 & 0 & 11 & 40.8 \\
\hline Witch-haze 1 & 6 & 0 & 0 & 0 & 6 & $51 \cdot 5$ \\
\hline American hol y & 36 & 27 & 1 & 0 & 64 & 32.7 \\
\hline Laurel species & 35 & 11 & 3 & 0 & 49 & 22.0 \\
\hline Common spicebush & 98 & 11 & 2 & 0 & 111 & 15.6 \\
\hline Swe tgum & 170 & 50 & 38 & 0 & 258 & 17.2 \\
\hline Yellow-poplar & 129 & 25 & 13 & 0 & 167 & 33.4 \\
\hline Magnolia species & 30 & 2 & 0 & 0 & 32 & $59 \cdot 3$ \\
\hline Tupelo species & 238 & 74 & 2 & 0 & 314 & 16.8 \\
\hline Black cherry & 276 & 91 & 23 & 0 & 390 & 22.1 \\
\hline Other cherry specles & 17 & 4 & 13 & 0 & 35 & 33.8 \\
\hline White oak & 67 & 10 & 8 & 0 & 86 & 24.7 \\
\hline Chestnut oak & 103 & 8 & 0 & 0 & 111 & 30.3 \\
\hline other white oaks & 16 & 1 & 0 & 0 & 17 & 75.4 \\
\hline Northern red oak & 42 & 5 & 0 & 0 & 48 & 28.1 \\
\hline Other black oaks & 90 & 17 & 5 & 0 & 113 & 20.4 \\
\hline Rhododendron species & 1 & 0 & 0 & 0 & 1 & 100.0 \\
\hline Rose species & 62 & 15 & 17 & 0 & 94 & 23.2 \\
\hline Rubus species & 134 & 35 & 49 & 0 & 218 & 10.4 \\
\hline Sassafras & 125 & 22 & 13 & 0 & 160 & 16.9 \\
\hline Blueberry & 121 & 35 & 0 & 0 & 157 & 12.8 \\
\hline Elm species & 52 & 5 & 2 & 0 & 59 & 31.8 \\
\hline Maple-leaved viburnum & 26 & 4 & 0 & 0 & 30 & 27.4 \\
\hline ArrowwoOd & 54 & 11 & 3 & 0 & 67 & $16 \cdot 3$ \\
\hline Other viburnum species & 36 & 9 & 0 & 0 & 45 & $19 \cdot 5$ \\
\hline Other deciduous species & 115 & 50 & 23 & 0 & 188 & $18 \cdot 3$ \\
\hline Total deciduous species & 3.115 & 690 & 315 & 0 & 4,120 & 6.6 \\
\hline Unknown species & 141 & 27 & 5 & 0 & 174 & 11.6 \\
\hline Total, all species & 3.267 & 727 & 336 & 0 & 4.330 & 6.4 \\
\hline $\begin{array}{l}\text { Sampling error } \\
\text { (percent) }\end{array}$ & $7 \cdot 9$ & 22.6 & 24.4 & .0 & 6.4 & \\
\hline
\end{tabular}




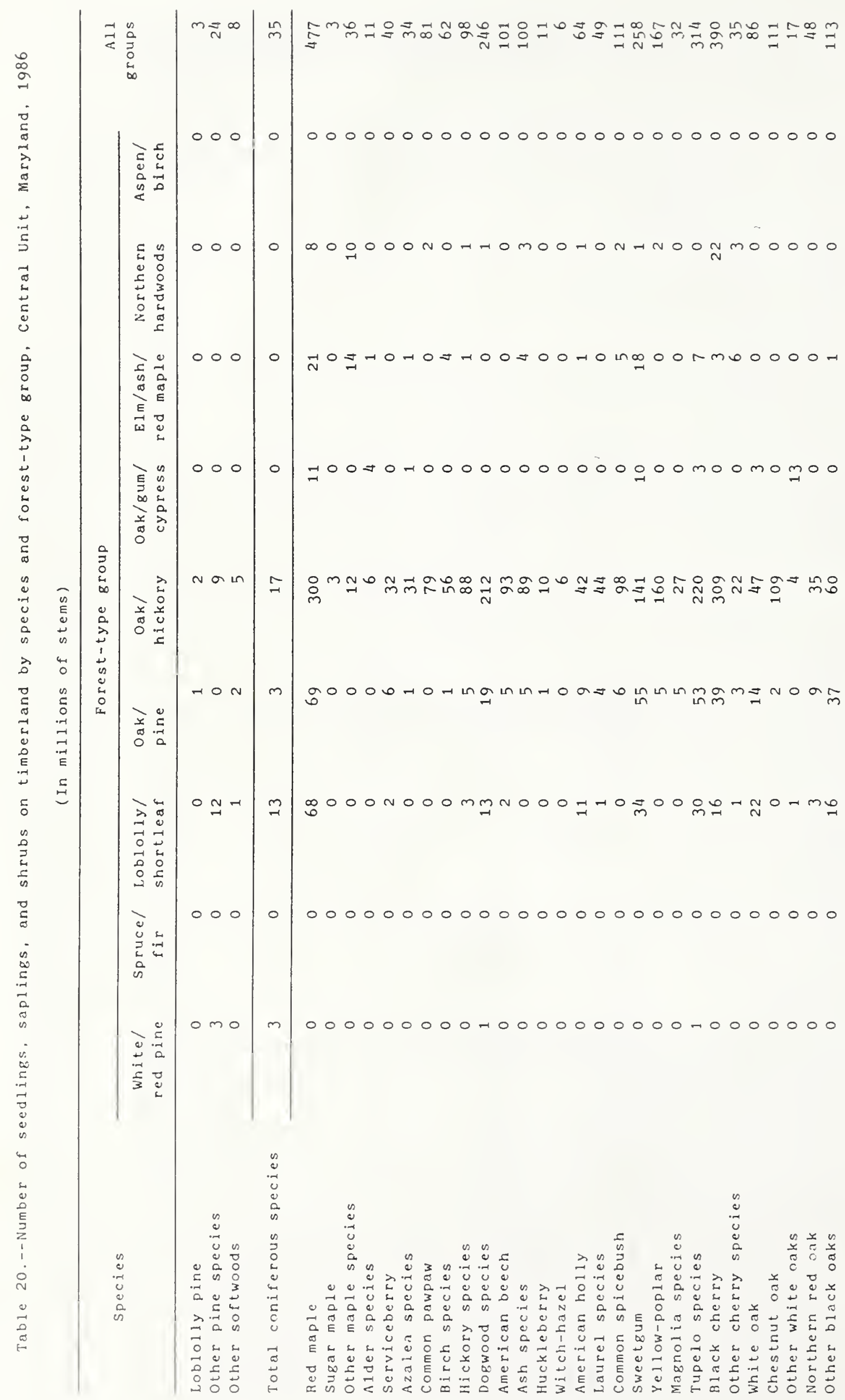




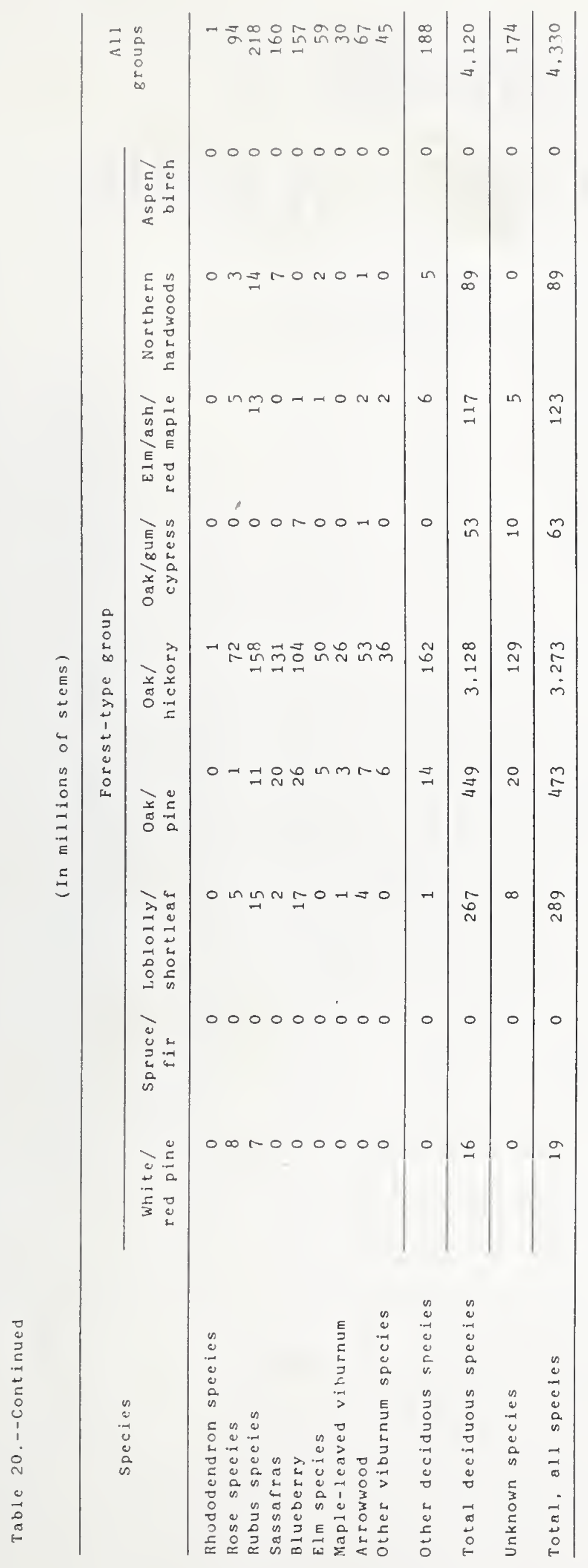


Table 21.--Number of seedlings, saplings, and shrubs on timberland by species and browse-utilization class, Central Unit, Maryland, 1986

(In millions of stems)

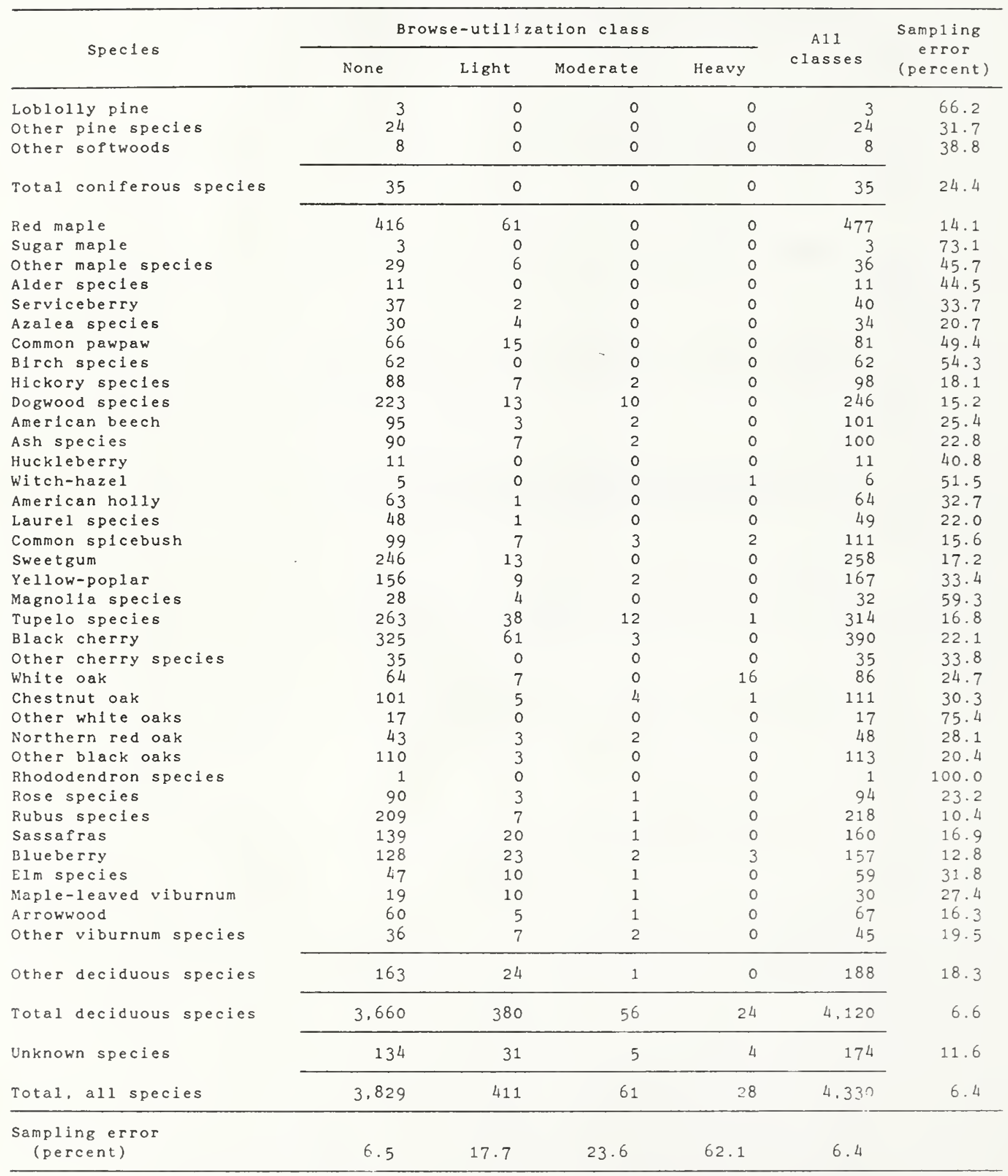


MARYLAND

\section{LOWER EASTERN SHORE} UNIT TABLES

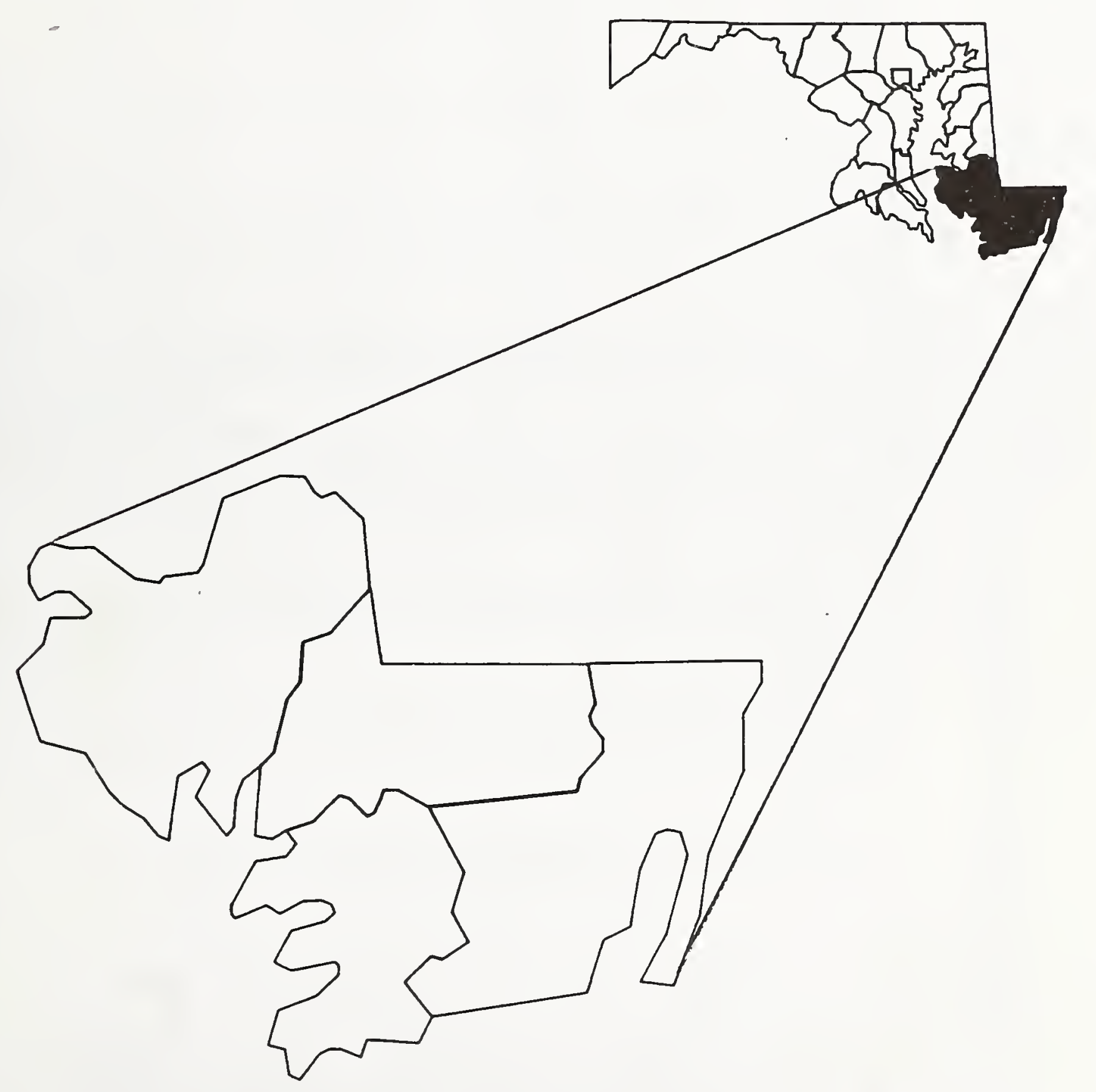











\begin{tabular}{|c|c|c|c|c|c|}
\hline \multirow{3}{*}{$\begin{array}{l}\text { Stand-size class } \\
\text { and type of stem }\end{array}$} & \multicolumn{4}{|c|}{ Mast type } & \multirow{3}{*}{$\begin{array}{l}\text { Tota } 1 \\
\text { stems }\end{array}$} \\
\hline & \multicolumn{3}{|c|}{ other } & \multirow{2}{*}{$\begin{array}{c}\text { Other } \\
\text { species }\end{array}$} & \\
\hline & Nuts & seeds & Berries & & \\
\hline \multicolumn{6}{|l|}{ SawtImber: } \\
\hline Shrubs & 0 & 19.239 & 138.386 & 212.625 & 370.250 \\
\hline Saplings & 12,601 & 59.514 & 37.175 & 0 & 109.290 \\
\hline Total sawtimber & 12,601 & 78.753 & 175.561 & 212.625 & 479.540 \\
\hline \multicolumn{6}{|l|}{ Poletimber: } \\
\hline Shrubs & 0 & 9.957 & 60.861 & 62.690 & 133.507 \\
\hline Saplings & 3.585 & 62.652 & 17.705 & 0 & 83.942 \\
\hline Total poletimber & 3.585 & 72,609 & 78.566 & 62.690 & 217.450 \\
\hline \multicolumn{6}{|l|}{ Sapling/seed 1 ing: } \\
\hline Shrubs & 0 & 3.584 & 50.772 & 46.531 & 100.886 \\
\hline Saplings & 1.634 & 32.135 & 3.890 & 0 & 37.658 \\
\hline Total sapling/seedling & 1.634 & 35.718 & 54.661 & 46.531 & 138.545 \\
\hline Total, all classes & 17.820 & 187.080 & 308.789 & 321.845 & 835.534 \\
\hline
\end{tabular}




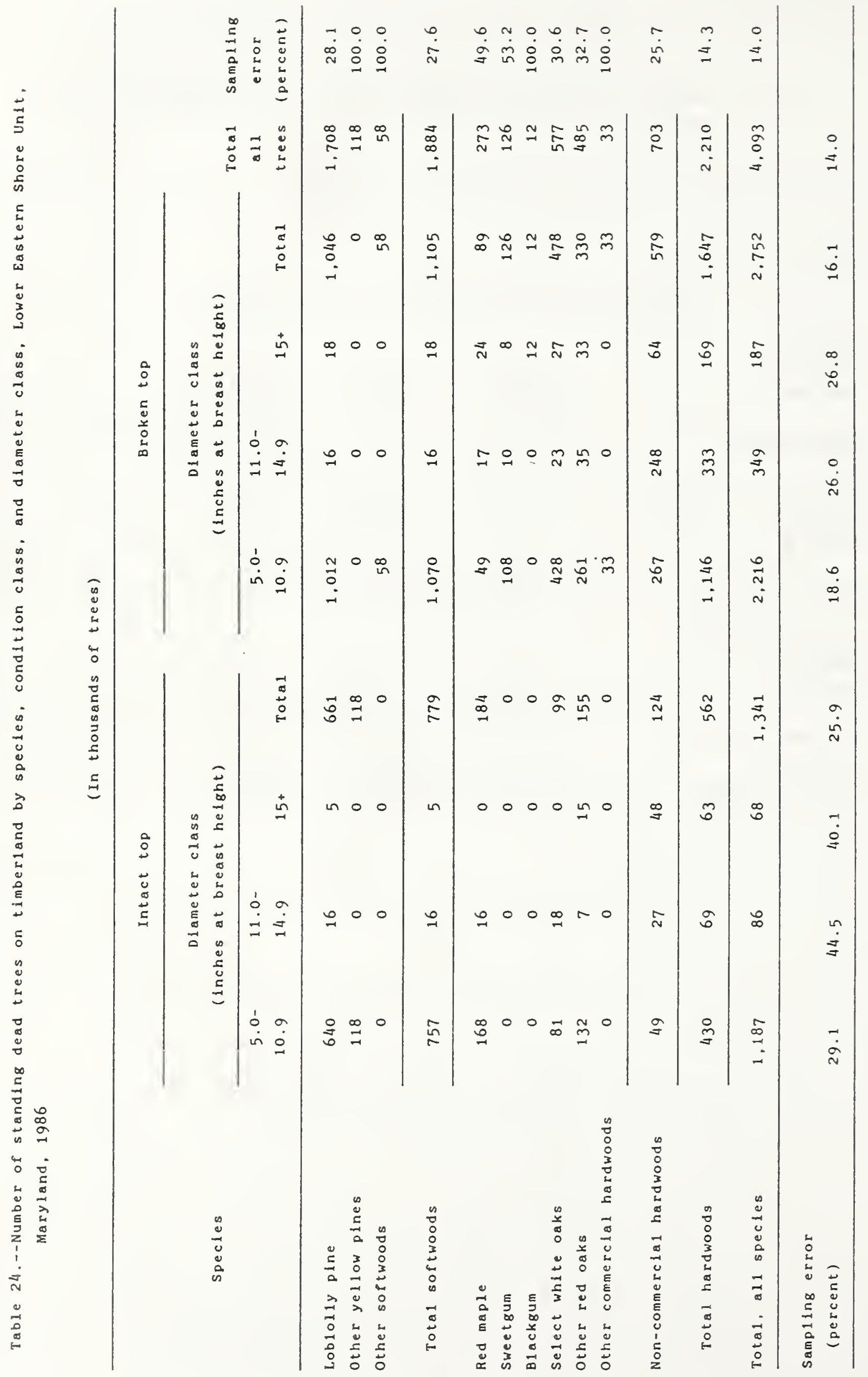




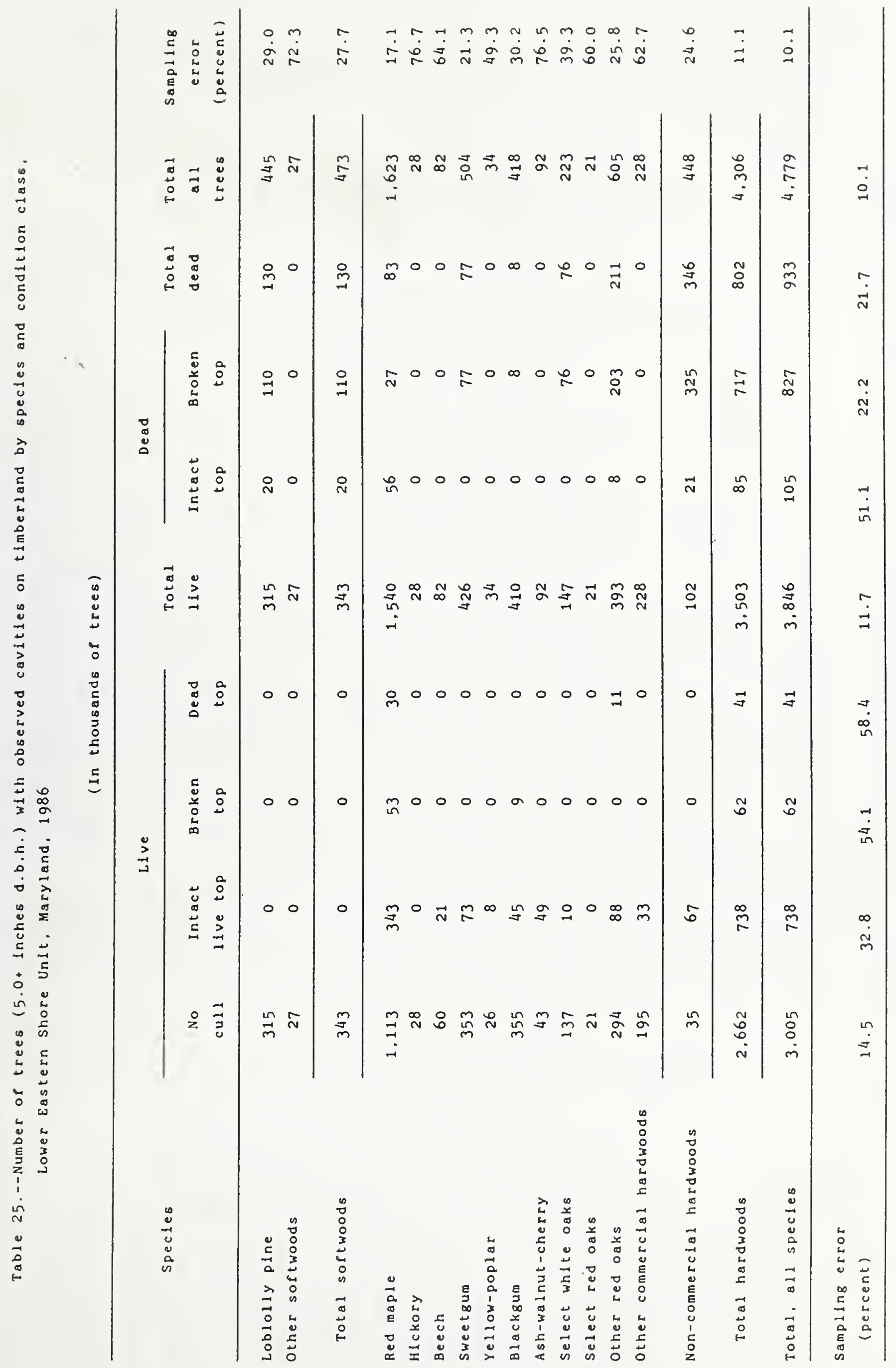






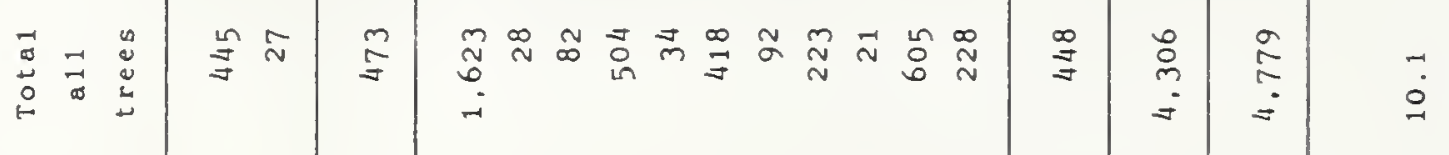

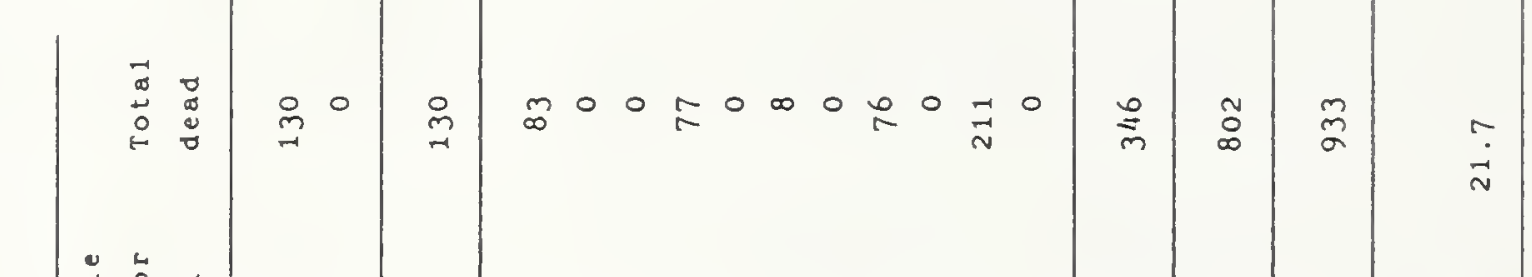







$\pm \infty \quad \Xi$

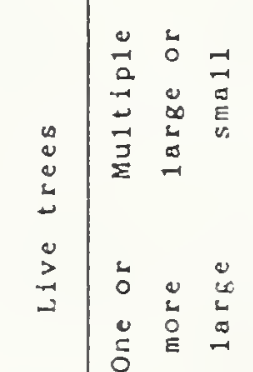


Table 27.--Number of seed11ngs. saplings, and shrubs on timberland by species and stand-size class. Lower Eastern Shore Unit. Maryland. 1986

(In m1111ons of stems)

\begin{tabular}{|c|c|c|c|c|c|c|}
\hline \multirow{2}{*}{ species } & \multicolumn{4}{|c|}{ stand-size class } & \multirow{2}{*}{$\begin{array}{c}\text { All } \\
\text { c1asses }\end{array}$} & \multirow{2}{*}{$\begin{array}{c}\text { Sampling } \\
\text { error } \\
\text { (percent) }\end{array}$} \\
\hline & Sawt imber & Polet $1 \mathrm{mber}$ & seed11ng & Nonstocked & & \\
\hline Lob1olly pine & 53 & 40 & 62 & 0 & 155 & $21 \cdot 8$ \\
\hline Other pine specles & 0 & 0 & 3 & 0 & 3 & $87 \cdot 3$ \\
\hline Other softwoods & 2 & 0 & 1 & 0 & 3 & $45 \cdot 7$ \\
\hline Total conlferous species & 56 & 40 & 65 & 0 & 161 & 21.1 \\
\hline Red maple & 131 & 125 & 201 & 0 & 459 & $15 \cdot 3$ \\
\hline Alder'species & 3 & 3 & 0 & 0 & 6 & $71 \cdot 5$ \\
\hline Serviceberry & 7 & 3 & 3 & 0 & 13 & 43.7 \\
\hline Azalea species & 13 & 5 & 2 & 0 & 20 & 25.4 \\
\hline HIckory species & 2 & 0 & 0 & 0 & 2 & 81.6 \\
\hline Dogwood spectes & 7 & 10 & 0 & 0 & 17 & 65.2 \\
\hline American beech & 3 & 0 & 0 & 0 & 3 & 59.6 \\
\hline Ash specles & 2 & 0 & 0 & 0 & 2 & 73.8 \\
\hline American holly & 93 & 41 & 25 & 0 & 159 & 15.2 \\
\hline Laurel specles & 2 & 1 & 0 & 0 & 3 & 52.2 \\
\hline Common spicebush & 2 & 0 & 0 & 0 & 2 & 71.8 \\
\hline Swe e $t g u m$ & 132 & 48 & 55 & 0 & 235 & 13.4 \\
\hline Yellow-poplar & 3 & 0 & 6 & 0 & 9 & 74.0 \\
\hline Magnolia species & 49 & 5 & 3 & 0 & 57 & $35 \cdot 3$ \\
\hline Tupelo species & 71 & 31 & 36 & 0 & 139 & 22.4 \\
\hline Black cherry & 10 & 2 & 2 & 0 & 14 & $35 \cdot 9$ \\
\hline Other cherry species & 2 & 0 & 0 & 0 & 2 & 100.0 \\
\hline White oak & 7 & 13 & 3 & 0 & 22 & 39.6 \\
\hline Other white oaks & 5 & 4 & 2 & 0 & 10 & 44.1 \\
\hline Northern red oak & 0 & 0 & 0 & 0 & 0 & 100.0 \\
\hline Other black oaks & 57 & 29 & 16 & 0 & 102 & $21 \cdot 3$ \\
\hline Rose specles & 2 & 0 & 1 & 0 & 4 & 47.6 \\
\hline Rubus species & 7 & 2 & 19 & 0 & 28 & $25 \cdot 5$ \\
\hline Sassafras & 19 & 3 & 19 & 0 & 41 & 22.7 \\
\hline Blueberry & 119 & 52 & 25 & 0 & 196 & $7 \cdot 1$ \\
\hline Elm species & 0 & 6 & 0 & 0 & 6 & 100.0 \\
\hline Maple-leaved viburnum & 1 & 0 & 0 & 0 & 1 & 100.0 \\
\hline Arrowwood & 3 & 4 & 1 & 0 & 8 & $33 \cdot 7$ \\
\hline Other viburnum species & 1 & 1 & 0 & 0 & 1 & $70 \cdot 9$ \\
\hline other deciduous species & 27 & 8 & 22 & 0 & 56 & 15.7 \\
\hline Total deciduous species & 781 & 398 & 442 & 0 & 1.621 & $7 \cdot 2$ \\
\hline Unknown species & 221 & 57 & 35 & 0 & 314 & $8 \cdot 3$ \\
\hline Total, all species & 1.058 & 496 & 542 & 0 & 2.096 & $6 \cdot 5$ \\
\hline $\begin{array}{l}\text { Sampling error } \\
\text { (percent) }\end{array}$ & 9.6 & 18.6 & 24.1 & .0 & 6.5 & \\
\hline
\end{tabular}




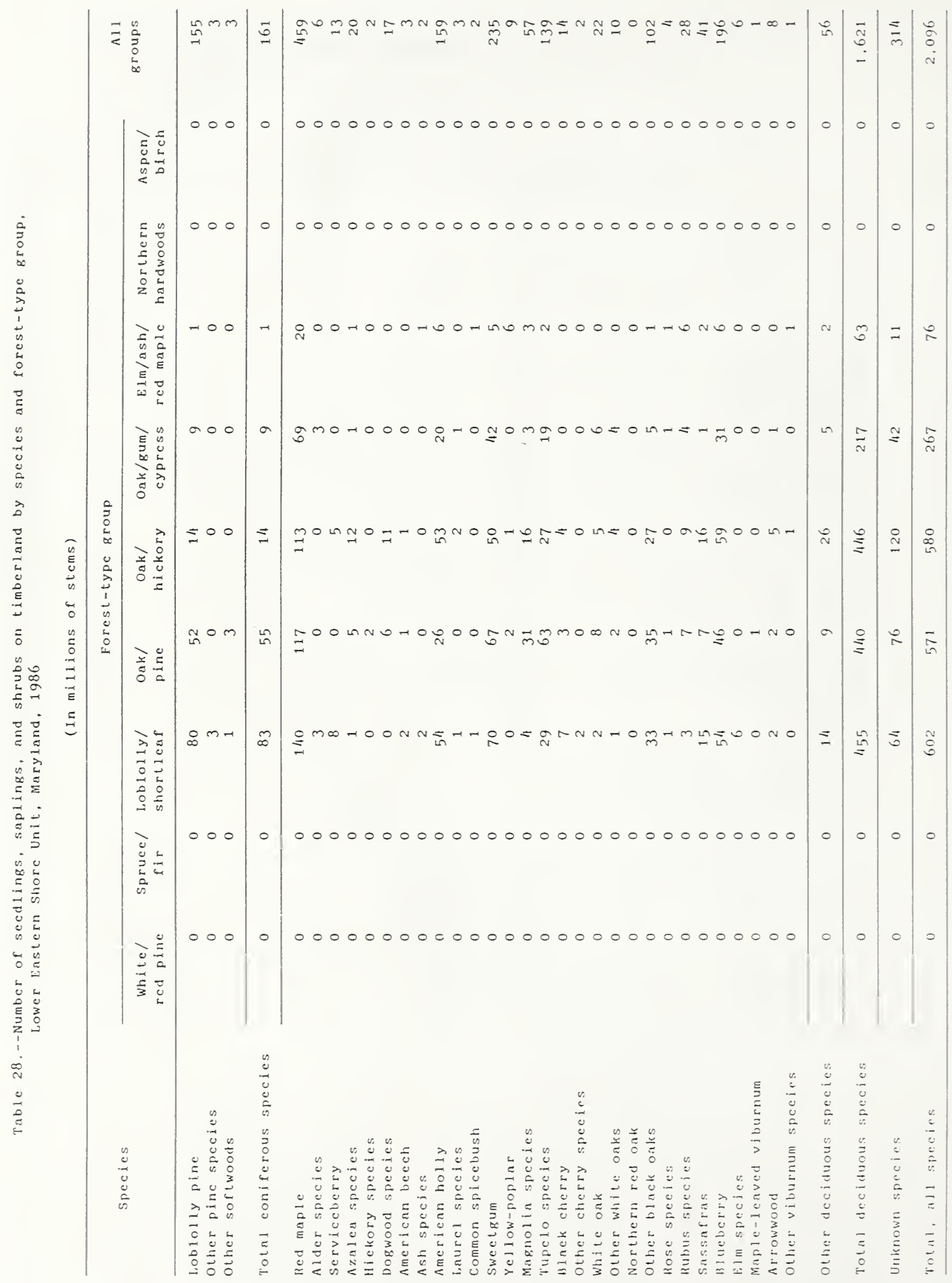


Table 29.-Number of seedlings, saplings, and shrubs on tlmberland by species and browse-utilization class, Lower Eastern Shore Unit, Maryland, 1986

\begin{tabular}{|c|c|c|c|c|c|c|}
\hline \multirow{2}{*}{ Species } & \multicolumn{4}{|c|}{ Browse-utilization c1ass } & \multirow{2}{*}{$\begin{array}{c}\text { A11 } \\
\text { classes }\end{array}$} & \multirow{2}{*}{$\begin{array}{l}\text { Sampling } \\
\text { error } \\
\text { (percent) }\end{array}$} \\
\hline & None & Light & Moderate & Heavy & & \\
\hline Loblolly pine & 155 & 0 & 0 & 1 & 155 & 21.8 \\
\hline Other pine species & 3 & 0 & 0 & 0 & 3 & $87 \cdot 3$ \\
\hline Other softwoods & 3 & 0 & 0 & 0 & 3 & $45 \cdot 7$ \\
\hline Total coniferous specles & 161 & 0 & 0 & 1 & 161 & 21.1 \\
\hline Red maple & 411 & 44 & 2 & 1 & 459 & $15 \cdot 3$ \\
\hline Alder specles & 4 & 2 & 0 & 0 & 6 & $71 \cdot 5$ \\
\hline Serviceberry & 13 & 0 & 0 & 0 & 13 & 43.7 \\
\hline Azalea species & 17 & 1 & 2 & 0 & 20 & 25.4 \\
\hline Hickory specles & 2 & 0 & 0 & 0 & 2 & 81.6 \\
\hline Dogwood specles & 12 & 0 & 5 & 0 & 17 & 65.2 \\
\hline American beech & 3 & 0 & 0 & 0 & 3 & 59.6 \\
\hline Ash species & 2 & 0 & 0 & 0 & 2 & 73.8 \\
\hline Amerlcan holly & 146 & 2 & 10 & 1 & 159 & 15.2 \\
\hline Laure 1 species & 3 & 0 & 0 & 0 & 3 & 52.2 \\
\hline Common splcebush & 2 & 0 & 0 & 0 & 2 & 71.8 \\
\hline Sweet gum & 211 & 20 & 0 & 4 & 235 & 13.4 \\
\hline Yellow-poplar & 9 & 0 & 0 & 0 & 9 & 74.0 \\
\hline Magnolia species & 50 & 7 & 0 & 0 & 57 & $35 \cdot 3$ \\
\hline Tupelo species & 124 & 10 & 0 & 4 & 139 & 22.4 \\
\hline Black cherry & 12 & 1 & 1 & 0 & 14 & $35 \cdot 9$ \\
\hline Other cherry species & 0 & 0 & 0 & 2 & 2 & 100.0 \\
\hline White oak & 17 & 2 & 3 & 0 & 22 & 39.6 \\
\hline Other white oaks & 10 & 0 & 0 & 0 & 10 & 44.1 \\
\hline Northern red oak & 0 & 0 & 0 & 0 & 0 & 100.0 \\
\hline other black oaks & 88 & 10 & 3 & 0 & 102 & $21 \cdot 3$ \\
\hline Rose species & 3 & 1 & 0 & 0 & 4 & 47.6 \\
\hline Rubus species & 25 & 3 & 0 & 0 & 28 & $25 \cdot 5$ \\
\hline Sassafras & 33 & 4 & 4 & 0 & 41 & $22 \cdot 7$ \\
\hline Bluebersy & 131 & 52 & 11 & 2 & 196 & 7.1 \\
\hline Elm species & 4 & 2 & 0 & 0 & 6 & 100.0 \\
\hline Maple-leaved viburnum & 0 & 1 & 0 & 0 & 1 & 100.0 \\
\hline Arrowwood & 7 & 1 & 0 & 0 & 8 & $33 \cdot 7$ \\
\hline Other viburnum species & 1 & 0 & 0 & 0 & 1 & 70.9 \\
\hline Other deciduous species & 52 & 3 & 1 & 1 & 56 & $15 \cdot 7$ \\
\hline Total deciduous species & 1.396 & 167 & 43 & 15 & 1.621 & 7.2 \\
\hline Unknown species & 242 & 58 & 12 & 2 & 314 & $8 \cdot 3$ \\
\hline Total, all species & 1.798 & 225 & 55 & 17 & 2,096 & 6.5 \\
\hline $\begin{array}{l}\text { Sampling error } \\
\text { (percent) }\end{array}$ & 6.7 & 14.0 & 23.0 & 42.9 & 6.5 & \\
\hline
\end{tabular}




\section{MARYLAND \\ SOUTHERN UNIT TABLES}

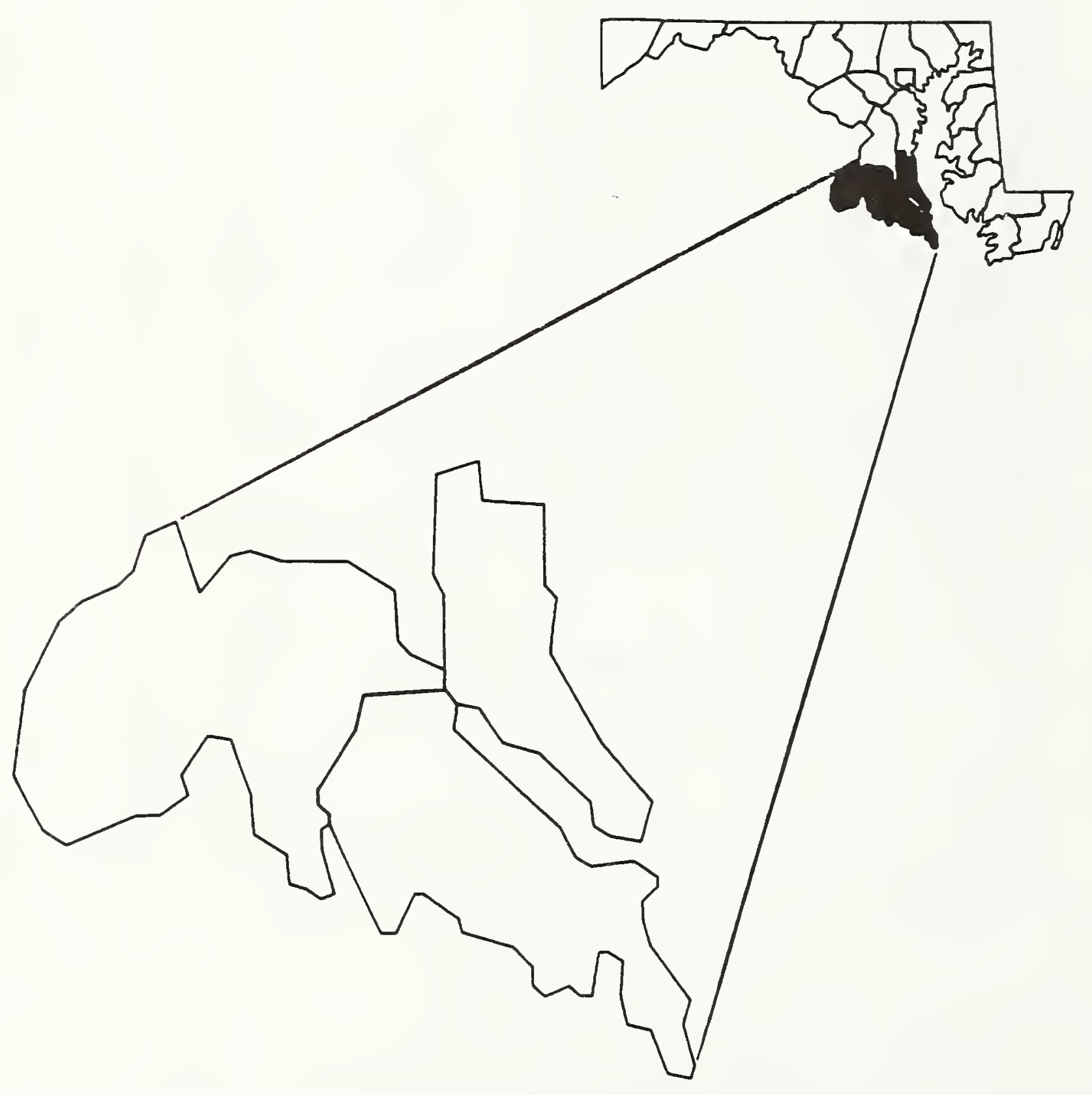




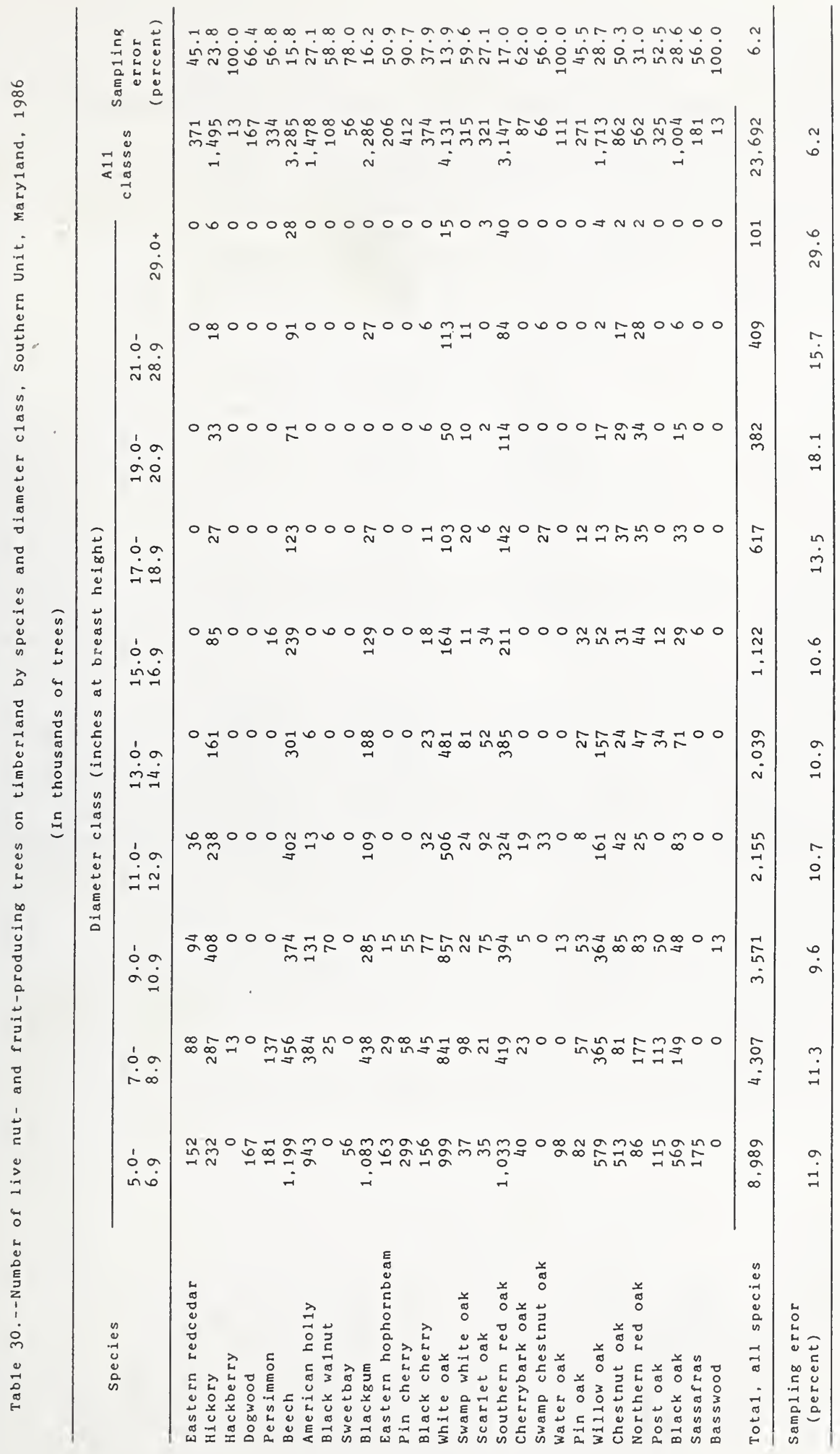


Table 31.--Number of shrubs and saplings on timberland by stand-size class, type of stem, and mast type, Southern Unit, Maryland, 1986

(In thousands of stems)

\begin{tabular}{|c|c|c|c|c|c|}
\hline \multirow{2}{*}{$\begin{array}{l}\text { stand-size class } \\
\text { and type of stem }\end{array}$} & \multicolumn{4}{|c|}{ Mast type } & \multirow{2}{*}{$\begin{array}{l}\text { Total } \\
\text { stems }\end{array}$} \\
\hline & Nuts & $\begin{array}{l}\text { other } \\
\text { seeds }\end{array}$ & Berries & $\begin{array}{c}\text { Other } \\
\text { species }\end{array}$ & \\
\hline \multicolumn{6}{|l|}{ Sawtimber: } \\
\hline Shrubs & 0 & 25.669 & 167.555 & 30,229 & 223.454 \\
\hline Saplings & 28.486 & 60.769 & 47.094 & 0 & $136 \cdot 348$ \\
\hline Total sawtimber & 28.486 & 86.438 & 214.650 & 30,229 & 359.802 \\
\hline \multicolumn{6}{|l|}{ Poletimber: } \\
\hline Shrubs & 0 & 1.025 & 24.481 & 3.143 & 28.650 \\
\hline Saplings & 6.221 & 9.527 & 2.848 & 0 & 18,596 \\
\hline Total poletimber & 6.221 & 10.552 & 27.329 & 3.143 & 47.246 \\
\hline \multicolumn{6}{|l|}{ Sapling/seedling: } \\
\hline Shrubs & 0 & 369 & 16.165 & 4.077 & 20.611 \\
\hline Saplings & 1.477 & 7.857 & 2.707 & 0 & 12.041 \\
\hline Total sapling/seedling & 1.477 & 8.226 & 18.871 & 4.077 & 32.652 \\
\hline Total, all classes & 36.184 & 105.216 & 260.851 & 37.449 & 439.700 \\
\hline
\end{tabular}




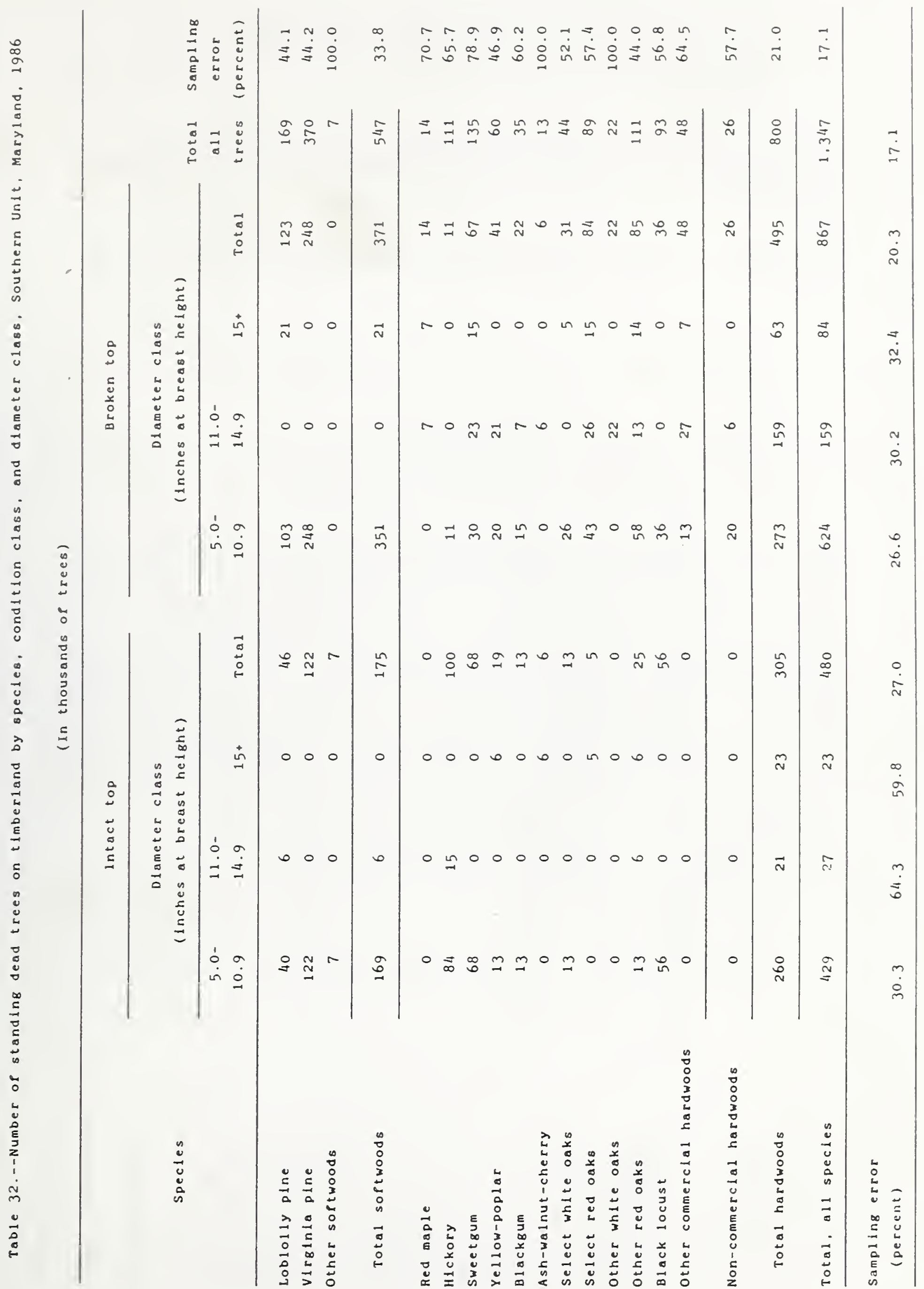




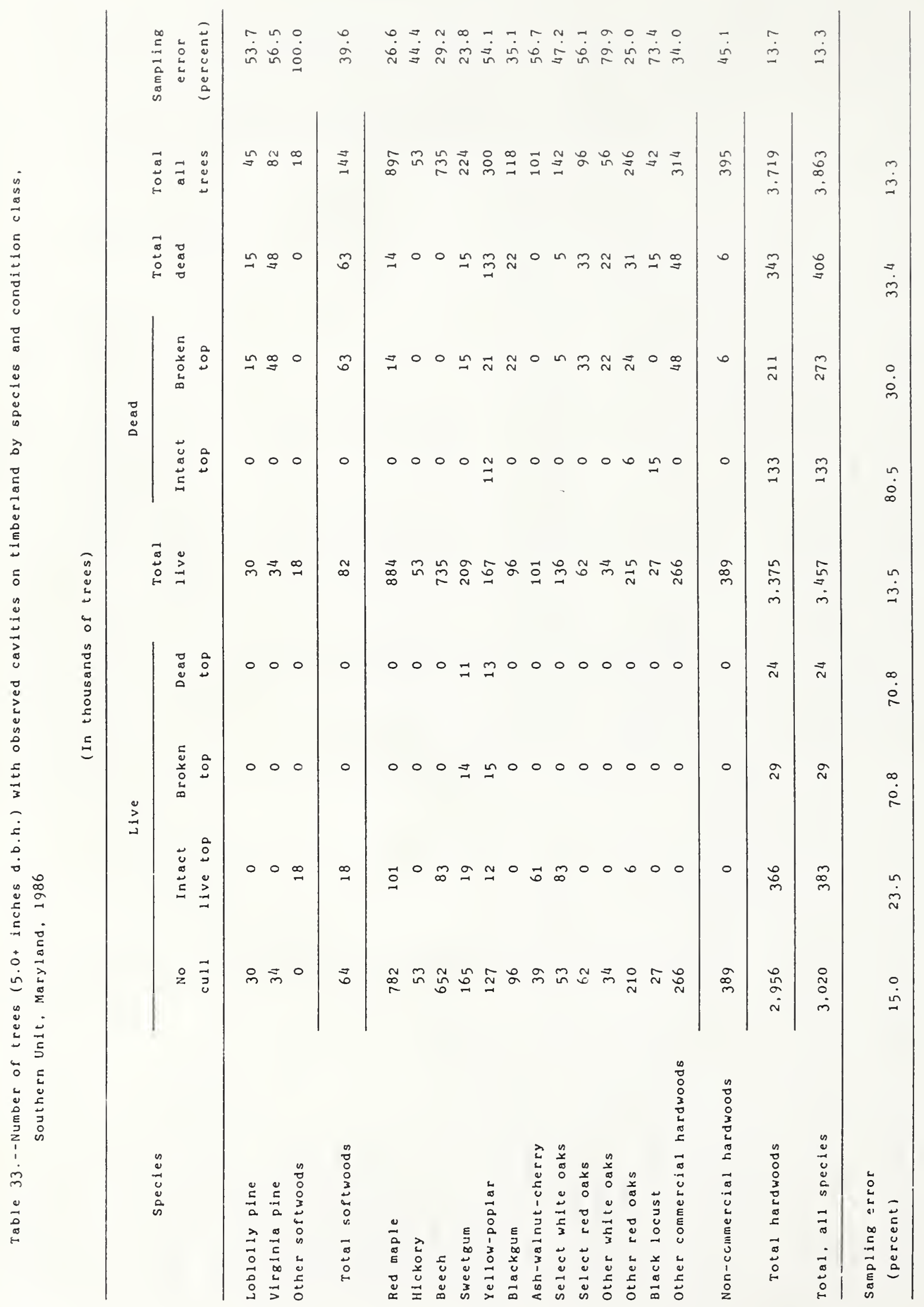







PERCENT OF SEEDLINGS, SAPLINGS, AND SHRUBS BY STAND-SIZE, SOUTHERN UNIT

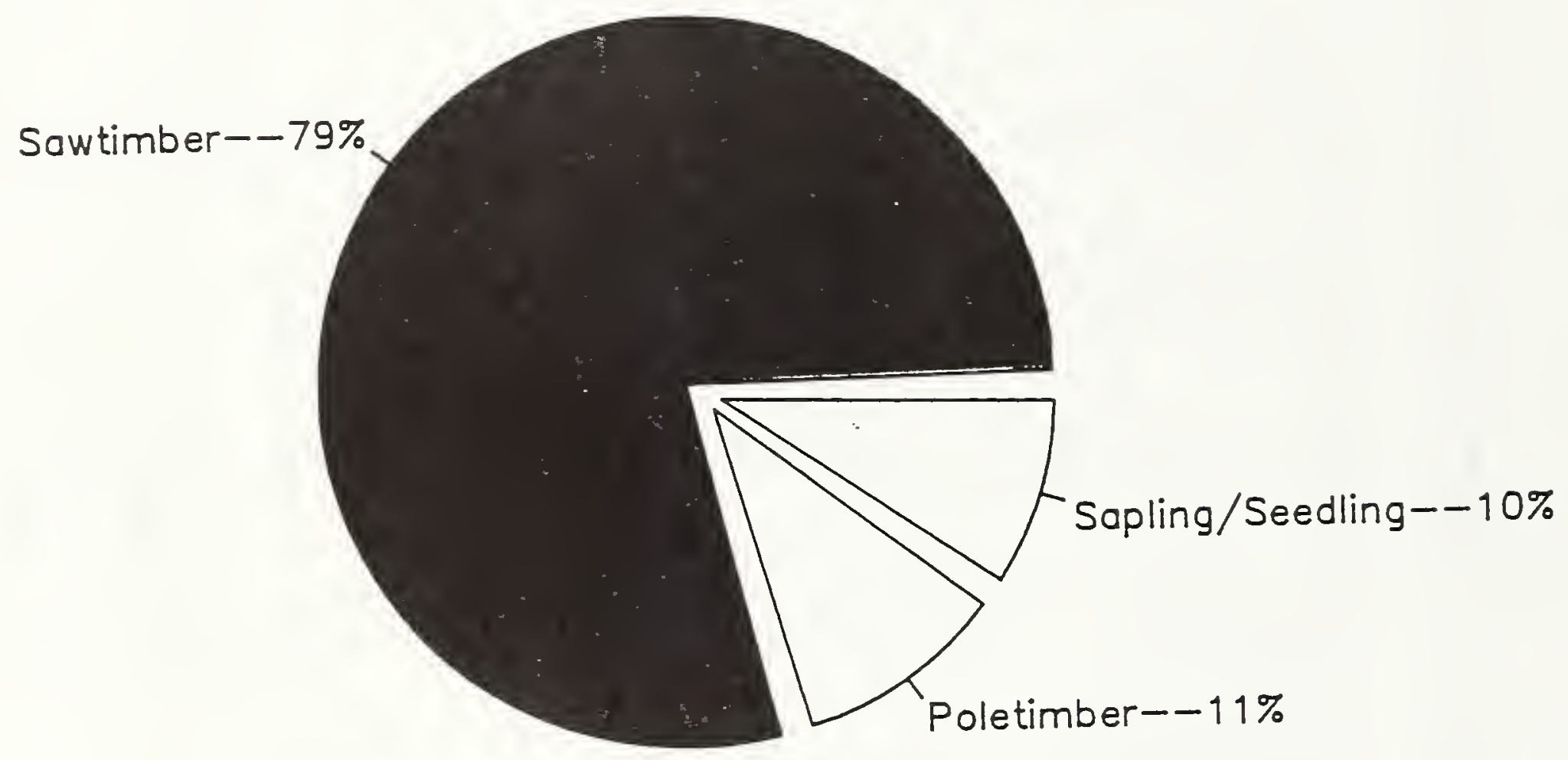


Table 35.--Number of seedlings. saplings, and shrubs on timberland by species and stand-size class. Southern Unit. Maryland, 1986

(In militions of stems)

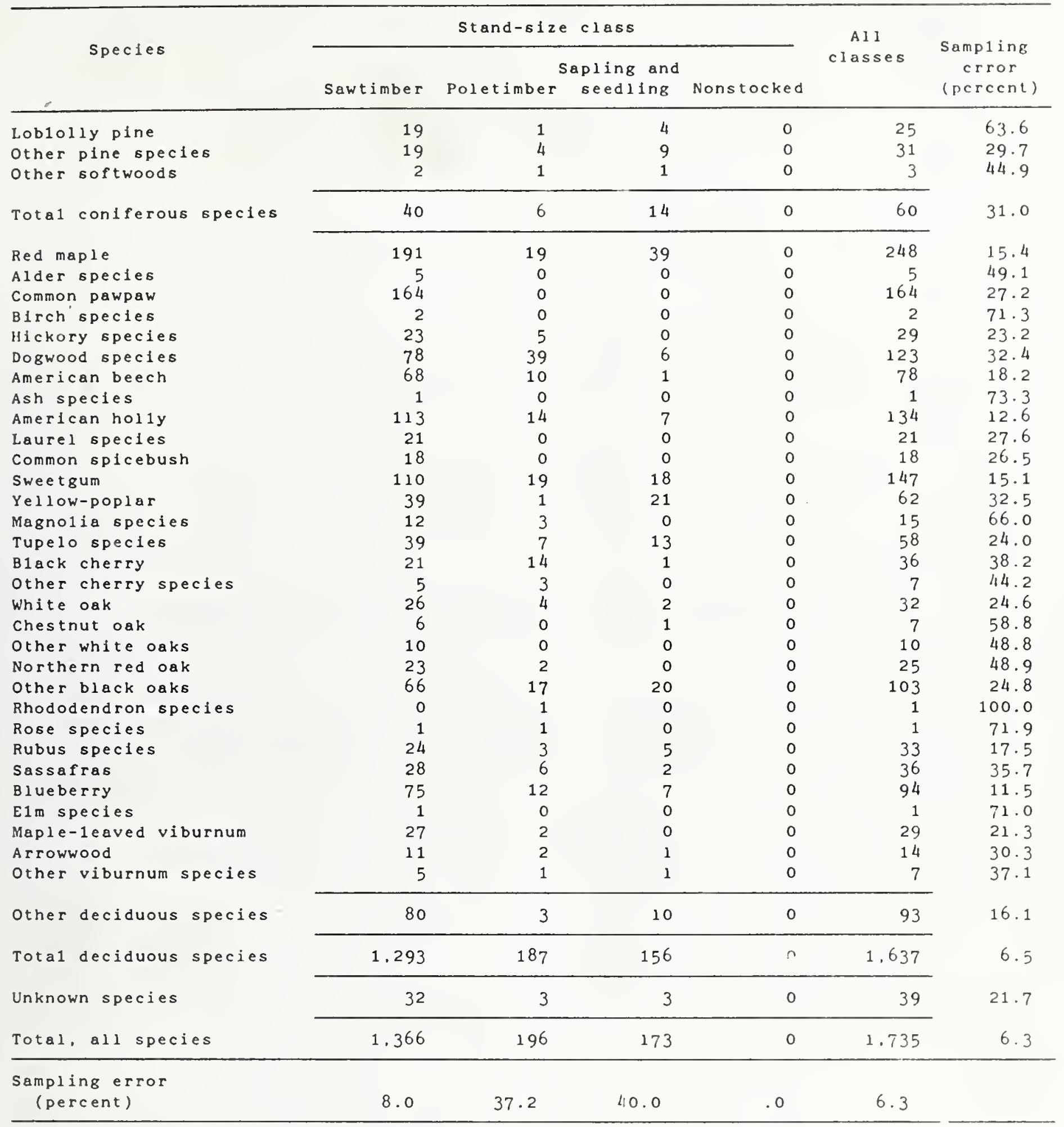












Table 37.--Number of seedlings, saplings, and shrubs on timberland by species and browse-utilization c1ass, Southern Unit, Maryland, 1986

\begin{tabular}{|c|c|c|c|c|c|c|}
\hline \multirow{2}{*}{ Species } & \multicolumn{4}{|c|}{ Browse-utilization c1ass } & \multirow{2}{*}{$\begin{array}{c}\text { All } \\
\text { classes }\end{array}$} & \multirow{2}{*}{$\begin{array}{c}\text { Sampling } \\
\text { error } \\
\text { (percent) }\end{array}$} \\
\hline & None & Light & Moderate & Heavy & & \\
\hline Lob1011y pine & 25 & 0 & 0 & 0 & 25 & 63.6 \\
\hline Other pine species & 31 & 0 & 0 & 0 & 31 & 29.7 \\
\hline other softwoods & 3 & 0 & 0 & 0 & 3 & 44.9 \\
\hline Total coniferous species & 60 & 0 & 0 & 0 & 60 & 31.0 \\
\hline Red map1e & 245 & 4 & 0 & 0 & 248 & 15.4 \\
\hline A1der species & 5 & 0 & 0 & 0 & 5 & 49.1 \\
\hline Common pawpaw & 155 & 9 & 0 & 0 & 164 & 27.2 \\
\hline Birch species & 2 & 0 & 0 & 0 & 2 & $71 \cdot 3$ \\
\hline Hickory species & 27 & 2 & 0 & 0 & 29 & 23.2 \\
\hline Dogwood species & 123 & 1 & 0 & 0 & 123 & 32.4 \\
\hline American beech & 69 & 9 & 0 & 1 & 78 & 18.2 \\
\hline Ash species & 1 & 0 & 0 & 0 & 1 & $73 \cdot 3$ \\
\hline American holly & 134 & 0 & 0 & 0 & 134 & 12.6 \\
\hline Laurel species & 21 & 0 & 0 & 0 & 21 & 27.6 \\
\hline Common spicebush & 18 & 0 & 0 & 0 & 18 & 26.5 \\
\hline Sweetgum & 144 & 3 & $\mathrm{Q}$ & 0 & 147 & $15 \cdot 1$ \\
\hline Ye11ow-poplar & 62 & 0 & 0 & 0 & 62 & $32 \cdot 5$ \\
\hline Magno1fa species & 15 & 0 & 0 & 0 & 15 & 66.0 \\
\hline Tupelo species & 57 & 1 & 0 & 0 & 58 & 24.0 \\
\hline Black cherry & 17 & 19 & 0 & 0 & 36 & 38.2 \\
\hline Other cherry species & 7 & 1 & 0 & 0 & 7 & 44.2 \\
\hline White oak & 32 & 0 & 0 & 0 & 32 & 24.6 \\
\hline Chestnut oak & 7 & 0 & 0 & 0 & 7 & 58.8 \\
\hline Other white oaks & 10 & 0 & 0 & 0 & 10 & 48.8 \\
\hline Northern red oak & 22 & 3 & 0 & 0 & 25 & 48.9 \\
\hline Other black oaks & 99 & 3 & 0 & 0 & 103 & 24.8 \\
\hline Rhododendron species & 1 & 0 & 0 & 0 & 1 & 100.0 \\
\hline Rose species & 1 & 0 & 0 & 0 & 1 & $71 \cdot 9$ \\
\hline Rubus species & 32 & 1 & 0 & 0 & 33 & $17 \cdot 5$ \\
\hline Sassafras & 23 & 13 & 0 & 0 & 36 & $35 \cdot 7$ \\
\hline Blueberry & 86 & 8 & 0 & 0 & 94 & $11 \cdot 5$ \\
\hline E1m species & 1 & 0 & 0 & 0 & 1 & 71.0 \\
\hline Maple-1eaved viburnum & 29 & 0 & 0 & 0 & 29 & $21 \cdot 3$ \\
\hline A r rowwood & 14 & 0 & 0 & 0 & 14 & $30 \cdot 3$ \\
\hline Other viburnum species & 7 & 0 & 1 & 0 & 7 & $37 \cdot 1$ \\
\hline Other deciduous species & 93 & 0 & 0 & 0 & 93 & 16.1 \\
\hline Tota1 deciduous species & 1.559 & 76 & 1 & 1 & 1.637 & 6.5 \\
\hline Unknown species & 38 & 1 & 0 & 0 & 39 & $21 \cdot 7$ \\
\hline Tota1, a11 species & 1.657 & 77 & 1 & 1 & 1.735 & $6 \cdot 3$ \\
\hline Samp1ing error & & & & & & \\
\hline (percent) & 6.7 & 25.0 & 100.0 & 100.0 & 6.3 & \\
\hline
\end{tabular}




$$
\begin{gathered}
\text { MARYLAND } \\
\text { WESTERN UNIT } \\
\text { TABLES }
\end{gathered}
$$

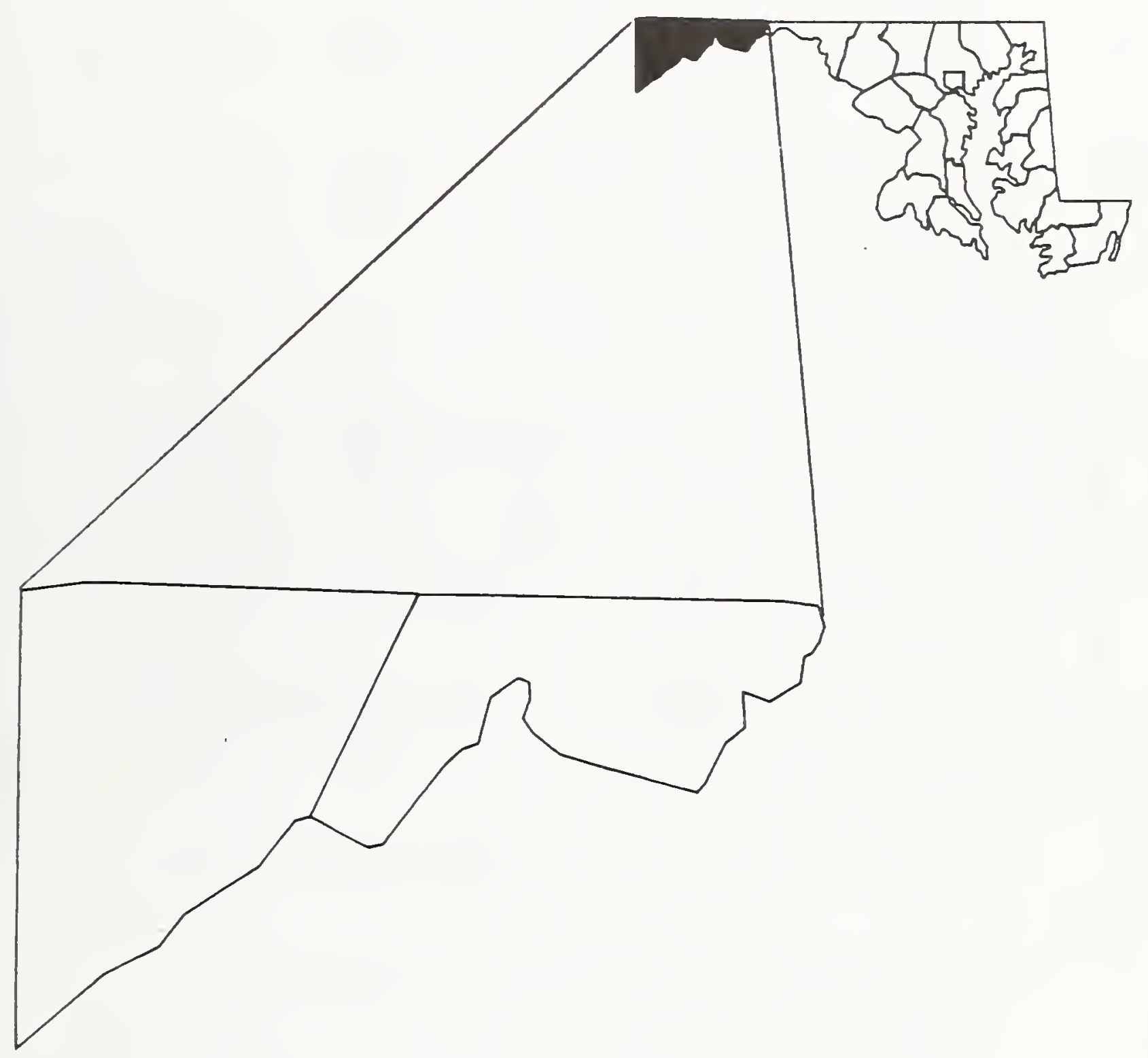




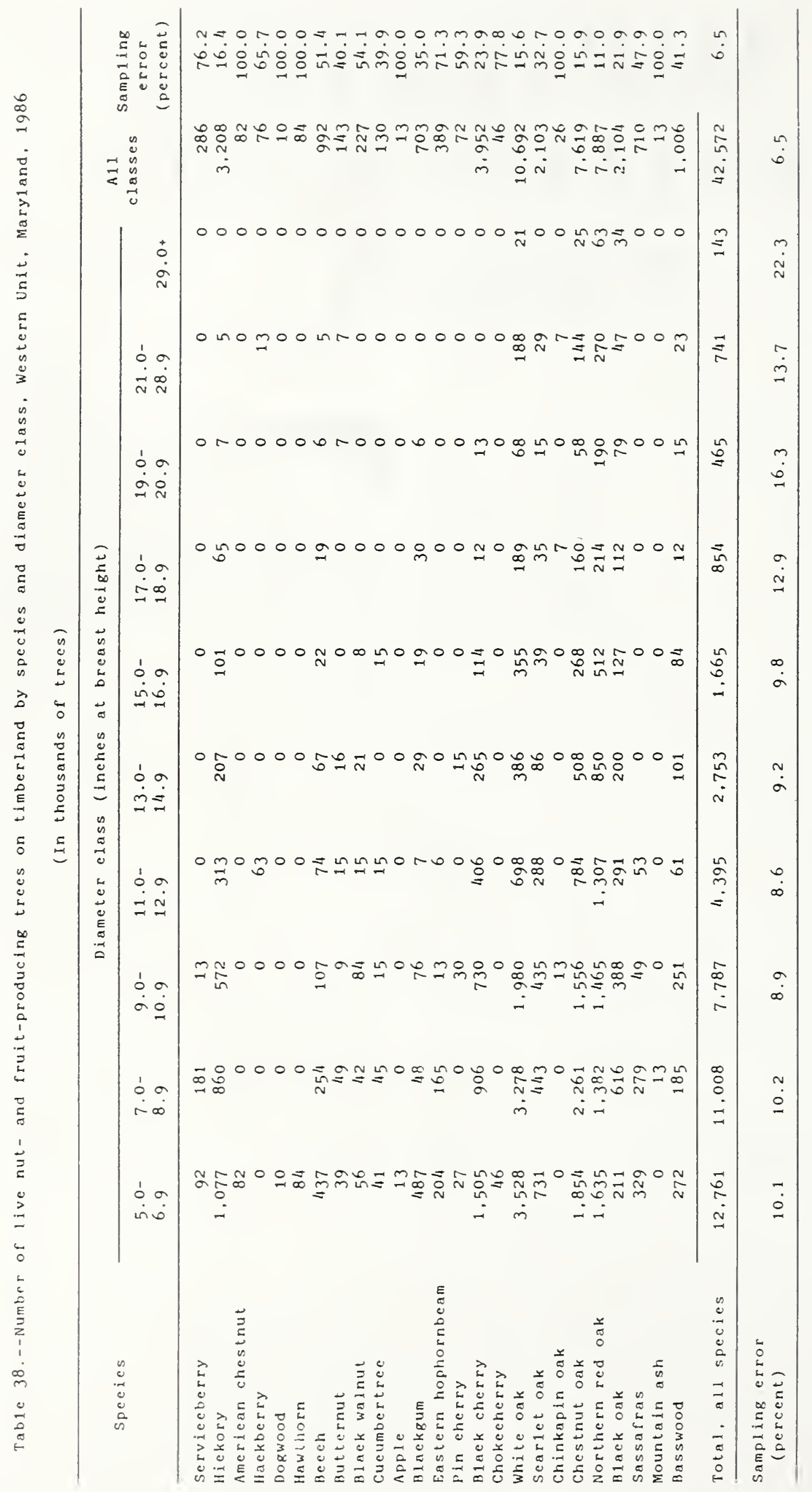


Table 39.--Number of shrubs and saplings on timberland by stand-size class, type of stem. and mast type, Western Unit, Maryland, 1986

( In thousands of stems)

\begin{tabular}{|c|c|c|c|c|c|}
\hline \multirow{2}{*}{$\begin{array}{l}\text { Stand-size class } \\
\text { and type of stem }\end{array}$} & \multicolumn{4}{|c|}{ Mast type } & \multirow{2}{*}{$\begin{array}{l}\text { Total } \\
\text { stems }\end{array}$} \\
\hline & Nuts & $\begin{array}{l}\text { other } \\
\text { seeds }\end{array}$ & Berrles & $\begin{array}{l}\text { Other } \\
\text { specles }\end{array}$ & \\
\hline \multicolumn{6}{|l|}{ Sawt 1 mber: } \\
\hline shrubs & 0 & 70.767 & 132.253 & 10.196 & 213.217 \\
\hline Saplings & 10.013 & 42.086 & 24.369 & 745 & 77.213 \\
\hline Total sawtimber & 10.013 & 112.854 & 156.621 & 10.941 & 290.430 \\
\hline \multicolumn{6}{|l|}{ Poletimber: } \\
\hline Shrubs & 2.198 & 23.536 & 76,203 & 3.530 & 105.467 \\
\hline Saplings & 13.070 & 24.581 & 17.585 & 0 & 55.237 \\
\hline Total poletimber & 15.268 & 48.117 & 93,789 & 3.530 & 160.704 \\
\hline \multicolumn{6}{|l|}{ Sapling/seedling: } \\
\hline Shrubs & 0 & 6.170 & 29.501 & 682 & 36.353 \\
\hline Saplings & 8.783 & 21.331 & 10.213 & 1.604 & 41.931 \\
\hline Total sapling/seedling & 8.783 & 27.501 & 39.714 & 2.286 & 78.285 \\
\hline Total, a11 classes & 34.065 & 188.472 & 290.124 & 16.757 & 529.419 \\
\hline
\end{tabular}




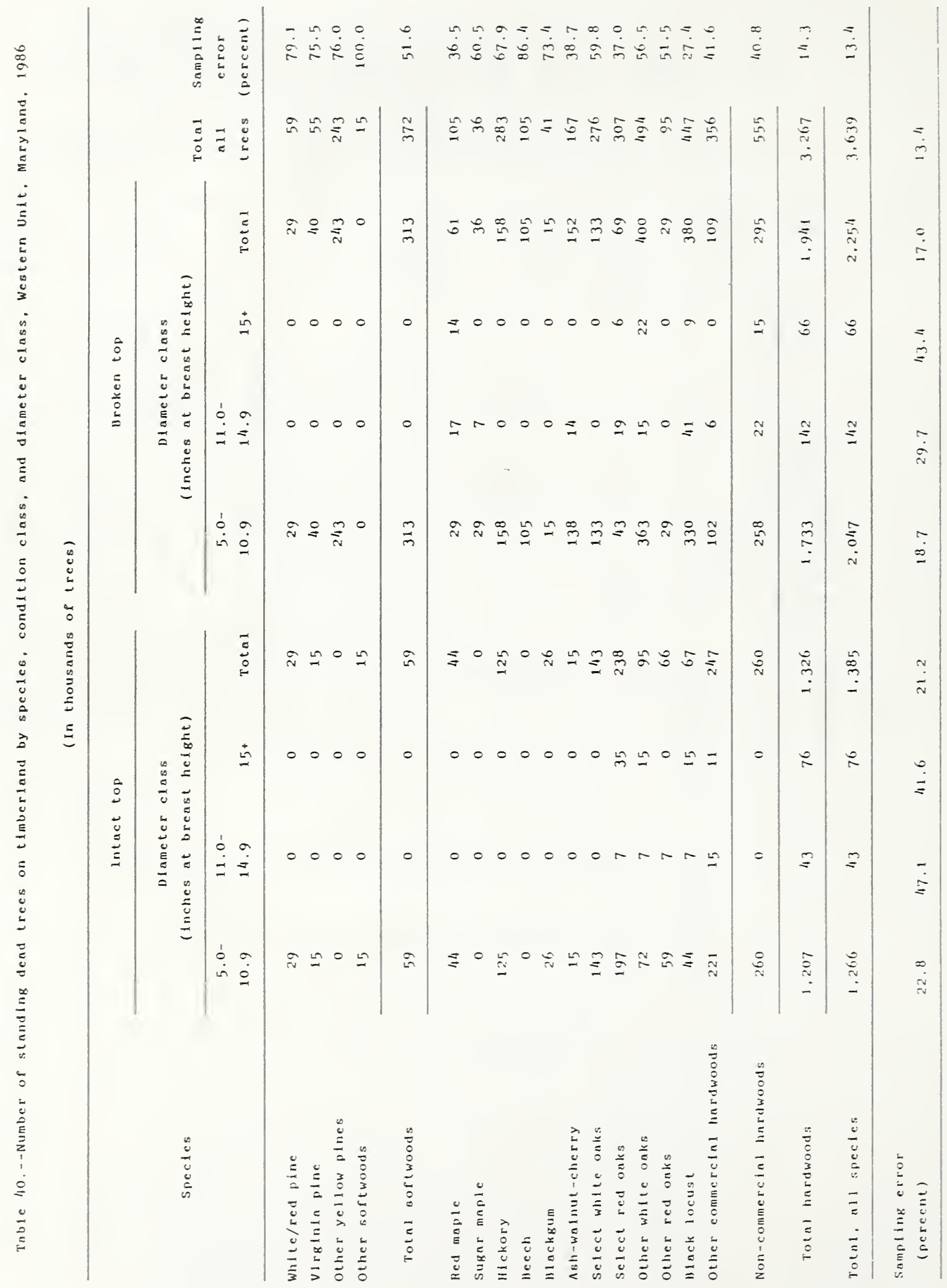




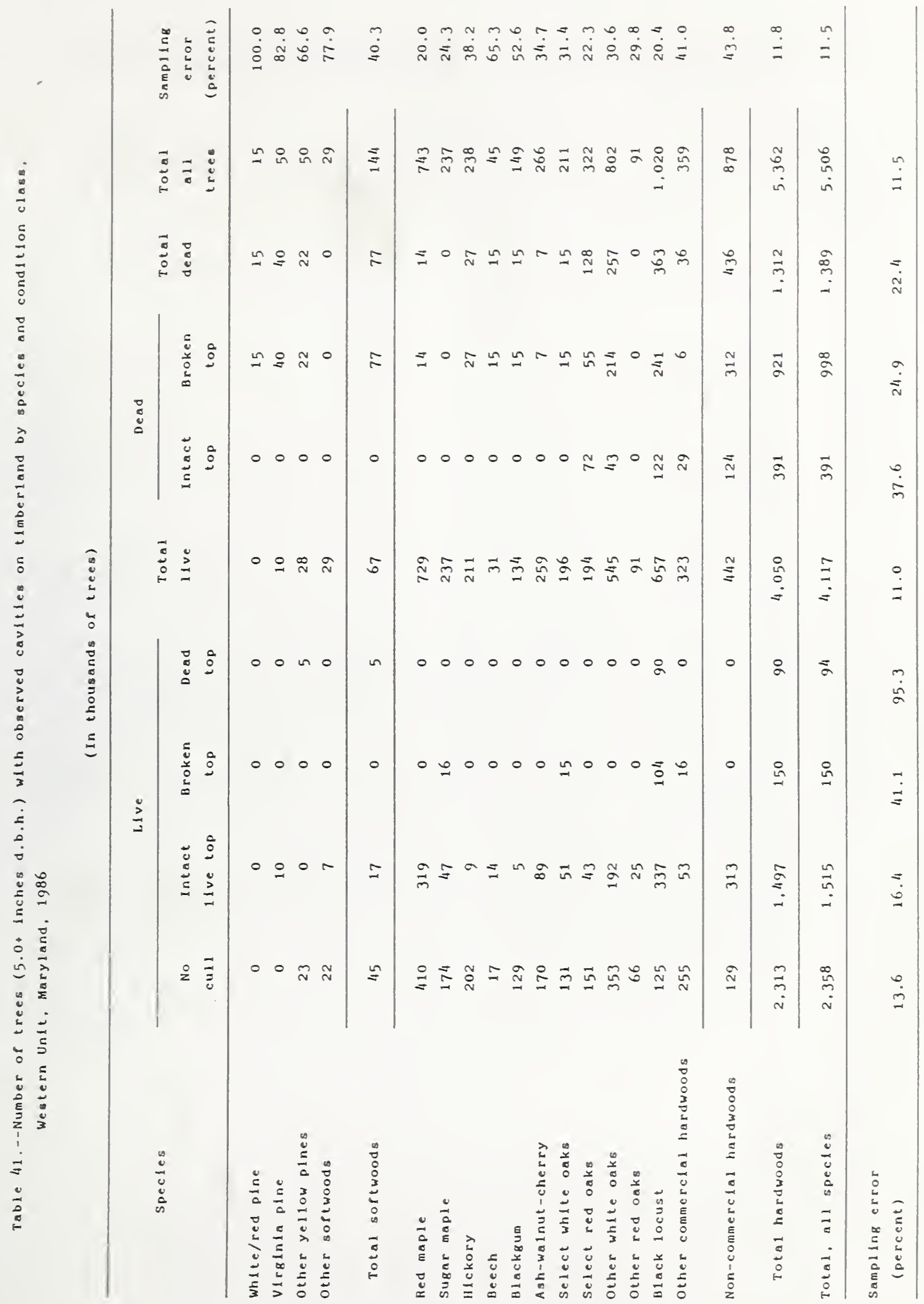




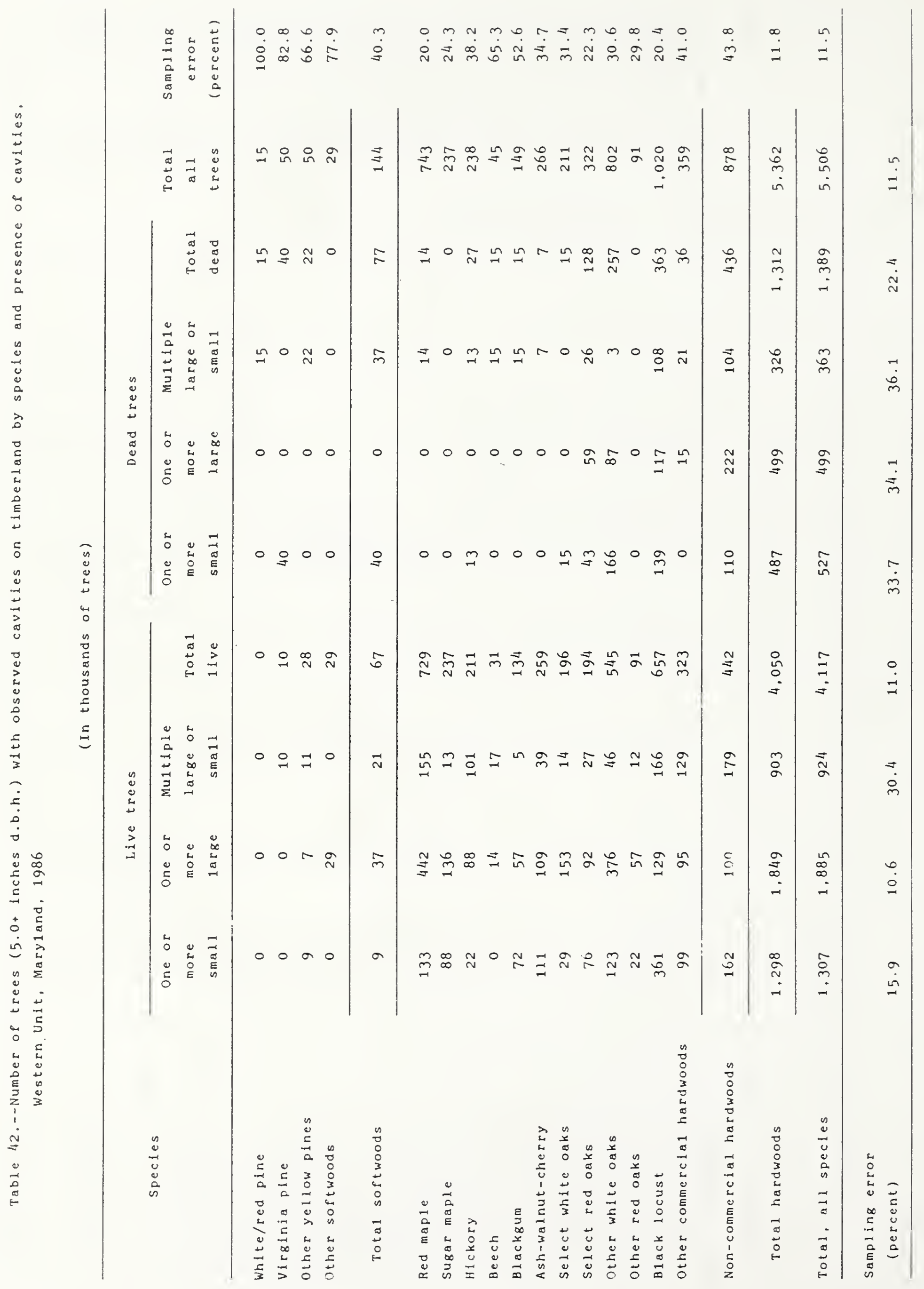


Table 43.--Number of seedlings, saplings, and shrubs on timberland by species and stand-size c1ass. Western Unit, Maryland, 1986

\begin{tabular}{|c|c|c|c|c|c|c|}
\hline \multirow{2}{*}{ Species } & \multicolumn{4}{|c|}{ Stand-size c10ss } & \multirow{2}{*}{$\begin{array}{c}\text { A.11 } \\
\text { c1asses }\end{array}$} & \multirow{2}{*}{$\begin{array}{l}\text { Samp11ng } \\
\text { error } \\
\text { (percent }\end{array}$} \\
\hline & Sawt $1 \mathrm{mber}$ & Polet lmber & Sap11ng and & Nonstocked & & \\
\hline Other pine species & 7 & 5 & 3 & 0 & 16 & $31 \cdot 7$ \\
\hline Other softwoods & 13 & 1 & 4 & 0 & 18 & $32 \cdot 3$ \\
\hline Total coniferous species & 20 & 6 & 7 & 0 & 34 & 21.9 \\
\hline Red map1e & 255 & 113 & 34 & 0 & 402 & 18.0 \\
\hline Sugar maple & 99 & 29 & 14 & 0 & 143 & 22.8 \\
\hline Other maple species & 89 & 18 & 1 & 0 & 109 & 27.6 \\
\hline serviceberry & 101 & 54 & 11 & 0 & 167 & 21.2 \\
\hline Azalea species & 10 & 4 & 1 & 0 & 15 & 35.1 \\
\hline Blrch spectes & 49 & 14 & 16 & 0 & 79 & 24.4 \\
\hline Hickory specles & 14 & 11 & 7 & 0 & 32 & $19 \cdot 5$ \\
\hline Dogwood species & 48 & 12 & 15 & 0 & 75 & 25.6 \\
\hline Amerlcan beech & 9 & 2 & 0 & 0 & 11 & 34.6 \\
\hline Ash species & 87 & 37 & 21 & 0 & 145 & 26.4 \\
\hline Huck1 eberry & 29 & 15 & 4 & 0 & 48 & 21.1 \\
\hline Witch-hazel & 34 & 12 & 3 & 0 & 49 & 14.9 \\
\hline Laure1 species & 9 & 7 & 2 & 0 & 19 & 34.2 \\
\hline Common spicebush & 3 & 0 & 0 & 0 & 3 & 58.4 \\
\hline Ye11ow-poplar & 7 & 0 & 0 & 0 & 7 & 67.0 \\
\hline Magnolia specles & 7 & 1 & 0 & 0 & 9 & $61 \cdot 3$ \\
\hline Tupe10 species & 17 & 11 & 0 & 0 & 28 & 33.0 \\
\hline Black cherry & 175 & 66 & 20 & 0 & 261 & $14 \cdot 3$ \\
\hline Other cherry species & 0 & 3 & 3 & 0 & 7 & $61 \cdot 5$ \\
\hline White oak & 66 & 28 & 25 & 0 & 119 & 28.5 \\
\hline Chestnut oak & 30 & 6 & 9 & 0 & 45 & 22.2 \\
\hline Northern red oak & 73 & 21 & 11 & 0 & 105 & $19 \cdot 3$ \\
\hline Other black oaks & 39 & 24 & 3 & 0 & 65 & $37 \cdot 7$ \\
\hline Rhododendron species & 17 & 1 & 0 & 0 & 17 & 43.0 \\
\hline Rose species & 2 & 1 & 0 & 0 & 3 & $50 \cdot 7$ \\
\hline Rubus species & 53 & 34 & 16 & 0 & 103 & 11.8 \\
\hline Sassafras & 119 & 67 & 23 & 0 & 209 & 24.0 \\
\hline B1 ueberry & 25 & 14 & 3 & 0 & 43 & 18.4 \\
\hline Elm species & 14 & 1 & 0 & 0 & 15 & 32.4 \\
\hline Maple-leaved viburnum & 8 & 2 & 3 & 0 & 13 & 30.8 \\
\hline Arrowwood & 3 & 2 & 0 & 0 & 5 & $54 \cdot 3$ \\
\hline Other viburnum species & 2 & 2 & 1 & 0 & 5 & 44.4 \\
\hline Other deciduous species & 122 & 68 & 55 & 0 & 245 & $17 \cdot 0$ \\
\hline Total deciduous species & 1.620 & 679 & 303 & 0 & 2,602 & 6.9 \\
\hline Unknown species & 18 & 9 & 4 & 0 & 31 & 24.9 \\
\hline Total, all species & 1.658 & 694 & 315 & 0 & 2,667 & 6.8 \\
\hline $\begin{array}{l}\text { Sampling error } \\
\text { (percent) }\end{array}$ & 10.9 & $17 \cdot 9$ & $34 \cdot 5$ & .0 & 6.8 & \\
\hline
\end{tabular}









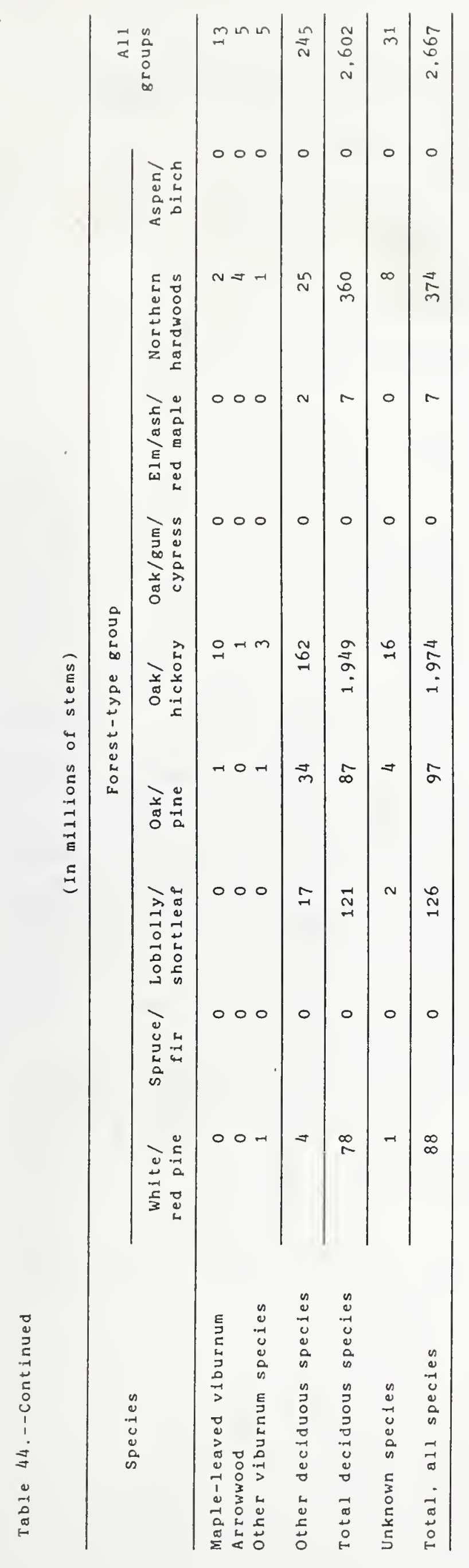


Table 45.--Number of seedlings, saplings, and shrubs on timberland by species and browse-utilization class, Western Unit, Maryland, 1986

(In millions of stems)

\begin{tabular}{|c|c|c|c|c|c|c|}
\hline \multirow{2}{*}{ Species } & \multicolumn{4}{|c|}{ Browse-utilization class } & \multirow{2}{*}{$\begin{array}{c}\text { All } \\
\text { classes }\end{array}$} & \multirow{2}{*}{$\begin{array}{c}\text { Sampling } \\
\text { error } \\
\text { (percent) }\end{array}$} \\
\hline & None & Light & Moderate & Heavy & & \\
\hline Other pine species & 16 & 0 & 0 & 0 & 16 & $31 \cdot 7$ \\
\hline Other softwoods & 18 & 0 & 0 & 0 & 18 & $32 \cdot 3$ \\
\hline Total coniferous species & 34 & 0 & 0 & 0 & 34 & $21 \cdot 9$ \\
\hline Red maple & 308 & 81 & 14 & 0 & 402 & 18.0 \\
\hline Sugar maple & 102 & 35 & 6 & 0 & 143 & 22.8 \\
\hline Other maple species & 103 & 3 & 0 & 2 & 109 & 27.6 \\
\hline Serviceberry & 145 & 19 & 2 & 1 & 167 & 21.2 \\
\hline Azalea species & 15 & 0 & 0 & 0 & 15 & 35.1 \\
\hline Birch species & 67 & 5 & 7 & 0 & 79 & 24.4 \\
\hline Hickory species & 31 & 1 & 1 & 0 & 32 & 19.5 \\
\hline American beech & 8 & 3 & 0 & 0 & 11 & 34.6 \\
\hline Ash species & 118 & 21 & 5 & 0 & 145 & 26.4 \\
\hline Huckleberry & 41 & 7 & 0 & 0 & 48 & 21.1 \\
\hline Witch-haze 1 & 41 & 6 & 2 & 0 & 49 & 14.9 \\
\hline Laurel species & 18 & 0 & 1 & 0 & 19 & 34.2 \\
\hline Common splcebush & 3 & 1 & 0 & 0 & 3 & 58.4 \\
\hline Yellow-poplar & 7 & 0 & 0 & 0 & 7 & 67.0 \\
\hline Magnolia species & 9 & 0 & 0 & 0 & 9 & $61 \cdot 3$ \\
\hline Tupelo species & 25 & 0 & 1 & 1 & 28 & 33.0 \\
\hline Black cherry & 184 & 69 & 7 & 0 & 261 & $14 \cdot 3$ \\
\hline Other cherry species & 7 & 0 & 0 & 0 & 7 & 61.5 \\
\hline White oak & 113 & 4 & 1 & 0 & 119 & 28.5 \\
\hline Chestnut oak & 34 & 7 & 3 & 0 & 45 & $22 \cdot 2$ \\
\hline Northern red oak & 86 & 16 & 4 & 0 & 105 & $19 \cdot 3$ \\
\hline Other black oaks & 57 & 7 & 2 & 0 & 65 & $37 \cdot 7$ \\
\hline Rhododendron species & 17 & 0 & 0 & 0 & 17 & 43.0 \\
\hline Sassafras & 164 & 34 & 12 & 0 & 209 & 24.0 \\
\hline Blueberry & 37 & 5 & 1 & 0 & 43 & 18.4 \\
\hline Elm species & 14 & 0 & 1 & 0 & 15 & 32.4 \\
\hline Maple-leaved viburnum & 7 & 3 & 2 & 1 & 13 & 30.8 \\
\hline Arrowwood & 3 & 1 & 1 & 0 & 5 & $54 \cdot 3$ \\
\hline Other viburnum species & 5 & 1 & 0 & 0 & 5 & 44.4 \\
\hline Other deciduous species & 169 & 73 & 2 & 2 & 245 & 17.0 \\
\hline Total deciduous species & 2,104 & 416 & 75 & 7 & 2.602 & 6.9 \\
\hline Unknown species & 28 & 1 & 2 & 0 & 31 & 24.9 \\
\hline Total, all species & 2.166 & 417 & 76 & 7 & 2.667 & 6.8 \\
\hline Sampling error & & & & & & \\
\hline$($ percent $)$ & $7 \cdot 9$ & 16.5 & 19.9 & $42 \cdot 3$ & 6.8 & \\
\hline
\end{tabular}




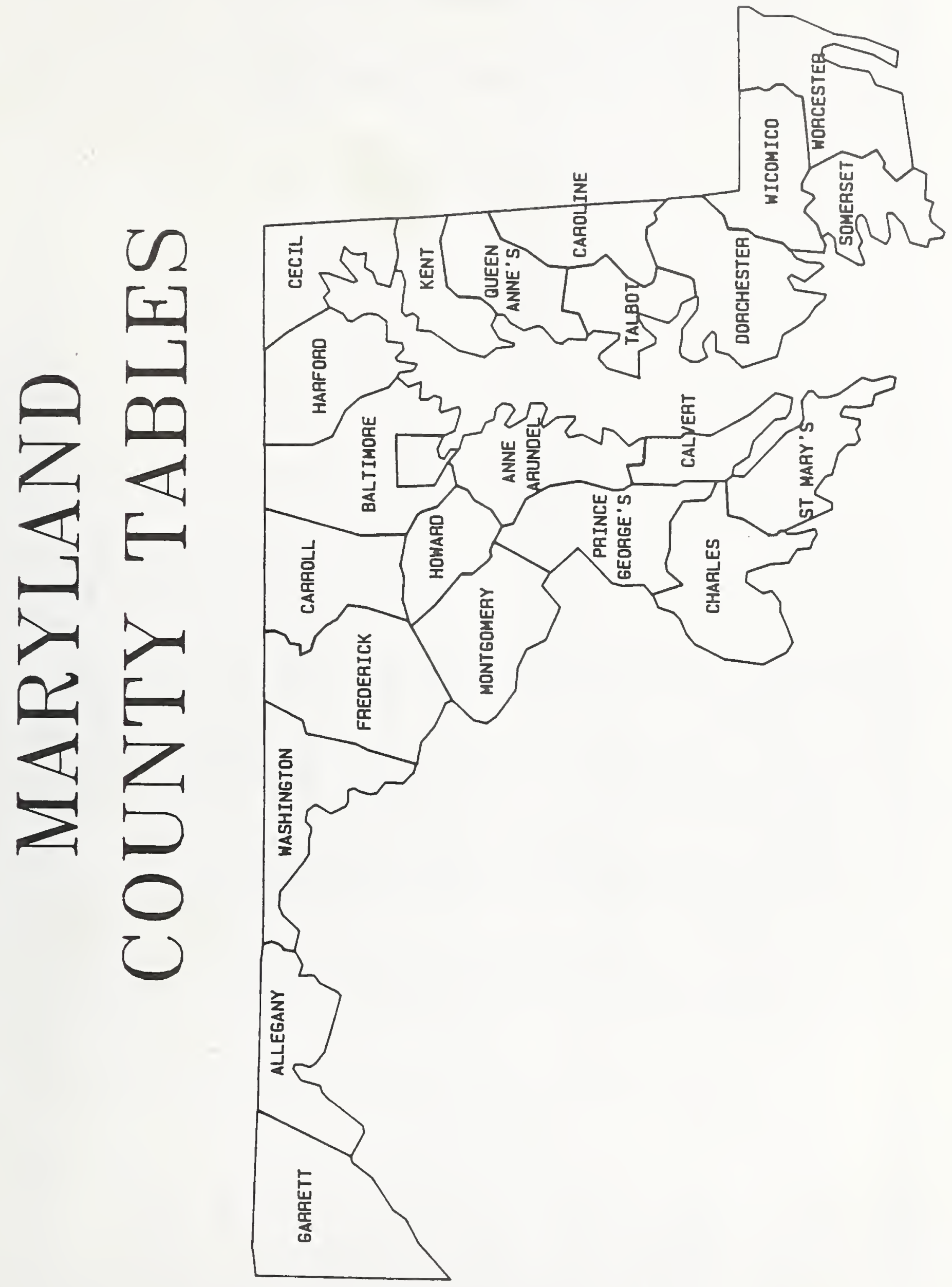




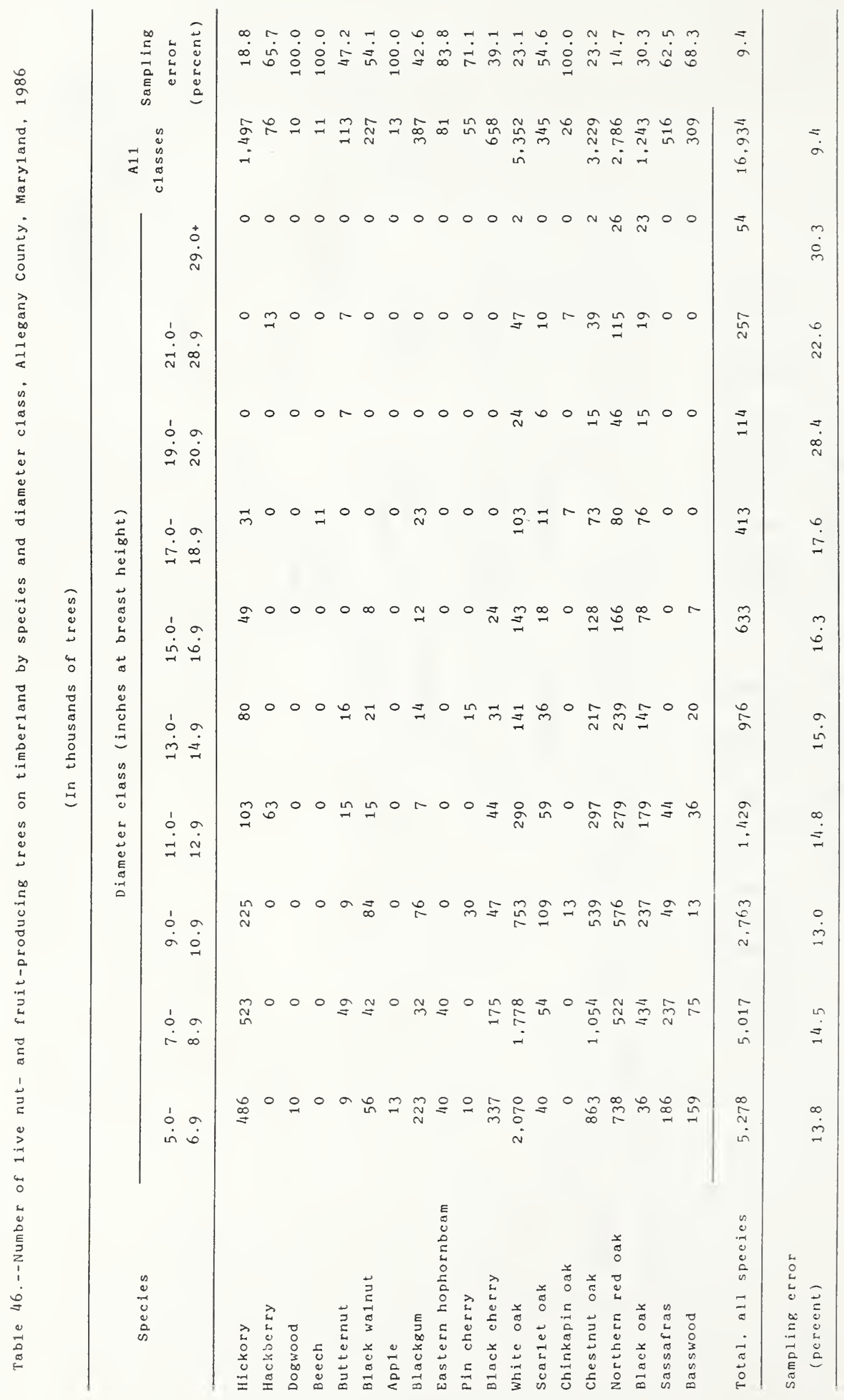




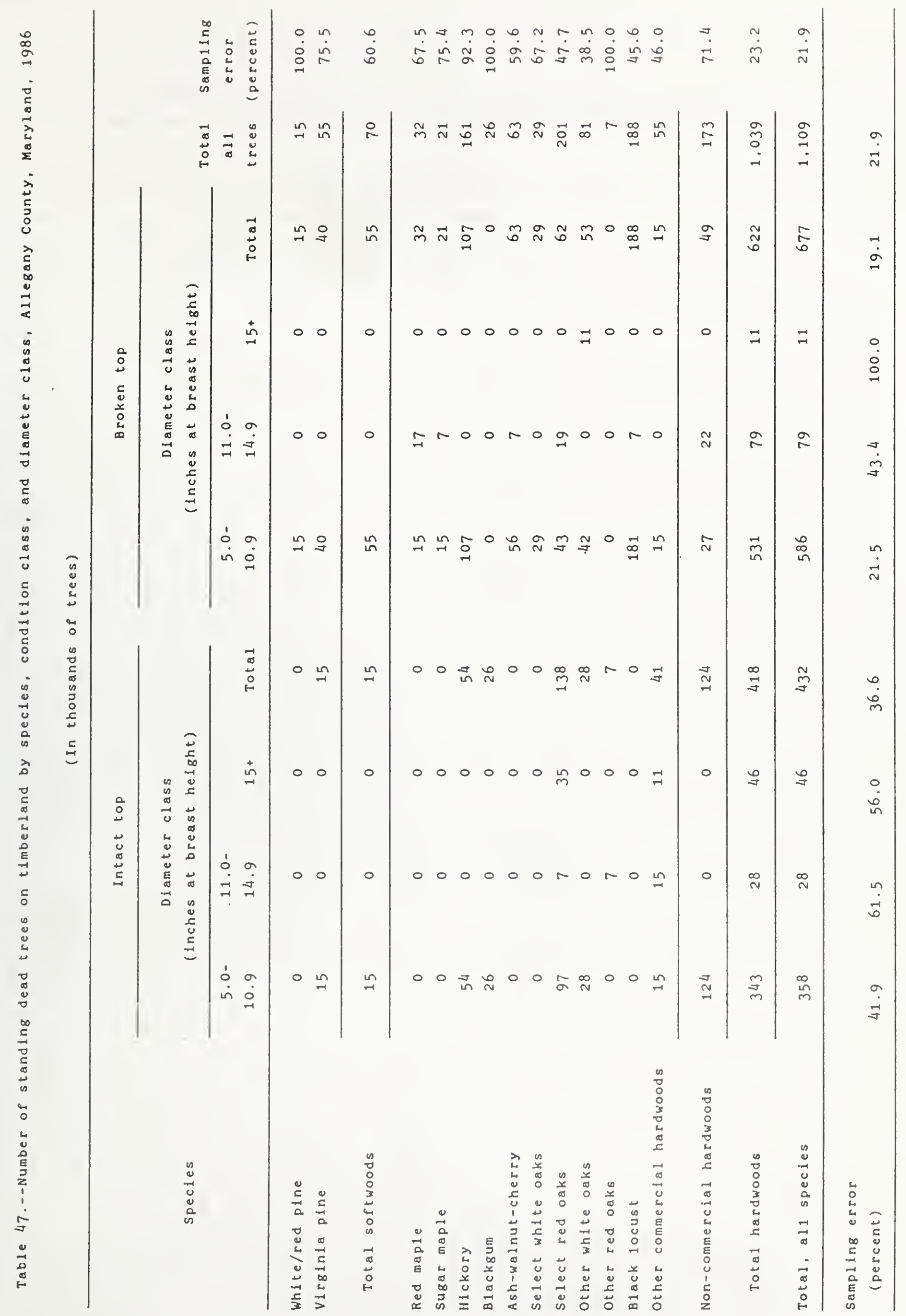


Table 48.--Number of seedlings, saplings, and shrubs on timberland by species and browse-utilization class. Allegany County, Maryland, 1986

(In milions of stems)

\begin{tabular}{|c|c|c|c|c|c|c|}
\hline \multirow{2}{*}{ species } & \multicolumn{4}{|c|}{ Browse-utilization class } & \multirow{2}{*}{$\begin{array}{c}\text { All } \\
\text { classes }\end{array}$} & \multirow{2}{*}{$\begin{array}{c}\text { Sampling } \\
\text { error } \\
\text { (percent) }\end{array}$} \\
\hline & None & Light & Moderate & Heavy & & \\
\hline Other pine species & 9 & 0 & 0 & 0 & 9 & 43.1 \\
\hline Other softwoods & 3 & 0 & 0 & 0 & 3 & 86.9 \\
\hline Total coniferous species & 13 & 0 & 0 & 0 & 13 & 37.2 \\
\hline Red maple & 51 & 22 & 2 & 0 & 75 & 28.8 \\
\hline Sugar maple & 47 & 6 & 0 & 0 & 52 & 27.4 \\
\hline Other maple species & 13 & 1 & 0 & 0 & 15 & 42.8 \\
\hline Serviceberry & 60 & 4 & 0 & 1 & 65 & 30.6 \\
\hline Azalea species & 4 & 0 & 0 & 0 & 4 & 63.7 \\
\hline Birch species & 5 & 2 & 3 & 0 & 10 & 56.9 \\
\hline Hickory species & 17 & 0 & 1 & 0 & 18 & 22.4 \\
\hline Dogwood species & 58 & 2 & 0 & 0 & 59 & 30.9 \\
\hline Ash species & 66 & 11 & 3 & 0 & 80 & 29.6 \\
\hline Huckleberry & 10 & 4 & 0 & 0 & 14 & 30.7 \\
\hline Witch-hazel & 7 & 1 & 0 & 0 & 9 & $31 \cdot 5$ \\
\hline Common spicebush & 3 & 1 & 0 & 0 & 3 & 58.4 \\
\hline Yellow-poplar & 2 & 0 & 0 & 0 & 2 & 100.0 \\
\hline Magnolia species & 1 & 0 & 0 & 0 & 1 & 100.0 \\
\hline Tupelo species & 7 & 0 & 0 & 0 & 7 & 41.2 \\
\hline Black cherry & 17 & 6 & 4 & 0 & 27 & 33.1 \\
\hline Other cherry species & 1 & 0 & 0 & 0 & 1 & 71.9 \\
\hline White oak & 31 & 1 & 0 & 0 & 33 & 40.8 \\
\hline Chestnut oak & 18 & 6 & 0 & 0 & 24 & 29.6 \\
\hline Northern red oak & 37 & 10 & 0 & 0 & 47 & $25 \cdot 5$ \\
\hline Other black oaks & 46 & 4 & 2 & 0 & 53 & 45.6 \\
\hline Rose species & 0 & 1 & 0 & 0 & 1 & 72.1 \\
\hline Rubus species & 23 & 5 & 0 & 0 & 27 & 22.8 \\
\hline Sassafras & 42 & 12 & 3 & 0 & 57 & 36.8 \\
\hline Blueberry & 27 & 4 & 0 & 0 & 31 & 20.9 \\
\hline Elm species & 10 & 0 & 1 & 0 & 11 & $35 \cdot 3$ \\
\hline Maple-leaved viburnum & 3 & 1 & 1 & 1 & 5 & 37.2 \\
\hline Other viburnum species & 2 & 0 & 0 & 0 & 2 & 63.7 \\
\hline Other deciduous species & 73 & 24 & 2 & 0 & 99 & 23.4 \\
\hline Total deciduous species & 684 & 129 & 22 & 1 & 836 & $9 \cdot 5$ \\
\hline Unknown species & 6 & 1 & 1 & 0 & 8 & 32.9 \\
\hline Total, all species & 702 & 130 & 23 & 1 & 857 & 9.4 \\
\hline $\begin{array}{l}\text { Sampling er:tr } \\
\text { (percent) }\end{array}$ & 11.0 & 19.2 & 30.8 & 68.0 & 9.4 & \\
\hline
\end{tabular}




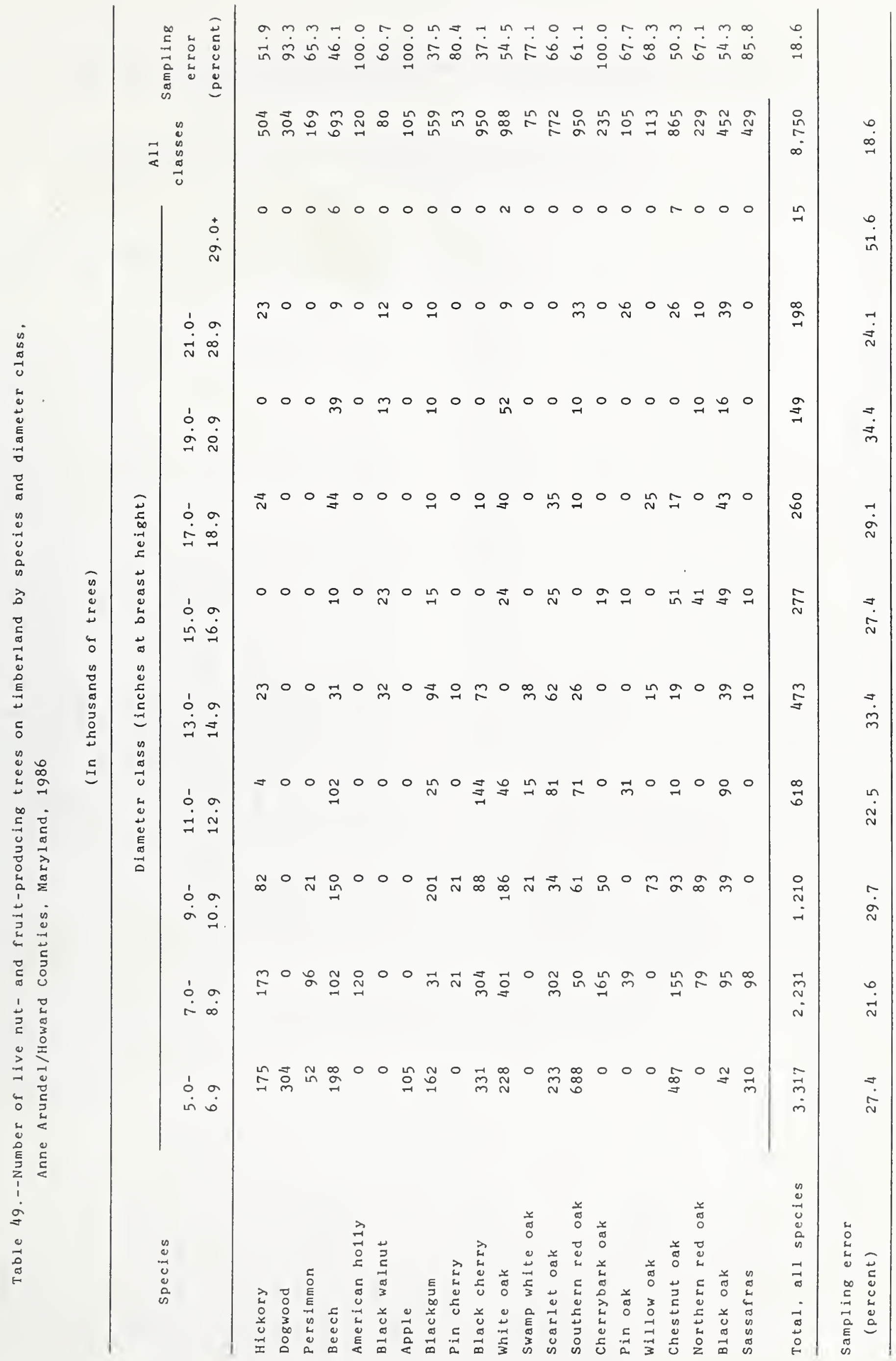




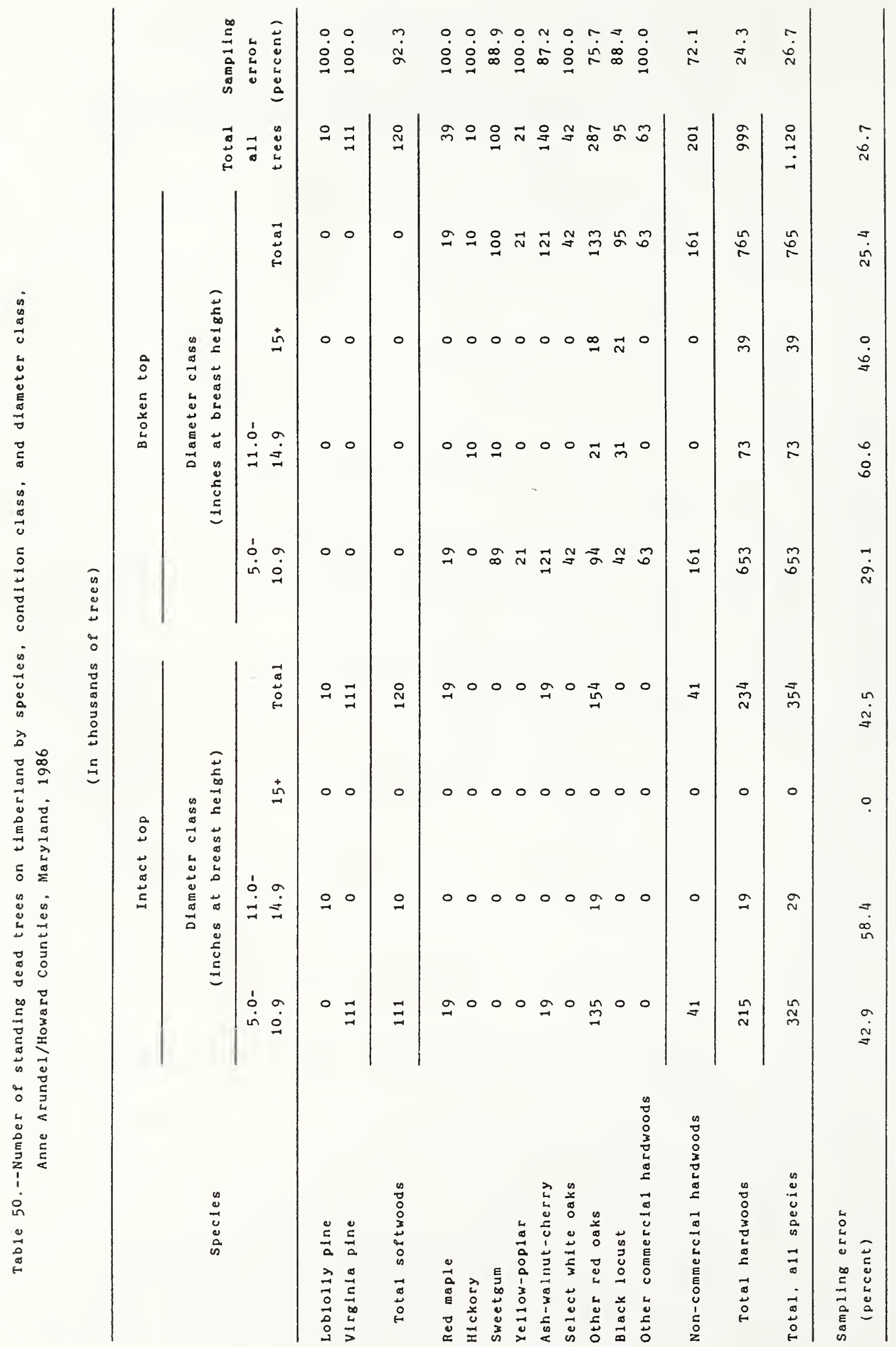


Table 51.--Number of seedlings, saplings, and shrubs on timberland by species and browse-utilization class. Anne Arundel/Howard Counties. Maryland, 1986

( In millions of stems)

\begin{tabular}{|c|c|c|c|c|c|c|}
\hline \multirow{2}{*}{ Species } & \multicolumn{4}{|c|}{ Browse-ut111zation class } & \multirow{2}{*}{$\begin{array}{c}\text { All } \\
\text { classes }\end{array}$} & \multirow{2}{*}{$\begin{array}{c}\text { Sampling } \\
\text { error } \\
\text { (percont) }\end{array}$} \\
\hline & None & LIght & Moderate & Heavy & & \\
\hline Loblolly pine & 1 & 0 & 0 & 0 & 1 & 100.0 \\
\hline other pine species & 9 & 0 & 0 & 0 & 9 & 48.6 \\
\hline Total coniferous species & 10 & 0 & 0 & 0 & 10 & 44.9 \\
\hline Red maple & 24 & 0 & 0 & 0 & 24 & 45.4 \\
\hline Other maple species & 9 & 0 & 0 & 0 & 9 & 83.6 \\
\hline Alder species & 4 & 0 & 0 & 0 & 4 & 71.6 \\
\hline Serviceberry & 2 & 0 & 0 & 0 & 2 & 70.7 \\
\hline Azálea species & 0 & 0 & 0 & 0 & 0 & 100.0 \\
\hline Common pawpaw & 1 & 0 & 0 & 0 & 1 & 100.0 \\
\hline Birch species & 2 & 0 & 0 & 0 & 2 & 78.0 \\
\hline Hickory species & 6 & 0 & 0 & 0 & 6 & 60.2 \\
\hline Dogwood species & 29 & 0 & 0 & 0 & 29 & 41.2 \\
\hline American beech & 10 & 0 & 0 & 0 & 10 & 80.4 \\
\hline Ash species & 9 & 0 & 0 & 0 & 9 & 83.5 \\
\hline American holly & 15 & 0 & 0 & 0 & 15 & 83.2 \\
\hline Laurel species & 3 & 0 & 0 & 0 & 3 & 88.4 \\
\hline Common splcebush & 8 & 0 & 0 & 0 & 8 & 57.8 \\
\hline Sweetgum & 58 & 0 & 0 & 0 & 58 & 40.3 \\
\hline Yellow-poplar & 3 & 0 & 0 & 0 & 3 & $71 \cdot 9$ \\
\hline Tupelo species & 31 & 0 & 3 & 0 & 34 & 46.6 \\
\hline Black cherry & 30 & 0 & 0 & 0 & 30 & 43.0 \\
\hline Other cherry species & 8 & 0 & 0 & 0 & 8 & $77 \cdot 7$ \\
\hline White oak & 10 & 0 & 0 & 0 & 10 & 40.4 \\
\hline Chestnut oak & 2 & 0 & 0 & 0 & 2 & 70.7 \\
\hline Northern red oak & 1 & 0 & 0 & 0 & 1 & $76 \cdot 5$ \\
\hline other black oaks & 22 & 1 & 0 & 0 & 23 & $34 \cdot 5$ \\
\hline Rhododendron specles & 1 & 0 & 0 & 0 & 1 & 100.0 \\
\hline Rose species & 5 & 0 & 0 & 0 & 5 & 61.8 \\
\hline Rubus species & 24 & 0 & 0 & 0 & 24 & $27 \cdot 7$ \\
\hline Sassafras & 12 & 0 & 0 & 0 & 12 & 51.2 \\
\hline Blueberry & 25 & 0 & 0 & 0 & 25 & 36.8 \\
\hline Elm species & 1 & 0 & 0 & 0 & 1 & 100.0 \\
\hline Maple-leaved viburnum & 2 & 0 & 0 & 0 & 2 & 66.8 \\
\hline Arrowwood & 12 & 0 & 0 & 0 & 12 & 36.7 \\
\hline Other viburnum species & 3 & 0 & 0 & 0 & 3 & $71 \cdot 2$ \\
\hline other deciduous species & 25 & 0 & 0 & 0 & 25 & $1: 0.7$ \\
\hline Total deciduous species & 400 & 1 & 3 & 0 & 404 & 12.8 \\
\hline Unknown species & 20 & 0 & 0 & 0 & 20 & 48.9 \\
\hline Total, all species & 431 & 1 & 3 & 0 & 435 & $13 \cdot 3$ \\
\hline $\begin{array}{l}\text { Sampling error } \\
\text { (percent) }\end{array}$ & & & & & & \\
\hline & 13.4 & 100.0 & 100.0 & .0 & $13 \cdot 3$ & \\
\hline
\end{tabular}




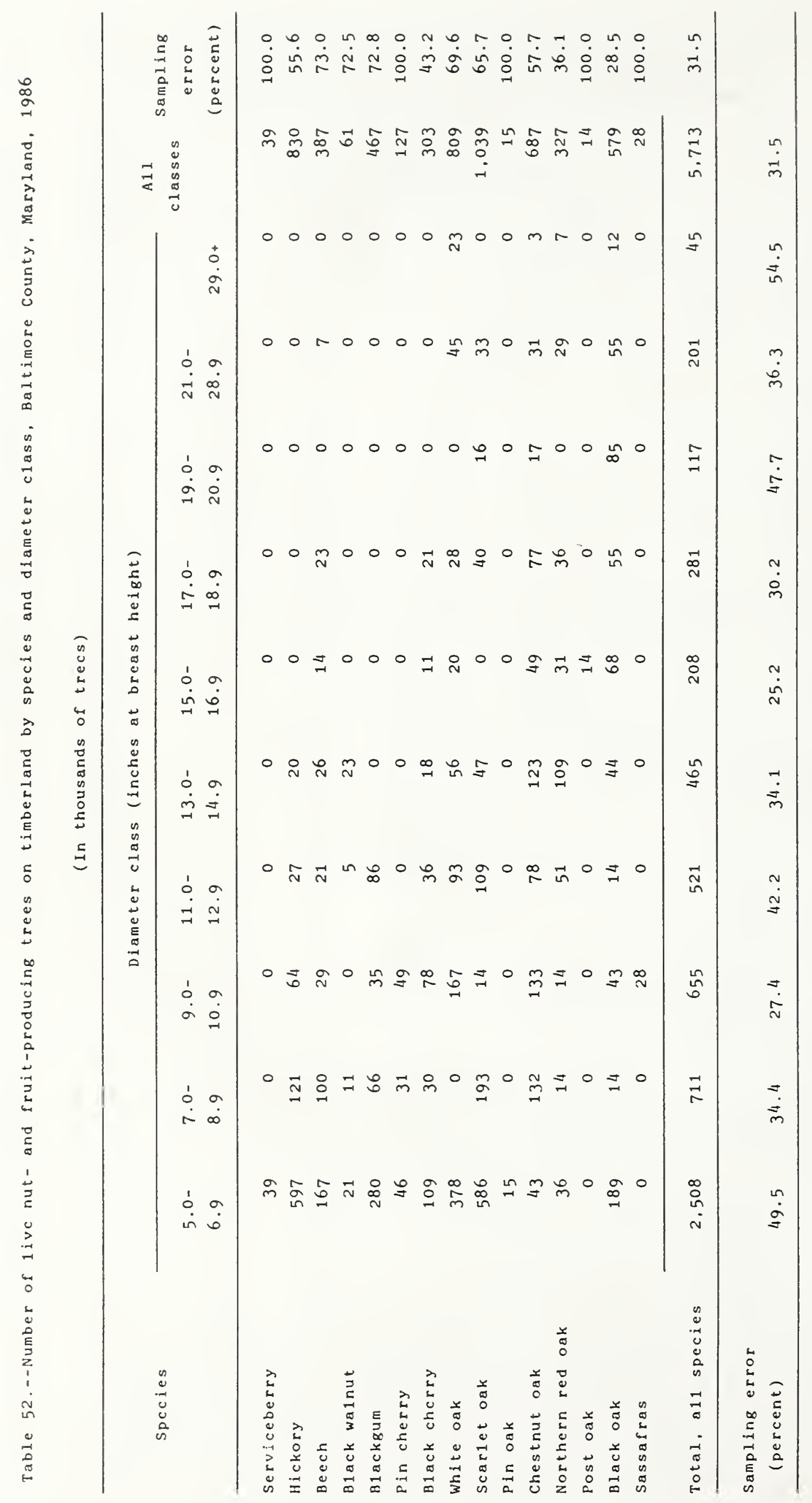




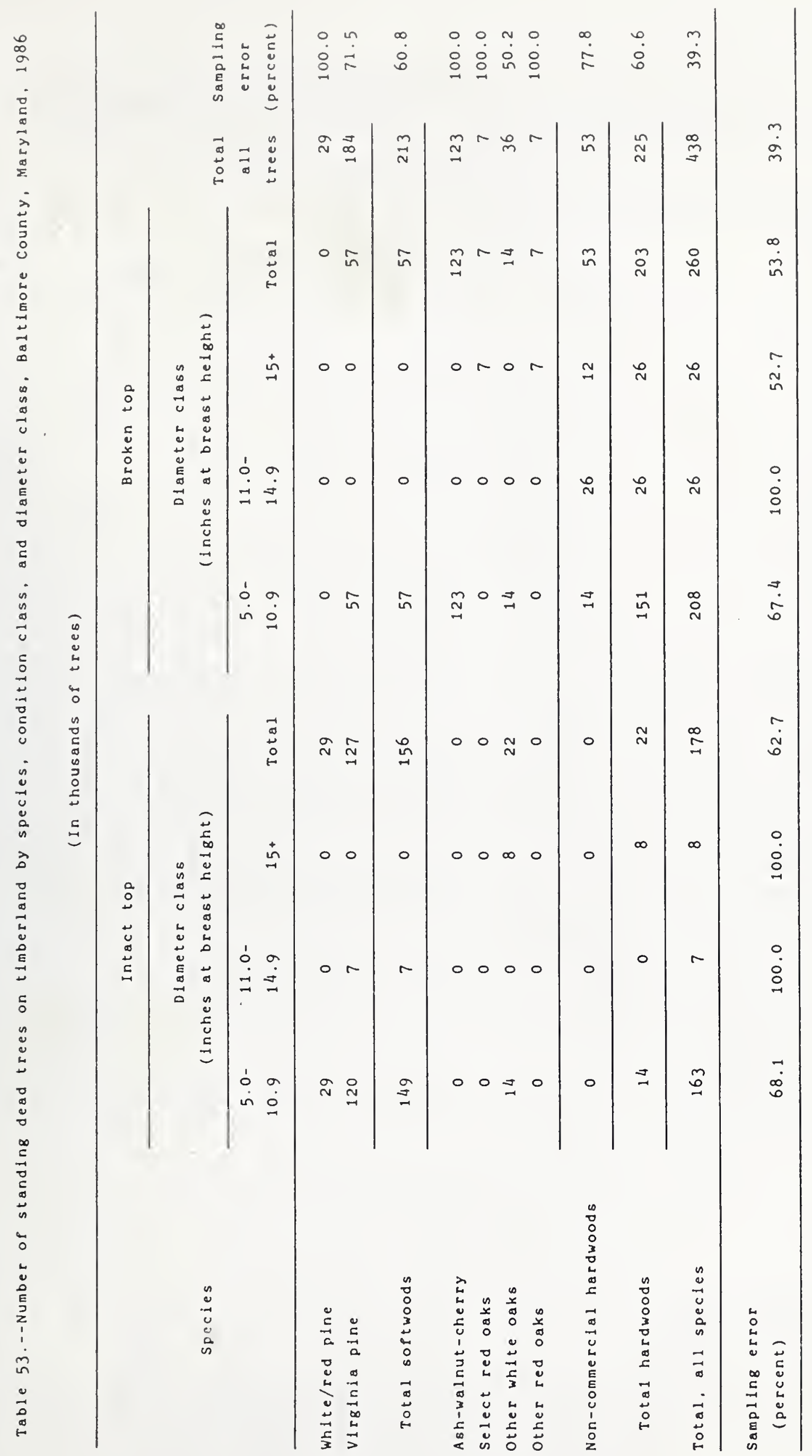


Table 54.--Number of seedlings, saplings, and shrubs on timberland by species and browse-ut111zation class. Baltimore County, Maryland, 1986

(In millions of stems)

\begin{tabular}{|c|c|c|c|c|c|c|}
\hline \multirow{2}{*}{ Spec1es } & \multicolumn{4}{|c|}{ Browse-ut 111 zation class } & \multirow{2}{*}{$\begin{array}{c}\text { A } 11 \\
\text { c lasses }\end{array}$} & \multirow{2}{*}{$\begin{array}{c}\text { Sampling } \\
\text { error } \\
\text { (percent) }\end{array}$} \\
\hline & None & L $1 \mathrm{ght}$ & Moderate & Heavy & & \\
\hline other pine species & 1 & 0 & 0 & 0 & 1 & 100.0 \\
\hline Total conflerous species & 1 & 0 & 0 & 0 & 1 & 100.0 \\
\hline Red maple & 32 & 22 & 0 & 0 & 54 & 43.6 \\
\hline Serviceberry & 10 & 0 & 0 & 0 & 10 & 100.0 \\
\hline Azalea species & 4 & 0 & 0 & 0 & 4 & 52.0 \\
\hline Hickory species & 23 & 2 & 1 & 0 & 25 & 42.2 \\
\hline Dogwood species & 33 & 7 & 7 & 0 & 46 & 25.0 \\
\hline American beech & 6 & 0 & 0 & 0 & 6 & $57 \cdot 2$ \\
\hline Ash species & 1 & 0 & 0 & 0 & 1 & 100.0 \\
\hline Witch-hazel & 1 & 0 & 0 & 0 & 1 & 100.0 \\
\hline Laurel species & 4 & 0 & 0 & 0 & 4 & 54.8 \\
\hline Common splcebush & 9 & 1 & 3 & 2 & 15 & 40.3 \\
\hline Sweetgum & 2 & 0 & 0 & 0 & 2 & 100.0 \\
\hline Yellow-poplar & 18 & 9 & 0 & 0 & 26 & 51.6 \\
\hline Tupelo species & 43 & 21 & 0 & 0 & 64 & $52 \cdot 2$ \\
\hline Black cherry & 64 & 11 & 3 & 0 & 78 & 59.4 \\
\hline White oak & 6 & 0 & 0 & 0 & 6 & 74.1 \\
\hline Chestnut oak & 8 & 0 & 1 & 0 & 9 & 82.3 \\
\hline Northern red oak & 6 & 1 & 0 & 0 & 7 & 92.1 \\
\hline other black oaks & 5 & 0 & 0 & 0 & 5 & $58 \cdot 5$ \\
\hline Rose spectes & 7 & 1 & 0 & 0 & 8 & 54.0 \\
\hline Rubus species & 24 & 1 & 0 & 0 & 25 & 27.0 \\
\hline Sassafras & 23 & 10 & 1 & 0 & 34 & $40 \cdot 3$ \\
\hline Blueberry & 9 & 3 & 1 & 0 & 13 & $39 \cdot 3$ \\
\hline Elm species & 10 & 0 & 1 & 0 & 12 & 62.1 \\
\hline Maple-leaved viburnum & 9 & 4 & 0 & 0 & 13 & 44.0 \\
\hline Arrowwood & 7 & 0 & 0 & 0 & 7 & 42.4 \\
\hline other viburnum specles & 2 & 2 & 0 & 0 & 4 & 62.0 \\
\hline Other deciduous specles & 9 & 1 & 0 & 0 & 11 & 41.6 \\
\hline Total deciduous spectes & 377 & 93 & 17 & 2 & 489 & 29.2 \\
\hline Unknown species & 1 & 0 & 0 & 0 & 1 & 100.0 \\
\hline Total. all species & 378 & 93 & 17 & 2 & 491 & 29.1 \\
\hline Sampling error & & & & & & \\
\hline (percent) & $27 \cdot 7$ & $51 \cdot 1$ & $50 \cdot 2$ & 100.0 & 29.1 & \\
\hline
\end{tabular}




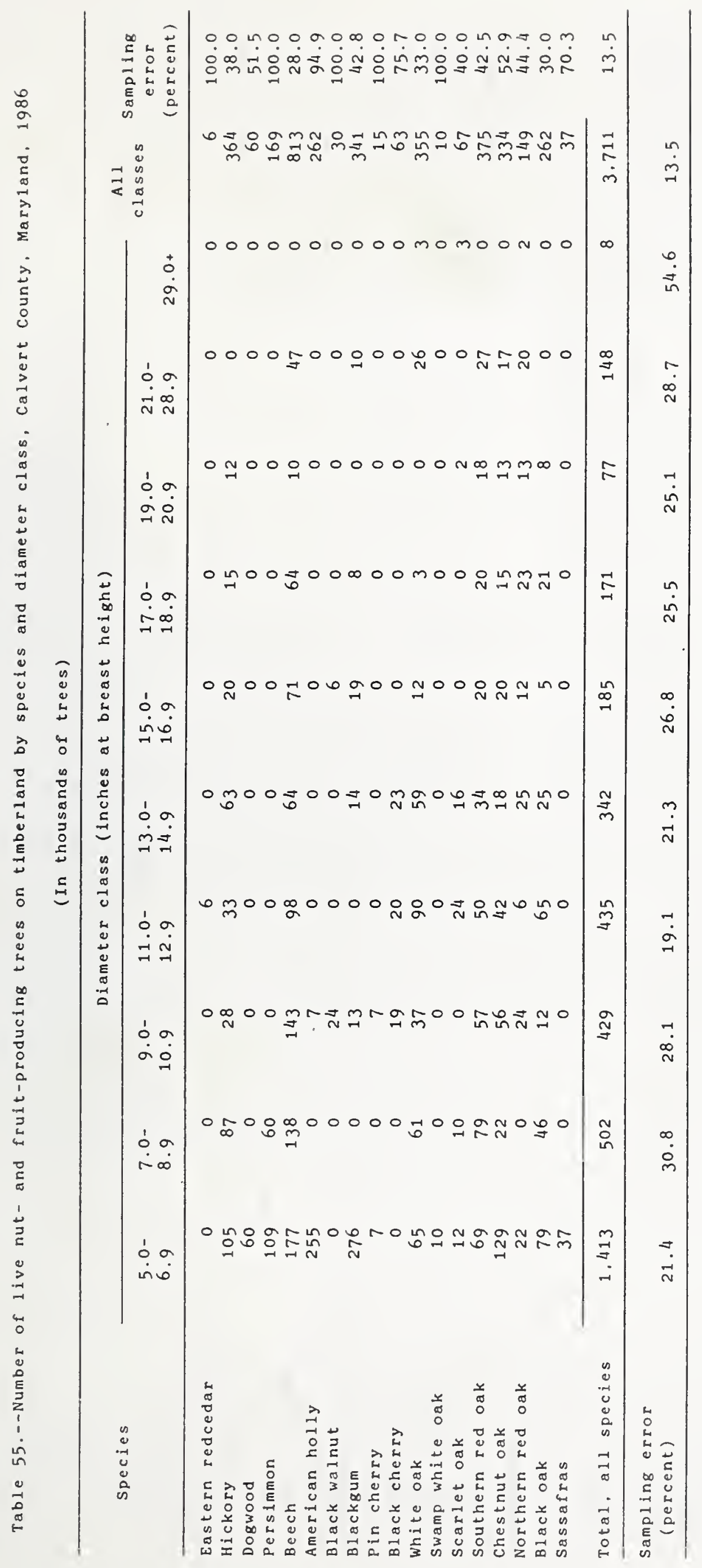




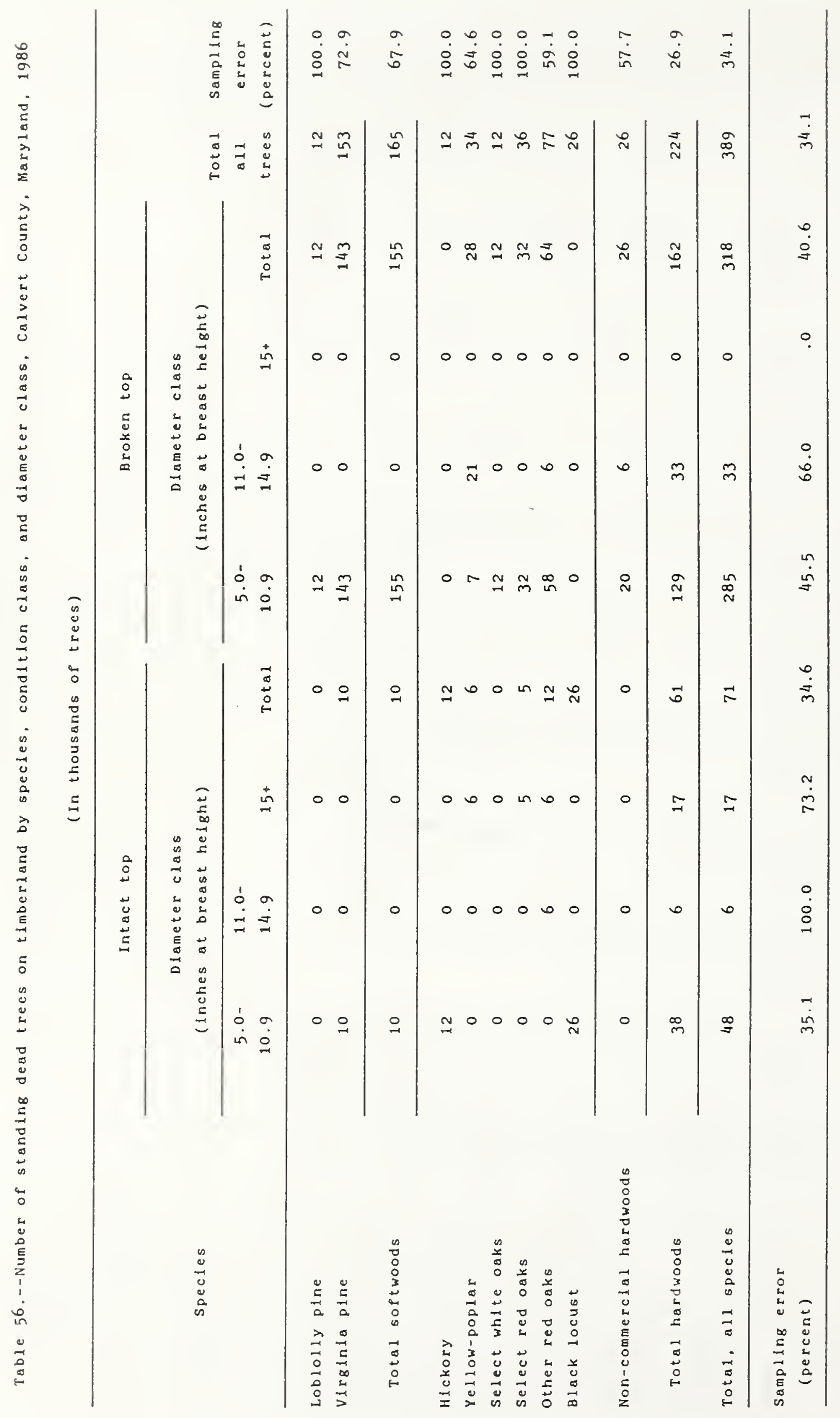


Table 57.--Number of seedlings, saplings, and shrubs on timberland by species and browse-utilization class, Calvert County, Maryland, 1986

(In mil11ons of stems)

\begin{tabular}{|c|c|c|c|c|c|c|}
\hline \multirow{2}{*}{ Species } & \multicolumn{4}{|c|}{ Browse-utilization class } & \multirow{2}{*}{$\begin{array}{c}\text { Al1 } \\
\text { classes }\end{array}$} & \multirow{2}{*}{$\begin{array}{c}\text { Sampling } \\
\text { error } \\
\text { (percent) }\end{array}$} \\
\hline & None & Light & Moderate & He avy & & \\
\hline Loblolly pine & 1 & 0 & 0 & 0 & 1 & 100.0 \\
\hline Other pine species & 1 & 0 & 0 & 0 & 1 & $81 \cdot 3$ \\
\hline Total coniferous species & 1 & 0 & 0 & 0 & 1 & $60 \cdot 9$ \\
\hline Red maple & 27 & 0 & 0 & 0 & 27 & 26.5 \\
\hline A1der species & 1 & 0 & 0 & 0 & 1 & $71 \cdot 2$ \\
\hline Common pawpaw & 81 & 9 & 0 & 0 & 90 & 35.8 \\
\hline Birch species & 1 & 0 & 0 & 0 & 1 & 100.0 \\
\hline Hickory species & 10 & 0 & 0 & 0 & 10 & $36 \cdot 5$ \\
\hline Dogwood species & 25 & 0 & 0 & 0 & 25 & 32.8 \\
\hline American beech & 14 & 1 & 0 & 1 & 16 & 28.0 \\
\hline Ash species & 0 & 0 & 0 & 0 & 0 & 100.0 \\
\hline American ho11y & 31 & 0 & 0 & 0 & 31 & 29.2 \\
\hline Laurel species & 9 & 0 & 0 & 0 & 9 & 32.0 \\
\hline Common splcebush & 8 & 0 & 0 & 0 & 8 & 33.6 \\
\hline Swee tgum & 10 & 0 & 0 & 0 & 10 & 53.0 \\
\hline Yellow-pop1ar & 4 & 0 & 0 & 0 & 4 & 44.1 \\
\hline Tupelo species & 6 & 1 & 0 & 0 & 7 & 40.4 \\
\hline B1ack cherry & 3 & 0 & 0 & 0 & 3 & 50.1 \\
\hline Other cherry species & 1 & 1 & 0 & 0 & 2 & $61 \cdot 3$ \\
\hline White oak & 6 & 0 & 0 & 0 & 6 & $51 \cdot 6$ \\
\hline Chestnut oak & 1 & 0 & 0 & 0 & $\cdot 1$ & 100.0 \\
\hline Northern red oak & 1 & 0 & 0 & 0 & 1 & $86 \cdot 3$ \\
\hline Other black oaks & 7 & 0 & 0 & 0 & 7 & $52 \cdot 4$ \\
\hline Rubus species & 5 & 0 & 0 & 0 & 5 & $35 \cdot 3$ \\
\hline Sassafras & 6 & 4 & 0 & 0 & 10 & 47.0 \\
\hline Blueberry & 8 & 1 & 0 & 0 & 9 & $27 \cdot 9$ \\
\hline Elm species & 1 & 0 & 0 & 0 & 1 & 100.0 \\
\hline Maple-leaved viburnum & 9 & 0 & 0 & 0 & 9 & $37 \cdot 0$ \\
\hline Arrowwood & 1 & 0 & 0 & 0 & 1 & 100.0 \\
\hline Other deciduous species & 9 & 0 & 0 & 0 & 9 & $33 \cdot 2$ \\
\hline Total deciduous species & 287 & 16 & 0 & 1 & 303 & 13.0 \\
\hline Unknown species & 1 & 0 & 0 & 0 & 1 & 100.0 \\
\hline Tota1, all species & 289 & 16 & 0 & 1 & 305 & $12 \cdot 9$ \\
\hline Sampling e ror & & & & & & \\
\hline$($ percent) & $13 \cdot 5$ & 57.6 & .0 & 100.0 & $12 \cdot 9$ & \\
\hline
\end{tabular}




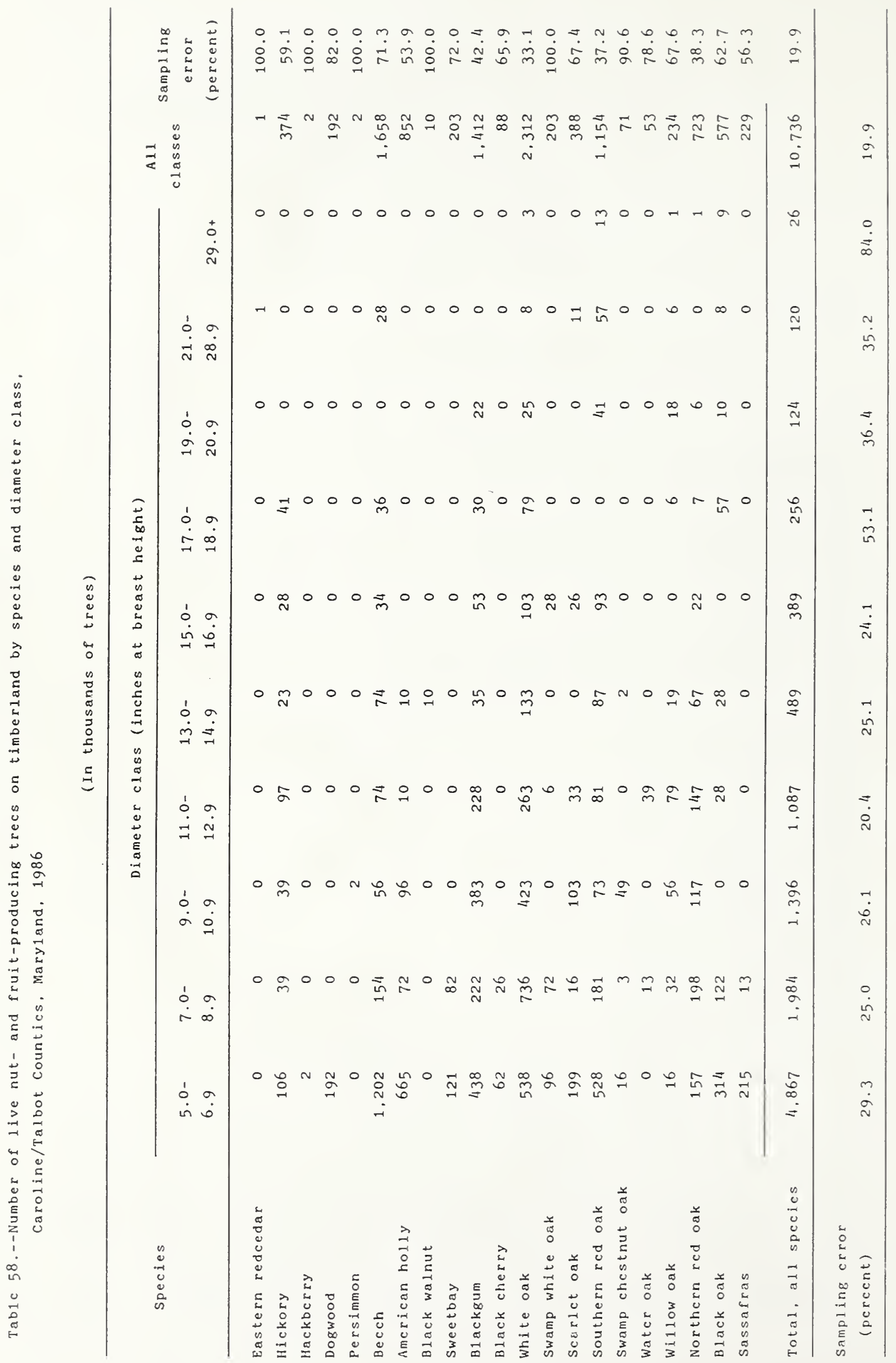




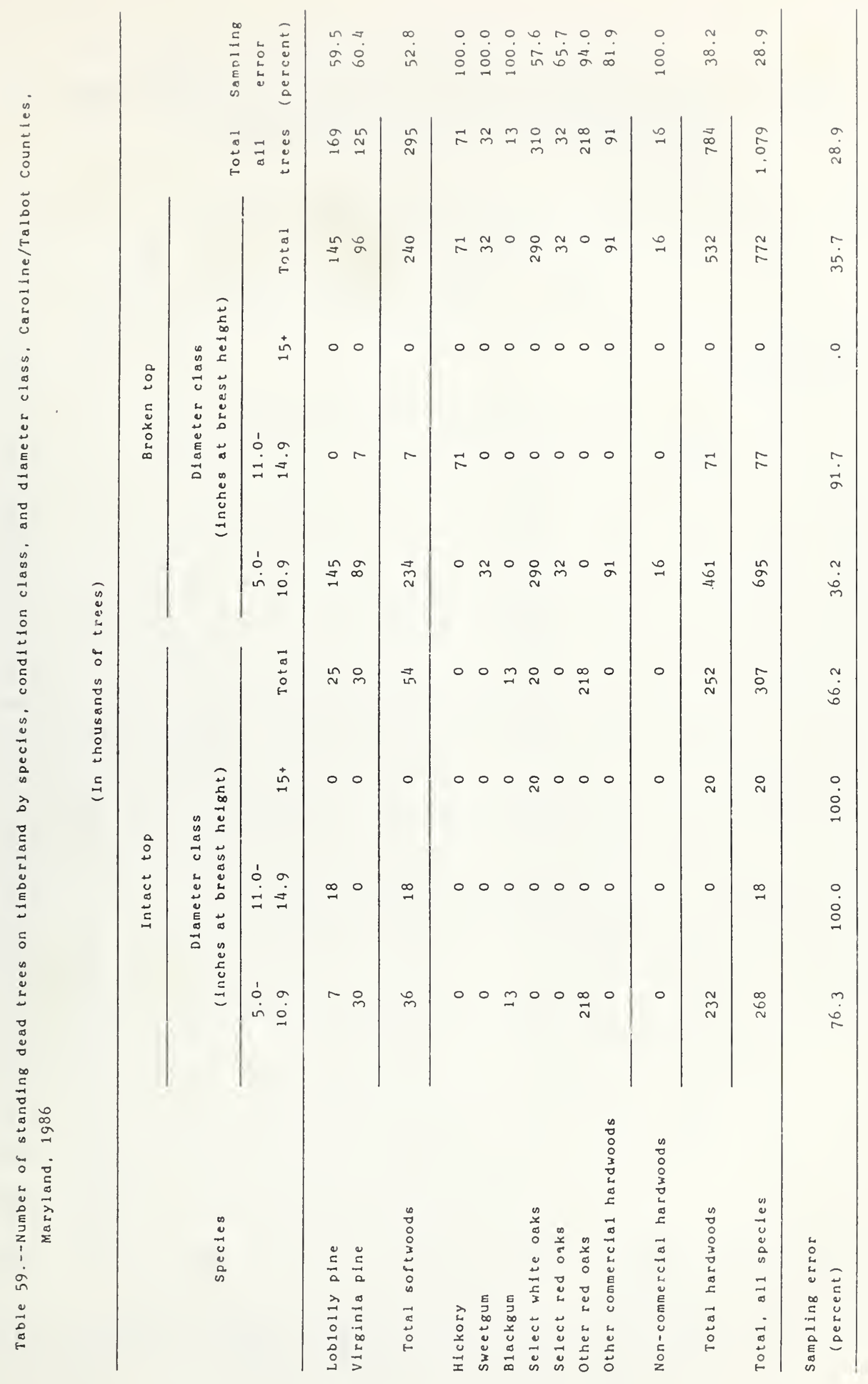


Table 60.--Number of seedings, saplings, and shrubs on timberland by species and browse-utilization class, Caroline/Talbot counties.

Maryland, 1986

(In millions of stems)

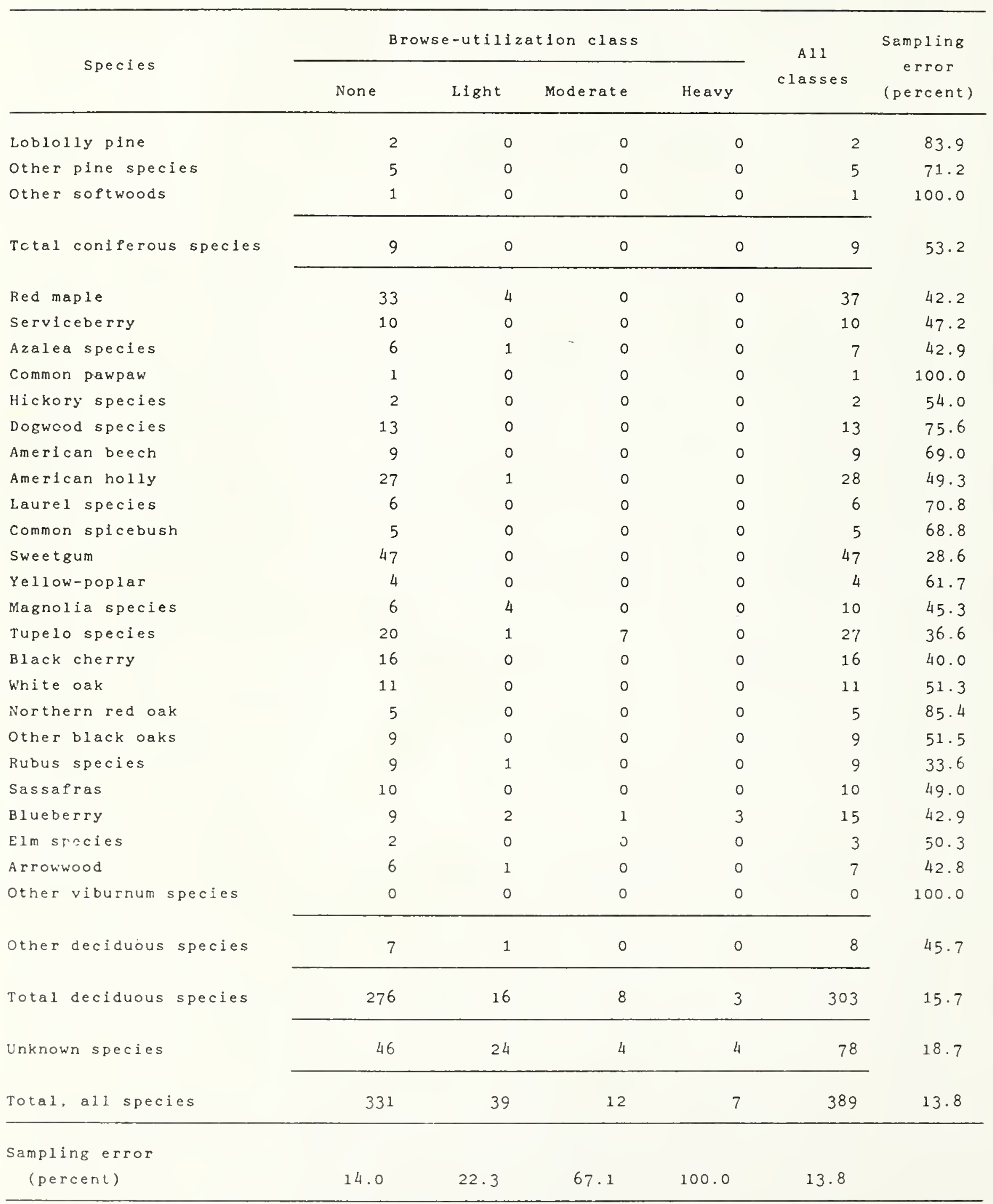









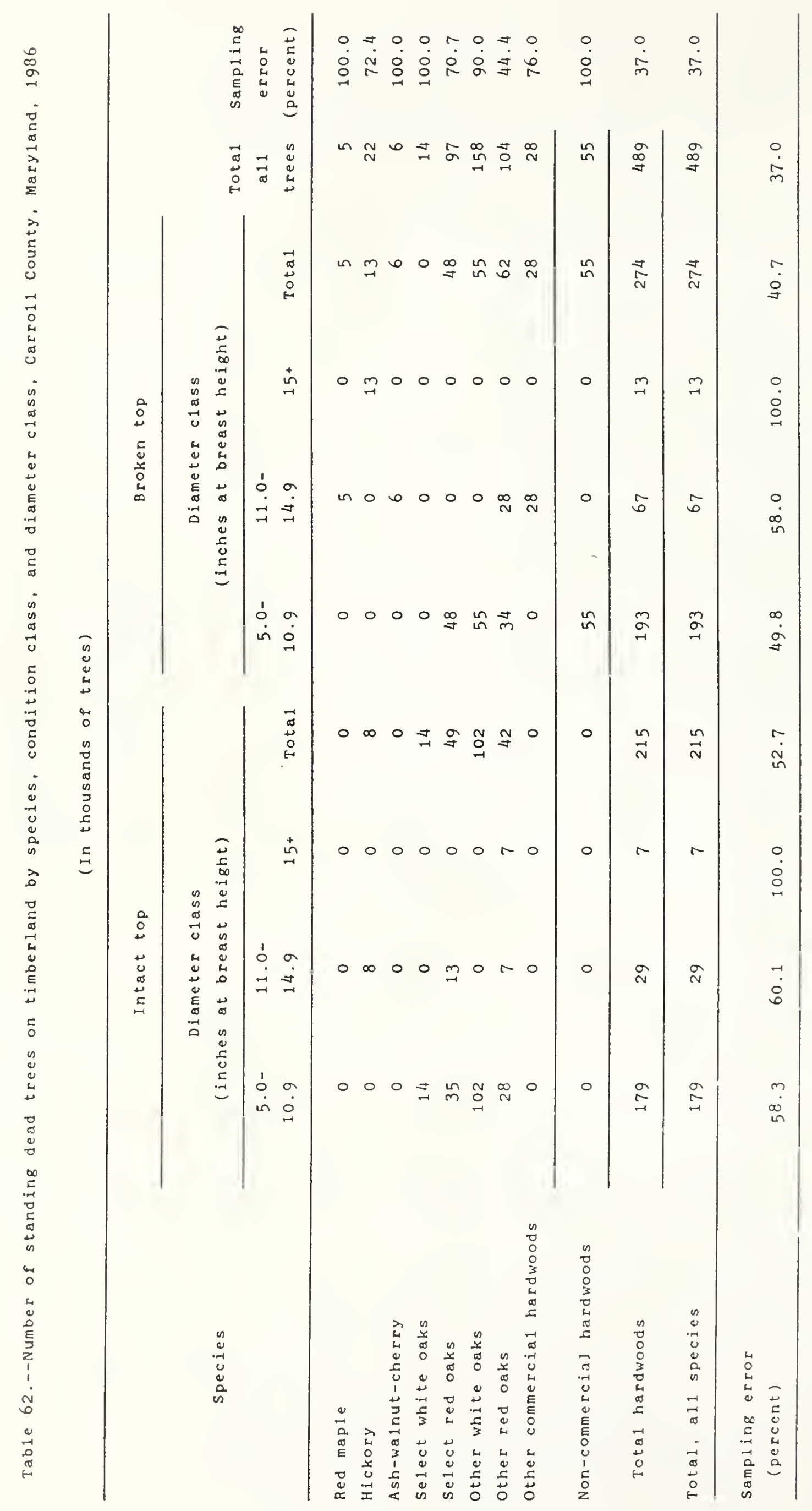


Table 63.--Number of seedings, saplings, and shrubs on timberland by species and browse-utilization class, Carroll County, Maryland, 1986

(In millions of stems)

\begin{tabular}{|c|c|c|c|c|c|c|}
\hline \multirow{2}{*}{ Species } & \multicolumn{4}{|c|}{ Browse-utilization class } & \multirow{2}{*}{$\begin{array}{c}\text { A } 11 \\
\text { classes }\end{array}$} & \multirow{2}{*}{$\begin{array}{c}\text { Sampling } \\
\text { error } \\
\text { (percent) }\end{array}$} \\
\hline & None & Light & Moderate & Heavy & & \\
\hline Red maple & 40 & 0 & 0 & 0 & 40 & $50 \cdot 5$ \\
\hline Serviceberry & 3 & 0 & 0 & 0 & 3 & 79.6 \\
\hline Azalea species & 3 & 0 & 0 & 0 & 3 & 100.0 \\
\hline Common pawpew & 1 & 0 & 0 & 0 & 1 & 100.0 \\
\hline Hickory spectes & 13 & 1 & 1 & 0 & 16 & 35.0 \\
\hline Dogwood spectes & 25 & 0 & 2 & 0 & 27 & $41 \cdot 7$ \\
\hline American beech & 2 & 0 & 0 & 0 & 2 & 100.0 \\
\hline Ash species & 4 & 0 & 0 & 0 & 4 & 58.2 \\
\hline WItch-hazel & 1 & 0 & 0 & 0 & 1 & 100.0 \\
\hline Laurel species & 3 & 0 & 0 & 0 & 3 & $74 \cdot 3$ \\
\hline Common spicebush & 14 & 0 & 0 & 0 & 14 & 43.6 \\
\hline Tupelo species & 16 & 0 & 1 & 0 & 18 & 41.1 \\
\hline Black cherry & 110 & 0 & 1 & 0 & 111 & $57 \cdot 4$ \\
\hline Other cherry species & 1 & 0 & 0 & 0 & 1 & 100.0 \\
\hline White oak & 9 & 1 & 0 & 0 & 10 & $50 \cdot 7$ \\
\hline Chestnut oak & 17 & 0 & 0 & 0 & 17 & $46 \cdot 3$ \\
\hline Northern red oak & 10 & 0 & 0 & 0 & 10 & 93.4 \\
\hline Other black oaks & 12 & 0 & 0 & 0 & 12 & $51 \cdot 9$ \\
\hline Rose species & 15 & 0 & 0 & 0 & 15 & 40.0 \\
\hline Rubus species & 30 & 0 & 0 & 0 & 30 & 36.2 \\
\hline Sassafras & 2 & 0 & 0 & 0 & 2 & $73 \cdot 2$ \\
\hline Blueberry & 10 & 2 & 0 & 0 & 12 & $41 \cdot 7$ \\
\hline Maple-1eaved viburnum & 2 & 0 & 0 & 0 & 2 & 100.0 \\
\hline Arrownood & 7 & 0 & 0 & 0 & 7 & 49.8 \\
\hline Other viburnum species & 4 & 0 & 1 & 0 & 5 & $63 \cdot 5$ \\
\hline Other deciduous species & 23 & 0 & 0 & 0 & 23 & $37 \cdot 6$ \\
\hline Total deciduous species & 376 & 4 & 6 & 0 & 386 & 24.4 \\
\hline Unknown species & 0 & 0 & 0 & 0 & 0 & 100.0 \\
\hline Tota1, all species & 376 & 4 & 6 & 0 & 386 & $24 \cdot 3$ \\
\hline Sampling error & & & & & & \\
\hline$(\operatorname{percen} t)$ & 25.2 & 46.6 & 70.8 & 0 & $24 \cdot 3$ & \\
\hline
\end{tabular}









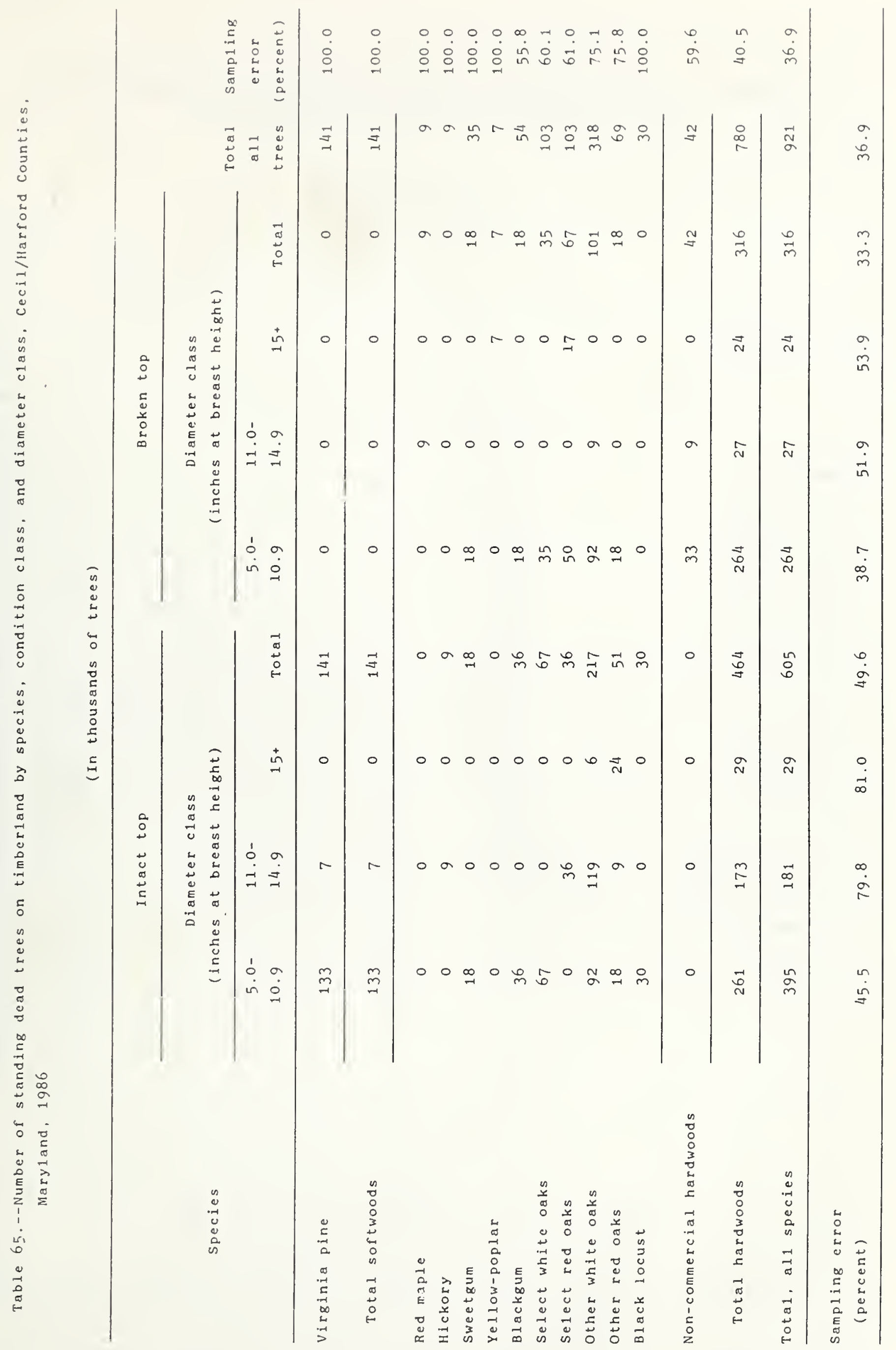


Table 66.--Number of seediings, saplings, and shrubs on timberland by species and browse-utilization class. Cecil/Harford counties.

Maryland, 1986

(In millions of stems)

\begin{tabular}{|c|c|c|c|c|c|c|}
\hline \multirow{2}{*}{ Species } & \multicolumn{4}{|c|}{ Browse-utilization class } & \multirow{2}{*}{$\begin{array}{c}\text { A11 } \\
\text { classes }\end{array}$} & \multirow{2}{*}{$\begin{array}{c}\text { Sampling } \\
\text { error } \\
\text { (percent) }\end{array}$} \\
\hline & None & Light & Moderate & Heavy & & \\
\hline Other pine species & 5 & 0 & 0 & 0 & 5 & 71.8 \\
\hline Other softwoods & 2 & 0 & 0 & 0 & 2 & 70.7 \\
\hline Total coniferous species & 7 & 0 & 0 & 0 & 7 & 56.4 \\
\hline Red maple & 99 & 4 & 0 & 0 & 103 & 35.8 \\
\hline Sugar maple & 1 & 0 & 0 & 0 & 1 & 100.0 \\
\hline Azalea species & 8 & 3 & 0 & 0 & 11 & 33.4 \\
\hline Common pawpaw & 41 & 15 & 0 & 0 & 56 & $69 \cdot 3$ \\
\hline Birch species & 37 & 0 & 0 & 0 & 37 & 74.9 \\
\hline Hickory species & 16 & 2 & 0 & 0 & 18 & 32.6 \\
\hline Dogwood specles & 43 & 3 & 0 & 0 & 46 & 44.9 \\
\hline American beech & 43 & 3 & 2 & 0 & 48 & $45 \cdot 3$ \\
\hline Ash species & 27 & 6 & 2 & 0 & 35 & $32 \cdot 1$ \\
\hline Laurel species & 9 & 1 & 0 & 0 & 10 & 38.6 \\
\hline Common spicebush & 22 & 3 & 1 & 0 & 26 & 28.6 \\
\hline Sweet gum & 35 & 1 & 0 & 0 & 35 & $45 \cdot 3$ \\
\hline Ye110w-poplar & 41 & 0 & 2 & 0 & 43 & 45.0 \\
\hline Tupelo species & 85 & 11 & 0 & 1 & 96 & $34 \cdot 7$ \\
\hline Black cherry & 53 & 25 & 0 & 0 & 78 & $25 \cdot 1$ \\
\hline White oak & 12 & 6 & 0 & 0 & 18 & 49.6 \\
\hline Chestnut oak & 26 & 0 & 4 & 1 & 31 & $71 \cdot 3$ \\
\hline Other white oaks & 4 & 0 & 0 & 0 & 4 & $68 \cdot 5$ \\
\hline Northern red oak & 9 & 0 & 2 & 0 & 12 & $31 \cdot 7$ \\
\hline Other black oaks & 27 & 2 & 0 & 0 & 29 & 59.9 \\
\hline Rose species & 17 & 0 & 1 & 0 & 18 & $38 .=$ \\
\hline Rubus species & 51 & 5 & 1 & 0 & 57 & $21 \cdot 9$ \\
\hline Sassafras & 51 & 6 & 0 & 0 & 57 & 28.8 \\
\hline Blueberry & 14 & 6 & 0 & 0 & 20 & 36.6 \\
\hline Eim species & 6 & 0 & 0 & 0 & 6 & 82.2 \\
\hline Maple-leaved viburnum & 2 & 6 & 0 & 0 & 8 & 56.9 \\
\hline Arrowwood & 13 & 1 & 0 & 0 & 14 & $47 \cdot 3$ \\
\hline Other viburnum species & 25 & 5 & 1 & 0 & 31 & 24.0 \\
\hline Other deciduous species & 28 & 6 & 0 & 0 & 33 & 30.5 \\
\hline Total deciduous species & 847 & 119 & 16 & 2 & 983 & $14 \cdot 7$ \\
\hline Unknown species & 8 & 0 & 0 & 0 & 8 & 49.0 \\
\hline Total, all species & 862 & 119 & 16 & 2 & 998 & $14 \cdot 5$ \\
\hline Sampling error & & & & & & \\
\hline (percent) & 16.0 & $27 \cdot 0$ & $36 \cdot 5$ & $.70 \cdot 7$ & $14 \cdot 5$ & \\
\hline
\end{tabular}







Table 66.--Number of seedlings, saplings, and shrubs on timberland by species and browse-utilization class, Cecil/Harford counties,

Maryland, 1986

(In millions of stems)

\begin{tabular}{|c|c|c|c|c|c|c|}
\hline Species & \multicolumn{4}{|c|}{ Browse-utillzation class } & $\begin{array}{c}\text { A11 } \\
\text { classes }\end{array}$ & $\begin{array}{c}\text { Sampling } \\
\text { error } \\
\text { (percent) }\end{array}$ \\
\hline Other pine species & 5 & 0 & 0 & 0 & 5 & 71.8 \\
\hline Other softwoods & 2 & 0 & 0 & 0 & 2 & 70.7 \\
\hline Total coniferous species & 7 & 0 & 0 & 0 & 7 & 56.4 \\
\hline Red maple & 99 & 4 & 0 & 0 & 103 & 35.8 \\
\hline Sugar maple & 1 & 0 & 0 & 0 & 1 & 100.0 \\
\hline Azalea species & 8 & 3 & 0 & 0 & 11 & $33 \cdot 4$ \\
\hline Birch species & 37 & 0 & 0 & 0 & 37 & 74.9 \\
\hline Hickory species & 16 & 2 & 0 & 0 & 18 & 32.6 \\
\hline Dogwood species & 43 & 3 & 0 & 0 & 46 & 44.9 \\
\hline American beech & 43 & 3 & 2 & 0 & 48 & $45 \cdot 3$ \\
\hline Ash species & 27 & 6 & 2 & 0 & 35 & 32.1 \\
\hline Laurel species & 9 & 1 & 0 & 0 & 10 & 38.6 \\
\hline Common spicebush & 22 & 3 & 1 & 0 & 26 & 28.6 \\
\hline Sweetgum & 35 & 1 & 0 & 0 & 35 & 45.3 \\
\hline Yellow-poplar & 41 & 0 & 2 & 0 & 43 & 45.0 \\
\hline Tupelo species & 85 & 11 & 0 & 1 & 96 & 34.7 \\
\hline Black cherry & 53 & 25 & 0 & 0 & 78 & $25 \cdot 1$ \\
\hline other white oaks & 4 & 0 & 0 & 0 & 4 & $68 \cdot 5$ \\
\hline Northern red oak & 9 & 0 & 2 & 0 & 12 & $31 \cdot 7$ \\
\hline Other black oaks & 27 & 2 & 0 & 0 & 29 & $59 \cdot 9$ \\
\hline Rose species & 17 & 0 & 1 & 0 & 18 & $38 \cdot \because$ \\
\hline Rubus species & 51 & 5 & 1 & 0 & 57 & $21 \cdot 9$ \\
\hline Sassafras & 51 & 6 & 0 & 0 & 57 & 28.8 \\
\hline Blueberry & 14 & 6 & 0 & 0 & 20 & 36.6 \\
\hline Elm species & 6 & 0 & 0 & 0 & 5 & 82.2 \\
\hline Maple-leaved viburnum & 2 & 6 & 0 & 0 & 8 & 56.9 \\
\hline Arrowwood & 13 & 1 & 0 & 0 & 14 & $47 \cdot 3$ \\
\hline Other viburnum species & 25 & 5 & 1 & 0 & 31 & 24.0 \\
\hline Other deciduous species & 28 & 6 & 0 & 0 & 33 & $30 \cdot 5$ \\
\hline Total deciduous species & 847 & 119 & 16 & 2 & 983 & $14 \cdot 7$ \\
\hline Unknown species & 8 & 0 & 0 & 0 & 8 & 49.0 \\
\hline Total, all species & 862 & 119 & 16 & 2 & 998 & $14 \cdot 5$ \\
\hline Sampling error & & & & & & \\
\hline (percent) & 16.0 & $27 \cdot 0$ & $36 \cdot 5$ & .70 .7 & $14 \cdot 5$ & \\
\hline
\end{tabular}












Table 69.--Number of seedlings. saplings, and shrubs on timberland ty species and browse-utilization class. Charles County, Maryland. 1986

( In millions of stems)

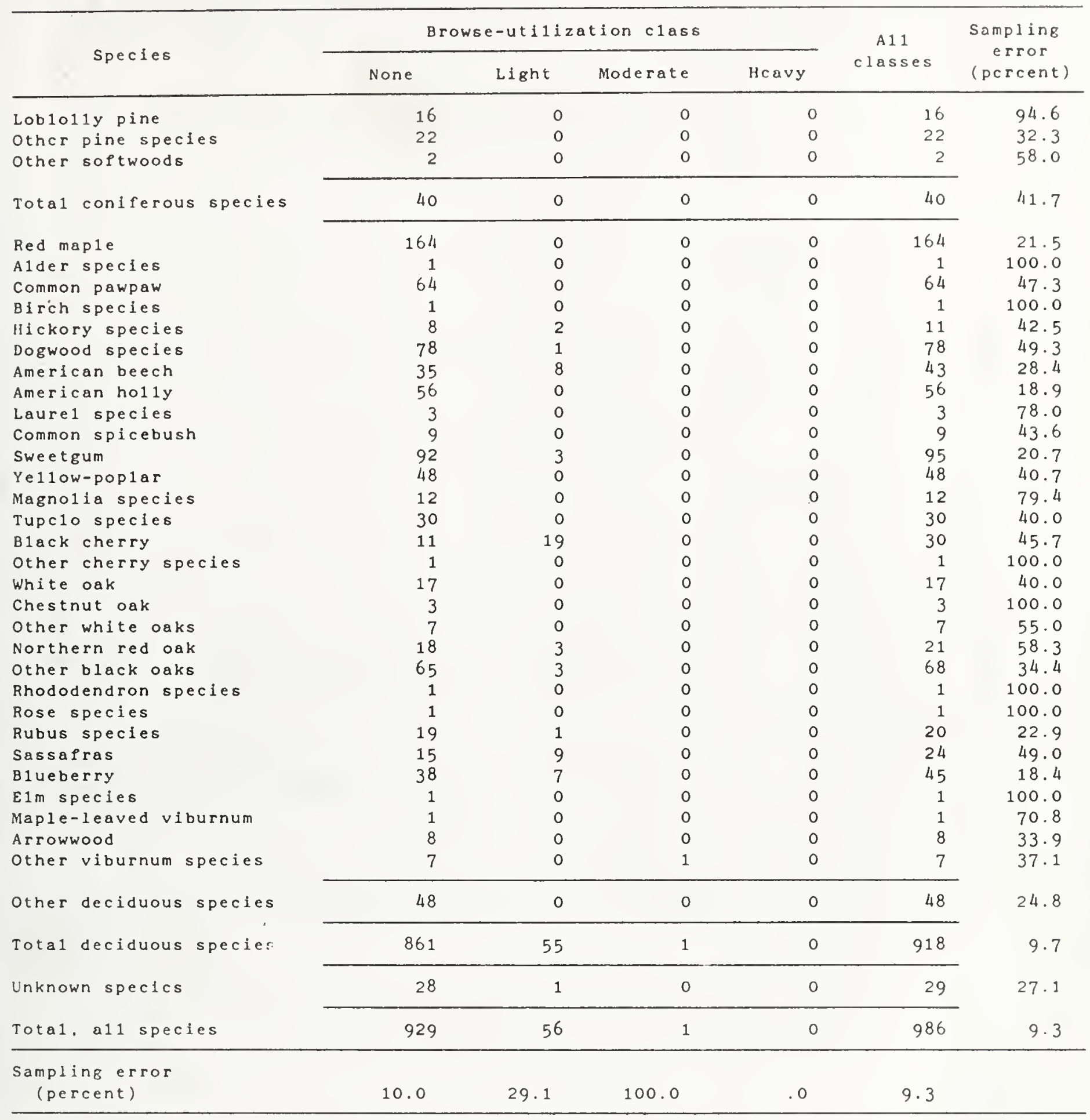












Tab1e 72.- Number of seedlings, saplings, and shrubs on timberland by species and browse-utizization class, Dorchester County, Maryland, 1986

(In militons of stems)

\begin{tabular}{|c|c|c|c|c|c|c|}
\hline \multirow{2}{*}{ Species } & \multicolumn{4}{|c|}{ Browse-utilization class } & \multirow{2}{*}{$\begin{array}{c}\text { A11 } \\
\text { c1asses }\end{array}$} & \multirow{2}{*}{$\begin{array}{l}\text { Sampling } \\
\text { error } \\
\text { (percent) }\end{array}$} \\
\hline & None & Light & Moderate & Heavy & & \\
\hline Loololly pine & 52 & 0 & 0 & 1 & 53 & 41.8 \\
\hline Other pine species & 2 & 0 & 0 & 0 & 2 & 100.0 \\
\hline Other softwoods & 1 & 0 & 0 & 0 & 1 & 70.9 \\
\hline Total coniferous species & 55 & 0 & 0 & 1 & 56 & 39.6 \\
\hline Red maple & 87 & 6 & 1 & 1 & 96 & 33.8 \\
\hline Alder species & 4 & 2 & 0 & 0 & 6 & 71.5 \\
\hline Serviceberry & 1 & 0 & 0 & 0 & 1 & 100.0 \\
\hline Azālea species & 1 & 0 & 0 & 0 & 1 & 100.0 \\
\hline Dogwood species & 10 & 0 & 0 & 0 & 10 & 93.8 \\
\hline American beech & 2 & 0 & 0 & 0 & 2 & 100.0 \\
\hline American holly & 26 & 0 & 5 & 1 & 32 & 29.0 \\
\hline Laure1 species & 1 & 0 & 0 & 0 & 1 & 100.0 \\
\hline Sweetgum & 36 & 4 & 0 & 4 & 43 & 31.7 \\
\hline Magnolia species & 8 & 2 & 0 & 0 & 10 & 39.0 \\
\hline Tupe1o species & 50 & 4 & 0 & 4 & 57 & 39.6 \\
\hline B1ack cherry & 8 & 0 & 0 & 0 & 8 & 57.2 \\
\hline Other cherry species & 0 & 0 & 0 & 2 & 2 & 100.0 \\
\hline White oak & 9 & 0 & 0 & 0 & 9 & 77.6 \\
\hline other white oaks & 7 & 0 & 0 & 0 & 7 & 61.9 \\
\hline other black oaks & 10 & 2 & 0 & 0 & 12 & 35.0 \\
\hline Rose species & 1 & 1 & 0 & 0 & 1 & 70.9 \\
\hline Rubus species & 7 & 0 & 0 & 0 & 7 & 38.0 \\
\hline Sassafras & 6 & 0 & 0 & 0 & 6 & 53.4 \\
\hline Blueberry & 30 & 11 & 5 & 1 & 47 & 16.7 \\
\hline Arrowwood & 1 & 0 & 0 & 0 & 1 & 100.0 \\
\hline Other viburnum species & 1 & 0 & 0 & 0 & 1 & 100.0 \\
\hline Other deciduous species & 11 & 0 & 1 & 1 & 13 & 33.1 \\
\hline Total deciduous species & 319 & 31 & 11 & 14 & 376 & $13 \cdot 7$ \\
\hline Uraknown species & 43 & 7 & 2 & 1 & 54 & 18.9 \\
\hline Tota1, a11 species & 418 & 38 & 14 & 16 & 486 & 11.6 \\
\hline Sampling error & & & & & & \\
\hline$(\operatorname{percen} t)$ & 12.8 & 28.1 & 38.0 & 46.4 & 11.6 & \\
\hline
\end{tabular}




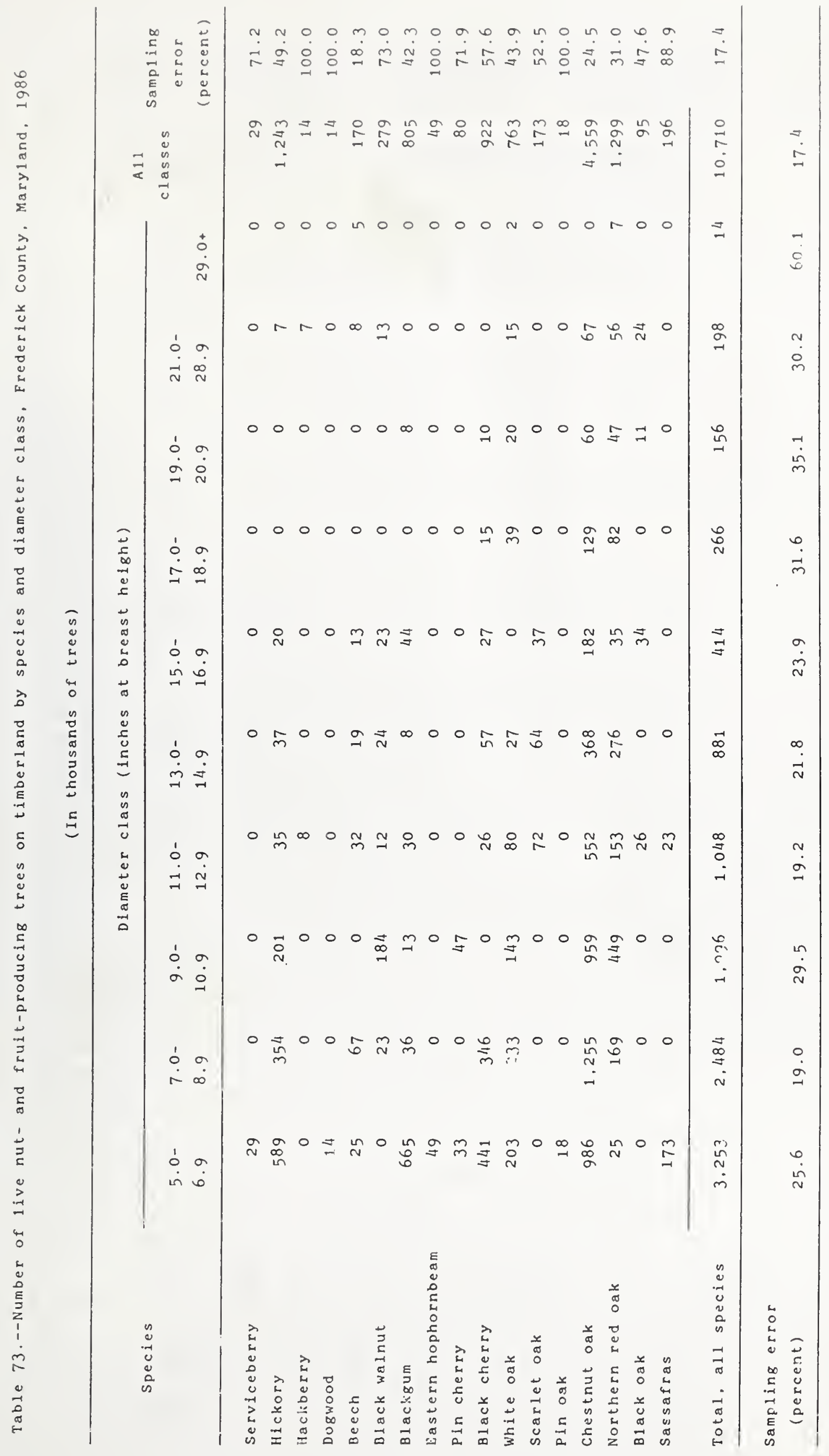







Tab1e 75.--Number of seedlings, saplings, and shrubs on timberland by species and browse-ut 111 zation class, Frederick County, Maryland, 1986

(In m111ions of stems)

\begin{tabular}{|c|c|c|c|c|c|c|}
\hline \multirow{2}{*}{ Species } & \multicolumn{4}{|c|}{ Browse-ut1lization class } & \multirow{2}{*}{$\begin{array}{c}\text { All } \\
\text { classes }\end{array}$} & \multirow{2}{*}{$\begin{array}{c}\text { Sampling } \\
\text { error } \\
\text { (percent) }\end{array}$} \\
\hline & None & $\operatorname{Light}$ & Moderate & Heavy & & \\
\hline Other pine species & 3 & 0 & 0 & 0 & 3 & 100.0 \\
\hline other softwoods & 2 & 0 & 0 & 0 & 2 & 100.0 \\
\hline Total confferous species & 4 & 0 & 0 & 0 & 4 & $72 \cdot 3$ \\
\hline Red maple & 41 & 21 & 0 & 0 & 63 & $35 \cdot 5$ \\
\hline Other maple species & 17 & 0 & 0 & 0 & 17 & $69 \cdot 5$ \\
\hline A1der species & 1 & 0 & 0 & 0 & 1 & 100.0 \\
\hline Serviceberry & 9 & 0 & 0 & 0 & 9 & 63.4 \\
\hline Azalea species & 6 & 0 & 0 & 0 & 6 & 59.0 \\
\hline BIrch specles & 2 & 0 & 0 & 0 & 2 & $75 \cdot 9$ \\
\hline Hickory species & 9 & 1 & 0 & 0 & 9 & $35 \cdot 5$ \\
\hline Dogwood species & 18 & 0 & 0 & 0 & 18 & $55 \cdot 7$ \\
\hline Amerlcan beech & 6 & 0 & 0 & 0 & 6 & $61 \cdot 3$ \\
\hline Ash specles & 34 & 0 & 0 & 0 & 34 & 46.4 \\
\hline Huckleberry & 5 & 0 & 0 & 0 & 5 & $51 \cdot 1$ \\
\hline Witch-hazel & 2 & 0 & 0 & 1 & 3 & $78 \cdot 5$ \\
\hline Laure1 specles & 12 & 0 & 0 & 0 & 12 & 48.6 \\
\hline Common spicebush & 21 & 0 & 0 & 0 & 21 & $37 \cdot 2$ \\
\hline Yellow-poplar & 2 & 0 & 0 & 0 & 2 & 100.0 \\
\hline Tupelo specles & 9 & 2 & 0 & 0 & 11 & $50 \cdot 3$ \\
\hline Black cherry & 25 & 2 & 0 & 0 & 27 & 40.1 \\
\hline Other cherry specles & 6 & 0 & 0 & 0 & 6 & $71 \cdot 4$ \\
\hline White oak & 1 & 0 & 0 & 0 & 1 & 100.0 \\
\hline Chestnut oak & 41 & 5 & 0 & 0 & 45 & 50.8 \\
\hline Northern red oak & 4 & 2 & 0 & 0 & 6 & 49.1 \\
\hline Other b1ack oaks & 1 & 0 & 0 & 0 & 1 & 100.0 \\
\hline Rose species & 10 & 1 & 0 & 0 & 11 & $72 \cdot 7$ \\
\hline Rubus species & 29 & 0 & 0 & 0 & 29 & 28.1 \\
\hline Sassafras & 22 & 3 & 0 & 0 & 25 & $52 \cdot 1$ \\
\hline Blueberry & 17 & 1 & 0 & 0 & 18 & 28.1 \\
\hline Elm species & 21 & 5 & o & 0 & 26 & 59.0 \\
\hline Arrowhood & 1 & 0 & 0 & 0 & 1 & 100.0 \\
\hline Other viburnum species & 1 & 0 & 0 & 0 & 1 & $71 \cdot 7$ \\
\hline Other deciduous species & 16 & 1 & 0 & 0 & 17 & 48.0 \\
\hline Total deciduous species & 390 & 44 & 0 & 1 & 435 & 14.4 \\
\hline Unknown species & 1 & 0 & 0 & 0 & 1 & 100.0 \\
\hline Tota1, a11 species & 395 & 44 & 0 & 1 & 440 & 14.4 \\
\hline Sampling error & & & • & & & \\
\hline (percent) & $15 \cdot 5$ & 70.9 & .0 & 100.0 & 14.4 & \\
\hline
\end{tabular}




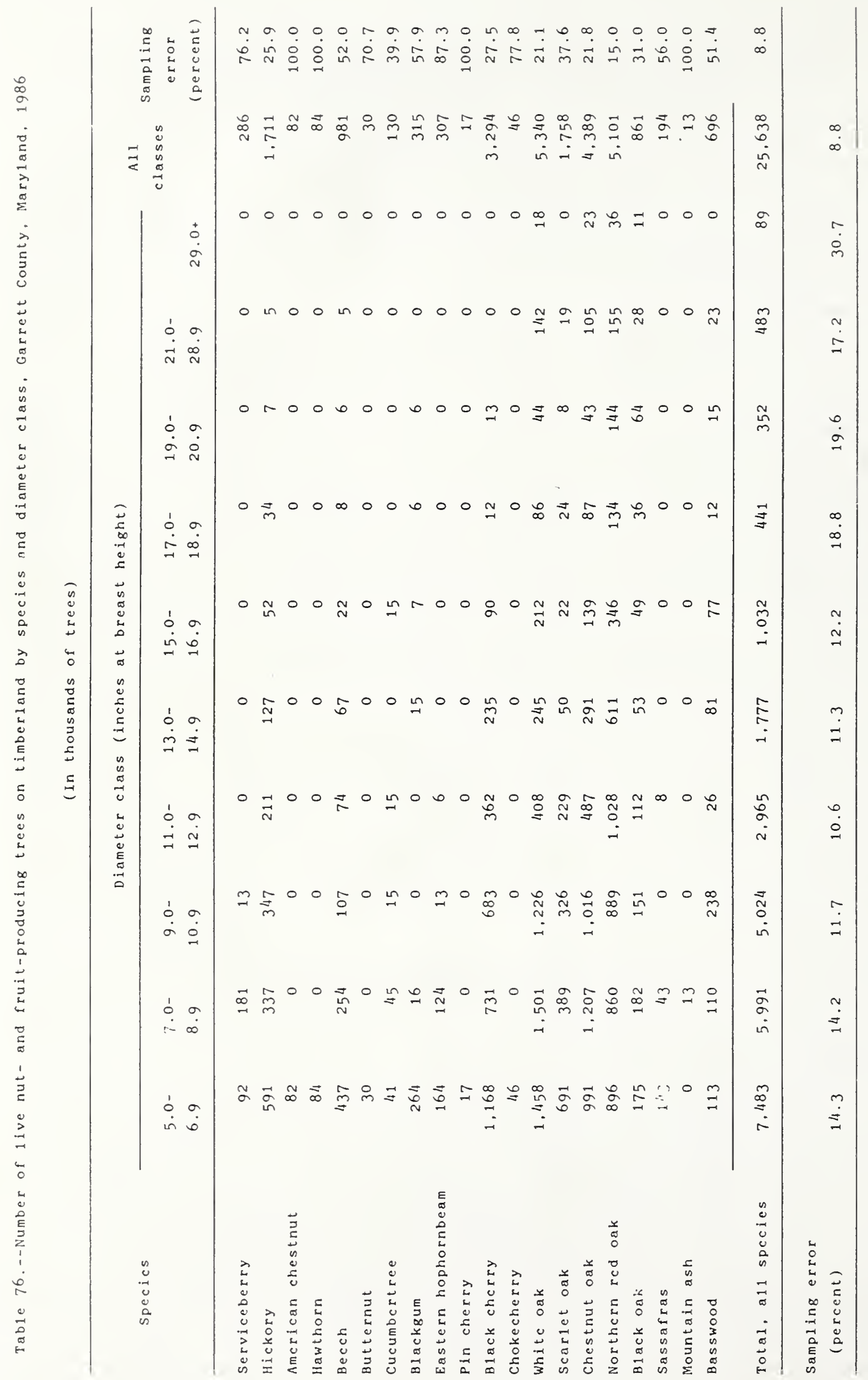




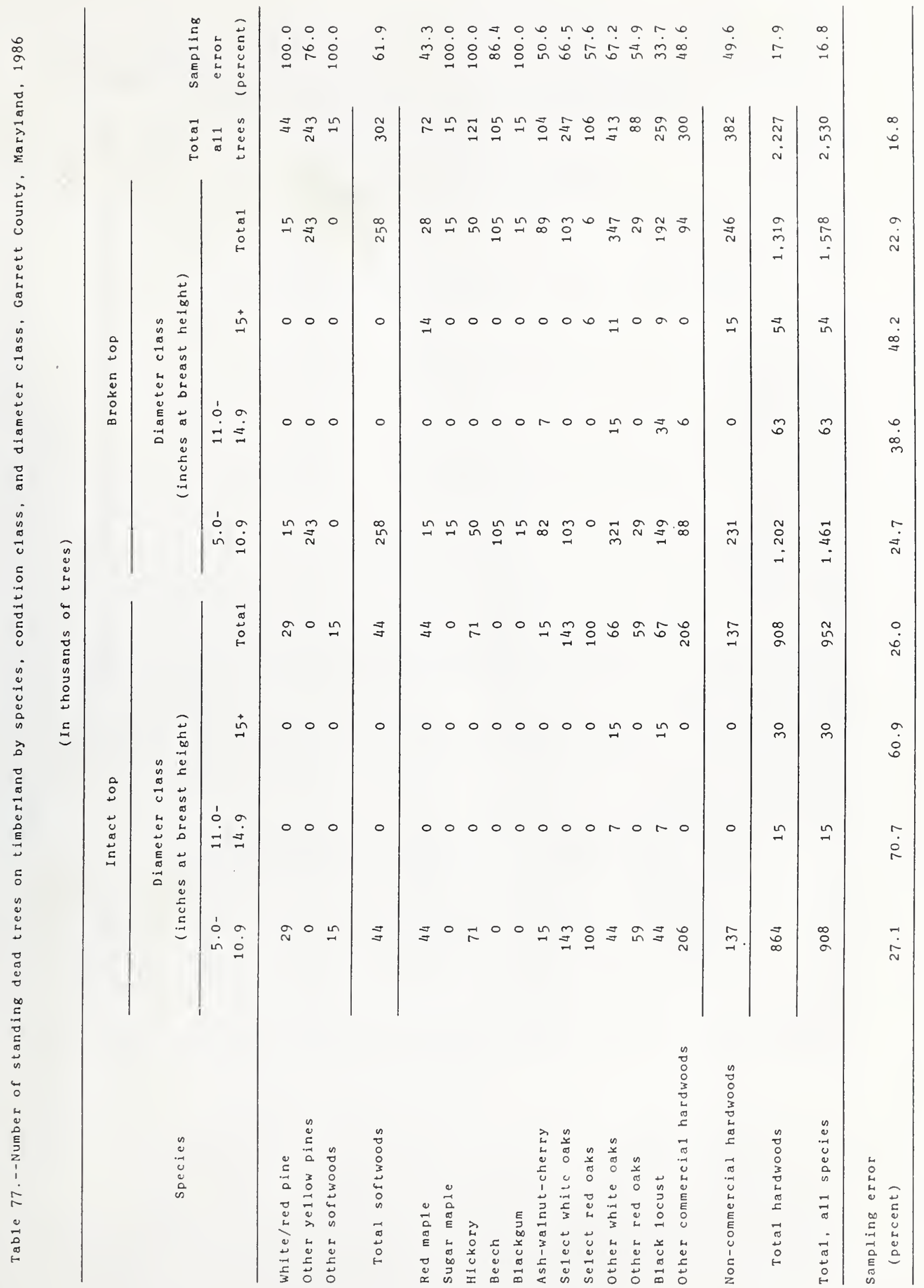


Tab1e 78.--Number of seedings, sap11ngs, and shrubs on timberland by species and browse-utilization class, Garrett County, Maryland, 1986

(In mi11ions of stems)

\begin{tabular}{|c|c|c|c|c|c|c|}
\hline \multirow{2}{*}{ Species } & \multicolumn{4}{|c|}{ Browse-uti11zation c1ass } & \multirow{2}{*}{$\begin{array}{c}\text { Al1 } \\
\text { classes }\end{array}$} & \multirow{2}{*}{$\begin{array}{c}\text { Sampling } \\
\text { error } \\
\text { (percent) }\end{array}$} \\
\hline & None & Light & Moderate & Heavy & & \\
\hline Other pine specles & 6 & 0 & 0 & 0 & 6 & 45.9 \\
\hline Other softwoods & 14 & 0 & 0 & 0 & 14 & 34.2 \\
\hline Tota1 coniferous specles & 21 & 0 & 0 & 0 & 21 & 26.9 \\
\hline Red mal e & 256 & 59 & 12 & 0 & 327 & 21.2 \\
\hline Sugar muple & 55 & 29 & 6 & 0 & 91 & 32.4 \\
\hline Other map1e species & 89 & 2 & 0 & 2 & 93 & $31 \cdot 3$ \\
\hline Serviceberry & 86 & 15 & 1 & 0 & 102 & 28.6 \\
\hline Azalea species & 12 & 0 & 0 & 0 & 12 & $41 \cdot 5$ \\
\hline Blrch species & 62 & 3 & 4 & 0 & 69 & 26.6 \\
\hline Hickory species & 13 & 1 & 0 & 0 & 14 & 34.0 \\
\hline American beech & 8 & 3 & 0 & 0 & 11 & 34.6 \\
\hline Ash species & 52 & 10 & 2 & 0 & 64 & $46 \cdot 3$ \\
\hline Huck1eberry & 31 & 3 & 0 & 0 & 34 & 27.0 \\
\hline Witch-haze 1 & 33 & 4 & 1 & 0 & 39 & 16.8 \\
\hline Laure 1 species & 18 & 0 & 1 & 0 & 19 & 34.2 \\
\hline Ye11ow-pop1ar & 5 & 0 & 0 & 0 & 5 & 85.8 \\
\hline Magnolia specles & 8 & 0 & 0 & 0 & 8 & 66.2 \\
\hline Tupe1o species & 18 & 0 & 1 & 1 & 21 & 42.2 \\
\hline B1ack cherry & 167 & 63 & 4 & 0 & 233 & $15 \cdot 5$ \\
\hline Other cherry spectes & 6 & 0 & 0 & 0 & 6 & $72 \cdot 3$ \\
\hline White oak & 82 & 3 & 1 & 0 & 86 & 36.2 \\
\hline Chestnut oak & 16 & 1 & 3 & 0 & 20 & $33 \cdot 5$ \\
\hline Northern red oak & 49 & 6 & 4 & 0 & 59 & 28.0 \\
\hline Other b1ack oaks & 10 & 2 & 0 & 0 & 13 & 41.8 \\
\hline Rhododendron species & 17 & 0 & 0 & 0 & 17 & 43.0 \\
\hline B1ueberry & 11 & 1 & 1 & 0 & 12 & $37 \cdot 5$ \\
\hline Elm species & 4 & 0 & 0 & 0 & 4 & 73.1 \\
\hline Map1e-1eaved viburnum & 4 & 2 & 1 & 0 & 7 & 46.9 \\
\hline Arrowwood & 3 & 1 & 1 & 0 & 5 & 54.3 \\
\hline Other viburnum species & 2 & 1 & 0 & 0 & 3 & 61.8 \\
\hline Other decjduous species & 95 & 48 & 0 & 2 & 146 & $23 \cdot 7$ \\
\hline Total deciduous species & 1,421 & 287 & 53 & 6 & 1.766 & 9.2 \\
\hline Unknown species & 22 & 0 & 1 & 0 & 23 & 31.8 \\
\hline Tota1. a11 species & 1.464 & 287 & 53 & 6 & 1.810 & 9.0 \\
\hline Sampling error & & & & & & \\
\hline (percent) & $10 \cdot 3$ & $22 \cdot 3$ & $25 \cdot 2$ & 49.5 & 9.0 & \\
\hline
\end{tabular}




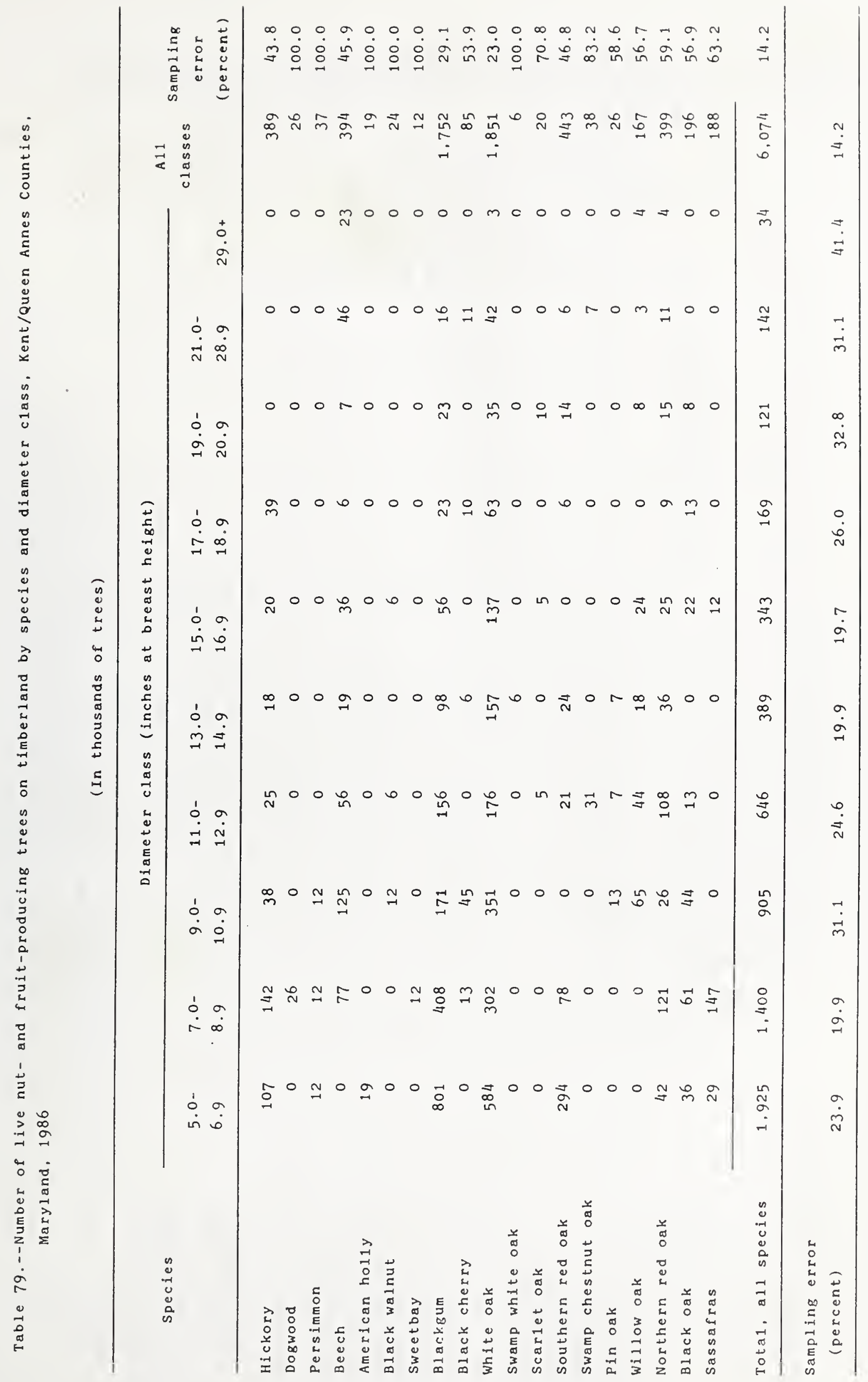




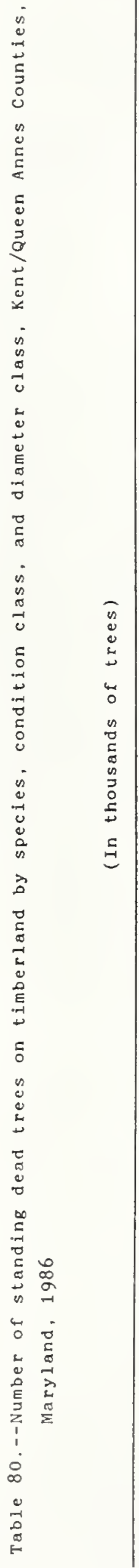

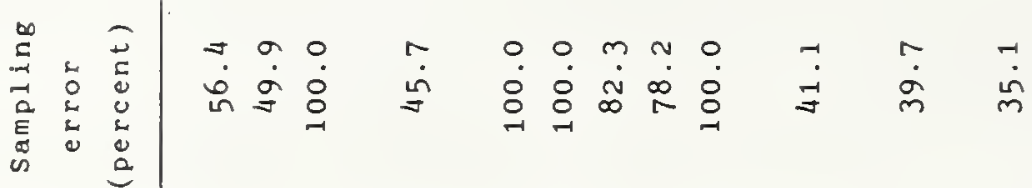

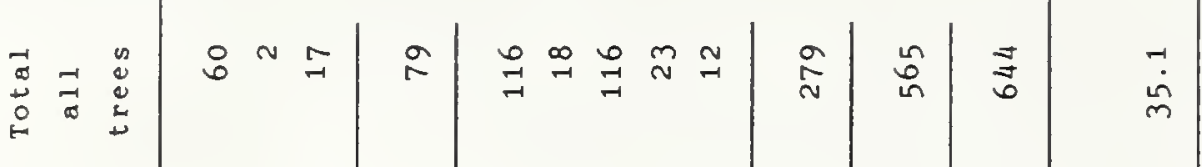



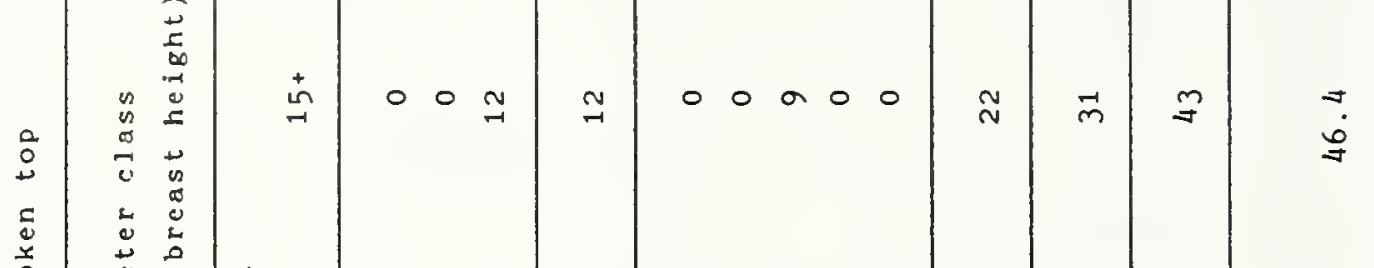

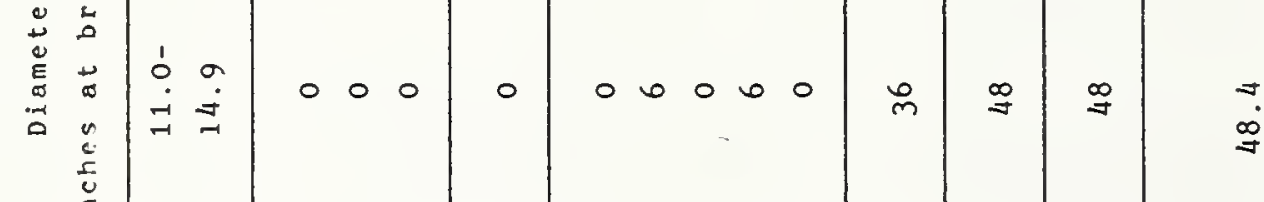

$\Xi$

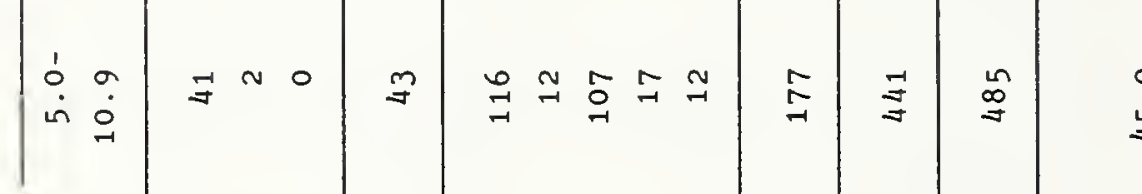




Table 81.--Number of seedlings, saplings, and shrubs on timberland by species

and browse-utilization class. Kent/Queen Annes Counties.

Maryland, 1986

(In militions of stems)

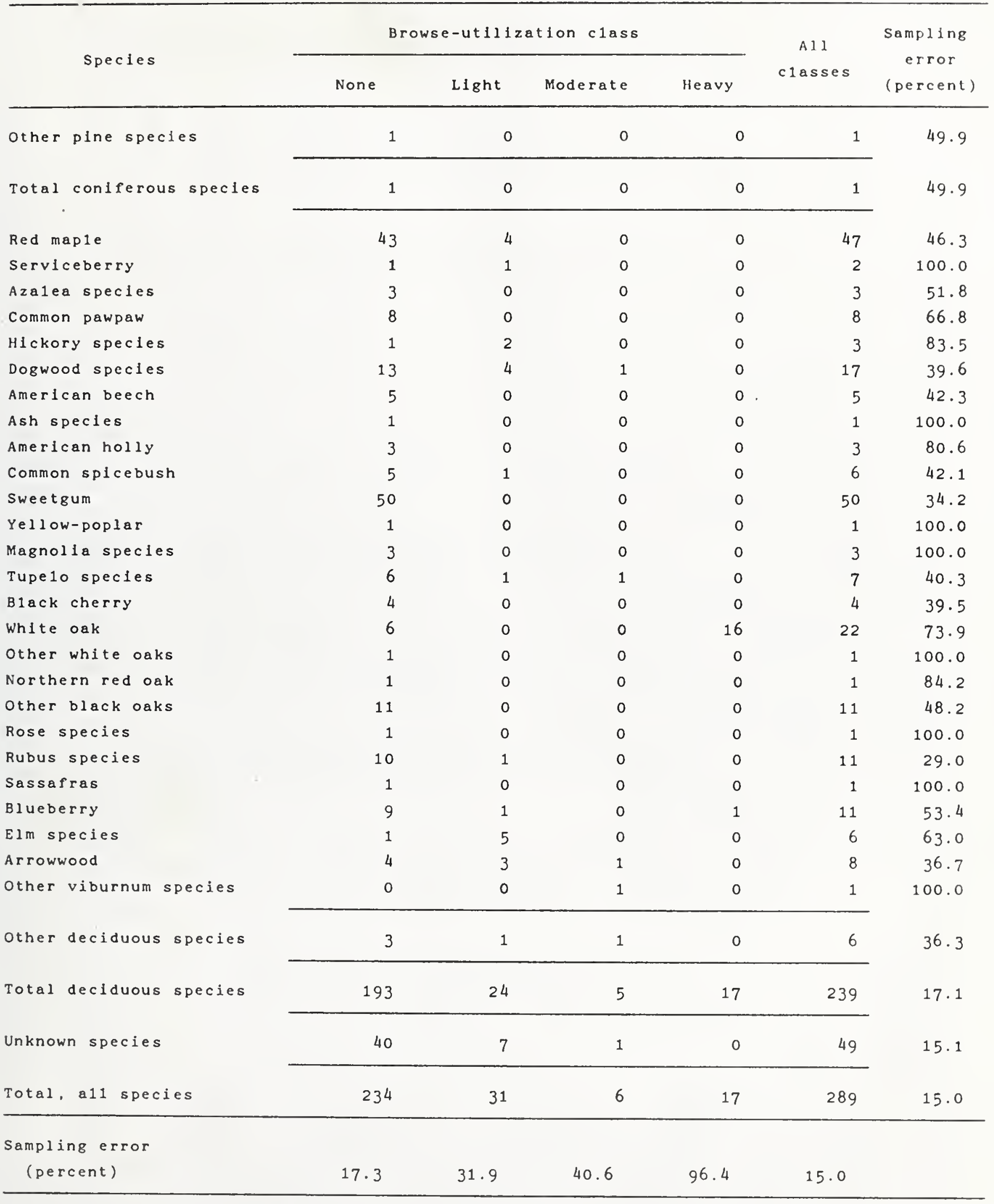




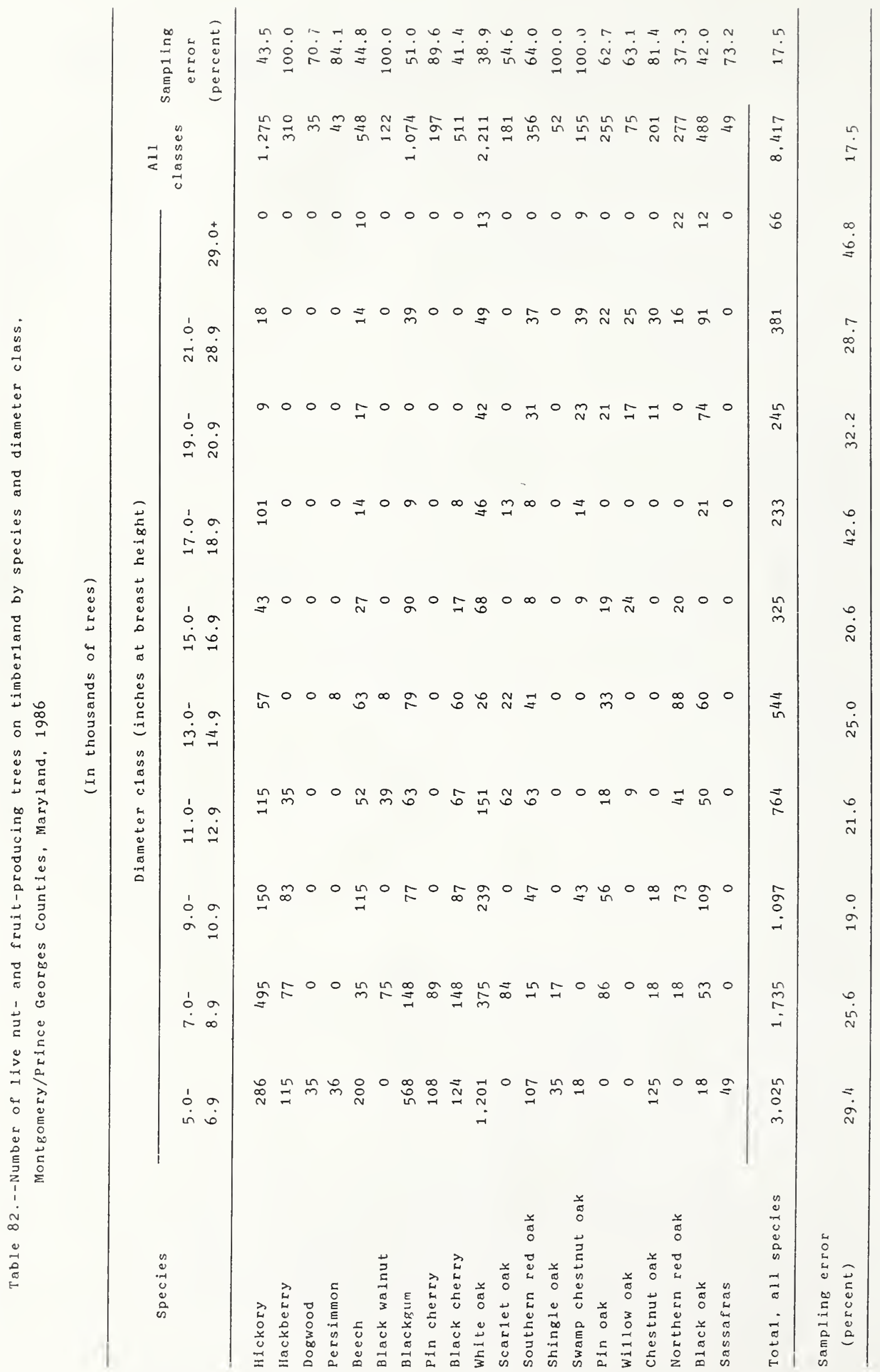




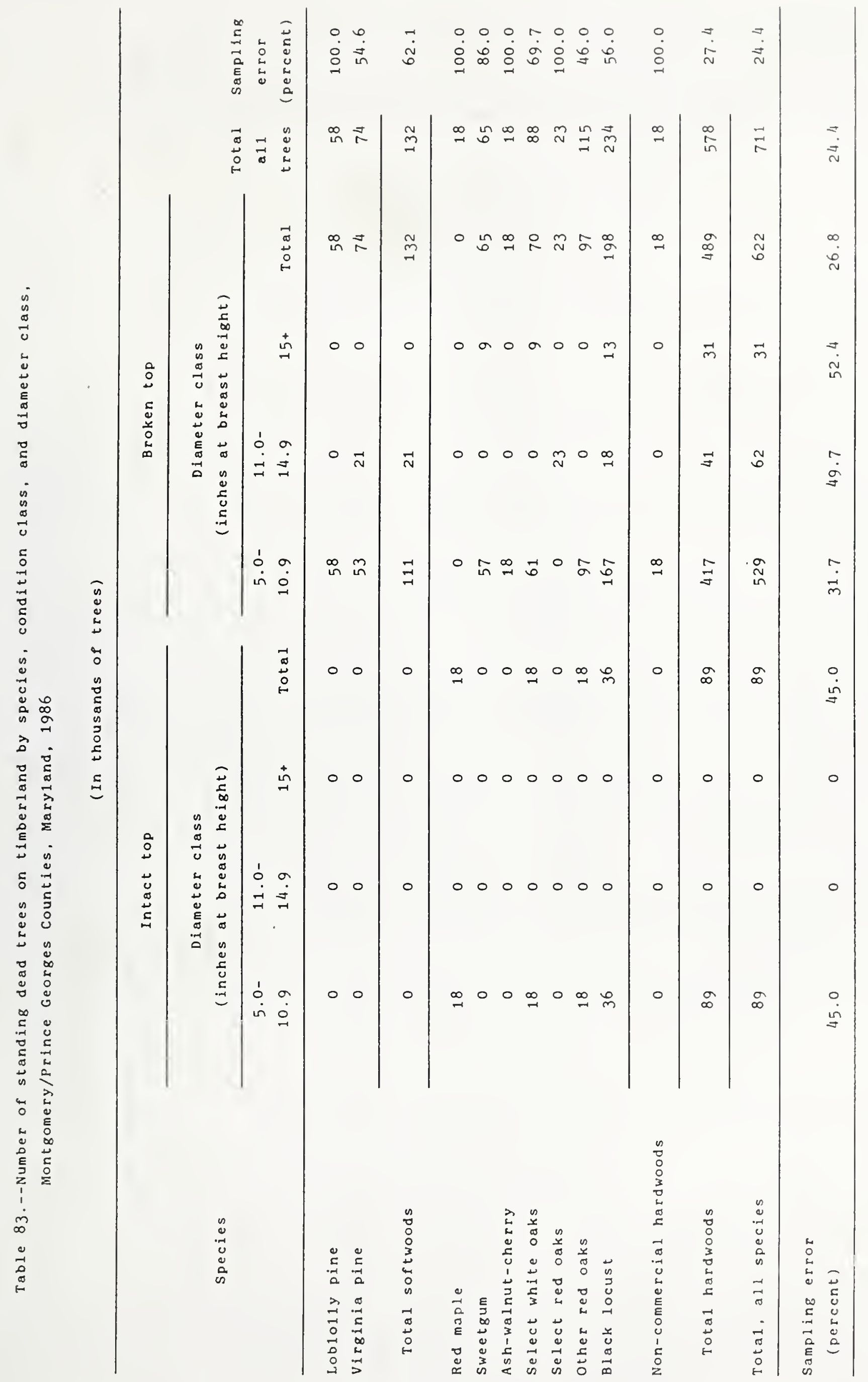


Table 84,--Number of seedlings, saplings, and shrubs on timberland by species and browse-utilization class, Montgomery/Prince Georges Coun:ies. Maryland, 1986

( In millions of stems)

\begin{tabular}{|c|c|c|c|c|c|c|}
\hline \multirow{2}{*}{ Species } & \multicolumn{4}{|c|}{ Browse-utilization class } & \multirow{2}{*}{$\begin{array}{c}\text { Al1 } \\
\text { classes }\end{array}$} & \multirow{2}{*}{$\begin{array}{c}\text { Sampling } \\
\text { error } \\
\text { (percent) }\end{array}$} \\
\hline & None & Light & Moderate & Heavy & & \\
\hline other softwoods & 2 & 0 & 0 & 0 & 2 & 71.1 \\
\hline Total coniferous species & 2 & 0 & 0 & 0 & 2 & 71.1 \\
\hline Red maple & 73 & 0 & 0 & 0 & 73 & 19.3 \\
\hline Alder species & 5 & 0 & 0 & 0 & 5 & $71 \cdot 3$ \\
\hline Common pawpaw & 13 & 0 & 0 & 0 & 13 & 66.9 \\
\hline Birch species & 1 & 0 & 0 & 0 & 1 & 100.0 \\
\hline Hickory species & 13 & 0 & 0 & 0 & 13 & $69 \cdot 5$ \\
\hline Dogwood species & 35 & 0 & 0 & 0 & 35 & $38 \cdot 5$ \\
\hline American beech & 14 & 0 & 0 & 0 & 14 & 43.6 \\
\hline Ash species & 6 & 0 & 0 & 0 & 6 & 100.0 \\
\hline Amcrican holly & 18 & 0 & 0 & 0 & 18 & $51 \cdot 4$ \\
\hline Laurel species & 5 & 0 & 0 & 0 & 5 & 69.2 \\
\hline Common spicebush & 8 & 1 & 0 & 0 & 9 & 49.8 \\
\hline Sweet gum & 54 & 12 & 0 & 0 & 66 & $39 \cdot 7$ \\
\hline Yellow-poplar & 84 & 0 & 0 & 0 & 84 & 60.0 \\
\hline Magnolia species & 1 & 0 & 0 & 0 & 1 & 100.0 \\
\hline Tupelo species & 51 & 3 & 0 & 0 & 54 & $18 \cdot 5$ \\
\hline Black cherry & 18 & 21 & 0 & 0 & 39 & 56.0 \\
\hline Other cherry species & 19 & 0 & 0 & 0 & 19 & 45.8 \\
\hline White oak & 7 & 0 & 0 & 0 & 7 & 52.6 \\
\hline Chestnut oak & 2 & 0 & 0 & 0 & 2 & 100.0 \\
\hline Other white oaks & 13 & 0 & 0 & 0 & 13 & 100.0 \\
\hline Northern red oak & 3 & 0 & 0 & 0 & 3 & $74 \cdot 7$ \\
\hline Other black oaks & 19 & 0 & 0 & 0 & 19 & 38.2 \\
\hline Rose species & 29 & 1 & 0 & 0 & 30 & 56.0 \\
\hline Rubus spocies & 23 & 0 & 0 & 0 & 23 & 28.8 \\
\hline Sassafras & 13 & 0 & 0 & 0 & 13 & $39 \cdot 3$ \\
\hline Blueber:y & 26 & 4 & 0 & 0 & 31 & $27 \cdot 7$ \\
\hline Elm species & 2 & 0 & 0 & 0 & 2 & 100.0 \\
\hline Maple-leaved viburnum & 2 & 0 & 0 & 0 & 2 & 100.0 \\
\hline ArrowwoOd & 11 & 1 & 0 & 0 & 11 & $37 \cdot 0$ \\
\hline other deciduous species & 31 & 0 & 0 & 0 & 31 & $27 \cdot 7$ \\
\hline Total deciduous species & 601 & 44 & 0 & 0 & 645 & 16.8 \\
\hline Unknown species & 13 & 0 & 0 & 0 & 13 & 26.2 \\
\hline Total, all species & 616 & 44 & 0 & 0 & 660 & 16.6 \\
\hline
\end{tabular}

Sampling error

(percent) 










Table 87.--Number of seedlings, saplings, and shrubs on timberland by species and browse-utilization class. St. Marys County, Maryland, 1986

(In millions of stems)

\begin{tabular}{|c|c|c|c|c|c|c|}
\hline \multirow{2}{*}{ Species } & \multicolumn{4}{|c|}{ Browse-utilization class } & \multirow{2}{*}{$\begin{array}{c}\text { AIl } \\
\text { classes }\end{array}$} & \multirow{2}{*}{$\begin{array}{l}\text { Sampling } \\
\text { error } \\
\text { (percent) }\end{array}$} \\
\hline & None & L1 ght & Moderate & Heavy & & \\
\hline Loblo11y pine & 8 & 0 & 0 & 0 & 8 & 52.4 \\
\hline other pine species & 9 & 0 & 0 & 0 & 9 & 69.3 \\
\hline Other softwoods & 1 & 0 & 0 & 0 & 1 & 71.0 \\
\hline Total coniferous species & 18 & 0 & 0 & 0 & 18 & $43 \cdot 3$ \\
\hline Red maple & 53 & 4 & 0 & 0 & 57 & $22 \cdot 3$ \\
\hline Alder species & 3 & 0 & 0 & 0 & 3 & 72.6 \\
\hline Common pawpaw & 10 & 0 & 0 & 0 & 10 & 57.6 \\
\hline Hickory species & 8 & 0 & 0 & 0 & 8 & 40.4 \\
\hline Dogwood species & 20 & 0 & 0 & 0 & 20 & 32.8 \\
\hline American beech & 19 & 0 & 0 & 0 & 19 & 30.1 \\
\hline Ash species & 1 & 0 & 0 & 0 & 1 & 100.0 \\
\hline American holly & 48 & 0 & 0 & 0 & 48 & 20.2 \\
\hline Laurel species & 9 & 0 & 0 & 0 & 9 & $50 \cdot 3$ \\
\hline Common spicebush & 1 & 0 & 0 & 0 & 1 & 71.6 \\
\hline Swe etgum & 43 & 0 & 0 & 0 & 43 & 21.2 \\
\hline Ye1low-poplar & 10 & 0 & 0 & 0 & 10 & $47 \cdot 7$ \\
\hline Magnolia species & 3 & 0 & 0 & 0 & 3 & $81 \cdot 3$ \\
\hline Tupelo species & 21 & 1 & 0 & 0 & 21 & 31.8 \\
\hline B1ack cherry & 3 & 0 & 0 & 0 & 3 & 56.1 \\
\hline Other cherry species & 5 & 0 & 0 & 0 & 5 & 64.7 \\
\hline White oak & 9 & 0 & 0 & 0 & 9 & 28.5 \\
\hline Chestnut oak & 2 & 0 & 0 & 0 & 2 & 77.2 \\
\hline Other white oaks & 3 & 0 & 0 & 0 & 3 & 100.0 \\
\hline Northern red oak & 3 & 0 & 0 & 0 & 3 & $51 \cdot 7$ \\
\hline Other b1ack oaks & 27 & 0 & 0 & 0 & 27 & 32.7 \\
\hline Rose species & 1 & 0 & 0 & 0 & 1 & 100.0 \\
\hline Rubus species & 7 & 0 & 0 & 0 & 7 & 39.8 \\
\hline Sassafras & 1 & 0 & 0 & 0 & 1 & 61.4 \\
\hline Blueberry & 39 & 1 & 0 & 0 & 40 & $16 \cdot 3$ \\
\hline Maple-leaved viburnum & 19 & 0 & 0 & 0 & 19 & $27 \cdot 3$ \\
\hline Arrowwood & 5 & 0 & 0 & 0 & 5 & 60.8 \\
\hline Other deciduous spectes & 36 & 0 & 0 & 0 & 36 & 23.7 \\
\hline Total deciduous species & 411 & 5 & 0 & 0 & 416 & 10.1 \\
\hline Unknown species & 9 & 0 & 0 & 0 & 9 & 33.4 \\
\hline Total, all species & 439 & 5 & 0 & 0 & 443 & 10.0 \\
\hline Sampling error & & & & & & \\
\hline (percent) & $10 \cdot 3$ & 88.9 & .0 & .0 & 10.0 & \\
\hline
\end{tabular}




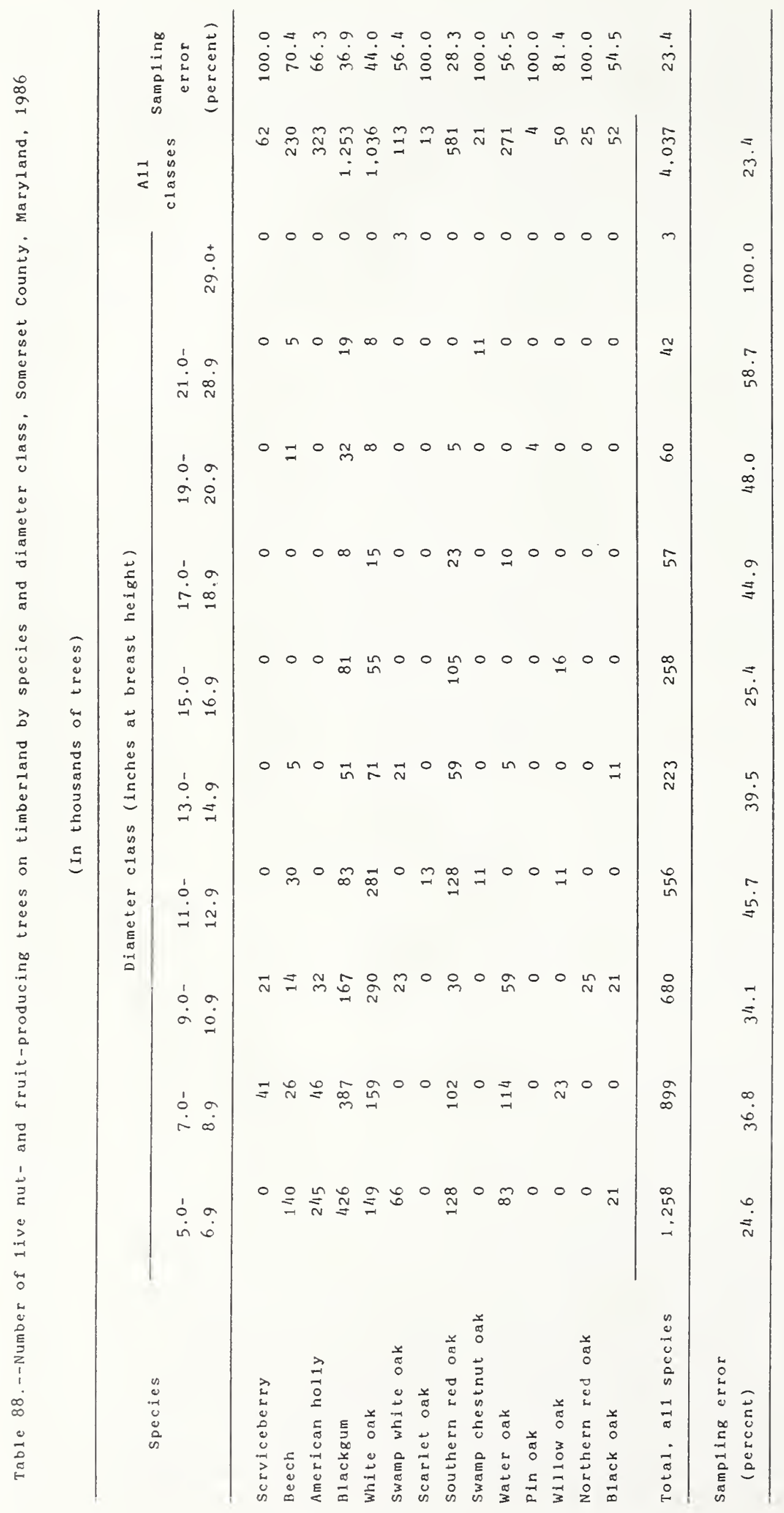







Table 90.--Number of seedlings, saplings, and shrubs on tiritrland by species and browse-utilization class, Somerset County. Maryland, 1986

( $1 \mathrm{n}$ millions of stems)

\begin{tabular}{|c|c|c|c|c|c|c|}
\hline \multirow{2}{*}{ Species } & \multicolumn{4}{|c|}{ Browse-utilization class } & \multirow{2}{*}{$\begin{array}{c}\text { All } \\
\text { classes }\end{array}$} & \multirow{2}{*}{$\begin{array}{c}\text { Sampling } \\
\text { error } \\
\text { (percent) }\end{array}$} \\
\hline & None & Light & Moderate & Heavy & & \\
\hline Loblolly pine & 50 & 0 & 0 & 0 & 50 & 44.9 \\
\hline Other softwoods & 1 & 0 & 0 & 0 & 1 & 100.0 \\
\hline Total coniferous species & 51 & 0 & 0 & 0 & 51 & 44.4 \\
\hline Red maple & $15 c$ & 11 & 0 & 0 & 162 & 28.9 \\
\hline Azalea species & 1 & 0 & 0 & 0 & 1 & 78.0 \\
\hline American beech & 0 & 0 & 0 & 0 & 0 & 100.0 \\
\hline Ash species & 1 & 0 & 0 & 0 & 1 & 100.0 \\
\hline American holly & 24 & 2 & 0 & 0 & 26 & 44.8 \\
\hline sweetgum & 51 & 4 & 0 & 0 & 54 & $27 \cdot 9$ \\
\hline Magnolia species & 3 & 0 & 0 & c & 3 & 100.0 \\
\hline Tupelo species & 38 & 0 & 0 & 0 & 38 & 50.9 \\
\hline Black cherry & 2 & $\mathrm{C}$ & 1 & 0 & 3 & $31 \cdot 1$ \\
\hline White oak & 1 & 1 & 0 & 0 & 2 & $61 \cdot 5$ \\
\hline Other white oaks & 2 & 0 & 0 & 0 & 2 & 80.8 \\
\hline Other black oaks & 13 & 0 & 0 & 0 & 13 & 66.4 \\
\hline Rose species & 1 & 0 & 0 & 0 & 1 & 100.0 \\
\hline Rubus species & 7 & 3 & 0 & 0 & 10 & $47 \cdot 3$ \\
\hline Sassafras & 6 & 0 & 0 & 0 & 6 & 47.0 \\
\hline Blueberry & 33 & 5 & 1 & 0 & 39 & 16.9 \\
\hline Maple-leaved viburnum & 0 & 1 & 0 & 0 & 1 & 100.0 \\
\hline Arrowwood & 1 & 0 & 0 & 0 & 1 & 100.0 \\
\hline Other deciduous species & 11 & 0 & 0 & 0 & 11 & $31 \cdot 7$ \\
\hline Total deciduous species & 344 & 27 & 2 & 0 & 373 & $17 \cdot 9$ \\
\hline Unknown species & 46 & 9 & 0 & 0 & 55 & $12 \cdot 3$ \\
\hline Total, a11 species & 441 & 36 & 2 & 0 & 480 & $17 \cdot 9$ \\
\hline Samp1ing error & & & & & & \\
\hline$($ percent) & 18.0 & 40.6 & $70 \cdot 9$ & .0 & $17 \cdot 9$ & \\
\hline
\end{tabular}












Table 93.--Number of seedlings, saplings, and shrubs on timberland by species and browse-utilization c1ass. Washington County. Maryland, 1986

(In millions of stems)

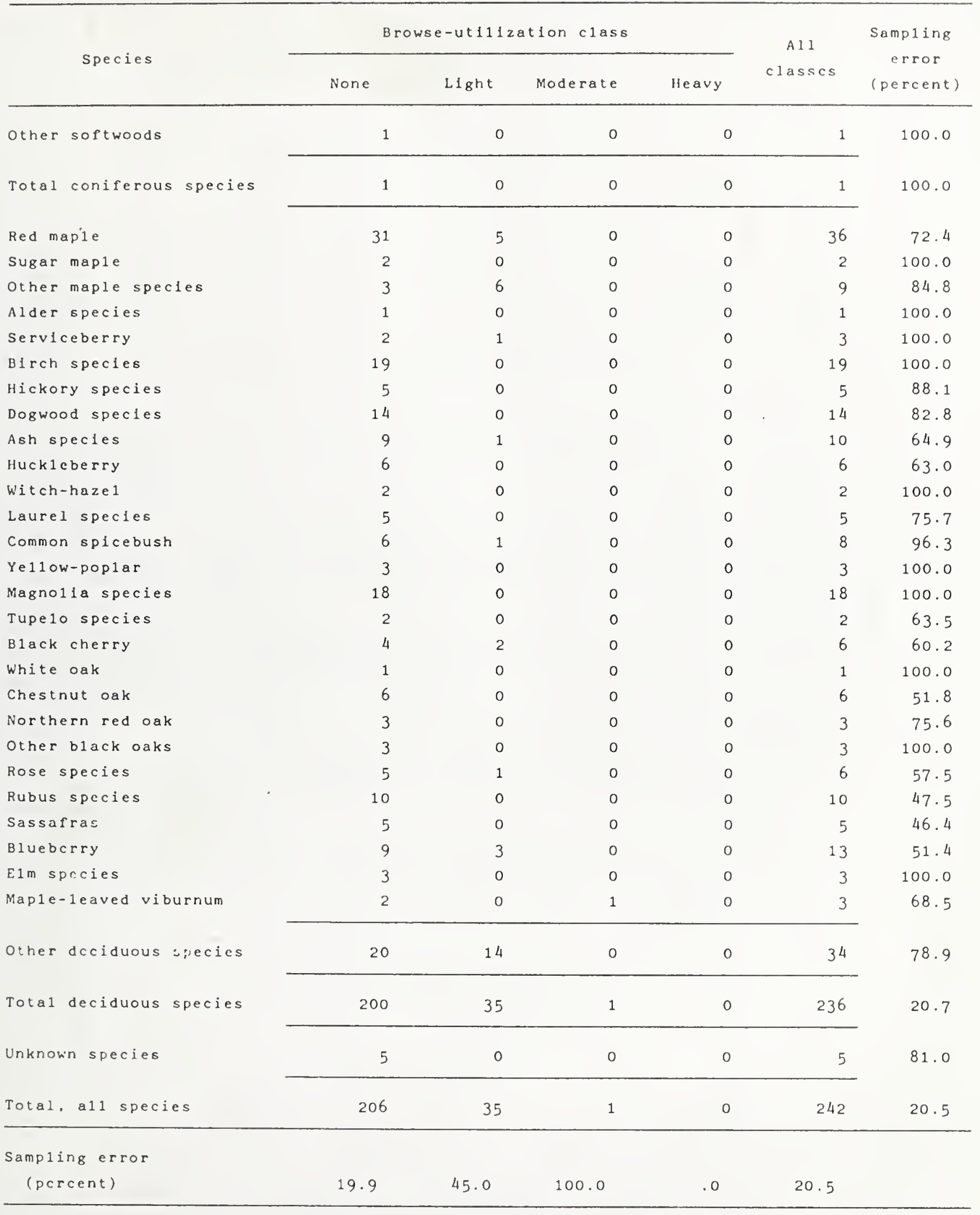









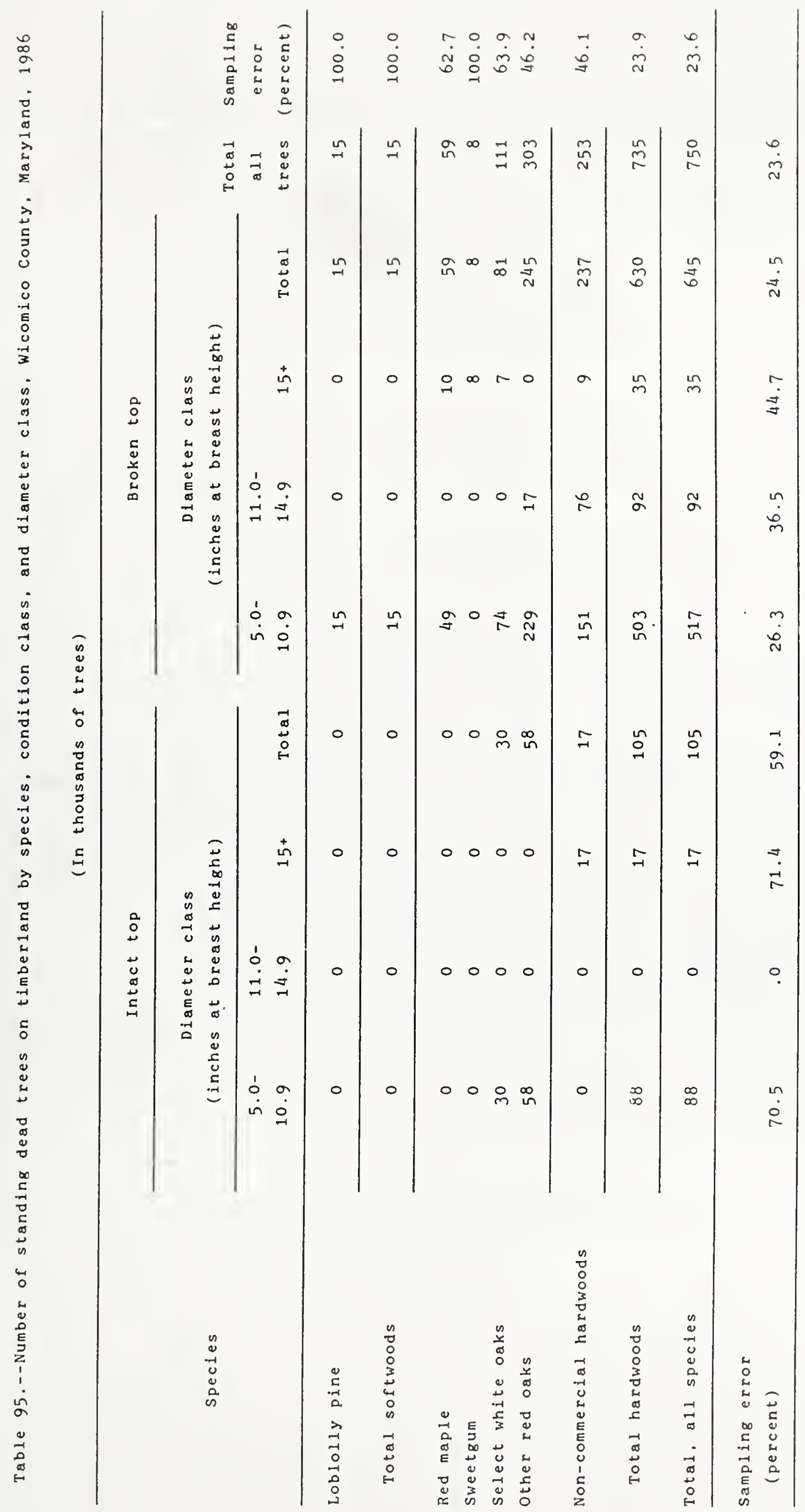


Table 96.--Number of seedlings, sa; lings, and shrubs on timberland by species and browse-utilization class, Wicomico County. Maryland, 1986

(In militions of stems)

\begin{tabular}{|c|c|c|c|c|c|c|}
\hline \multirow{2}{*}{ Species } & \multicolumn{4}{|c|}{ Browse-utilization class } & \multirow{2}{*}{$\begin{array}{c}\text { All } \\
\text { classes }\end{array}$} & \multirow{2}{*}{$\begin{array}{l}\text { Sampling } \\
\text { error } \\
\text { (percent) }\end{array}$} \\
\hline & None & Light & Moderate & Heavy & & \\
\hline Loblolly pine & 25 & 0 & 0 & 0 & 25 & 41.1 \\
\hline Other pine species & 0 & 0 & 0 & 0 & 0 & $100 \cdot 0$ \\
\hline Total coniferous species & 25 & 0 & 0 & 0 & 25 & 40.6 \\
\hline Red maple & 54 & 3 & 1 & 0 & 57 & 33.4 \\
\hline Serviceberry & 6 & 0 & 0 & 0 & 6 & 70.7 \\
\hline Azalea species & 2 & 0 & 0 & 0 & 2 & 59.9 \\
\hline American holly & 38 & 0 & 5 & 0 & 43 & 20.5 \\
\hline Laurel species & 1 & 0 & 0 & 0 & 1 & 100.0 \\
\hline Sweetgum & 61 & 2 & 0 & 0 & 63 & 25.7 \\
\hline Yellow-poplar & 1 & 0 & 0 & 0 & 1 & 100.0 \\
\hline Magnolia species & 6 & 5 & 0 & 0 & 11 & 48.6 \\
\hline Tupelo species & 14 & 2 & 0 & 0 & 16 & 37.6 \\
\hline Black cherry & 2 & 1 & 0 & 0 & 2 & 52.0 \\
\hline White oak & 2 & 0 & 3 & 0 & 5 & 70.1 \\
\hline Other black oaks & 28 & 1 & 0 & 0 & 29 & 34.6 \\
\hline Rubus species & 4 & 0 & 0 & 0 & 4 & 46.1 \\
\hline Sassafras & 10 & 1 & 2 & 0 & 13 & 38.4 \\
\hline Blueberry & 31 & 10 & 1 & 0 & 42 & $12 \cdot 3$ \\
\hline Arrowwood & 1 & 0 & 0 & 0 & 1 & 100.0 \\
\hline Other deciduous species & 7 & 2 & 0 & 0 & 10 & 29.4 \\
\hline Total deciduous species & 267 & 26 & 13 & 0 & 306 & 12.0 \\
\hline Unknown species & 81 & 17 & 3 & 0 & 100 & $18 \cdot 3$ \\
\hline Total, all species & 372 & 43 & 15 & 0 & 431 & $11 \cdot 7$ \\
\hline Sampling error & & & & & & \\
\hline$($ percent $)$ & 11.6 & 19.0 & 50.0 & .0 & 11.7 & \\
\hline
\end{tabular}




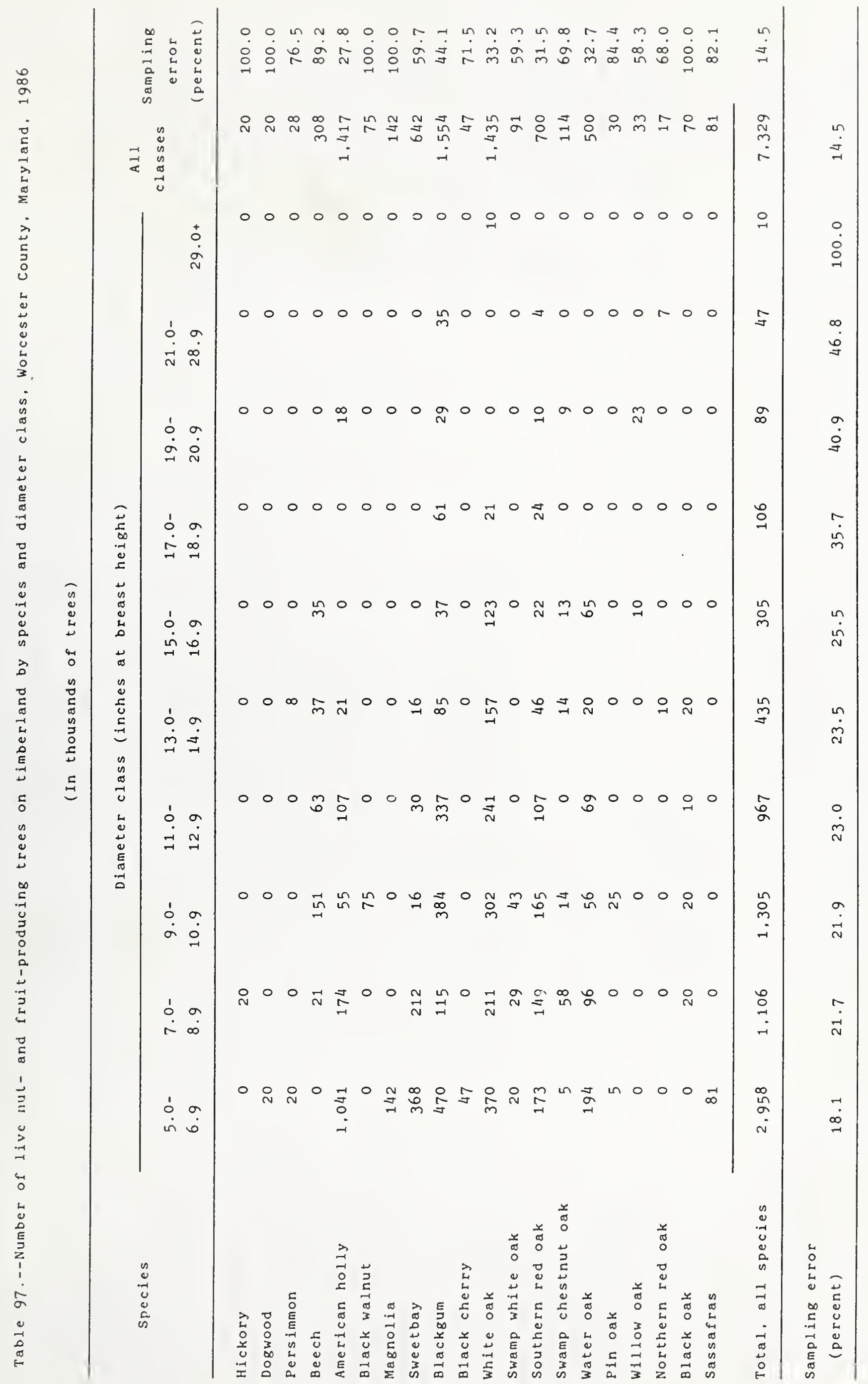




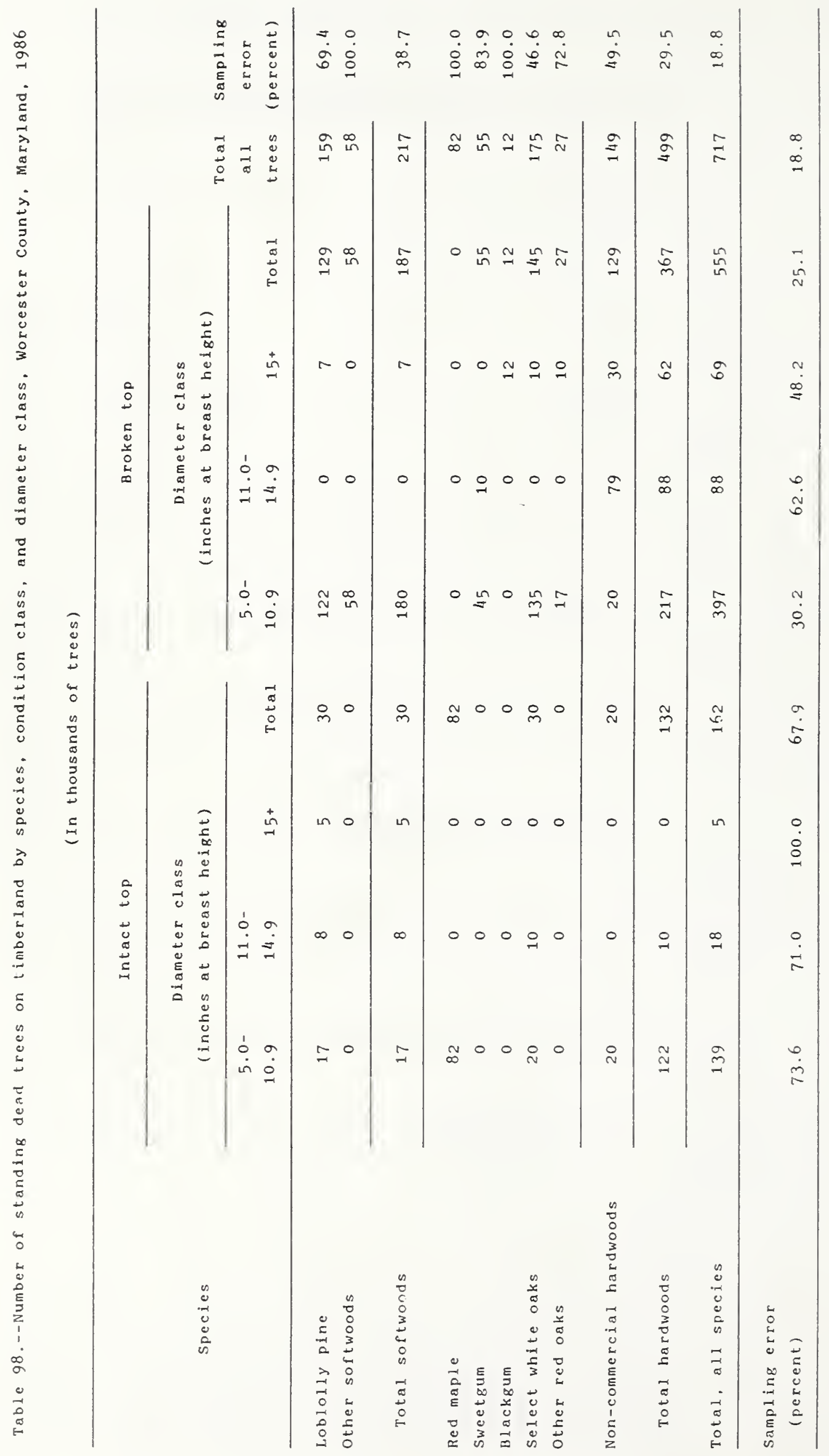


Table 99.--Number of seedlings, saplings, and shrubs on timberland by species and browse-utilization class, Worcester County, Maryland, 1986

(In millions of stems)

\begin{tabular}{|c|c|c|c|c|c|c|}
\hline \multirow{2}{*}{ Species } & \multicolumn{4}{|c|}{ Br: wse-utilization class } & \multirow{2}{*}{$\begin{array}{c}\text { All } \\
\text { classes }\end{array}$} & \multirow{2}{*}{$\begin{array}{l}\text { Sampling } \\
\text { error } \\
\text { (percent) }\end{array}$} \\
\hline & None & Light & Moderate & Heavy & & \\
\hline Lobloliy pine & 28 & 0 & 0 & 0 & 28 & 26.9 \\
\hline other softwoods & 1 & 0 & 0 & 0 & 1 & $74 \cdot 3$ \\
\hline Total coniferous species & 29 & 0 & 0 & 0 & 29 & 25.6 \\
\hline Red maple & 119 & 24 & 0 & 0 & 143 & $25 \cdot 5$ \\
\hline Serviceberry & 6 & 0 & 0 & 0 & 6 & $61 \cdot 3$ \\
\hline Azalea species & 13 & 1 & 2 & 0 & 15 & 30.4 \\
\hline Hickory species & 2 & 0 & 0 & 0 & 2 & 81.6 \\
\hline Dogwood species & 2 & 0 & 5 & 0 & 6 & 76.2 \\
\hline American beech & 1 & 0 & 0 & 0 & 1 & 74.4 \\
\hline Ash species & 2 & 0 & 0 & 0 & 2 & 100.0 \\
\hline American holly & 58 & 0 & 0 & 0 & 58 & 29.2 \\
\hline Laurel species & 2 & 0 & 0 & 0 & 2 & 70.8 \\
\hline Common spicebush & 2 & 0 & 0 & 0 & 2 & 71.8 \\
\hline Sweet gum & 64 & 10 & 0 & 0 & 75 & $23 \cdot 9$ \\
\hline Yellow-poplar & 8 & 0 & 0 & 0 & 8 & 82.1 \\
\hline Magnolia species & 33 & 0 & 0 & 0 & 33 & 56.8 \\
\hline Tupe 1o species & 22 & 5 & 0 & 0 & 27 & 24.0 \\
\hline Black cherry & 0 & 0 & 0 & 0 & 0 & 100.0 \\
\hline White oak & 4 & 1 & 0 & 0 & 5 & 44.8 \\
\hline Other white oaks & 2 & 0 & 0 & 0 & 2 & 71.0 \\
\hline Northern red oak & 0 & 0 & 0 & 0 & 0 & 100.0 \\
\hline Other black oaks & 37 & 8 & 3 & 0 & 48 & 34.8 \\
\hline Rose species & 1 & 0 & 0 & 0 & 1 & 73.4 \\
\hline Rubus species & 7 & 0 & 0 & 0 & 7 & 62.7 \\
\hline Sassafras & 11 & 3 & 2 & 0 & 16 & 41.0 \\
\hline Blueberry & 37 & 26 & 4 & 1 & 68 & $11 \cdot 7$ \\
\hline Elm species & 4 & 2 & 0 & 0 & 6 & 100.0 \\
\hline Arrowwood & 5 & 1 & 0 & 0 & 6 & 40.6 \\
\hline Other viburnum species & 1 & 0 & 0 & 0 & 1 & 100.0 \\
\hline Other deciduous species & 23 & 0 & 0 & 0 & 23 & $27 \cdot 4$ \\
\hline Total deciduous species & 465 & 82 & 17 & 1 & 565 & 12.5 \\
\hline Unknown species & 71 & 25 & 7 & 1 & 105 & 13.4 \\
\hline Total, all species & 566 & 107 & 24 & 1 & 699 & 10.5 \\
\hline Sampling error & & & & & & \\
\hline$($ percent) & 10.6 & 22.6 & 35.2 & 71.4 & 10.5 & \\
\hline
\end{tabular}




\section{DELAWARE}

STATE TABLES




Table 100.--Land area by county and land class. Delaware. 1986

\begin{tabular}{|c|c|c|c|}
\hline Land class & sussex & $\begin{array}{c}\text { Kent/ } \\
\text { New Castle }\end{array}$ & $\begin{array}{c}\text { All } \\
\text { countles }\end{array}$ \\
\hline TImberland & 217.6 & 158.8 & 376.4 \\
\hline \multicolumn{4}{|l|}{ Noncommercial forest land: } \\
\hline Product lve reserved & .2 & 2.7 & 2.9 \\
\hline Unproductive & 3.8 & 3.0 & 6.8 \\
\hline Urban & .0 & 3.4 & 3.4 \\
\hline Total forest & 221.6 & 167.9 & 389.5 \\
\hline \multicolumn{4}{|l|}{ Nonforest land: } \\
\hline Cropland & 258.2 & 250.5 & 508.7 \\
\hline Pasture & $12 \cdot 7$ & 12.2 & 24.9 \\
\hline Other Parmiand & 23.6 & $43 \cdot 3$ & 66.9 \\
\hline Other land & 86.7 & 160.0 & 246.7 \\
\hline Total nonforest & 381.2 & 466.0 & 847.2 \\
\hline Total land area & 602.8 & 633.9 & 1.236 .7 \\
\hline
\end{tabular}

a Source: 1982 Census of Agriculture.

Source: 1981 Unfted States Department of Commerce. Bureau of Census. 




a Edge condition on an aerial photograph sampled by a line transect (Brooks and sykes 1984). 





Table 103.--Area of timberland by forest type, forest-type group, and stand-size class, Delaware, 1986

(In thousands of acres)

\begin{tabular}{|c|c|c|c|c|c|}
\hline \multirow{2}{*}{ Forest type } & \multicolumn{4}{|c|}{ Stand-size class } & \multirow{2}{*}{$\begin{array}{c}\text { A11 } \\
\text { classes }\end{array}$} \\
\hline & Sawtimber & Poletimber & $\begin{array}{l}\text { sapling and } \\
\text { seedling }\end{array}$ & Nonstocked & \\
\hline Loblolly pine & 40.5 & 10.1 & 24.5 & .0 & 75.1 \\
\hline Virginia pine & $5 \cdot 9$ & .0 & .0 & .0 & 5.9 \\
\hline Loblolly/shortleap group & 46.4 & 10.1 & $24 \cdot 5$ & .0 & 81.0 \\
\hline Virginia pine/oak & 3.9 & 6.9 & 5.1 & .0 & $15 \cdot 9$ \\
\hline Loblolly pine/hardwood & 38.3 & $3 \cdot 9$ & 2.2 & .0 & 44.4 \\
\hline Oak/pine group & 42.2 & 10.8 & $7 \cdot 4$ & .0 & $60 \cdot 3$ \\
\hline White oak & $13 \cdot 5$ & 9.9 & .0 & .0 & 23.4 \\
\hline Northern red oak & 2.7 & .0 & $3 \cdot 9$ & .0 & 6.5 \\
\hline Y. poplar/wh. oak/no. red oak & 3.0 & .0 & .0 & .0 & 3.0 \\
\hline Sweetgum/yellow-poplar & 5.0 & .0 & 10.2 & .0 & 15.2 \\
\hline Yellow-poplar & 5.9 & .0 & .0 & .0 & $5 \cdot 9$ \\
\hline Scarlet oak & 6.9 & $3 \cdot 9$ & 3.8 & .0 & 14.6 \\
\hline Red maple/central hardwoods & 2.7 & 3.0 & .0 & .0 & 5.6 \\
\hline Mixed central hardwoods & 52.6 & 18.4 & 11.8 & .0 & 82.8 \\
\hline Oak/hickory group & $92 \cdot 3$ & 35.1 & 29.6 & .0 & 157.1 \\
\hline Swamp chstnt oak/cherrybark oak & 5.0 & .0 & .0 & .0 & 5.0 \\
\hline Sweetgum/nuttall oak/willow oak & 27.2 & 9.9 & .0 & .0 & 37.0 \\
\hline Sweetbay/swamp tupelo/red maple & 6.9 & $3 \cdot 9$ & 5.0 & .0 & 15.8 \\
\hline Oak/gum/cypress group & 39.1 & 13.8 & 5.0 & .0 & $57 \cdot 9$ \\
\hline Black ash/Amer. elm/red maple & .0 & .0 & 2.2 & .0 & 2.2 \\
\hline Red maple (lowland) & 5.0 & .0 & .0 & .0 & 5.0 \\
\hline American elm/green ash & 3.0 & .0 & .0 & .0 & 3.0 \\
\hline Elm/ash/red maple group & 8.0 & .0 & 2.2 & .0 & $10 \cdot 3$ \\
\hline Black cherry & 6.9 & .0 & .0 & .0 & 6.9 \\
\hline Mixed northern hardwoods & 3.0 & .0 & .0 & .0 & 3.0 \\
\hline Northern hardwoods group & 9.8 & .0 & .0 & .0 & 9.8 \\
\hline All forest types & 237.9 & 69.7 & 68.8 & .0 & 376.4 \\
\hline
\end{tabular}


Table 104.--Area of timberland by county and stand-area c1ass, Delaware, 1986

(In thousands of acres)

\begin{tabular}{|c|c|c|c|c|c|c|c|}
\hline \multirow{2}{*}{ County } & \multicolumn{6}{|c|}{ Stand-area c1ass } & \multirow{2}{*}{$\begin{array}{c}\text { All } \\
\text { classes }\end{array}$} \\
\hline & $1-9$ & $10-19$ & $20-49$ & $50-99$ & $100-499$ & $500+$ & \\
\hline Sussex & 30.6 & 75.8 & 98.5 & 17.8 & 8.8 & .0 & 231.6 \\
\hline Kent/New Castle & $32 \cdot 3$ & 25.8 & 51.2 & 23.1 & $12 \cdot 5$ & .0 & 144.8 \\
\hline State Total & 62.9 & 101.7 & 149.7 & 40.8 & 21.3 & .0 & 376.4 \\
\hline
\end{tabular}







Table 106.--Number of shrubs and saplings on timberland by stand-size class, type of stem, and mast type. Delaware, 1986

(In thousands of stems)

\begin{tabular}{|c|c|c|c|c|c|}
\hline \multirow{2}{*}{$\begin{array}{l}\text { Stand-size class } \\
\text { and type of stem }\end{array}$} & \multicolumn{4}{|c|}{ Mast type } & \multirow{2}{*}{$\begin{array}{l}\text { Total } \\
\text { stems }\end{array}$} \\
\hline & Nuts & Other & Berries & $\begin{array}{l}\text { Other } \\
\text { species }\end{array}$ & \\
\hline \multicolumn{6}{|l|}{ Sawt Imber: } \\
\hline Shrubs & 681 & 15.244 & 116,120 & 138.650 & 270.695 \\
\hline Saplings & 5.834 & 45.031 & 59.576 & 0 & 110,442 \\
\hline Total sawtimber & 6.515 & 60.275 & 175.696 & 138.650 & 381.137 \\
\hline \multicolumn{6}{|l|}{ Poletimber: } \\
\hline Shrubs & 0 & 7.118 & 31.992 & 45.198 & 84.308 \\
\hline Saplings & 7.385 & 36.012 & 8.707 & 0 & 52.103 \\
\hline Total poletimber & 7.385 & 43.130 & 40.698 & 45.198 & 136.411 \\
\hline \multicolumn{6}{|l|}{ Sapling/seedling: } \\
\hline Shrubs & 0 & 4.549 & 51.003 & 38.069 & 93.622 \\
\hline Saplings & 4.707 & 44.956 & 4,133 & 0 & 53.796 \\
\hline Total sapling/seedling & 4.707 & 49.506 & 55.136 & 38.069 & 147.418 \\
\hline Total, all classes & 18.608 & 152.911 & 271.530 & 221.917 & 664.967 \\
\hline
\end{tabular}




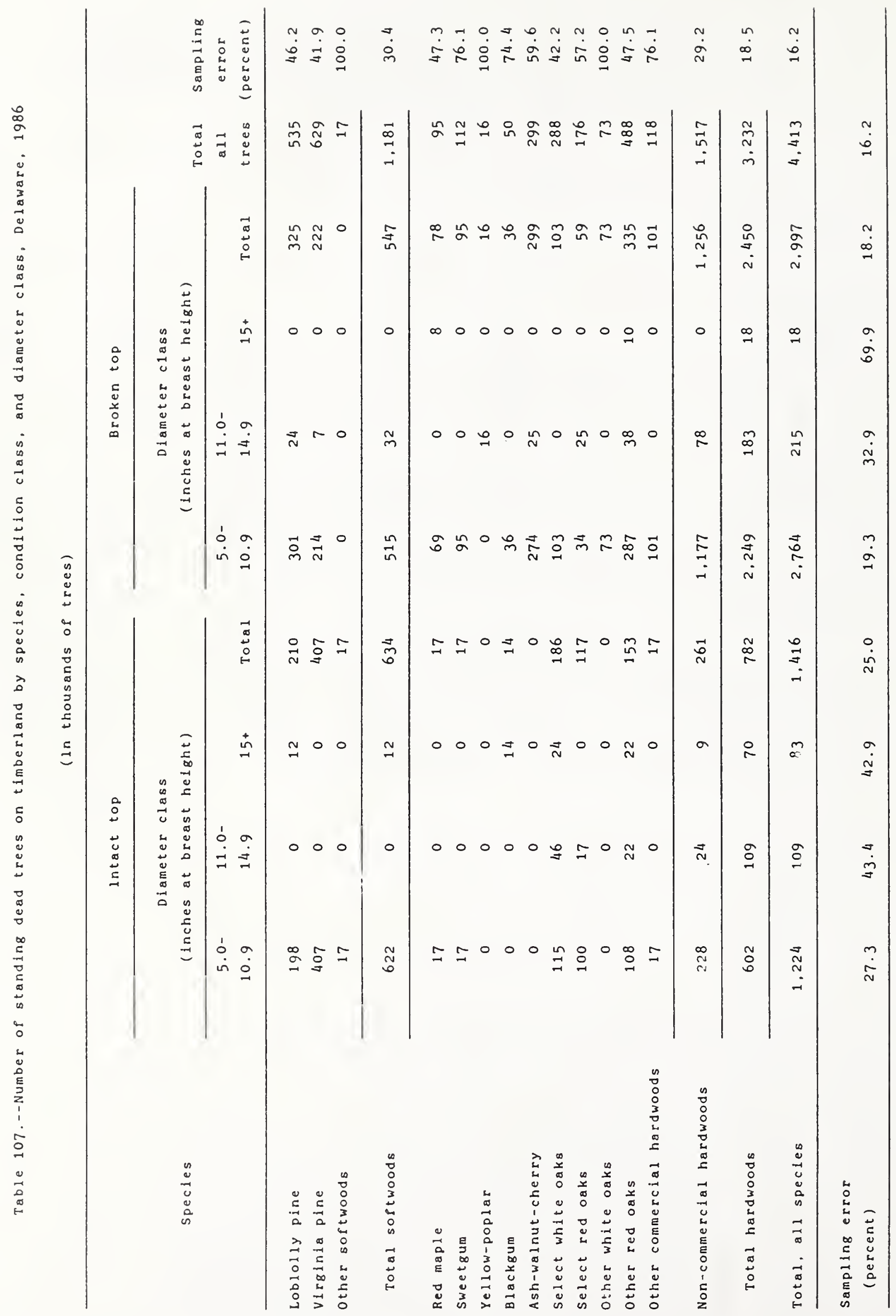




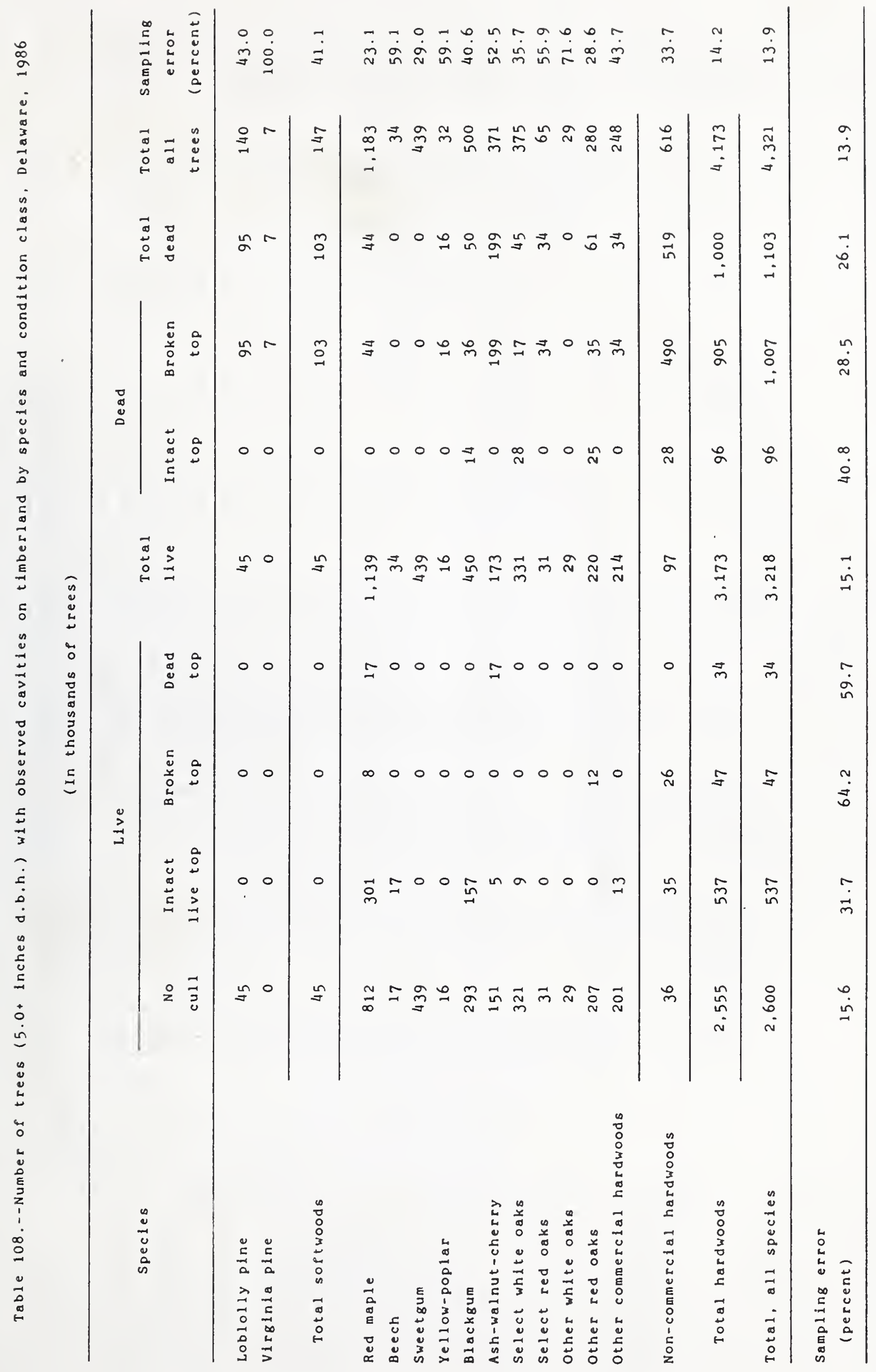




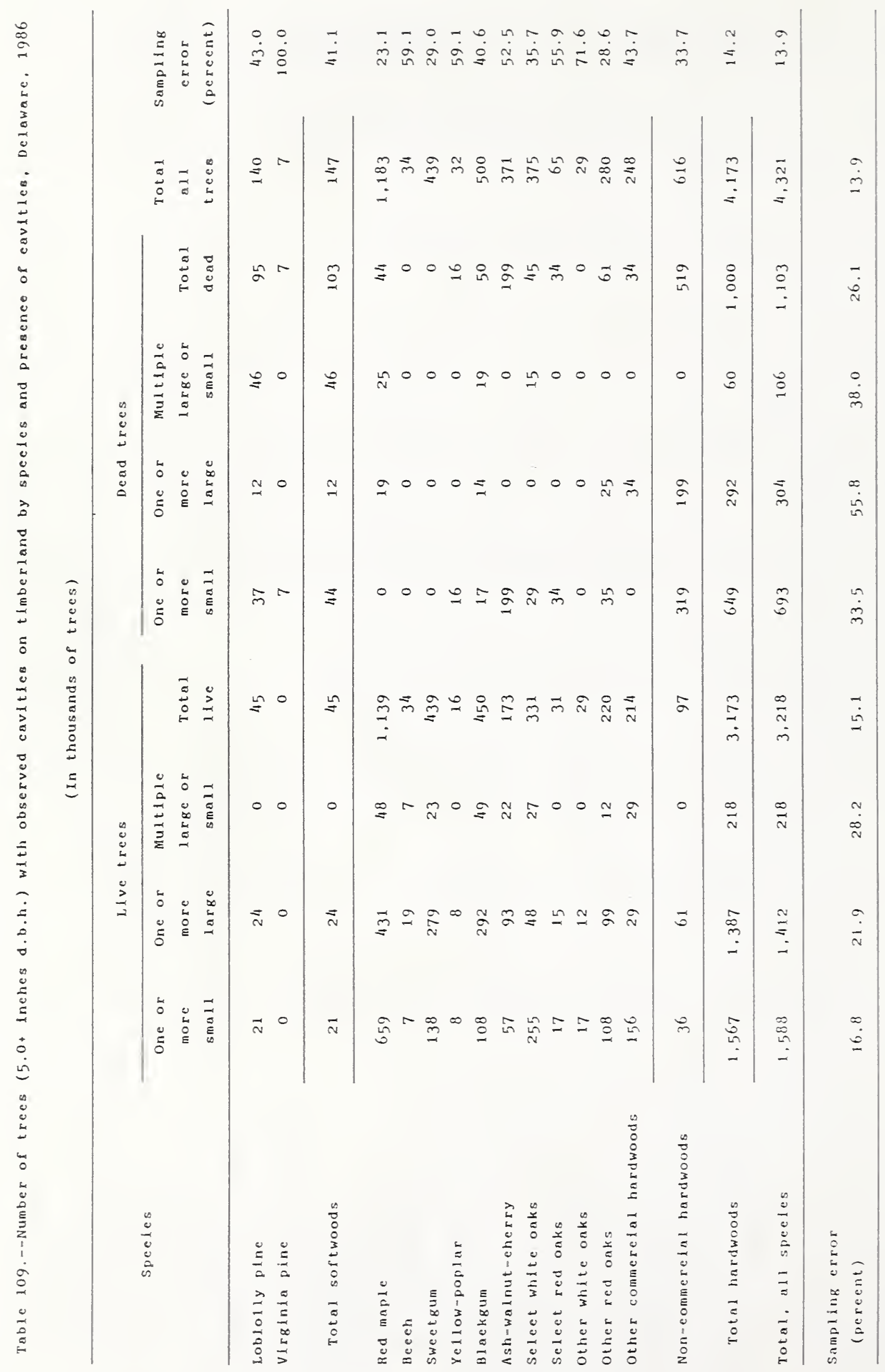


Table 110.- Number of seedlings, saplings, and shrubs on timberland by species and stand-size class. Delaware, 1986

(In millions of stems)

\begin{tabular}{|c|c|c|c|c|c|c|}
\hline \multirow{2}{*}{ Species } & \multicolumn{4}{|c|}{ Stand-size class } & \multirow{2}{*}{$\begin{array}{c}\text { All } \\
\text { classes }\end{array}$} & \multirow{2}{*}{$\begin{array}{c}\text { Sampling } \\
\text { error } \\
\text { (percent) }\end{array}$} \\
\hline & Sawtimber & Poletimber & seed 1 ing & Nonstocked & & \\
\hline Lob1ol1y pine & 7 & 11 & 57 & 0 & 75 & 28.2 \\
\hline Other pine specles & 11 & 4 & 3 & 0 & 18 & 43.8 \\
\hline Other softwoods & 1 & 1 & 0 & 0 & 3 & 48.8 \\
\hline Total coniferous species & 19 & 16 & 60 & 0 & 96 & 24.2 \\
\hline Red maple & 105 & 76 & 143 & 0 & 324 & $18 \cdot 3$ \\
\hline Sugar maple & 0 & 1 & 0 & 0 & 1 & 100.0 \\
\hline Other maple species & 11 & 0 & 2 & 0 & 13 & 60.7 \\
\hline Alder species & 1 & 0 & 0 & 0 & 1 & 100.0 \\
\hline Serviceberry & 6 & 7 & 0 & 0 & 13 & 48.5 \\
\hline Azalea species & 11 & 7 & 2 & 0 & 20 & 26.6 \\
\hline Common pawpaw & 29 & 0 & 0 & 0 & 29 & 100.0 \\
\hline Hickory species & 4 & 1 & 4 & 0 & 10 & 38.7 \\
\hline Dogwood species & 31 & 2 & 5 & 0 & 39 & 36.1 \\
\hline American beech & 9 & 0 & 3 & 0 & 12 & 36.6 \\
\hline Ash species & 1 & 0 & 0 & 0 & 1 & 100.0 \\
\hline Witch-haze 1 & 1 & 0 & 1 & 0 & 1 & $74 \cdot 3$ \\
\hline American ho11y & 94 & 26 & 17 & 0 & 138 & 21.1 \\
\hline Laurel species & 3 & 0 & 0 & 0 & 3 & 74.9 \\
\hline Common spicebush & 11 & 0 & 0 & 0 & 11 & 41.6 \\
\hline Sweetgum & 86 & 47 & 65 & 0 & 199 & 20.9 \\
\hline Ye11ow-pop1ar & 17 & 0 & 21 & 0 & 37 & 43.8 \\
\hline Magnolia species & 41 & 4 & 2 & 0 & 47 & 59.1 \\
\hline Tupelo species & 46 & 19 & 34 & 0 & 99 & $21 \cdot 1$ \\
\hline Black cherry & 44 & 2 & 70 & 0 & 115 & 50.0 \\
\hline Other cherry species & 0 & 0 & 1 & 0 & 1 & 100.0 \\
\hline White oak & 11 & 2 & 8 & 0 & 21 & $26 \cdot 3$ \\
\hline Chestnut oak & 3 & 0 & 0 & 0 & 3 & 48.8 \\
\hline Other white oaks & 3 & 1 & 0 & 0 & 4 & 69.6 \\
\hline Northern red oak & 5 & 1 & 2 & 0 & 8 & 56.9 \\
\hline other black oaks & 43 & 14 & 28 & 0 & 84 & 25.8 \\
\hline Rose species & 2 & 0 & 3 & 0 & 5 & 67.4 \\
\hline Rubus species & 13 & 3 & 18 & 0 & 35 & $22 \cdot 9$ \\
\hline Sassafras & 17 & $1 \hat{j}$ & 67 & 0 & 97 & $27 \cdot 3$ \\
\hline Blueberry & 49 & 23 & 19 & 0 & 91 & 13.2 \\
\hline Elm species & 5 & 1 & 0 & 0 & 6 & 61.4 \\
\hline Maple-leaved viburnum & 5 & 0 & 0 & 0 & 5 & 48.6 \\
\hline Arrowwood & 26 & 5 & 4 & 0 & 34 & 19.1 \\
\hline Other viburnum species & 5 & 0 & 0 & 0 & 5 & $52 \cdot 5$ \\
\hline Other deciduous species & 18 & 2 & 18 & 0 & 38 & 22.0 \\
\hline Total deciduous species & 756 & 257 & 537 & 0 & 1.549 & 10.2 \\
\hline Unknown species & 133 & 43 & 28 & 0 & 205 & 10.2 \\
\hline Total, all species & 908 & 316 & 625 & 0 & 1.850 & 9.0 \\
\hline $\begin{array}{l}\text { Sampling error } \\
\text { (percent) }\end{array}$ & 12.1 & 24.9 & $27 \cdot 3$ & .0 & 9.0 & \\
\hline
\end{tabular}









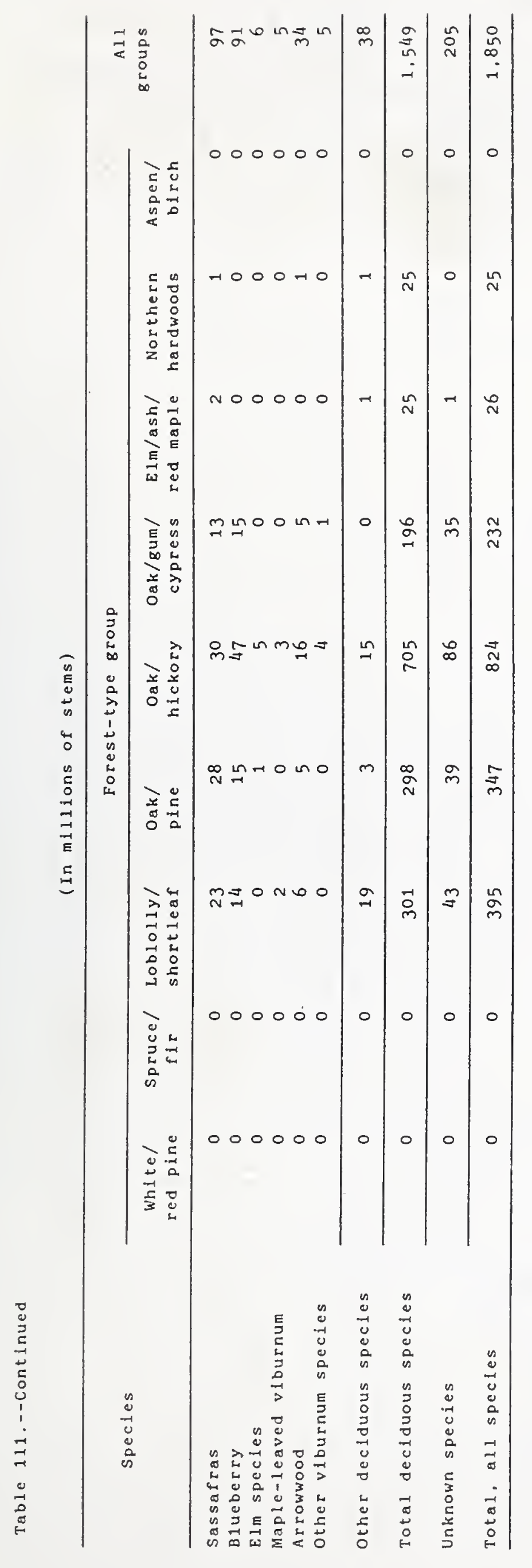


Table 112.--Number of seedilngs, saplings, and shrubs on timberland by species and browse-utilization class, Delaware, 1986

(1n milions of stems)

\begin{tabular}{|c|c|c|c|c|c|c|}
\hline \multirow{2}{*}{ Species } & \multicolumn{4}{|c|}{ Browse-utilization class } & \multirow{2}{*}{$\begin{array}{c}\text { All } \\
\text { classes }\end{array}$} & \multirow{2}{*}{$\begin{array}{l}\text { Sampling } \\
\text { error } \\
\text { (percent) }\end{array}$} \\
\hline & None & Light & Moderate & Heavy & & \\
\hline Lob1ol1y pine & 75 & 0 & 0 & 0 & 75 & 28.2 \\
\hline Other pine species & 18 & 0 & 0 & 0 & 18 & 43.8 \\
\hline other softwoods & 3 & 0 & 0 & 0 & 3 & 48.8 \\
\hline Total coniferous species & 96 & 0 & 0 & 0 & 96 & 24.2 \\
\hline Red maple & 309 & 7 & 7 & 1 & 324 & $18 \cdot 3$ \\
\hline Sugar maple & 1 & 0 & 0 & 0 & 1 & 100.0 \\
\hline other maple species & 2 & 7 & 4 & 0 & 13 & 60.7 \\
\hline Alder species & 1 & 0 & 0 & 0 & 1 & 100.0 \\
\hline Serviceberry & 11 & 2 & 0 & 0 & 13 & 48.5 \\
\hline Azalea species & 18 & 1 & 1 & 0 & 20 & 26.6 \\
\hline Common pawpaw & 29 & 0 & 0 & 0 & 29 & 100.0 \\
\hline Hickory species & 10 & 0 & 1 & 0 & 10 & 38.7 \\
\hline Dogwood species & 30 & 3 & 3 & 1 & 39 & 36.1 \\
\hline American beech & 9 & 1 & 1 & 0 & 12 & 36.6 \\
\hline Ash species & 1 & 0 & 0 & 0 & 1 & 100.0 \\
\hline Witch-haze 1 & 1 & 0 & 0 & 0 & 1 & $74 \cdot 3$ \\
\hline American holly & 138 & 0 & 0 & 0 & 138 & 21.1 \\
\hline Laure 1 species & 3 & 0 & 0 & 0 & 3 & 74.9 \\
\hline Common spicebush & 9 & 2 & 1 & 0 & 11 & 41.6 \\
\hline Sweetgum & 194 & 3 & 1 & 1 & 199 & 20.9 \\
\hline Ye11ow-pop1ar & 36 & 0 & 1 & 0 & 37 & 43.8 \\
\hline Magnolia species & 47 & 0 & 0 & 0 & 47 & 59.1 \\
\hline Tupelo species & 88 & 11 & 1 & 0 & 99 & 21.1 \\
\hline Black cherry & 104 & 6 & 4 & 1 & 115 & 50.0 \\
\hline Other cherry species & 1 & 0 & 0 & 0 & 1 & 100.0 \\
\hline White oak & 18 & 3 & 0 & 0 & 21 & $26 \cdot 3$ \\
\hline Chestnut oak & 2 & 1 & 0 & 0 & 3 & 48.8 \\
\hline Other white oaks & 4 & 0 & 0 & 0 & 4 & 69.6 \\
\hline Northern red oak & 7 & 1 & 0 & 0 & 8 & 56.9 \\
\hline other black oaks & 72 & 11 & 1 & 0 & 84 & 25.8 \\
\hline Rose species & 5 & 0 & 0 & 0 & 5 & 67.4 \\
\hline Rubus species & 35 & 0 & 0 & 0 & 35 & 22.9 \\
\hline Sassafras & 89 & 5 & 2 & 2 & 97 & $27 \cdot 3$ \\
\hline B1ueberry & 69 & 16 & 5 & 1 & 91 & 13.2 \\
\hline E1m species & 6 & 0 & 0 & 0 & 6 & 61.4 \\
\hline Map1e-1 eaved viburnum & 4 & 0 & 1 & 0 & 5 & 48.6 \\
\hline Arrowwood & 26 & 2 & 4 & 1 & 34 & 19.1 \\
\hline Other viburnum species & 4 & 0 & 1 & 0 & 5 & 52.5 \\
\hline other deciduous species & 32 & 6 & 1 & 0 & 38 & 22.0 \\
\hline Tota 1 deciduous species & 1.413 & 87 & 41 & 8 & 1.549 & 10.2 \\
\hline Unknown species & 171 & 21 & 11 & 1 & 205 & 10.2 \\
\hline Tota1, a11 species & 1,680 & 108 & 52 & 10 & 1,850 & 9.0 \\
\hline $\begin{array}{l}\text { Sampling error } \\
\text { (percent) }\end{array}$ & 9.8 & 14.5 & 21.6 & 49.6 & 9.0 & \\
\hline
\end{tabular}




\section{DELAWARE COUNTY TABLES}



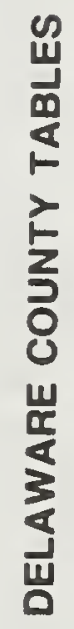









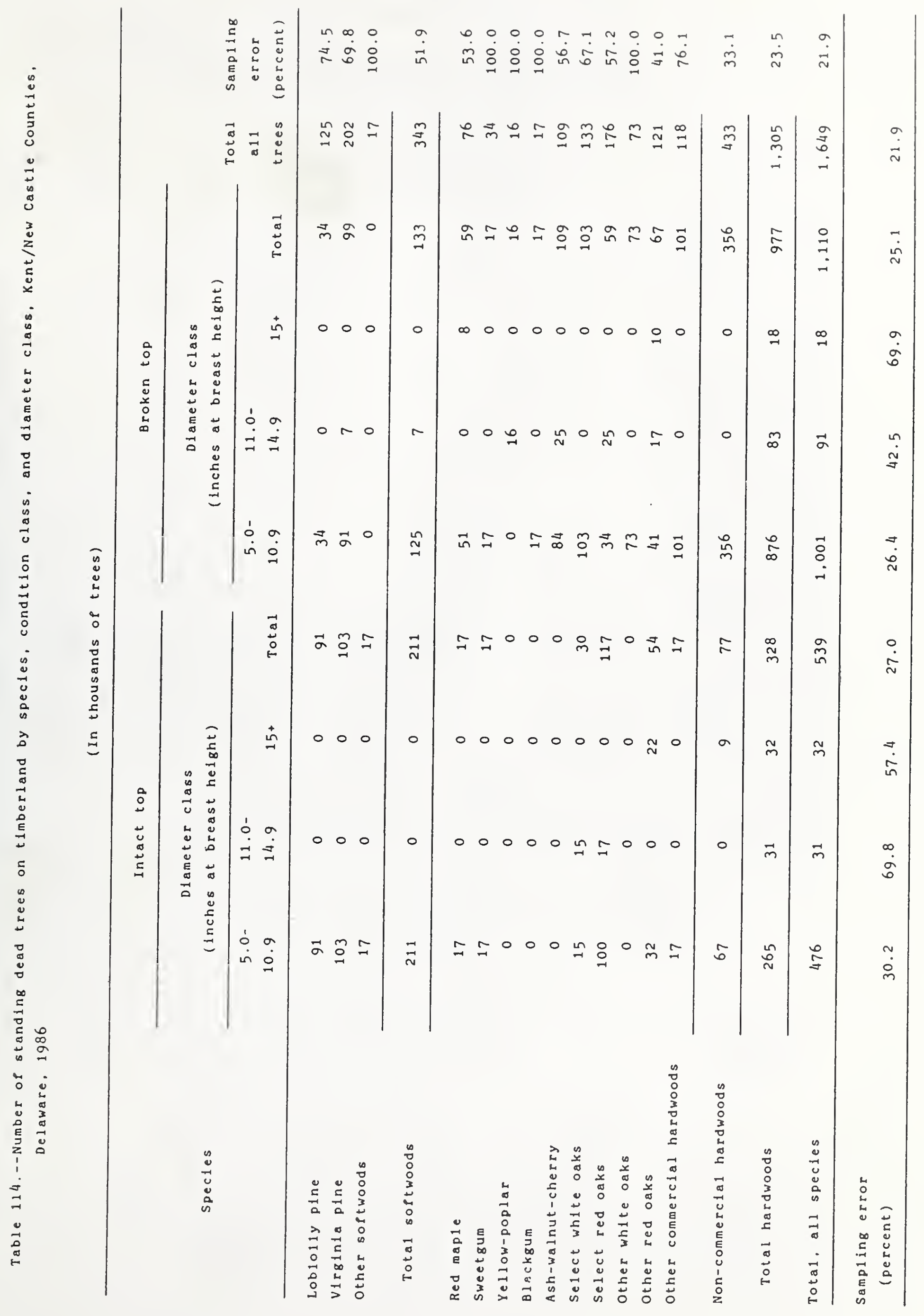


Table 115.--Number of seedlings, saplings, and shrubs on timberland by species and browse-utilization class, Kent/New Castle Counties.

Delaware, 1986

(In millions of stems)

\begin{tabular}{|c|c|c|c|c|c|c|}
\hline \multirow{2}{*}{ Species } & \multicolumn{4}{|c|}{ Browse-utilization class } & \multirow{2}{*}{$\begin{array}{c}\text { A } 11 \\
\text { c lasses }\end{array}$} & \multirow{2}{*}{$\begin{array}{l}\text { Sampling } \\
\text { error } \\
\text { (percent) }\end{array}$} \\
\hline & None & Light & Moderate & Heavy & & \\
\hline Loblolly pine & 9 & 0 & 0 & 0 & 9 & 86.0 \\
\hline Other softwoods & 3 & 0 & 0 & 0 & 3 & 48.8 \\
\hline Total coniferous species & 12 & 0 & 0 & 0 & 12 & 67.8 \\
\hline Red maple & 67 & 1 & 5 & 1 & 73 & 28.2 \\
\hline Sugar maple & 1 & 0 & 0 & 0 & 1 & 100.0 \\
\hline Other maple specles & 2 & 7 & 4 & 0 & 13 & 60.7 \\
\hline Serviceberry & 11 & 0 & 0 & 0 & 11 & 54.4 \\
\hline Azalea species & 11 & 1 & 1 & 0 & 13 & 30.2 \\
\hline Common pawpaw & 29 & 0 & 0 & 0 & 29 & 100.0 \\
\hline Hickory species & 5 & 0 & 1 & 0 & 5 & 61.0 \\
\hline Dogwood specles & 22 & 1 & 3 & 1 & 28 & $45 \cdot 7$ \\
\hline American beech & 5 & 1 & 1 & 0 & 7 & 49.1 \\
\hline Ash species & 1 & 0 & 0 & 0 & 1 & 100.0 \\
\hline Witch-hazel & 1 & 0 & 0 & 0 & 1 & 100.0 \\
\hline American holly & 30 & 0 & 0 & 0 & 30 & 33.8 \\
\hline Laurel specles & 2 & 0 & 0 & 0 & 2 & 100.0 \\
\hline Common splcebush & 9 & 2 & 1 & 0 & 11 & 41.6 \\
\hline sweetgum & 50 & 3 & 1 & 1 & 55 & 20.1 \\
\hline Yel low-poplar & 20 & 0 & 1 & 0 & 21 & 45.2 \\
\hline Magnolia specles & 5 & 0 & 0 & 0 & 5 & 46.5 \\
\hline Tupe 10 species & 37 & 9 & 1 & 0 & 47 & 24.4 \\
\hline Black cherry & 20 & 5 & 4 & 1 & 30 & $37 \cdot 5$ \\
\hline White oak & 7 & 3 & 0 & 0 & 10 & 42.4 \\
\hline Chestnut oak & 2 & 1 & 0 & 0 & 3 & 48.8 \\
\hline other white oaks & 4 & 0 & 0 & 0 & 4 & 69.6 \\
\hline Northern red oak & 1 & 1 & 0 & 0 & 2 & 57.2 \\
\hline Other black oaks & 8 & 2 & 1 & 0 & 11 & 43.5 \\
\hline Rose species & 5 & 0 & 0 & 0 & 5 & 67.4 \\
\hline Rubus species & 15 & 0 & 0 & 0 & 15 & 26.5 \\
\hline Sassafras & 19 & 5 & 2 & 2 & 27 & $41 \cdot 5$ \\
\hline Blueberry & 29 & 9 & 3 & 1 & 42 & 19.7 \\
\hline Elm species & 6 & 0 & 0 & 0 & 6 & 61.4 \\
\hline Maple-leaved viburnum & 4 & 0 & 1 & 0 & 5 & 48.6 \\
\hline Arrowwood & 19 & 1 & 3 & 1 & 25 & $22 \cdot 5$ \\
\hline Other viburnum species & 4 & 0 & 1 & 0 & 5 & $52 \cdot 5$ \\
\hline other deciduous species & 10 & 1 & 1 & 0 & 12 & 29.1 \\
\hline Total deciduous species & 459 & 54 & 35 & 8 & 556 & 11.0 \\
\hline Unknown species & 57 & 9 & 9 & 0 & 75 & $17 \cdot 3$ \\
\hline Total, all species & 528 & 63 & 43 & 8 & 643 & 10.0 \\
\hline Sampling error & & & & & & \\
\hline$($ percent $)$ & 11.4 & 20.9 & $25 \cdot 1$ & $55 \cdot 7$ & 10.0 & \\
\hline
\end{tabular}




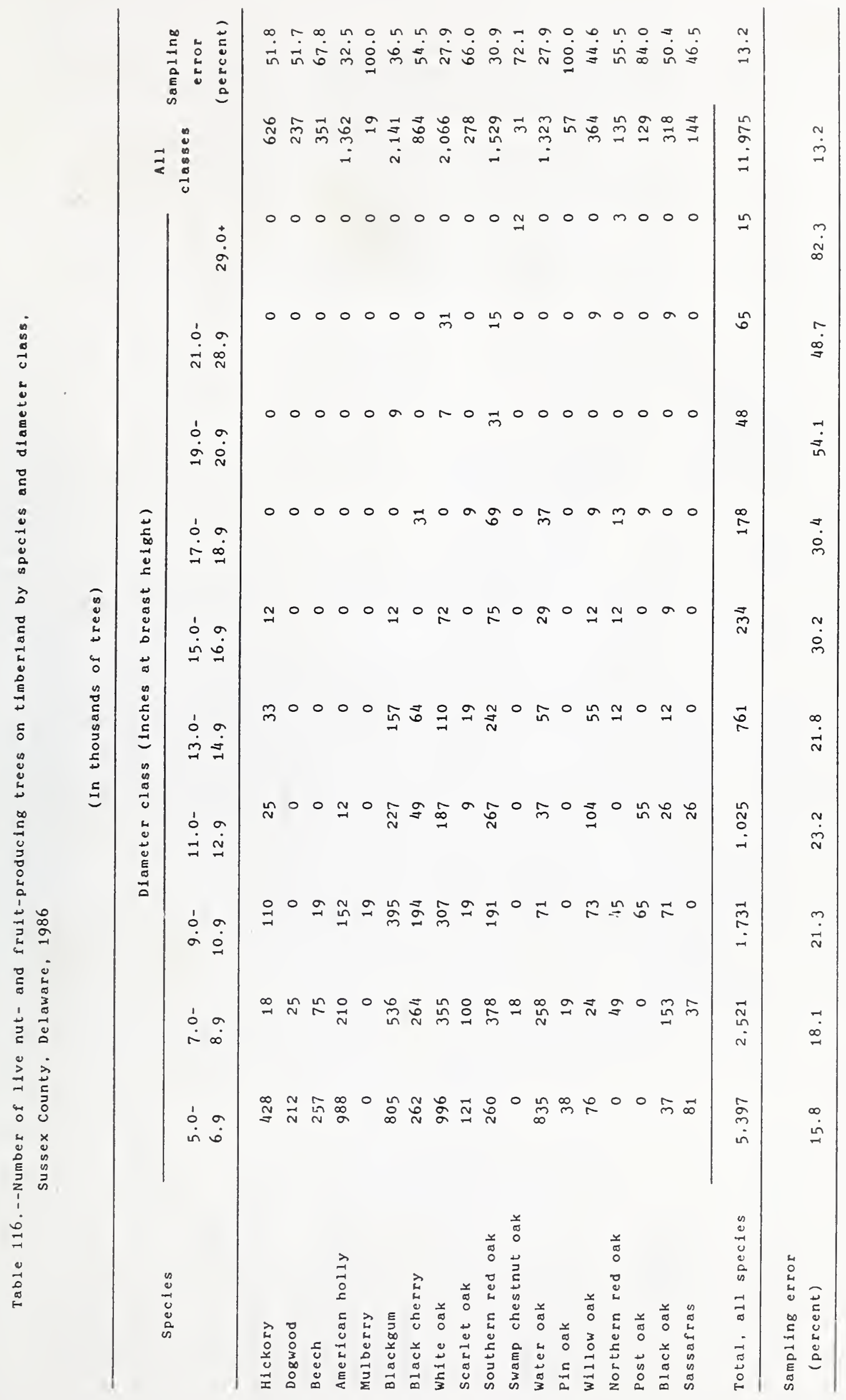




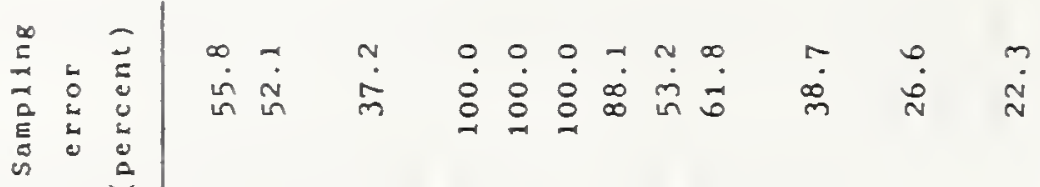


$\mid$

葛

$\frac{5}{2}$

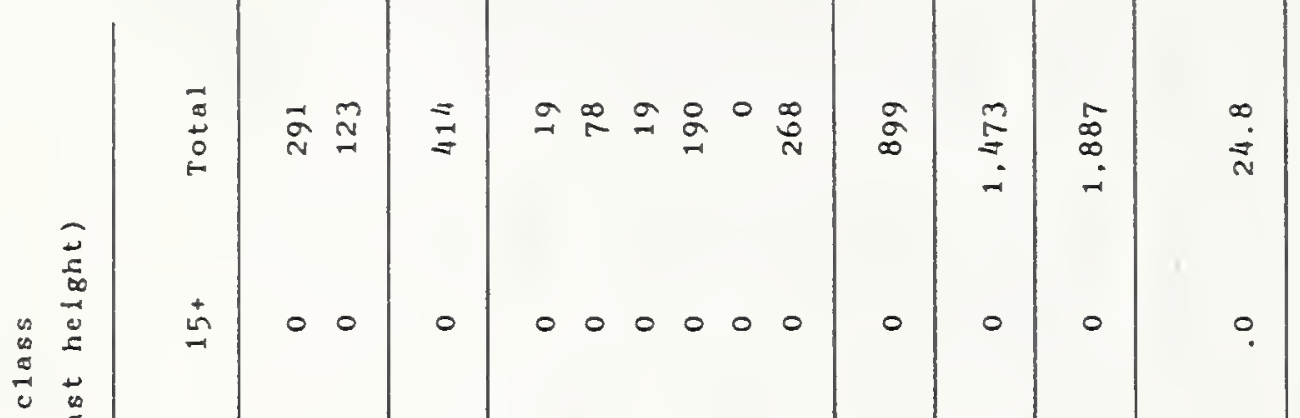


Table 118.--Number of seedlings, seplings, and shrubs on timberland by species and browse-utilization class, sussex county.

Delaware, 1986

(In millons of stems)

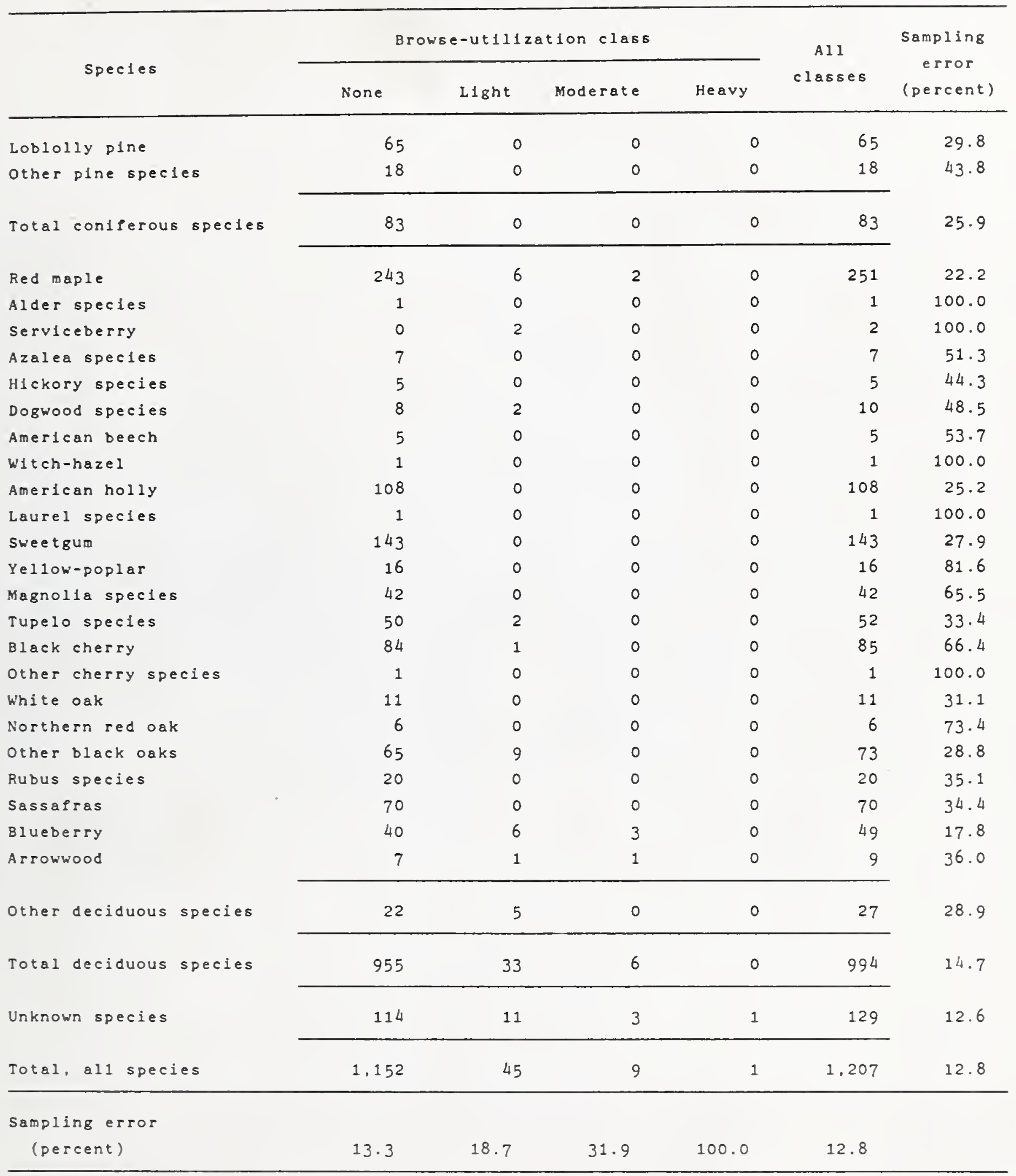



Brooks, Robert T.; DiGiovanni, Dawn M. 1989. Forest wildlife habitat statistics for Maryland and Delaware--1986. Res. Bull. NE-110. Broomall, PA: U.S. Department of Agriculture, Forest Service, Northeastern Forest Experiment Station. $171 \mathrm{p}$.

A statistical report on the forest wildlife habitat survey of Maryland and Delaware (1986). Findings are displayed in 118 tables covering forest area, landscape pattern, mast potential, standing dead and cavity trees; and understory woody-stemmed vegetation. Data are presented at county and/or unit and state levels of resolution.

Keywords: Forest habitat survey, inventory, area, landscape pattern, snap, mast, browse. 
Headquarters of the Northeastern Forest Experiment Station is in Broomall, Pennsylvania. Field laboratories are maintained at:

Amherst, Massachusetts, in cooperation with the University of Massachusetts

Berea, Kentucky, in cooperation with Berea College

Burlington, Vermont, in cooperation with the University of Vermont

Delaware, Ohio

Durham, New Hampshire, in cooperation with the University of New Hampshire

Hamden, Connecticut, in cooperation with Yale University

Morgantown, West VIrginia, in cooperation with West Virginia University

Orono, Malne, In cooperation with the University of Maine

Parsons, West Virginia

Princeton, West Virginia

Syracuse, New York, in cooperation with the State University of New York, College of Environmental Sciences and Forestry at Syracuse University

UnlversIty Park, Pennsylvania, in cooperation with The Pennsylvania State University

Warren, Pennsylvania

Persons of any race, color, national origin, sex, age, religion, or with any handicapping condition are welcome to use and enjoy all facilities, programs, and services of the USDA. Discrimination in any form is strictly against agency policy, and should be reported to the Secretary of Agriculture, Washington, DC 20250. 